\title{
Asymmetric Catalysis upon Helically Chiral Loratadine Analogues Unveils Enantiomer-Dependent Antihistamine Activity
}

\author{
Elizabeth A. Stone, Kara J. Cutrona, and Scott J. Miller* \\ Department of Chemistry, Yale University, New Haven, CT 06520, United States \\ *Email:scott.miller@yale.edu
}

\section{Supporting Information}

\section{Table of Contents}

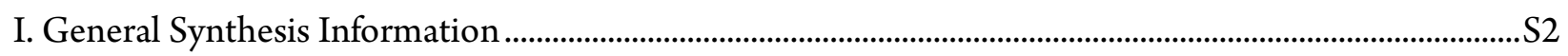

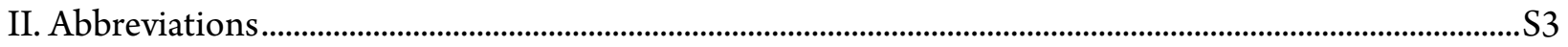

III. Synthesis and Characterization of Peptide Catalysts ..........................................................................................

IV. Synthesis and Characterization of Loratadine Analogs .................................................................................

V. Synthesis and Characterization of Loratadine $\mathrm{N}$-Oxide Analogs ............................................................... S12

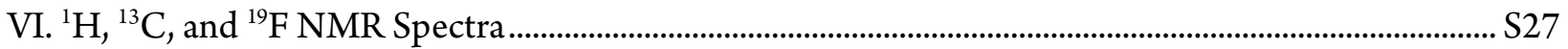

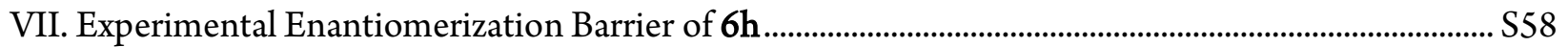

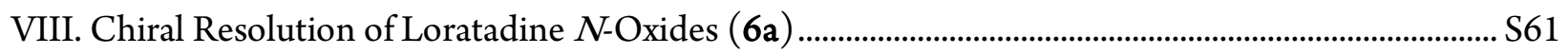

VIIII. Antihistamine Activity Testing of Loratadine Analogs.............................................................................. S63

X. DFT Computational Studies .......................................................................................................................... S65

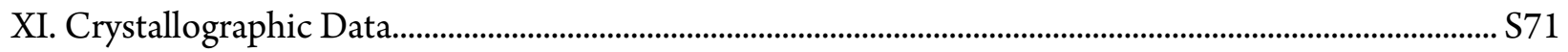

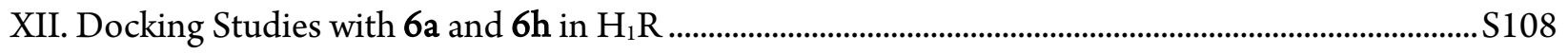

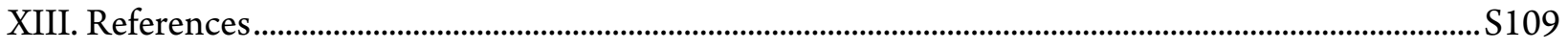




\section{General Synthesis Information}

All reactions were carried out under ambient atmosphere unless otherwise noted. Room temperature (rt) is defined as $21-23{ }^{\circ} \mathrm{C}$. Acetonitrile $(\mathrm{MeCN})$, dichloromethane (DCM), Tetrahydrofuran (THF), $N, N$-dimethylformamide (DMF), and toluene (PhMe) were dried over alumina and dispensed under argon from a Glass Contour Seca Solvent Purification System. All reagents were obtained from commercial sources and used without further purification. $\mathrm{N}, \mathrm{N}$-diisopropyl ethylamine $\left(\mathrm{Pr}_{2} \mathrm{NEt}\right.$ ) was distilled over $\mathrm{CaH}$ under a nitrogen atmosphere prior to use. Deionized water was used for reactions, extraction solutions, and reverse-phase chromatography. All other solvents used for chromatography were HPLC grade.

Infrared spectra were recorded on a Nicolet 6700 ATR/FT-IR spectrometer with select $v_{\max }$ reported in $\mathrm{cm}^{-1}$. High-resolution mass spectrometry (HRMS) was conducted by the Chemical and Biophysical Instrumentation Center in the Chemistry Department at Yale University using a Waters Xevo Q-TOF high-resolution mass spectrometer using electrospray ionization (ESI). For crude data analysis, ultra high-performance liquid chromatography-mass spectrometry (UPLC/MS) was performed with a Waters Acquity UPLC/MS instrument equipped with a reversed-phase BEH C18 column $(1.7 \mathrm{~mm}$ particle size, $2.1 \times 50 \mathrm{~mm})$, a dual atmospheric pressure chemical ionization (API)/electrospray ionization (ESI) mass spectrometry detector, and a photodiode array detector. Analytical thin-layer chromatography (TLC) was performed using 60 Å Silica Gel F 254 pre-coated plates $(0.25 \mathrm{~mm}$ thickness). TLC plates were visualized by irradiation with a UV lamp. Flash chromatography was performed using a Biotage Isolera One purification system equipped with a 10, 25, 50, or $100 \mathrm{~g}$ SNAP Ultra (HP Sphere, $25 \mu \mathrm{m}$ silica) cartridge for normal-phase column chromatography, and 12, 30, 60, or $120 \mathrm{~g}$ SNAP-C18 columns for reversephase column chromatography.

Routine ${ }^{1} \mathrm{H}$ NMR spectra were recorded on Agilent 500 or $600 \mathrm{MHz}$ spectrometers at ambient temperature unless otherwise stated. NMR solvents, chloroform- $d\left(\mathrm{CDCl}_{3}\right)$ and methanol- $d_{4}\left(\mathrm{CD}_{3} \mathrm{OD}\right)$ were purchased from Cambridge Isotope Laboratories and used without further purification. $\mathrm{CDCl}_{3}$ was stored at ambient temperature over 4 Å molecular sieves, and fresh $\mathrm{CD}_{3} \mathrm{OD}$ ampules were used immediately after opening. Spectra were processed using MestReNova 10.0.1 using the automatic phasing and polynomial baseline correction capabilities. Splitting was determined using the automatic multiplet analysis function with intervention as necessary. Spectral data are reported as follows: chemical shift (multiplicity [ $\operatorname{singlet}(\mathrm{s})$, broad singlet $(\mathrm{bs})$, doublet $(\mathrm{d})$, triplet $(\mathrm{t})$, quartet $(\mathrm{q})$, pentet $(\mathrm{p})$, multiplet $(\mathrm{m})$, doublet of doublets $(\mathrm{dd})$, doublet of doublet of doublets (ddd), doublet of triplet of doublets ( $\mathrm{dtd}$ ), doublet of doublet of doublet of doublets (dddd), doublet of triplets (dt), triplet of doublets $(\mathrm{td})$, complex (comp), etc.], coupling constant $(\mathrm{Hz})$, integration). Chemical shifts are reported in ppm $(\delta)$, and coupling constants are reported in $\mathrm{Hz} .{ }^{1} \mathrm{H}$ Resonances are referenced to solvent residual peaks for $\mathrm{CDCl}_{3}$ (7.26 ppm) or $\mathrm{CD}_{3} \mathrm{OD}(3.31 \mathrm{ppm})$. Routine ${ }^{13} \mathrm{C}$ NMR spectra were recorded on Agilent 500 or $600 \mathrm{MHz}$ spectrometers with protons fully decoupled. ${ }^{13} \mathrm{C}$ Resonances are reported in ppm relative to solvent residual peaks for $\mathrm{CDCl}_{3}$ (77.2 $\mathrm{ppm})$ or $\mathrm{CD}_{3} \mathrm{OD}(49.0 \mathrm{ppm}) .{ }^{19} \mathrm{~F}-\mathrm{NMR}$ spectra were recorded on Agilent $500 \mathrm{MHz}$ spectrometers without proton decoupling.

Optical rotations were recorded on an Autopol VI Automatic Polarimeter at the sodium D-line ( $589 \mathrm{~nm})$, unless otherwise indicated, using a Type $40 \mathrm{~T}$ TempTrolTM cell of $0.50 \mathrm{dm}$ path length at $20^{\circ} \mathrm{C}$ and reported as follows: $[\alpha]_{\lambda}^{\text {temp }}$, concentration ( $\mathrm{c}$ in $\mathrm{g} / 100 \mathrm{~mL}$ ), and solvent. Normal-phase high-performance liquid chromatography (HPLC) was performed using an Agilent 1100 series instrument equipped with a diode array detector and columns (chiral supports, $5 \mu \mathrm{m}$ particle size, $4.6 \times 250 \mathrm{~mm}$ ) from Daicel Chemical Industries. 


\section{Abbreviations}

( $\alpha$-Me)Pro: $\alpha$-methyl-L-proline

Acpc: 1-aminocyclopropane carboxylic acid

Boc: tert-butoxycarbonyl

Cbz: carboxybenzyl

DIC: $N, N$-diisopropylcarbodiimide

DIPEA: diisopropyl ethyl amine

DMSO: dimethyl sulfoxide

EDC: $N$-(3-dimethylaminopropyl)- $N$-ethylcarbodiimide hydrochloride

er: enantiomeric ratio

ESI: electrospray ionization

FLIPR: fluorescence imagine plate reader

HATU: $O$-(7-aza-1-benzotriazolyl)- $N, N, N$,' $N$ '-tetramethyluronium hexafluorophosphate

HOBt: 1-hydroxybenzotriazole

HPLC: high-performance liquid chromatography

HRMS: high-resolution mass spectrometry

IR: infrared

$m$-CPBA: meta-chloroperoxybenzoic acid

NMR: nuclear magnetic resonance

$R P$ : reverse-phase

rt or RT: room temperature

sat.: saturated

TLC: thin-layer chromatography

Ts: tosyl

UPLC/MS: ultra high-performance liquid chromatography-mass spectrometry 


\section{Synthesis and Characterization of Peptide Catalysts}

The solution phase synthesis of all peptide catalysts was accomplished using the $N$-tert-butoxycarbonyl (Boc) protecting group strategy. The synthesis and characterization of peptides $\mathbf{P} 1$ and $\mathbf{P} 2$ were previously reported by Miller and co-workers. ${ }^{1}$ The synthesis of peptides P5 and P6 are shown below as examples to depict the general strategy.

(i)

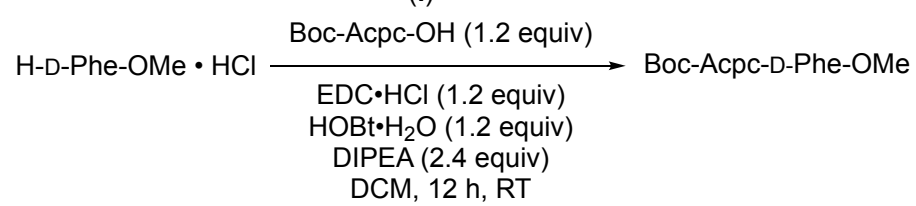

(ii)

$\mathrm{HCl}$ in dioxanes (4 M, 8.0 equiv)

$1 \mathrm{~h}, \mathrm{RT}$ (iii)

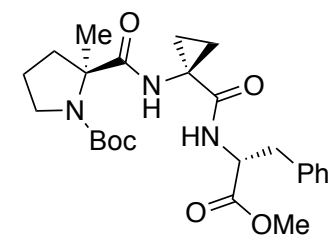

(iv) (4 $\mathrm{M}, 8.0$ equiv) $1 \mathrm{~h}, \mathrm{RT}$

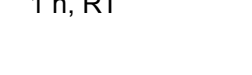
$\mathrm{HCl}$ in dioxanes
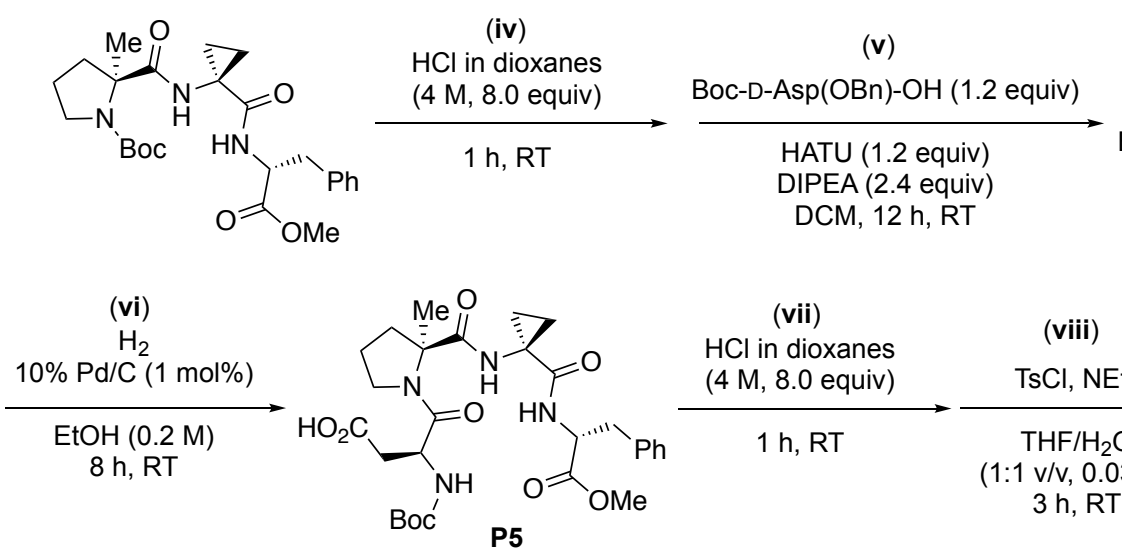

(vii)

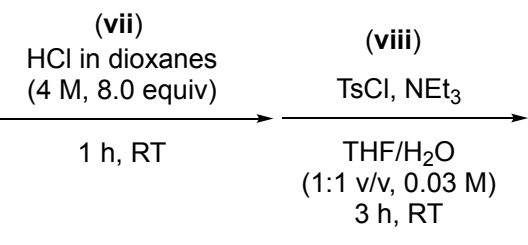

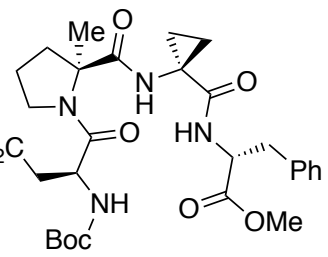
$\mathrm{HOBt} \cdot \mathrm{H}_{2} \mathrm{O}(1.2$ equiv) DIPEA (2.4 equiv) DCM, 12 h, RT

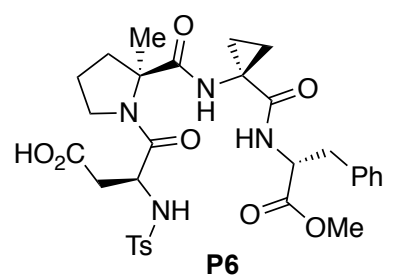

(i) H-D-Phe-OMe-HCl (0.908 g, $5.0 \mathrm{mmol}, 1.0$ equiv), Boc-Acpc-OH (1.207 g, $6.0 \mathrm{mmol}, 1.2$ equiv), EDC.HCl ( $1.150 \mathrm{~g}, 6.0 \mathrm{mmol}, 1.2$ equiv), and $\mathrm{HOBt} \cdot \mathrm{H}_{2} \mathrm{O}(0.919 \mathrm{~g}, 6.0 \mathrm{mmol}, 1.2$ equiv $)$ were dissolved in $\mathrm{DCM}(20.0 \mathrm{~mL}$, $0.25 \mathrm{M})$ followed by addition of DIPEA $(2.1 \mathrm{~mL}, 12.0 \mathrm{mmol}, 2.4$ equiv). The reaction mixture was stirred at room temperature for $12 \mathrm{~h}$, and then the organics were washed with citric acid $(10 \% \mathrm{v} / \mathrm{v})$, sat. $\mathrm{NaHCO}_{3}(a q)$, then brine. The combined organic layer was dried over $\mathrm{Na}_{2} \mathrm{SO}_{4}$, filtered, and concentrated in vacuo. The residue (Boc-Acpc$\mathrm{D}-\mathrm{Phe}-\mathrm{OMe}$ ) was then used directly in the next step without further purification.

(ii) To the peptide residue (Boc-Acpc- D-Phe-OMe), $\mathrm{HCl}(4 \mathrm{~N}$ in dioxane, $10.0 \mathrm{~mL}, 40.0 \mathrm{mmol}$, 8.0 equiv) was added. The reaction was stirred at room temperature for $1 \mathrm{~h}$ followed by evaporation in vacuo to dryness. The deprotected peptide was used directly without further purification.

(iii) To a $50 \mathrm{~mL}$ round bottom flask equipped with a stir bar, the peptide residue (H-Acpc-D-Phe -OMe• $\mathrm{HCl}$ ), Boc- $(\alpha-\mathrm{Me})$ Pro-OH (1.146 g, $5.0 \mathrm{mmol}, 1.0$ equiv), EDC. $\mathrm{HCl}\left(1.150,6.0 \mathrm{mmol}, 1.2\right.$ equiv), and $\mathrm{HOBt}_{2} \mathrm{H}_{2} \mathrm{O}$ $(0.919 \mathrm{~g}, 6.0 \mathrm{mmol}, 1.2$ equiv $)$ were dissolved in DCM $(20.0 \mathrm{~mL}, 0.25 \mathrm{M})$ followed by DIPEA $(2.1 \mathrm{~mL}, 12.0 \mathrm{mmol}$, 2.4 equiv). The reaction mixture was stirred at room temperature for $12 \mathrm{~h}$, and the volatiles were removed under reduced pressure then the organics were washed with citric acid $(10 \% \mathrm{v} / \mathrm{v})$, sat. $\mathrm{NaHCO}_{3}(a q)$, then brine. The combined organic layer was dried over $\mathrm{Na}_{2} \mathrm{SO}_{4}$, filtered, and concentrated in vacuo. The crude material was purified subjected to reverse-phase column chromatography (SNAP Ultra C18 120g, gradient $=30-100 \% \mathrm{MeOH} / \mathrm{H}_{2} \mathrm{O}$ for $10 \mathrm{CV}$ ). The partially pure material was subjected to a second reverse-phase column (SNAP Ultra C18 120g, gradient $=0-100 \% \mathrm{MeCN} / \mathrm{H}_{2} \mathrm{O}$ for $\left.10 \mathrm{CV}\right)$ to afford Boc- $(\alpha-\mathrm{Me})$ Pro-Acpc-D-Phe-OMe as a white solid (0.456 g, $0.96 \mathrm{mmol}, 19 \%$ yield over 3 steps). 
(iv) To the peptide residue (Boc-( $\alpha$-Me)Pro-Acpc-D-Phe-OMe, $0.1856 \mathrm{~g}, 0.39 \mathrm{mmol}, 1.0$ equiv), $\mathrm{HCl}(4 \mathrm{~N}$ in dioxane, $0.78 \mathrm{~mL}, 3.14 \mathrm{mmol}, 8.0$ equiv) was added. The reaction was stirred at room temperature for $1 \mathrm{~h}$ followed by evaporation in vacuo to dryness. The deprotected peptide was used directly without further purification.

(v) To a 5-dram vial equipped with a stir bar, the peptide residue (H- $(\alpha-\mathrm{Me})$ Pro-Acpc-D-Phe-OMe $\cdot \mathrm{HCl})$, Boc-D$\operatorname{Asp}(\mathrm{OBn})-\mathrm{OH}(0.1519 \mathrm{~g}, 0.47 \mathrm{mmol}, 1.2$ equiv), and HATU ( $0.1787 \mathrm{~g}, 0.47 \mathrm{mmol}, 1.2$ equiv) were dissolved in $\operatorname{DCM}(2 \mathrm{~mL}, 0.2 \mathrm{M})$, followed by addition of DIPEA $(0.16 \mathrm{~mL}, 0.94 \mathrm{mmol}, 2.4$ equiv). The reaction mixture was stirred at room temperature for $12 \mathrm{~h}$, then the organics were washed with citric acid (10\% v/v), sat. $\mathrm{NaHCO}_{3}(a q)$, then brine. The combined organic layer was dried over $\mathrm{Na}_{2} \mathrm{SO}_{4}$, filtered, and concentrated in vacuo. The crude material was purified subjected to reverse-phase column chromatography (SNAP Ultra C18 60g, gradient $=0$ $100 \% \mathrm{MeCN} / \mathrm{H}_{2} \mathrm{O}$ for $\left.12 \mathrm{CV}\right)$ to afford peptide Boc-Asp(OBn-( $\alpha$-Me)Pro-Acpc-D-Phe-OMe (0.2285 g, 0.34 mmol, $87 \%$ yield).

(vii) To an oven dried $10 \mathrm{~mL}$ round bottom flask equipped with a stir bar, peptide Boc-Asp(OBn)-Pro-Acpc-DPhe-OMe (0.2285 g, $0.34 \mathrm{mmol}, 1.00$ equiv) was dissolved in $\mathrm{EtOH}(0.7 \mathrm{~mL}, 0.5 \mathrm{M})$ followed by $10 \% \mathrm{Pd} / \mathrm{C}(23.0$ $\mathrm{mg}, 0.034 \mathrm{mmol}, 0.01$ equiv). The reaction flask was evacuated and refilled with $\mathrm{H}_{2}(3 \mathrm{x})$, then reaction was stirred at room temperature for $20 \mathrm{~h}$. The reaction was filtered through a pad of celite, washing with DCM/EtOH. The filtrate was evaporated in vacuo, then subjected to reverse-phase column chromatography (SNAP Ultra C18 30g, gradient $=0-82 \% \mathrm{MeOH} / \mathrm{H}_{2} \mathrm{O}$ with $0.1 \%$ formic acid additive for $8 \mathrm{CV}$, then $82 \% \mathrm{MeOH} / \mathrm{H}_{2} \mathrm{O}$ with $0.1 \%$ formic acid additive for $2 \mathrm{CV}$, then $82-100 \% \mathrm{MeOH} / \mathrm{H}_{2} \mathrm{O}$ with $0.1 \%$ formic acid additive for $\left.2 \mathrm{CV}\right)$ to afford P5 $(0.1981$ g, $0.34 \mathrm{mmol}$, quant. yield) as a white foamy solid. ${ }^{1} \mathrm{H} \mathrm{NMR}\left(600 \mathrm{MHz}, \mathrm{CDCl}_{3}\right) \delta 7.78(\mathrm{~s}, 1 \mathrm{H}), 7.34-7.09(\mathrm{~m}$,

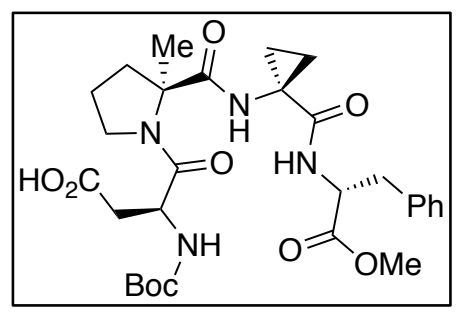
$4 \mathrm{H}), 5.17(\mathrm{~d}, J=7.7 \mathrm{~Hz}, 1 \mathrm{H}), 4.70(\mathrm{~s}, 1 \mathrm{H}), 4.55(\mathrm{~s}, 1 \mathrm{H}), 3.98(\mathrm{~s}, 1 \mathrm{H}), 3.81(\mathrm{~s}$, $1 \mathrm{H}), 3.57(\mathrm{~s}, 3 \mathrm{H}), 3.18-3.12(\mathrm{~m}, 1 \mathrm{H}), 3.10-3.03(\mathrm{~m}, 2 \mathrm{H}), 2.67(\mathrm{~d}, J=15.8 \mathrm{~Hz}$, $1 \mathrm{H}), 2.11-1.78(\mathrm{~m}, 4 \mathrm{H}), 1.59-1.49(\mathrm{~m}, 4 \mathrm{H}), 1.42(\mathrm{~s}, 9 \mathrm{H}), 0.92(\mathrm{~s}, 1 \mathrm{H}), 0.86(\mathrm{~s}$, 1H) [Note: $\mathrm{NH}$ and $\mathrm{CO}_{2} \mathrm{H}$ resonances were not observed].${ }^{13} \mathrm{C}$ NMR (151 $\left.\mathrm{MHz}, \mathrm{CDCl}_{3}\right) \delta 174.1,173.8,173.7,173.0,170.5,155.1,136.5,129.4,128.4$, 126.9, 80.6, 67.9, 55.3, 52.4, 48.9, 48.6, 39.1, 37.6, 36.9, 34.4, 28.3, 23.6, 20.5, 17.5. HRMS (ESI) $\mathrm{m} / \mathrm{z}:[\mathrm{M}+\mathrm{H}]^{+}$calcd for $\mathrm{C}_{29} \mathrm{H}_{40} \mathrm{~N}_{4} \mathrm{O}_{9} 589.2868$, found 589.2896.

(vii) To a 5-dram vial equipped with a stir bar, P5 (40.0 mg, $0.067 \mathrm{mmol}, 1.00$ equiv) was added and dissolved in $\mathrm{HCl}$ ( $4 \mathrm{~N}$ in 1,4-dioxanes, $0.13 \mathrm{~mL}, 0.54 \mathrm{mmol}, 8.0$ equiv) while stirring at room temperature. After $1 \mathrm{~h}$, the solution was concentrated in vacuo and used without further purification.

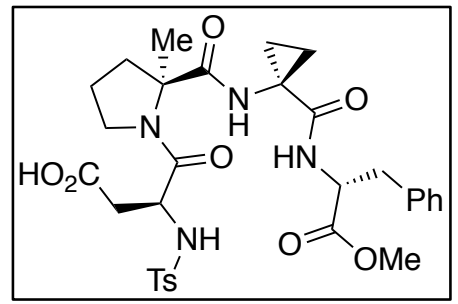

(viii) The dried, deprotected peptide (Asp(OBn)-Pro-Acpc-D-Phe-OMe. $\mathrm{HCl}$ ) was dissolved in $\mathrm{THF} / \mathrm{H}_{2} \mathrm{O}(1: 1 \mathrm{v} / \mathrm{v}, 0.03 \mathrm{M})$ at $0{ }^{\circ} \mathrm{C}$ while stirring. To the solution, $\mathrm{TsCl}$ ( $14.1 \mathrm{mg}, 0.074 \mathrm{mmol}, 1.1$ equiv) was added followed by dropwise addition of triethyl amine $(0.04 \mathrm{~mL}, 0.27 \mathrm{mmol}, 4.0$ equiv). The reaction was allowed to warm to room temperature while stirring for $3 \mathrm{~h}$. The crude material was purified using reverse-phase column chromatography (SNAP Ultra C18 30g, gradient $=0-80 \% \mathrm{MeOH} / \mathrm{H}_{2} \mathrm{O}$ with $0.1 \%$ formic acid additive for $8 \mathrm{CV}$, then $80 \%$ $\mathrm{MeOH} / \mathrm{H}_{2} \mathrm{O}$ with $0.1 \%$ formic acid additive for $2 \mathrm{CV}$, then $80-100 \% \mathrm{MeOH} / \mathrm{H}_{2} \mathrm{O}$ with $0.1 \%$ formic acid additive for $2 \mathrm{CV}$ ) to afford P6 as a white solid $\left(26.3 \mathrm{mg}, 0.041 \mathrm{mmol}, 63 \%\right.$ yield over two steps). ${ }^{1} \mathrm{H}$ NMR (600 MHz, $\left.\mathrm{CDCl}_{3}\right) \delta 7.70(\mathrm{~s}, 2 \mathrm{H}), 7.58(\mathrm{~s}, 1 \mathrm{H}), 7.34-7.15(\mathrm{~m}, 5 \mathrm{H}), 6.97(\mathrm{~s}, 1 \mathrm{H}), 5.53(\mathrm{~d}, J=9.0 \mathrm{~Hz}, 1 \mathrm{H}), 4.50(\mathrm{~s}, 1 \mathrm{H}), 4.42$ (s, 1H), $3.70(\mathrm{~s}, 2 \mathrm{H}), 3.59(\mathrm{~s}, 3 \mathrm{H}), 3.14-2.88(\mathrm{~m}, 3 \mathrm{H}), 2.57(\mathrm{~d}, J=15.5 \mathrm{~Hz}, 1 \mathrm{H}), 2.41(\mathrm{~s}, 3 \mathrm{H}), 1.89(\mathrm{~s}, 3 \mathrm{H}), 1.50$ $(\mathrm{s}, 1 \mathrm{H}), 1.37(\mathrm{~s}, 1 \mathrm{H}), 1.28(\mathrm{~s}, 3 \mathrm{H}), 0.89(\mathrm{~s}, 1 \mathrm{H}), 0.82(\mathrm{~s}, 1 \mathrm{H})$ [Note: $\mathrm{NH}$ and $\mathrm{CO}_{2} H$ resonances were not observed]. ${ }^{13} \mathrm{C}$ NMR $\left(151 \mathrm{MHz}, \mathrm{CDCl}_{3}\right) \delta 174.6,173.5,173.2,172.9,169.1,144.3,137.4,136.4,130.1,129.4,128.6,127.1$, 127.1, 68.2, 55.5, 52.7, 51.2, 48.4, 38.9, 37.9, 37.5, 23.7, 21.7, 20.1, 17.6, 0.1. HRMS (ESI) $\mathrm{m} / \mathrm{z}:[\mathrm{M}+\mathrm{H}]^{+}$calcd for $\mathrm{C}_{31} \mathrm{H}_{38} \mathrm{~N}_{4} \mathrm{O}_{9} \mathrm{~S}$ 643.2432, found 643.2438. 


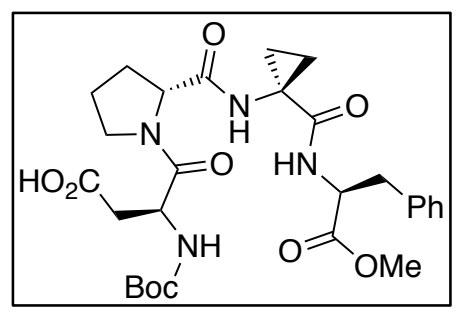

P3: Using D-Pro-Acpc-Phe-OMe ( $0.230 \mathrm{~g}, 0.50 \mathrm{mmol}, 1.00$ equiv) (synthesis described by Miller and co-workers on route to $\mathbf{P 2})^{1}$, with steps (iv-vi), $\mathbf{P} 3$ was produced as a white solid ( $0.123 \mathrm{~g}, 0.21 \mathrm{mmol}, 86 \%$ yield over 3 steps $).{ }^{1} \mathrm{H}$ NMR $(600$ $\left.\mathrm{MHz}, \mathrm{CDCl}_{3}\right) \delta 7.61(\mathrm{~d}, J=8.0 \mathrm{~Hz}, 1 \mathrm{H}), 7.31-7.23(\mathrm{~m}, 2 \mathrm{H}), 7.23-7.15(\mathrm{~m}$, $4 \mathrm{H}), 6.13(\mathrm{~d}, J=8.4 \mathrm{~Hz}, 1 \mathrm{H}), 4.79(\mathrm{q}, J=7.5 \mathrm{~Hz}, 1 \mathrm{H}), 4.73(\mathrm{td}, J=8.3,5.1 \mathrm{~Hz}$, $1 \mathrm{H}), 4.27(\mathrm{t}, J=7.1 \mathrm{~Hz}, 1 \mathrm{H}), 3.74(\mathrm{t}, J=6.6 \mathrm{~Hz}, 2 \mathrm{H}), 3.59(\mathrm{~s}, 3 \mathrm{H}), 3.13(\mathrm{dd}, J=$ $13.7,7.4 \mathrm{~Hz}, 1 \mathrm{H}), 3.07(\mathrm{dd}, J=13.7,7.4 \mathrm{~Hz}, 1 \mathrm{H}), 2.90(\mathrm{dd}, J=16.7,8.4 \mathrm{~Hz}, 1 \mathrm{H})$, $2.72(\mathrm{dd}, J=16.7,4.9 \mathrm{~Hz}, 1 \mathrm{H}), 2.16(\mathrm{dq}, J=12.0,6.2,5.7 \mathrm{~Hz}, 1 \mathrm{H}), 2.05(\mathrm{dt}, J=12.0,5.9 \mathrm{~Hz}, 1 \mathrm{H}), 1.92(\mathrm{ddq}, J=$ 26.6, 14.6, 7.2 Hz, 2H), $1.52-1.47(\mathrm{~m}, 2 \mathrm{H}), 1.43(\mathrm{~s}, 9 \mathrm{H}), 0.95(\mathrm{qd}, J=9.8,3.0 \mathrm{~Hz}, 2 \mathrm{H})$ [Note: the $\mathrm{CO}_{2} H$ resonance was not observed]. ${ }^{13} \mathrm{C} \mathrm{NMR}\left(151 \mathrm{MHz}, \mathrm{CDCl}_{3}\right) \delta 174.1,173.5,172.7,171.5,171.3,155.9,136.6,129.4,128.5$, 126.9, 80.8, 61.8, 54.3, 52.4, 49.6, 48.0, 38.2, 36.7, 34.6, 29.1, 25.5, 17.5, 17.2. HRMS (ESI) $\mathrm{m} / z$ : $[\mathrm{M}+\mathrm{H}]^{+}$calcd for $\mathrm{C}_{28} \mathrm{H}_{38} \mathrm{~N}_{4} \mathrm{O}_{9}$ 575.2712, 575.2732; (ESI) $\mathrm{m} / \mathrm{z}:[\mathrm{M}+\mathrm{Na}]^{+}$calcd for $\mathrm{C}_{28} \mathrm{H}_{38} \mathrm{~N}_{4} \mathrm{O}_{9}$ 597.2531, 597.2559.

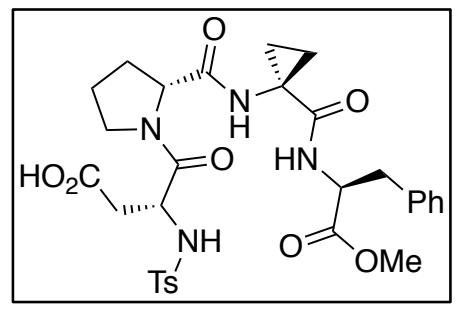

P4: Using P2 ${ }^{1}$ ( $0.181 \mathrm{~g}, 0.31 \mathrm{mmol}, 1.0$ equiv) with steps (vii) and (viii), the crude material was purified using reverse-phase column chromatography (SNAP Ultra $\mathrm{C} 1830 \mathrm{~g}$, gradient $=20-100 \% \mathrm{MeCN} / \mathrm{H}_{2} \mathrm{O}$ over $15 \mathrm{CV}$ ) to afford $\mathbf{P 4}$ as a white solid $\left(0.161 \mathrm{~g}, 0.26 \mathrm{mmol}, 83 \%\right.$ yield over two steps). ${ }^{1} \mathrm{H}$ NMR $(600 \mathrm{MHz}$, $\left.\mathrm{CDCl}_{3}\right) \delta 7.70(\mathrm{~s}, 2 \mathrm{H}), 7.37-7.18(\mathrm{~m}, 5 \mathrm{H}), 7.13(\mathrm{~s}, 2 \mathrm{H}), 6.01(\mathrm{~d}, J=9.0 \mathrm{~Hz}, 1 \mathrm{H})$, $4.58(\mathrm{~s}, 1 \mathrm{H}), 4.41(\mathrm{~s}, 1 \mathrm{H}), 4.07(\mathrm{~s}, 1 \mathrm{H}), 3.64(\mathrm{~s}, 1 \mathrm{H}), 3.59(\mathrm{~s}, 2 \mathrm{H}), 3.53(\mathrm{~s}, 1 \mathrm{H})$, $3.11-2.96(\mathrm{~m}, 3 \mathrm{H}), 2.55(\mathrm{~d}, J=16.6 \mathrm{~Hz}, 1 \mathrm{H}), 2.42(\mathrm{~s}, 3 \mathrm{H}), 2.08-1.80(\mathrm{~m}, 2 \mathrm{H})$, $1.80-1.67(\mathrm{~m}, 2 \mathrm{H}), 1.43(\mathrm{~s}, 1 \mathrm{H}), 1.38(\mathrm{~s}, 1 \mathrm{H}), 0.91(\mathrm{~s}, 1 \mathrm{H}), 0.84(\mathrm{~s}, 1 \mathrm{H})$ [Note: $\mathrm{N} H$ and $\mathrm{CO}_{2} H$ resonances were not observed]. ${ }^{13} \mathrm{C}$ NMR $\left(151 \mathrm{MHz}, \mathrm{CDCl}_{3}\right) \delta 173.5,172.9,172.4,172.2,170.3,144.2,137.5,136.2,130.0,129.4$, 128.6 , 127.1, 127.0, 61.5, 54.8, 52.5, 50.5, 47.5, 37.7, 37.3, 34.3, 29.3, 24.5, 21.7, 17.3, 17.1, 0.1. HRMS (ESI) $\mathrm{m} / \mathrm{z}$ : $[\mathrm{M}+\mathrm{H}]^{+}$calcd for $\mathrm{C}_{30} \mathrm{H}_{36} \mathrm{~N}_{4} \mathrm{O}_{9} \mathrm{~S} 629.2276$, found 629.2302. 


\section{Synthesis and Characterization of Loratadine Analogs}<smiles>Clc1ccc2c(c1)CCc1cccnc1C2=C1CCNCC1</smiles><smiles>[R]NC(=O)N1CCC(=C2c3ccc(Cl)cc3CCc3cccnc32)CC1</smiles>

General Procedure: To a 5-dram vial equipped with a stir bar, desloratadine ( $0.311 \mathrm{~g}, 1.00 \mathrm{mmol}, 1.00$ equiv $)$ was added and dissolved in THF $(2.5 \mathrm{~mL})$. Then, the isocyanate $(1.30 \mathrm{mmol}, 1.30$ equiv) was added and the reaction was allowed to stir at $\mathrm{rt}$ for $3 \mathrm{~h}$ minimum. The cloudy solution was concentrated in vacuo and purified using flash chromatography to yield a solid.

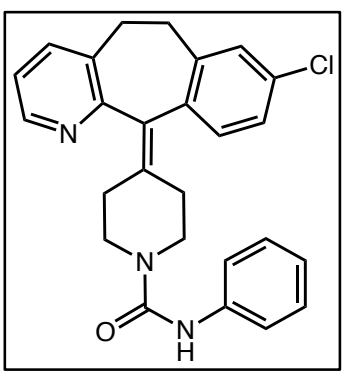

\section{4-(8-Chloro-5,6-dihydro-11 $H$-benzo[5,6]cyclohepta[1,2-b]pyridin-11-ylidene) $-N$-phenylpiperidine-1-carboxamide (1b)}

Using the general procedure with phenyl isocyanate $(0.14 \mathrm{~mL}, 1.30 \mathrm{mmol}, 1.30$ equiv), the crude product was purified using normal-phase column chromatography (SNAP Ultra $100 \mathrm{~g}$, gradient $=0-15 \% \mathrm{DCM} / \mathrm{MeOH}$ for $10 \mathrm{CV})$ to afford a white solid $(0.43 \mathrm{~g}, 0.99$ mmol, 99\% yield). TLC: $\mathrm{R}_{\mathrm{f}}=0.43$ (80\% EtOAc/Hex). IR (FT-ATR, neat): 1666, 1535, $1440,1367,1322,1210,756 \mathrm{~cm}^{-1} .{ }^{1} \mathrm{H}$ NMR $\left(600 \mathrm{MHz}, \mathrm{CD}_{3} \mathrm{OD}\right) \delta 8.34$ (dd, $J=4.9,1.6$ $\mathrm{Hz}, 1 \mathrm{H}), 7.67(\mathrm{dd}, J=7.8,1.5 \mathrm{~Hz}, 1 \mathrm{H}), 7.33(\mathrm{dd}, J=8.6,1.2 \mathrm{~Hz}, 2 \mathrm{H}), 7.29-7.22(\mathrm{~m}$, $4 \mathrm{H}), 7.19(\mathrm{dd}, J=8.2,2.2 \mathrm{~Hz}, 1 \mathrm{H}), 7.15(\mathrm{~d}, J=8.2 \mathrm{~Hz}, 1 \mathrm{H}), 7.00(\mathrm{tt}, J=7.4,1.1 \mathrm{~Hz}, 1 \mathrm{H})$, $3.83(\mathrm{dq}, J=13.3,4.4 \mathrm{~Hz}, 2 \mathrm{H}), 3.47-3.37(\mathrm{~m}, 2 \mathrm{H}), 3.35-3.24(\mathrm{~m}, 2 \mathrm{H}), 2.93-2.79(\mathrm{~m}, 2 \mathrm{H}), 2.48$ (ddt, $J=13.6$, $8.8,4.2 \mathrm{~Hz}, 2 \mathrm{H}), 2.38$ (ddd, $J=14.2,5.8,3.8 \mathrm{~Hz}, 1 \mathrm{H}), 2.25$ (ddd, $J=14.4,5.9,3.8 \mathrm{~Hz}, 1 \mathrm{H}) .{ }^{13} \mathrm{C}$ NMR $(151 \mathrm{MHz}$, $\left.\mathrm{CD}_{3} \mathrm{OD}\right) \delta 158.4,157.8,147.1,141.2,141.0,139.7,139.0,138.5,135.9,134.8,134.3,131.8,130.4,129.6,127.1$, 124.2, 124.1, 122.2, 46.0, 45.9, 32.6, 32.0, 31.8, 31.7. HRMS (ESI) $\mathrm{m} / z:[\mathrm{M}+\mathrm{H}]^{+}$calcd for $\mathrm{C}_{26} \mathrm{H}_{25} \mathrm{ClN}_{3} \mathrm{O}^{+}$ 430.1681 , found 430.1677 .

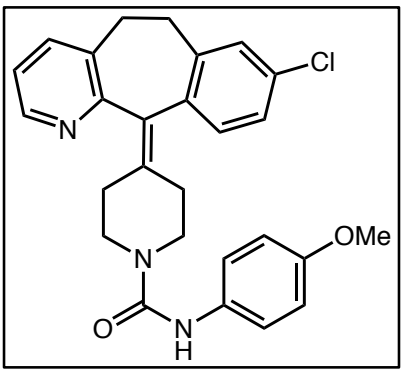

4-(8-Chloro-5,6-dihydro-11 H-benzo[5,6]cyclohepta[1,2-b]pyridin-11-ylidene) - $N$-(4-methoxyphenyl)-piperidine-1-carboxamide (1c)

Using the general procedure with 4-methoxyphenyl isocyanate $(0.11 \mathrm{~mL}, 0.83 \mathrm{mmol}$, 1.30 equiv), the crude product was purified using reverse-phase column chromatography (SNAP Ultra C18 30g, gradient $=40-100 \% \mathrm{MeCN} / \mathrm{H}_{2} \mathrm{O}$ for $8 \mathrm{CV}$ ) to afford a white solid (0.283 g, $0.62 \mathrm{mmol}, 96 \%$ yield). TLC: $\mathrm{R}_{\mathrm{f}}=0.30$ (80\% EtOAc/Hex). IR (FT-ATR, neat): $1627,1510,1436,1414,1240,990,823 \mathrm{~cm}^{-1} .{ }^{1} \mathrm{H}$ NMR $(600 \mathrm{MHz}$, $\left.\mathrm{CD}_{3} \mathrm{OD}\right) \delta 8.33(\mathrm{dd}, J=4.9,1.6 \mathrm{~Hz}, 1 \mathrm{H}), 7.66(\mathrm{dd}, J=7.7,1.6 \mathrm{~Hz}, 1 \mathrm{H}), 7.26(\mathrm{dd}, J=$ 7.7, $4.9 \mathrm{~Hz}, 1 \mathrm{H}), 7.23(\mathrm{~d}, J=2.0 \mathrm{~Hz}, 1 \mathrm{H}), 7.23-7.19(\mathrm{~m}, 2 \mathrm{H}), 7.18(\mathrm{dd}, J=8.2,2.1 \mathrm{~Hz}, 1 \mathrm{H}), 7.14(\mathrm{~d}, J=8.2 \mathrm{~Hz}$, $1 \mathrm{H}), 6.85-6.80(\mathrm{~m}, 2 \mathrm{H}), 3.81(\mathrm{dq}, J=13.5,4.6 \mathrm{~Hz}, 2 \mathrm{H}), 3.75(\mathrm{~s}, 3 \mathrm{H}), 3.47-3.36(\mathrm{~m}, 2 \mathrm{H}), 3.26(\mathrm{dtd}, J=13.0$, 9.1, $3.8 \mathrm{~Hz}, 2 \mathrm{H}), 2.92-2.80(\mathrm{~m}, 2 \mathrm{H}), 2.51-2.42(\mathrm{~m}, 2 \mathrm{H}), 2.40-2.33(\mathrm{~m}, 1 \mathrm{H}), 2.28-2.20(\mathrm{~m}, 1 \mathrm{H}) .{ }^{13} \mathrm{C} \mathrm{NMR}$ $\left(151 \mathrm{MHz}, \mathrm{CD}_{3} \mathrm{OD}\right) \delta 158.4,158.2,157.5,147.1,141.2,139.7,139.0,138.5,135.9,134.7,134.2,133.7,131.8$, 130.4, 127.1, 124.6, 124.2, 114.8, 55.8, 45.9, 45.8, 32.6, 32.0, 31.8, 31.7. HRMS (ESI) $\mathrm{m} / z$ : $[\mathrm{M}+\mathrm{H}]^{+}$calcd for $\mathrm{C}_{27} \mathrm{H}_{27} \mathrm{ClN}_{3} \mathrm{O}_{2}{ }^{+} 460.1786$, found 460.1783 . 


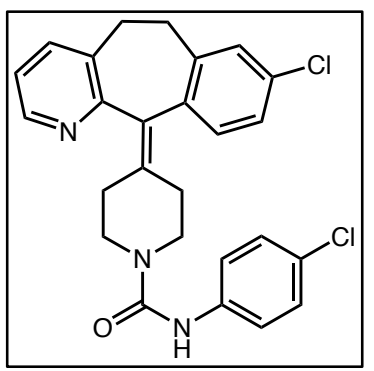

4-(8-Chloro-5,6-dihydro-11 H-benzo[5,6]cyclohepta[1,2-b]pyridin-11-ylidene)$\mathrm{N}$-(4-chlorophenyl)-piperidine-1-carboxamide (1d)

Using the general procedure with 4-chlorophenyl isocyanate $(0.11 \mathrm{~mL}, 0.83 \mathrm{mmol}, 1.30$ equiv), the crude product was purified using reverse-phase column chromatography (SNAP Ultra $\mathrm{C} 1860 \mathrm{~g}$, gradient $=30-100 \% \mathrm{MeCN} / \mathrm{H}_{2} \mathrm{O}$ for $8 \mathrm{CV}$, then $100 \% \mathrm{MeCN}$ for $3 \mathrm{CV}$ ) to afford a white solid $(0.263 \mathrm{~g}, 0.57 \mathrm{mmol}, 88 \%$ yield $)$. TLC: $\mathrm{R}_{\mathrm{f}}=0.48(80 \%$ EtOAc/Hex). IR (FT-ATR, neat): 1634, 1592, 1523, 1492, 1417, 1239, 993, $823 \mathrm{~cm}^{-1}$. ${ }^{1} \mathrm{H}$ NMR $\left(600 \mathrm{MHz}, \mathrm{CD}_{3} \mathrm{OD}\right) \delta 8.32(\mathrm{dd}, J=4.9,1.3 \mathrm{~Hz}, 1 \mathrm{H}), 7.65(\mathrm{dd}, J=7.9,1.0 \mathrm{~Hz}$, $1 \mathrm{H}), 7.37-7.31(\mathrm{~m}, 2 \mathrm{H}), 7.25(\mathrm{dd}, J=7.7,4.9 \mathrm{~Hz}, 1 \mathrm{H}), 7.23-7.22(\mathrm{~m}, 2 \mathrm{H}), 7.22-7.20(\mathrm{~m}, 1 \mathrm{H}), 7.17(\mathrm{dd}, J=$ $8.2,2.1 \mathrm{~Hz}, 1 \mathrm{H}), 7.13(\mathrm{~d}, J=8.2 \mathrm{~Hz}, 1 \mathrm{H}), 3.81(\mathrm{dq}, J=10.5,5.0 \mathrm{~Hz}, 2 \mathrm{H}), 3.46-3.35(\mathrm{~m}, 2 \mathrm{H}), 3.33-3.22(\mathrm{~m}, 2 \mathrm{H})$, $2.91-2.79(\mathrm{~m}, 2 \mathrm{H}), 2.46(\mathrm{ddd}, J=13.6,9.2,4.3 \mathrm{~Hz}, 2 \mathrm{H}), 2.39-2.33(\mathrm{~m}, 1 \mathrm{H}), 2.24(\mathrm{ddd}, J=14.2,5.6,3.9 \mathrm{~Hz}$, $1 \mathrm{H}) .{ }^{13} \mathrm{C}$ NMR $\left(151 \mathrm{MHz}, \mathrm{CD}_{3} \mathrm{OD}\right) \delta 158.4,157.4,147.1,141.2,140.0,139.7,138.8,138.5,135.9,134.8,134.3$, $131.7,130.4,129.5,128.8,127.1,124.2,123.3,46.0,45.8,32.6,32.0,31.8$, 31.6. HRMS (ESI) $\mathrm{m} / z:[\mathrm{M}+\mathrm{H}]^{+}$calcd for $\mathrm{C}_{26} \mathrm{H}_{24} \mathrm{Cl}_{2} \mathrm{~N}_{3} \mathrm{O}^{+} 464.1291$, found 464.1292 .

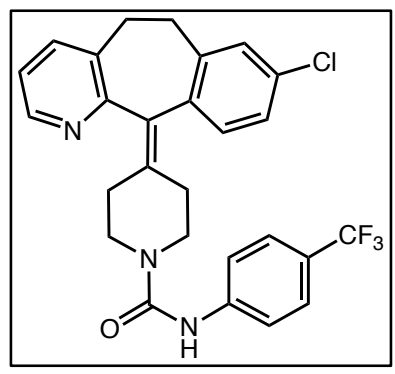

4-(8-Chloro-5,6-dihydro-11 $H$-benzo[5,6]cyclohepta[1,2-b]pyridin-11-ylidene) - $N$-(4-trifluoromethylhenyl)-piperidine-1-carboxamide (1e)

The preparation and characterization of this loratadine analog was previously described by Miller and co-workers. ${ }^{1}$

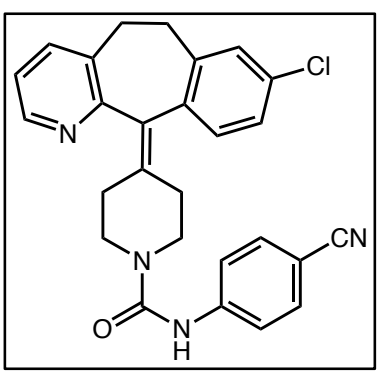

4-(8-Chloro-5,6-dihydro-11 H-benzo[5,6]cyclohepta[1,2-b]pyridin-11-ylidene)$\mathrm{N}$-(4-cyanophenyl)-piperidine-1-carboxamide (1f)

Using the general procedure with 4-cyanophenyl isocyanate $(0.120 \mathrm{~g}, 0.83 \mathrm{mmol}, 1.30$ equiv), the crude product was purified using reverse-phase column chromatography (SNAP Ultra $\mathrm{C} 1830 \mathrm{~g}$, gradient $=40-100 \% \mathrm{MeCN} / \mathrm{H}_{2} \mathrm{O}$ for $8 \mathrm{CV}$ ) to afford a white solid (0.196 g, $0.43 \mathrm{mmol}, 67 \%$ yield). TLC: $\mathrm{R}_{\mathrm{f}}=0.36$ (80\% EtOAc/Hex). IR (FTATR, neat): 2220, 1641, 1589, 1511, 1436, 1414, 1310, 1174, 990, $827 \mathrm{~cm}^{-1} .{ }^{1} \mathrm{H}$ NMR $\left(500 \mathrm{MHz}, \mathrm{CD}_{3} \mathrm{OD}\right) \delta 8.34(\mathrm{dd}, J=4.9,1.2 \mathrm{~Hz}, 1 \mathrm{H}), 7.70-7.64(\mathrm{~m}, 1 \mathrm{H}), 7.65(\mathrm{~s}$, $1 \mathrm{H}), 7.61-7.55(\mathrm{~m}, 4 \mathrm{H}), 7.27(\mathrm{dd}, J=7.7,4.9 \mathrm{~Hz}, 1 \mathrm{H}), 7.24(\mathrm{~d}, J=1.8 \mathrm{~Hz}, 1 \mathrm{H}), 7.19(\mathrm{dd}, J=8.2,2.0 \mathrm{~Hz}, 1 \mathrm{H})$, $7.14(\mathrm{~d}, J=8.2 \mathrm{~Hz}, 1 \mathrm{H}), 3.84(\mathrm{dq}, J=13.0,4.2 \mathrm{~Hz}, 2 \mathrm{H}), 3.42(\mathrm{dtd}, J=13.8,9.6,5.9 \mathrm{~Hz}, 2 \mathrm{H}), 3.36-3.25(\mathrm{~m}, 2 \mathrm{H})$, $2.95-2.80(\mathrm{~m}, 2 \mathrm{H}), 2.48(\mathrm{ddt}, J=13.5,8.6,4.1 \mathrm{~Hz}, 2 \mathrm{H}), 2.42-2.35(\mathrm{~m}, 1 \mathrm{H}), 2.29-2.22(\mathrm{~m}, 1 \mathrm{H}) .{ }^{13} \mathrm{C}$ NMR $(151$ $\left.\mathrm{MHz}, \mathrm{CD}_{3} \mathrm{OD}\right) \delta 158.3,156.7,147.1,146.2,141.2,139.7,138.7,138.5,135.9,134.9,134.3,133.9,131.7,130.4$, 127.1, 124.2, 120.9, 120.2, 105.8, 49.0, 46.1, 46.0, 32.6, 32.0, 31.7, 31.6. HRMS (ESI) $\mathrm{m} / z:[\mathrm{M}+\mathrm{H}]^{+}$calcd for $\mathrm{C}_{27} \mathrm{H}_{24} \mathrm{ClN}_{4} \mathrm{O}^{+} 455.1633$, found 455.1643 .

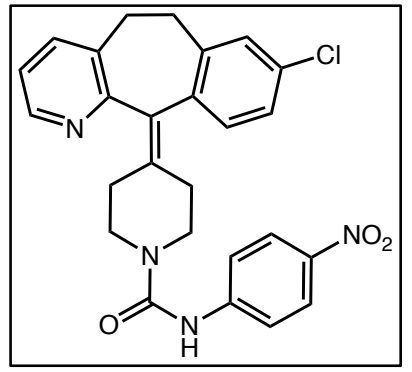

4-(8-Chloro-5,6-dihydro-11 H-benzo[5,6]cyclohepta[1,2-b]pyridin-11-ylidene) - $N$-(4-nitrophenyl)-piperidine-1-carboxamide (1g)

Using the general procedure with 4-nitrophenyl isocyanate $(0.107 \mathrm{~g}, 0.65 \mathrm{mmol}, 1.30$ equiv), the crude product was purified using normal-phase column chromatography (SNAP Ultra $25 \mathrm{~g}$, gradient $=10-100 \% \mathrm{EtOAc} / \mathrm{Hex}$ for $5 \mathrm{CV}$, then $100 \%$ EtOAc for $10 \mathrm{CV})$ to afford a pale-yellow solid $(0.237 \mathrm{~g}, 0.50 \mathrm{mmol}$, quant. yield $)$. TLC: $\mathrm{R}_{\mathrm{f}}=$ 0.14 (80\% EtOAc/Hex). IR (FT-ATR, neat): 1649, 1540, 1498, 1419, 1324, 1299, $1212,1175,1110,726 \mathrm{~cm}^{-1} .{ }^{1} \mathrm{H}$ NMR $\left(600 \mathrm{MHz}, \mathrm{CDCl}_{3}\right) \delta 8.41(\mathrm{~d}, J=4.6 \mathrm{~Hz}, 1 \mathrm{H})$, $8.16(\mathrm{~d}, J=9.1 \mathrm{~Hz}, 2 \mathrm{H}), 7.52(\mathrm{~d}, J=9.1 \mathrm{~Hz}, 2 \mathrm{H}), 7.46(\mathrm{~d}, J=7.6 \mathrm{~Hz}, 1 \mathrm{H}), 7.19(\mathrm{~s}, 1 \mathrm{H}), 7.16(\mathrm{dd}, J=8.2,1.7 \mathrm{~Hz}$, 
$1 \mathrm{H}), 7.13(\mathrm{dd}, J=8.0,3.6 \mathrm{~Hz}, 2 \mathrm{H}), 6.78(\mathrm{~d}, J=3.9 \mathrm{~Hz}, 1 \mathrm{H}), 3.79(\mathrm{ddd}, J=19.2,12.1,5.2 \mathrm{~Hz}, 2 \mathrm{H}), 3.43-3.29$ (m, $4 \mathrm{H}), 2.91-2.76(\mathrm{~m}, 2 \mathrm{H}), 2.68(\mathrm{ddd}, J=14.0,8.9,4.5 \mathrm{~Hz}, 1 \mathrm{H}), 2.52(\mathrm{ddd}, J=13.9,9.1,4.6 \mathrm{~Hz}, 1 \mathrm{H}), 2.42(\mathrm{dq}, J=$ $15.2,5.8 \mathrm{~Hz}, 2 \mathrm{H}) .{ }^{13} \mathrm{C}$ NMR $\left(151 \mathrm{MHz}, \mathrm{CDCl}_{3}\right) \delta 156.7,153.7,146.9,145.5,142.6,139.7,137.9,137.6,136.1$, 135.2, 133.5, 133.3, 130.5, 129.2, 126.4, 125.2, 122.6, 118.5, 77.2, 45.1, 45.0, 31.8, 31.7, 30.6, 30.3. HRMS (ESI) $\mathrm{m} / \mathrm{z}:[\mathrm{M}+\mathrm{H}]^{+}$calcd for $\mathrm{C}_{26} \mathrm{H}_{24} \mathrm{ClN}_{4} \mathrm{O}_{3}{ }^{+} 475.131$, found 475.1548 .

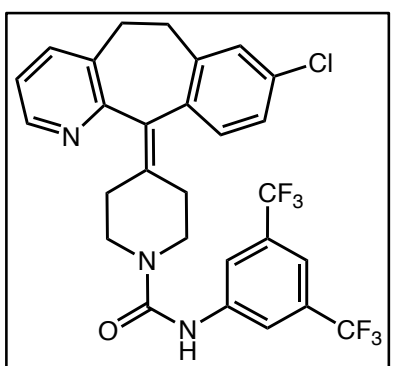

$N$-(3,5-bis(trifluoromethyl)phenyl)-4-(8-chloro-5,6-dihydro-11 $H$-benzo[5,6]cyclohepta $[1,2-b]$ pyridin-11-ylidene)piperidine-1-carboxamide (1h)

Using the general procedure with 3,5-bistrifluoromethylphenyl isocyanate $(0.12 \mathrm{~mL}$, $0.65 \mathrm{mmol}, 1.30$ equiv), the crude product was purified using normal-phase column chromatography (SNAP Ultra $50 \mathrm{~g}$, gradient $=0-10 \% \mathrm{DCM} / \mathrm{MeOH}$ for $10 \mathrm{CV}$ ) to afford a white solid (0.275 g, $0.49 \mathrm{mmol}, 97 \%$ yield). TLC: $\mathrm{R}_{\mathrm{f}}=0.81(80 \% \mathrm{EtOAc} / \mathrm{Hex})$. IR (FT-ATR, neat): 1645, 1558, 1373, 1274, 1170, 1124, $682 \mathrm{~cm}^{-1} .{ }^{1} \mathrm{H}$ NMR (500 $\left.\mathrm{MHz}, \mathrm{CD}_{3} \mathrm{OD}\right) \delta 8.34(\mathrm{~d}, J=4.5 \mathrm{~Hz}, 1 \mathrm{H}), 8.06(\mathrm{~s}, 2 \mathrm{H}), 7.67(\mathrm{~d}, J=7.7 \mathrm{~Hz}, 1 \mathrm{H}), 7.51$ $(\mathrm{s}, 1 \mathrm{H}), 7.28(\mathrm{dd}, J=7.5,5.1 \mathrm{~Hz}, 1 \mathrm{H}), 7.25(\mathrm{~s}, 1 \mathrm{H}), 7.19(\mathrm{~d}, J=8.3 \mathrm{~Hz}, 1 \mathrm{H}), 7.15(\mathrm{~d}, J=8.1 \mathrm{~Hz}, 1 \mathrm{H}), 3.90-3.76$ $(\mathrm{m}, 2 \mathrm{H}), 3.48-3.38(\mathrm{~m}, 2 \mathrm{H}), 3.38-3.32(\mathrm{~m}, 2 \mathrm{H}), 2.94-2.79(\mathrm{~m}, 2 \mathrm{H}), 2.50(\mathrm{td}, J=9.5,4.4 \mathrm{~Hz}, 2 \mathrm{H}), 2.40(\mathrm{dt}, J$ $=13.6,4.0 \mathrm{~Hz}, 1 \mathrm{H}), 2.27(\mathrm{dt}, J=14.0,4.1 \mathrm{~Hz}, 1 \mathrm{H}) .{ }^{13} \mathrm{C} \mathrm{NMR}\left(151 \mathrm{MHz}, \mathrm{CD}_{3} \mathrm{OD}\right) \delta 158.3,156.7,147.1,143.6$, 141.2, 139.7, 138.6, 138.5, 135.9, 135.0, $134.3,132.9$ (q, $J=33.0 \mathrm{~Hz}), 131.7,130.4,127.1,124.9$ (q, $J=271.8 \mathrm{~Hz})$, 124.2, $120.6(\mathrm{~d}, J=3.5 \mathrm{~Hz}) 116.1-115.9(\mathrm{~m}), 46.0,45.9,32.6,32.0,31.7,31.6 .{ }^{19} \mathrm{~F}$ NMR $\left(470 \mathrm{MHz}, \mathrm{CD}_{3} \mathrm{OD}\right) \delta$ -64.63. HRMS (ESI) $\mathrm{m} / \mathrm{z}:[\mathrm{M}+\mathrm{H}]^{+}$calcd for $\mathrm{C}_{28} \mathrm{H}_{23} \mathrm{ClF}_{6} \mathrm{~N}_{3} \mathrm{O}^{+}$566.1428, found 566.1446 .

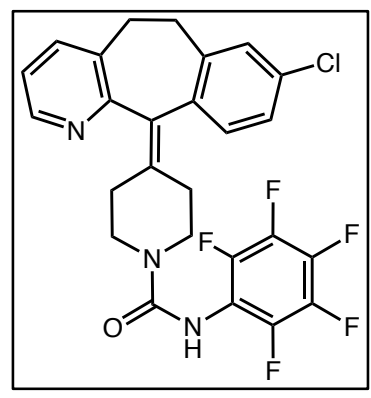

\section{4-(8-chloro-5,6-dihydro-11H-benzo[5,6]cyclohepta[1,2-b]pyridin-11-ylidene)-} $N$-(perfluorophenyl)piperidine-1-carboxamide (1i)

Using the general procedure with pentafluorophenyl isocynate $(0.11 \mathrm{~mL}, 0.83 \mathrm{mmol}$, 1.30 equiv), the crude product was purified using normal-phase column chromatography (SNAP Ultra 50g, gradient $=0-15 \% \mathrm{DCM} / \mathrm{MeOH}$ for $10 \mathrm{CV}$ ) to afford a white solid (0.3063 g, $0.59 \mathrm{mmol}$, 92\% yield). TLC: $\mathrm{R}_{\mathrm{f}}=0.89(10 \% \mathrm{MeOH} / \mathrm{EtOAc})$. IR (FTATR, neat): 1640, 1521, 1489, 1464, 1438, 1231, $991 \mathrm{~cm}^{-1} .{ }^{1} \mathrm{H}$ NMR $(600 \mathrm{MHz}$, $\left.\mathrm{CDCl}_{3}\right) \delta 8.41(\mathrm{~d}, J=3.9 \mathrm{~Hz}, 1 \mathrm{H}), 7.47(\mathrm{~d}, J=7.5 \mathrm{~Hz}, 1 \mathrm{H}), 7.19(\mathrm{~s}, 1 \mathrm{H}), 7.17-7.11$ $(\mathrm{m}, 3 \mathrm{H}), 6.03(\mathrm{~s}, 1 \mathrm{H}), 3.79$ (ddt, $J=17.9,11.1,5.0 \mathrm{~Hz}, 2 \mathrm{H}), 3.43-3.27(\mathrm{~m}, 4 \mathrm{H}), 2.92$ $-2.77(\mathrm{~m}, 2 \mathrm{H}), 2.66(\mathrm{ddd}, J=14.0,9.0,4.5 \mathrm{~Hz}, 1 \mathrm{H}), 2.51(\mathrm{ddd}, J=13.9,9.1,4.6 \mathrm{~Hz}, 1 \mathrm{H}), 2.46-2.37(\mathrm{~m}, 2 \mathrm{H}) .{ }^{13} \mathrm{C}$ $\operatorname{NMR}\left(151 \mathrm{MHz}, \mathrm{CDCl}_{3}\right) \delta 156.7,153.8,146.8,142.8(\mathrm{ddq}, J=243.0,11.6,4.1 \mathrm{~Hz}), 140.36-138.17(\mathrm{~m}), 138.88$ - 136.81 (m), 139.7, 138.0, 137.6, 136.4, 135.0, 133.6, 133.3, 130.5, 129.2, 126.4, 122.6, 114.12 (td, $J=15.0,14.5$, $3.7 \mathrm{~Hz}), 45.4,45.4,31.8,31.7,30.5,30.3 .{ }^{19} \mathrm{~F} \mathrm{NMR}\left(471 \mathrm{MHz} \mathrm{CDCl}_{3}\right) \delta-144.00--153.24(\mathrm{~m}),-159.07(\mathrm{t}, J=21.6$ $\mathrm{Hz}$ ), $-163.16\left(\mathrm{td}, J=22.6,6.0 \mathrm{~Hz}\right.$ ). HRMS (ESI) $\mathrm{m} / z:[\mathrm{M}+\mathrm{H}]^{+}$calcd for $\mathrm{C}_{26} \mathrm{H}_{20} \mathrm{ClF}_{5} \mathrm{~N}_{3} \mathrm{O}^{+} 520.1210$, found 520.1208 .

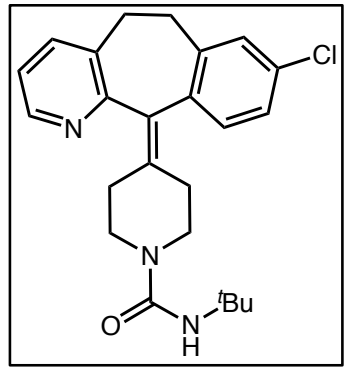

$N$-( $t$-Butyl)-4-(8-chloro-5,6-dihydro-11 $H$-benzo[5,6]cyclohepta[1,2-b]pyridin-11ylidene)piperidine-1-carboxamide ( $1 \mathrm{j})$

Using the general procedure with $t$-butyl isocyanate $(0.12 \mathrm{~mL}, 1.30 \mathrm{mmol}, 1.30$ equiv $)$, the crude product was purified using reverse-phase column chromatography (SNAP Ultra $\mathrm{C} 1860 \mathrm{~g}$, gradient $=30-80 \%$ acetonitrile $/ \mathrm{H}_{2} \mathrm{O}$ for $\left.9 \mathrm{CV}\right)$ to afford a white solid $(0.410$ g, $1.00 \mathrm{mmol}$, quant. yield). TLC: $\mathrm{R}_{\mathrm{f}}=0.33$ (80\% EtOAc/Hex). IR (FT-ATR, neat): 1630, 1523, 1437, 1391, 1207, 989, 827, $754 \mathrm{~cm}^{-1} .{ }^{1} \mathrm{H}$ NMR $\left(600 \mathrm{MHz}, \mathrm{CDCl}_{3}\right) \delta 8.41$ $(\mathrm{d}, J=4.3 \mathrm{~Hz}, 1 \mathrm{H}), 7.46(\mathrm{bs}, 1 \mathrm{H}), 7.17(\mathrm{~s}, 1 \mathrm{H}), 7.14(\mathrm{~s}, 3 \mathrm{H}), 4.29(\mathrm{~s}, 1 \mathrm{H}), 3.63(\mathrm{dt}, J=$ 10.6, $4.7 \mathrm{~Hz}, 2 \mathrm{H}), 3.42-3.31(\mathrm{~m}, 2 \mathrm{H}), 3.10-3.04(\mathrm{~m}, 2 \mathrm{H}), 2.89-2.82(\mathrm{~m}, 1 \mathrm{H}), 2.83-$ $2.75(\mathrm{~m}, 1 \mathrm{H}), 2.63-2.52(\mathrm{~m}, 1 \mathrm{H}), 2.42(\mathrm{ddd}, J=13.9,9.4,4.5 \mathrm{~Hz}, 1 \mathrm{H}), 2.34(\mathrm{ddt}, J=13.8,9.5,4.7 \mathrm{~Hz}, 2 \mathrm{H}), 1.34$ (s, 9H). ${ }^{13} \mathrm{C}$ NMR $\left(151 \mathrm{MHz}, \mathrm{CDCl}_{3}\right) \delta 157.1,157.0,146.7,139.6,137.8,137.8,137.7,134.0,133.5,133.0,130.7$, 
129.1, 126.3, 122.4, 50.9, 44.9, 44.7, 31.8, 31.6, 30.6, 30.4, 29.6. HRMS (ESI) $\mathrm{m} / z:[\mathrm{M}+\mathrm{H}]^{+}$calcd for $\mathrm{C}_{24} \mathrm{H}_{29} \mathrm{ClN}_{3} \mathrm{O}^{+} 410.1994$, found 410.1992 .

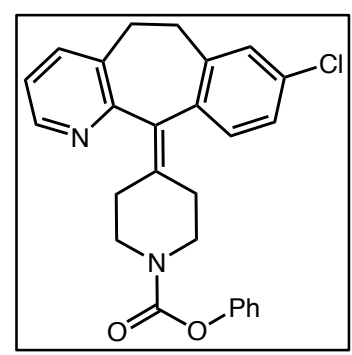

Phenyl 4-(8-chloro-5,6-dihydro-11H-benzo[5,6]cyclohepta[1,2-b]pyridin-11-ylidene)piperidine-1-carboxylate $(1 \mathrm{k})$

To a 5-dram vial equipped with a stir bar, desloratadine $(0.311 \mathrm{~g}, 1.00 \mathrm{mmol}, 1.00$ equiv $)$ was added and dissolved in THF/ $\mathrm{H}_{2} \mathrm{O}(1: 1 \mathrm{v} / \mathrm{v}, 10 \mathrm{~mL})$. Then, phenyl chloroformate $\left(0.16 \mathrm{~mL}, 1.30 \mathrm{mmol}, 1.30\right.$ equiv) was added followed by $\mathrm{K}_{2} \mathrm{CO}_{3}(0.180 \mathrm{~g}, 1.30 \mathrm{mmol}$, 1.30 equiv). The reaction was allowed to stir for $15 \mathrm{~h}$ at room temperature at which point the solution was concentrated in vacuo. The crude material was purified using reversephase column chromatography (SNAP Ultra C18 60g; gradient $=30-100 \%$ $\mathrm{MeCN} / \mathrm{H}_{2} \mathrm{O}$ for $\left.10 \mathrm{CV}\right)$ to afford a white solid $\left(0.349 \mathrm{~g}, 0.81 \mathrm{mmol}, 81 \%\right.$ yield). TLC: $\mathrm{R}_{\mathrm{f}}=0.71$ ( $80 \%$ EtOAc/Hex). IR (FT-ATR, neat): 1726, 1420, 1203, 996, $746 \mathrm{~cm}^{-1} .{ }^{1} \mathrm{H}$ NMR $\left(600 \mathrm{MHz}, \mathrm{CD}_{3} \mathrm{OD}\right) \delta 8.34(\mathrm{~d}, J=$ $4.8 \mathrm{~Hz}, 1 \mathrm{H}), 7.67(\mathrm{~d}, J=7.7 \mathrm{~Hz}, 1 \mathrm{H}), 7.36(\mathrm{t}, J=7.9 \mathrm{~Hz}, 2 \mathrm{H}), 7.27(\mathrm{dd}, J=7.7,4.9 \mathrm{~Hz}, 1 \mathrm{H}), 7.25-7.23(\mathrm{~m}, 1 \mathrm{H})$, $7.21(\mathrm{t}, J=6.0 \mathrm{~Hz}, 1 \mathrm{H}), 7.19-7.18(\mathrm{~m}, 1 \mathrm{H}), 7.15(\mathrm{~d}, J=8.2 \mathrm{~Hz}, 1 \mathrm{H}), 7.09(\mathrm{~d}, J=8.0 \mathrm{~Hz}, 2 \mathrm{H}), 4.01-3.93(\mathrm{~m}, 1 \mathrm{H})$, $3.87-3.78(\mathrm{~m}, 1 \mathrm{H}), 3.51-3.38(\mathrm{~m}, 3 \mathrm{H}), 3.36-3.24(\mathrm{~m}, 1 \mathrm{H}), 2.88$ (dddd, $J=19.1,15.3,9.0,4.7 \mathrm{~Hz}, 2 \mathrm{H}), 2.56-$ $2.45(\mathrm{~m}, 2 \mathrm{H}), 2.44-2.34(\mathrm{~m}, 1 \mathrm{H}), 2.29(\mathrm{t}, J=16.3 \mathrm{~Hz}, 1 \mathrm{H}) .{ }^{13} \mathrm{C}$ NMR $\left(151 \mathrm{MHz}, \mathrm{CD}_{3} \mathrm{OD}\right) \delta 158.3,155.4,152.8$, 147.1, 141.2, 139.8, 138.4, 138.4, 135.9, 135.1, 134.3, 131.7, 130.4, 130.4, 127.1, 126.5, 124.2, 122.9, 46.3 (d, $J=$ $19.9 \mathrm{~Hz}), 46.0(\mathrm{~d}, J=20.9 \mathrm{~Hz}), 32.6,32.0,31.7(\mathrm{~d}, J=14.4 \mathrm{~Hz}), 31.4(\mathrm{~d}, J=14.6 \mathrm{~Hz})$. HRMS $(\mathrm{ESI}) \mathrm{m} / \mathrm{z}:[\mathrm{M}+\mathrm{H}]^{+}$ calcd for $\mathrm{C}_{26} \mathrm{H}_{24} \mathrm{ClN}_{2} \mathrm{O}_{2}{ }^{+} 431.1521$, found 431.1526 .

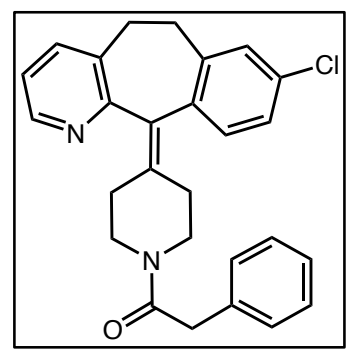

1-(4-(8-chloro-5,6-dihydro-11 $H$-benzo[5,6]cyclohepta[1,2-b]pyridin-11-ylidene)piperidin-1-yl)-2-phenylethan-1-one (11)

To a $10 \mathrm{~mL}$ round bottom flask equipped with a stir bar, desloratadine $(0.155 \mathrm{~g}, 0.50 \mathrm{mmol}$, 1.00 equiv) was dissolved in dichloromethane $(2.5 \mathrm{~mL})$, then 2 -phenylacetyl chloride $(0.10 \mathrm{~mL}, 0.75 \mathrm{mmol}, 1.50$ equiv) was added. The solution was allowed to stir for $7 \mathrm{~h}$ at room temperature, at which point it was concentrated in vacuo. The crude material was purified using reverse-phase column chromatography (SNAP Ultra C18 60g; gradient $=0$ $89 \% \mathrm{MeOH} / \mathrm{H}_{2} \mathrm{O}$ for $5 \mathrm{CV}$, then $89 \% \mathrm{MeOH} / \mathrm{H}_{2} \mathrm{O}$ for $2 \mathrm{CV}$, then $89-100 \% \mathrm{MeOH} / \mathrm{H}_{2} \mathrm{O}$ for $1 \mathrm{CV})$ to afford a white solid $\left(0.2107 \mathrm{~g}, 0.49 \mathrm{mmol}, 98 \%\right.$ yield). TLC: $\mathrm{R}_{\mathrm{f}}=0.78$ (10\% MeOH/EtOAc). IR (FTATR, neat): 1634, 1436, 1274, 1171, 994, 827, 723, $696 \mathrm{~cm}^{-1} .{ }^{1} \mathrm{H}$ NMR $\left(600 \mathrm{MHz}, \mathrm{CDCl}_{3}\right) \delta 8.38$ (dd, $J=18.5$, $4.1 \mathrm{~Hz}, 1 \mathrm{H}), 7.47-7.39(\mathrm{~m}, 2 \mathrm{H}), 7.33-7.27(\mathrm{~m}, 3 \mathrm{H}), 7.25-7.18(\mathrm{~m}, 1 \mathrm{H}), 7.16-7.01(\mathrm{~m}, 4 \mathrm{H}), 4.17-4.04(\mathrm{~m}$, $1 \mathrm{H}), 3.75(\mathrm{~s}, 2 \mathrm{H}), 3.66(\mathrm{ddq}, J=17.8,9.8,4.8 \mathrm{~Hz}, 1 \mathrm{H}), 3.39-3.25(\mathrm{~m}, 2 \mathrm{H}), 3.22-3.07(\mathrm{~m}, 2 \mathrm{H}), 2.86-2.72(\mathrm{~m}$, $2 \mathrm{H}), 2.45\left(\mathrm{dt}, J=9.8,4.6 \mathrm{~Hz}, 0.5 \mathrm{H}^{*}\right), 2.37-2.27(\mathrm{~m}, 2 \mathrm{H}), 2.15(\mathrm{dt}, J=14.0,4.3 \mathrm{~Hz}, 1 \mathrm{H}), 2.08(\mathrm{ddd}, J=14.0,9.5$, $\left.4.5 \mathrm{~Hz}, 0.5 \mathrm{H}^{*}\right) .{ }^{13} \mathrm{C}$ NMR $\left(151 \mathrm{MHz}, \mathrm{CDCl}_{3}\right) \delta 169.5 / 169.5$ (1C), 157.0/156.7 (1C), 146.8/146.7 (1C), 139.7/139.5 (1C), 137.7/137.7 (1C), 137.5, 137.0/137.0 (1C), 135.2, 134.6, 133.5/133.4 (1C), 133.1/133.1 (1C), 130.6/130.5 (1C), 129.1/129.0 (1C), 128.9, 128.6, 126.9, 126.4/126.2 (1C), 122.5/122.4 (1C), 47.0, 43.0/43.0 (1C), 41.5/41.4 (1C), 31.7/31.7 (1C), 31.6/31.6 (1C), 31.0/30.9 (1C), 30.6/30.3 (1C). [*Note: two conformations were detected via ${ }^{1} \mathrm{H}$ and ${ }^{13} \mathrm{C}$ NMR $]$. HRMS (ESI) $\mathrm{m} / z$ : $[\mathrm{M}+\mathrm{H}]^{+}$calcd for $\mathrm{C}_{27} \mathrm{H}_{26} \mathrm{ClN}_{2} \mathrm{O}^{+}$ 429.1728 , found 429.1745 .

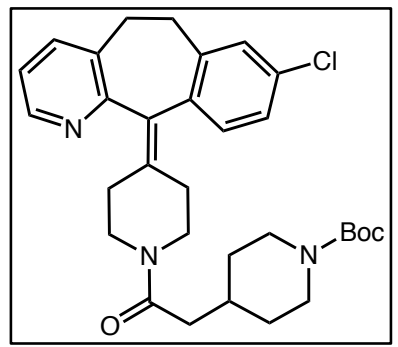

$t$-Butyl 4-(2-(4-(8-chloro-5,6-dihydro-11H-benzo[5,6]cyclohepta[1,2-b]-pyridin-11-ylidene)piperidin-1-yl)-2-oxoethyl)piperidine-1-carboxylate (1m)

To a $10 \mathrm{~mL}$ round bottom flask equipped with a stir bar, desloratadine $(0.155 \mathrm{~g}, 0.50$ mmol, 1.00 equiv) and $N$-Boc piperidinylacetic acid $(0.146 \mathrm{~g}, 0.60 \mathrm{mmol}, 1.20$ equiv) were added and dissolved in dichloromethane. While stirring at room temperature, HATU $(0.228 \mathrm{~g}, 0.60 \mathrm{mmol}, 1.20$ equiv $)$ was added followed by DIPEA $(0.21 \mathrm{~mL}$, $1.20 \mathrm{mmol}, 2.40$ equiv). After 24 hours, the solution was concentrated in vacuo and subjected to reverse-phase column chromatography (SNAP Ultra C18 60g; gradient 
$=30-100 \% \mathrm{MeOH} / \mathrm{H}_{2} \mathrm{O}$ for $\left.10 \mathrm{CV}\right)$ to afford a white solid $(0.248 \mathrm{~g}, 0.46 \mathrm{mmol}, 93 \%$ yield $)$. TLC: $\mathrm{R}_{\mathrm{f}}=0.75$ ( $10 \%$ $\mathrm{MeOH} / \mathrm{EtOAc}$ ). IR (FT-ATR, neat): 1685, 1637, 1422, 1365, 1282, 1160, $994 \mathrm{~cm}^{-1} .{ }^{1} \mathrm{H} \mathrm{NMR}\left(600 \mathrm{MHz}, \mathrm{CDCl}_{3}\right)$ $\delta 8.40(\mathrm{~s}, 1 \mathrm{H}), 7.44(\mathrm{~d}, J=7.2 \mathrm{~Hz}, 1 \mathrm{H}), 7.17(\mathrm{~s}, 1 \mathrm{H}), 7.16-7.08(\mathrm{~m}, 3 \mathrm{H}), 4.15-3.98(\mathrm{~m}, 3 \mathrm{H}), 3.68(\mathrm{td}, J=13.9$, $6.7 \mathrm{~Hz}, 1 \mathrm{H}), 3.36(\mathrm{ddd}, J=23.1,15.4,8.9 \mathrm{~Hz}, 2 \mathrm{H}), 3.27-3.18(\mathrm{~m}, 1 \mathrm{H}), 3.18-3.09(\mathrm{~m}, 1 \mathrm{H}), 2.88-2.77(\mathrm{~m}, 2 \mathrm{H})$, $2.71(\mathrm{bs}, 2 \mathrm{H}), 2.57-2.44(\mathrm{~m}, 1 \mathrm{H}), 2.41-2.30(\mathrm{~m}, 3 \mathrm{H}), 2.30-2.18(\mathrm{~m}, 2 \mathrm{H}), 1.99(\mathrm{bs}, 1 \mathrm{H}), 1.71(\mathrm{t}, J=10.6 \mathrm{~Hz}$, 2H), 1.44 (s, 9H), $1.18-1.02(\mathrm{~m}, 2 \mathrm{H}) .{ }^{13} \mathrm{C}$ NMR (151 MHz, $\left.\mathrm{CDCl}_{3}\right) \delta 170.2 / 170.1$ (1C), 157.0/156.8 (1C), 154.9, 146.9/146.7 (1C), 139.7/139.6 (1C), 137.8/137.7 (1C), 137.6, 137.0/136.9 (1C), 134.7, 133.6/133.4 (1C), 133.2/133.2 (1C), 130.6/130.5 (1C), 129.1, 126.4/126.3 (1C), 122.5/122.5 (1C), 79.4, 46.7, 46.6, 42.8, 42.7, 39.7, 33.5/33.4 (1C), 32.4, 32.4, 31.8/31.8 (1C), 31.7/31.6 (1C), 31.5/31.4 (1C), 30.7/30.5 (1C), 28.6 [Note: two conformations were detected via ${ }^{13} \mathrm{C}$ NMR]. HRMS (ESI) $\mathrm{m} / \mathrm{z}$ : $[\mathrm{M}+\mathrm{H}]^{+}$calcd for $\mathrm{C}_{31} \mathrm{H}_{39} \mathrm{ClN}_{3} \mathrm{O}_{3}{ }^{+}$ 536.2674, found 536.2677. 


\section{Synthesis and Characterization of Loratadine $\mathrm{N}$-Oxide Analogs}<smiles>[R]C(=O)N1CCC(=C2c3ccc(Cl)cc3CCc3cccnc32)CC1</smiles>

1

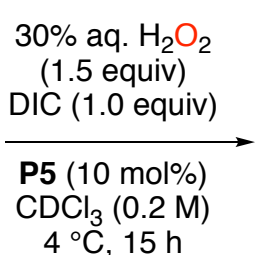

$4{ }^{\circ} \mathrm{C}, 15 \mathrm{~h}$

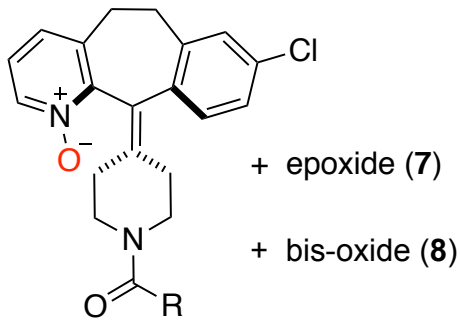

$(+)-6$

A. General Procedure: To a $2 \mathrm{~mL}$-vial equipped with a stir bar, loratadine analog 1 ( $0.05 \mathrm{mmol}, 1.00 \mathrm{equiv})$ and P5 $(2.9 \mathrm{mg}, 10 \mathrm{~mol} \%)$ were dissolved in $\mathrm{CDCl}_{3}(0.25 \mathrm{~mL}, 0.2 \mathrm{M})$. While vigorously stirring at $0{ }^{\circ} \mathrm{C}$ in an ice bath, $\mathrm{H}_{2} \mathrm{O}_{2}\left(30 \% \mathrm{w} / \mathrm{w}\right.$ in $\mathrm{H}_{2} \mathrm{O} ; 5.15 \mu \mathrm{L}, 0.05 \mathrm{mmol}, 1.50$ equiv) was added followed by DIC ( $7.74 \mu \mathrm{L}, 0.05 \mathrm{mmol}, 1.00$ equiv). The vial was sealed with a Teflon cap and transported to a cold room maintained at $4{ }^{\circ} \mathrm{C}$. After stirring for $15 \mathrm{~h}$, the reaction was quenched by addition of $\mathrm{Na}_{2} \mathrm{SO}_{3}$. The mixture was directly purified by normal-phase column chromatography (SNAP Ultra $25 \mathrm{~g}, 0-8 \% \mathrm{DCM} / \mathrm{MeOH}$ over $5 \mathrm{CV}$, then $8 \% \mathrm{DCM} / \mathrm{MeOH}$ for $2 \mathrm{CV}$, then $8-10 \%$ $\mathrm{DCM} / \mathrm{MeOH}$ for $2 \mathrm{CV}$ ) to afford the desired $N$-oxide unless otherwise stated.

Racemic standards were generated using $m$-CPBA (77\%; 1.00 equiv) in $\mathrm{CHCl}_{3}(0.2 \mathrm{M})$ for $15 \mathrm{~h}$ at rt.

\section{B. Determination of Reaction Conversion}
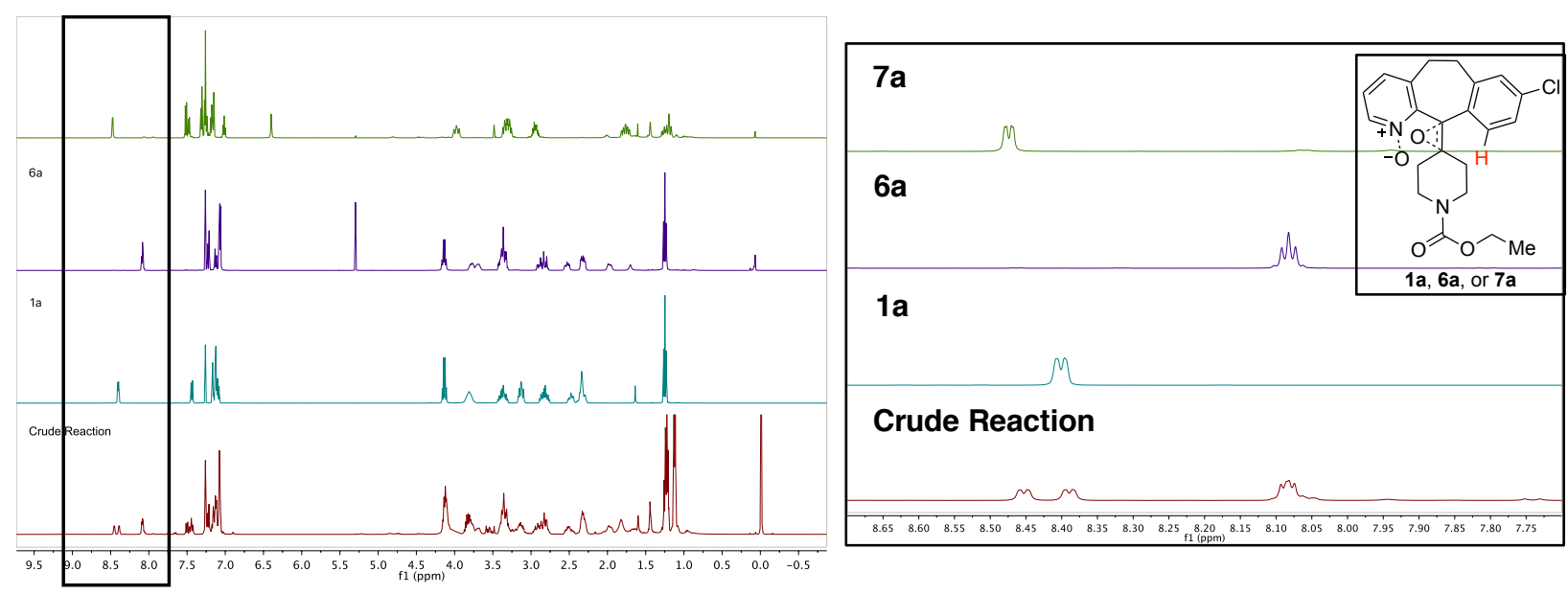

Figure S1. ${ }^{1} \mathrm{H}$ NMR $\left(\mathrm{CDCl}_{3}\right)$ NMRs of the crude reaction mixture (maroon), loratadine (1a, teal), loratadine $N$ oxide (6a, purple), and loratadine epoxide (7a, green). Conversion, defined as consumption of $1 \mathbf{a}$ to $6 \mathbf{a}$ and $7 \mathbf{a}$ (and $\mathbf{8 a}$ if detected), was determined by integrating the indicated proton (red) in the crude reaction. 


\section{C. $N$-Oxide Characterization}

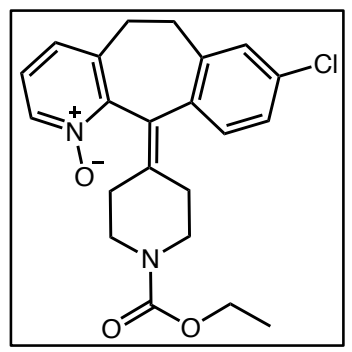

8-Chloro-11-(1-(ethoxycarbonyl)piperidin-4-ylidene)-6,11-dihydro-5 $H$-benzo[5, 6]cyclohepta $[1,2-b]$ pyridine 1-oxide (6a)

This known compound is commercially available (CAS: 165739-62-8). White solid (9.5 $\mathrm{mg}, 0.024 \mathrm{mmol}, 48 \%$ yield). HPLC (Chiralpak IB column, 80\% EtOH/hexanes, 0.7 $\mathrm{mL} / \mathrm{min}$ flowrate, $2 \mu \mathrm{L}$ injection, $25^{\circ} \mathrm{C}, 254 \mathrm{~nm}$ ): minor enantiomer $t_{\mathrm{R}}=6.8 \mathrm{~min}$, major enantiomer $t_{\mathrm{R}}=7.7 \mathrm{~min} .[\boldsymbol{\alpha}]_{589}^{20}\left((-)-6 \mathrm{a} ; \mathrm{c}=0.14, \mathrm{CHCl}_{3} ; 98: 2 \mathrm{er}\right):-212.6 .[\boldsymbol{\alpha}]_{589}^{20}$ $\left((+)-6 \mathrm{a} ; \mathrm{c}=0.24, \mathrm{CHCl}_{3} ;>99: 1 \mathrm{er}\right):+184.6$
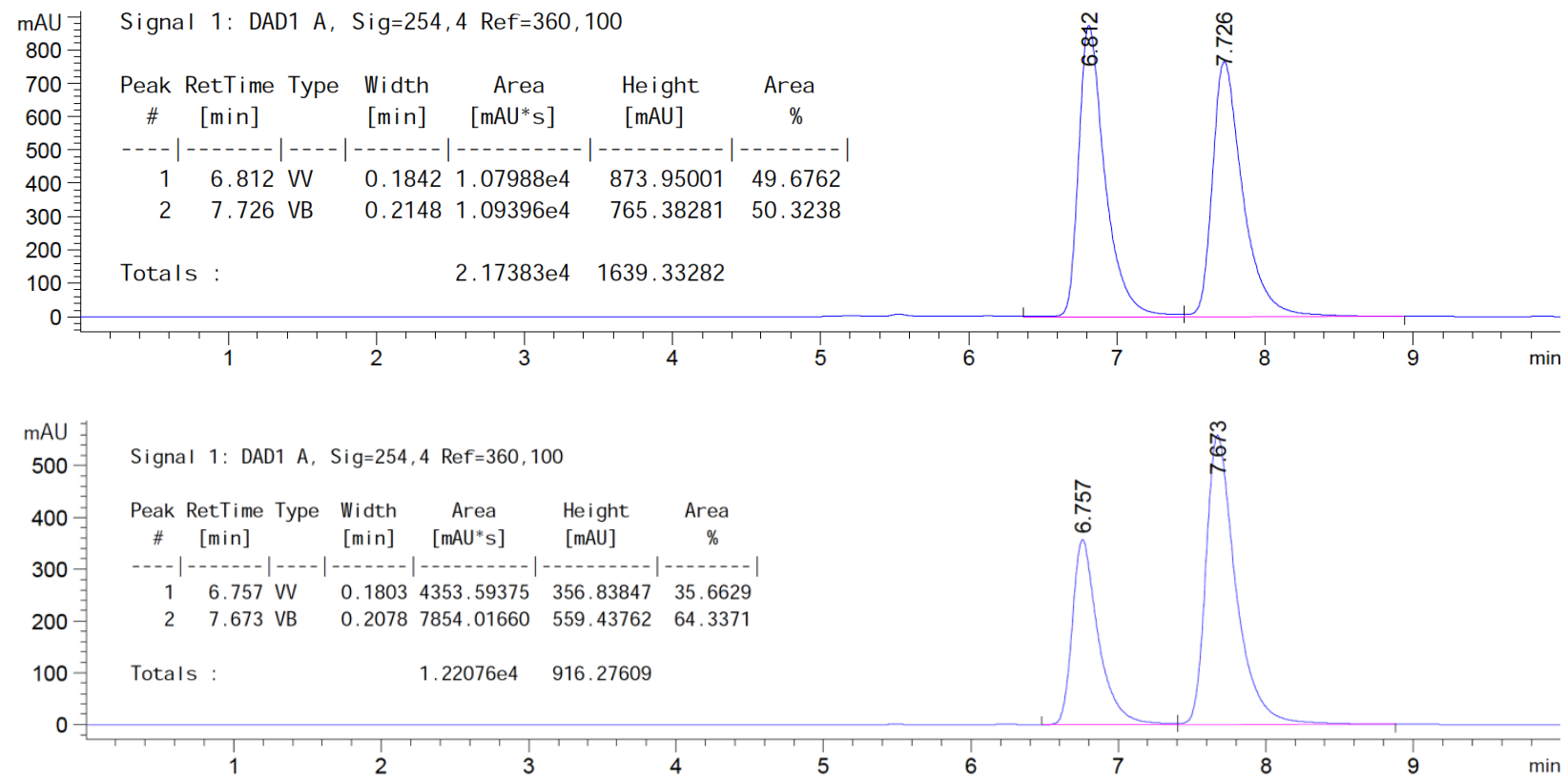


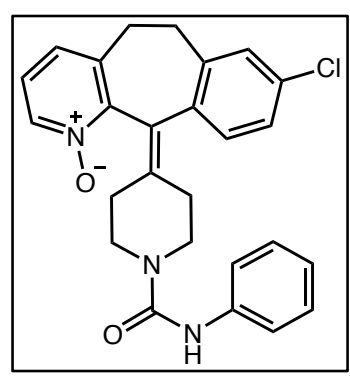

8-Chloro-11-(1-(phenylcarbamoyl)piperidin-4-ylidene)-6,11-dihydro-5H-benzo $[5,6]$ cyclohepta $[1,2 b]$-pyridine 1-oxide $(6 \mathrm{~b})$

White solid ( $8.9 \mathrm{mg}, 0.020 \mathrm{mmol}, 40 \%$ yield). TLC: $\mathrm{R}_{\mathrm{f}}=0.66(10 \% \mathrm{MeOH} / \mathrm{EtOAc})$. IR (FT-ATR, neat): 1635, 1593, 1537, 1441,1238, 991, $748 \mathrm{~cm}^{-1} .{ }^{1} \mathrm{H}$ NMR $(600 \mathrm{MHz}$, $\left.\mathrm{CD}_{3} \mathrm{OD}\right) \delta 8.19(\mathrm{dd}, J=6.5,1.1 \mathrm{~Hz}, 1 \mathrm{H}), 7.40-7.30(\mathrm{~m}, 5 \mathrm{H}), 7.27-7.23(\mathrm{~m}, 2 \mathrm{H}), 7.21$

$-7.16(\mathrm{~m}, 2 \mathrm{H}), 7.00(\mathrm{tt}, J=7.4,1.2 \mathrm{~Hz}, 1 \mathrm{H}), 3.81(\mathrm{tt}, J=11.2,5.3 \mathrm{~Hz}, 2 \mathrm{H}), 3.48-3.36$ $(\mathrm{m}, 4 \mathrm{H}), 3.00-2.86(\mathrm{~m}, 2 \mathrm{H}), 2.59(\mathrm{ddd}, J=13.2,8.5,4.4 \mathrm{~Hz}, 1 \mathrm{H}), 2.41(\mathrm{ddd}, J=14.3$, 6.2, $4.0 \mathrm{~Hz}, 1 \mathrm{H}), 2.33(\mathrm{ddt}, J=13.0,8.4,4.3 \mathrm{~Hz}, 1 \mathrm{H}), 2.03(\mathrm{ddd}, J=14.2,6.4,4.0 \mathrm{~Hz}$, $1 \mathrm{H}) .{ }^{13} \mathrm{C}$ NMR $\left(151 \mathrm{MHz}, \mathrm{CD}_{3} \mathrm{OD}\right) \delta 157.8,150.8,143.1,140.9,140.5,140.4,138.5,134.9,134.8,133.6,131.3$, 130.2, 129.6, 127.0, 126.2, 124.4, 124.1, 122.2, 45.8, 45.4, 32.9, 32.6, 31.4, 31.1. HRMS (ESI) $\mathrm{m} / \mathrm{z}:[\mathrm{M}+\mathrm{H}]^{+}$calcd for $\mathrm{C}_{26} \mathrm{H}_{25} \mathrm{ClN}_{3} \mathrm{O}_{2}{ }^{+} 446.1630$ found 446.1644. HPLC (Chiralpak IB column, $60 \% \mathrm{EtOH} /$ hexanes, $0.7 \mathrm{~mL} / \mathrm{min}$ flowrate, $2 \mu \mathrm{L}$ injection, $\left.25^{\circ} \mathrm{C}, 254 \mathrm{~nm}\right)$ : minor enantiomer $t_{\mathrm{R}}=7.7 \mathrm{~min}$, major enantiomer $t_{\mathrm{R}}=9.5 \mathrm{~min}$. $[\boldsymbol{\alpha}]_{589}^{20}(\mathrm{c}$ $\left.=0.57, \mathrm{CHCl}_{3} ; 93: 7 \mathrm{er}\right):+96.1$ X-ray characterization: recrystallized from ethanol.
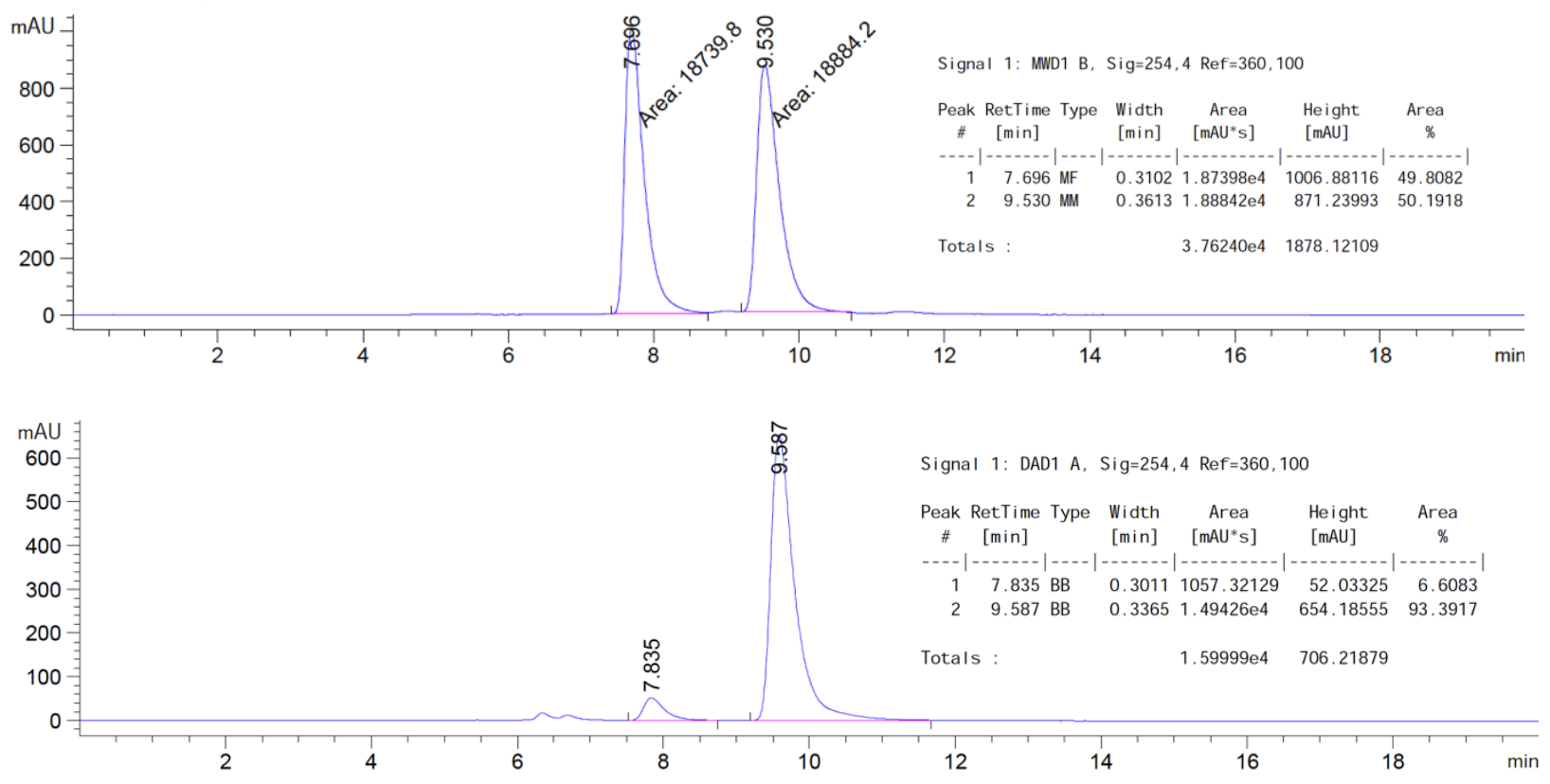


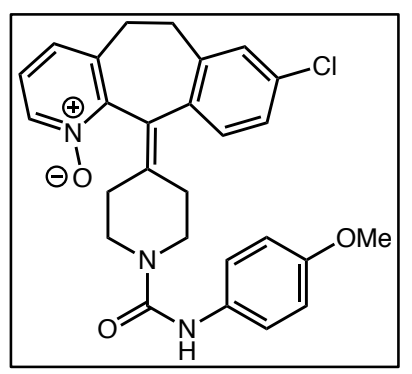

8-Chloro-11-(1-((4-methoxyphenyl)carbamoyl)piperidin-4-ylidene)-6,11-di hydro-5H-benzo $[5,6]$-cyclohepta $[1,2-b]$ pyridine 1-oxide (6c)

White solid (9.5 g, $0.020 \mathrm{mmol}, 40 \%$ yield). TLC: $\mathrm{R}_{f}=0.63(10 \% \mathrm{MeOH} / \mathrm{EtOAc})$. IR (FT-ATR, neat): 1633, 1510, 1417, 1225, 1173, 1031, 991, $824 \mathrm{~cm}^{-1} .{ }^{1} \mathrm{H}$ NMR $\left(600 \mathrm{MHz}, \mathrm{CD}_{3} \mathrm{OD}\right) \delta 8.19(\mathrm{~d}, J=6.4 \mathrm{~Hz}, 1 \mathrm{H}), 7.49(\mathrm{~d}, J=7.7 \mathrm{~Hz}, 1 \mathrm{H}), 7.37(\mathrm{dd}, J$ $=7.7,6.6 \mathrm{~Hz}, 1 \mathrm{H}), 7.35-7.29(\mathrm{~m}, 1 \mathrm{H}), 7.24-7.20(\mathrm{~m}, 2 \mathrm{H}), 7.20-7.17(\mathrm{~m}, 2 \mathrm{H})$, $6.86-6.80(\mathrm{~m}, 2 \mathrm{H}), 3.80(\mathrm{tt}, J=11.2,5.5 \mathrm{~Hz}, 2 \mathrm{H}), 3.75(\mathrm{~s}, 3 \mathrm{H}), 3.49-3.36(\mathrm{~m}, 4 \mathrm{H})$, $3.02-2.87(\mathrm{~m}, 2 \mathrm{H}), 2.58(\mathrm{ddd}, J=13.3,8.5,4.4 \mathrm{~Hz}, 1 \mathrm{H}), 2.40(\mathrm{ddd}, J=14.2,6.2,4.0$ $\mathrm{Hz}, 1 \mathrm{H}), 2.36-2.29(\mathrm{~m}, 1 \mathrm{H}), 2.02(\mathrm{ddd}, J=14.2,6.5,3.9 \mathrm{~Hz}, 1 \mathrm{H}) .{ }^{13} \mathrm{C} \mathrm{NMR}\left(151 \mathrm{MHz}, \mathrm{CD}_{3} \mathrm{OD}\right) \delta 156.8,156.1$, 149.3, 141.7, 139.1, 138.9, 137.1, 133.5, 133.4, 132.2, 132.2, 129.8, 128.8, 125.6, 124.7, 123.2, 122.9, 113.4, 54.4, 44.3, 43.9, 31.4, 31.4, 30.0, 29.7. HRMS (ESI) $\mathrm{m} / \mathrm{z}:[\mathrm{M}+\mathrm{H}]^{+}$calcd for $\mathrm{C}_{27} \mathrm{H}_{27} \mathrm{ClN}_{3} \mathrm{O}_{3}{ }^{+}$476.1735, found 476.1756. HPLC (Chiralpak IB column, $60 \% \mathrm{EtOH} /$ hexanes, $0.7 \mathrm{~mL} / \mathrm{min}$ flowrate, $2 \mu \mathrm{L}$ injection, $25^{\circ} \mathrm{C}, 254 \mathrm{~nm}$ ): minor enantiomer $t_{\mathrm{R}}=9.0 \mathrm{~min}$, major enantiomer $t_{\mathrm{R}}=11.4 \mathrm{~min}$. $[\boldsymbol{\alpha}]_{\mathbf{5 8 9}}^{\mathbf{2 0}}\left(\mathrm{c}=0.44, \mathrm{CHCl}_{3} ; 93: 7 \mathrm{er}\right):+125.3$.
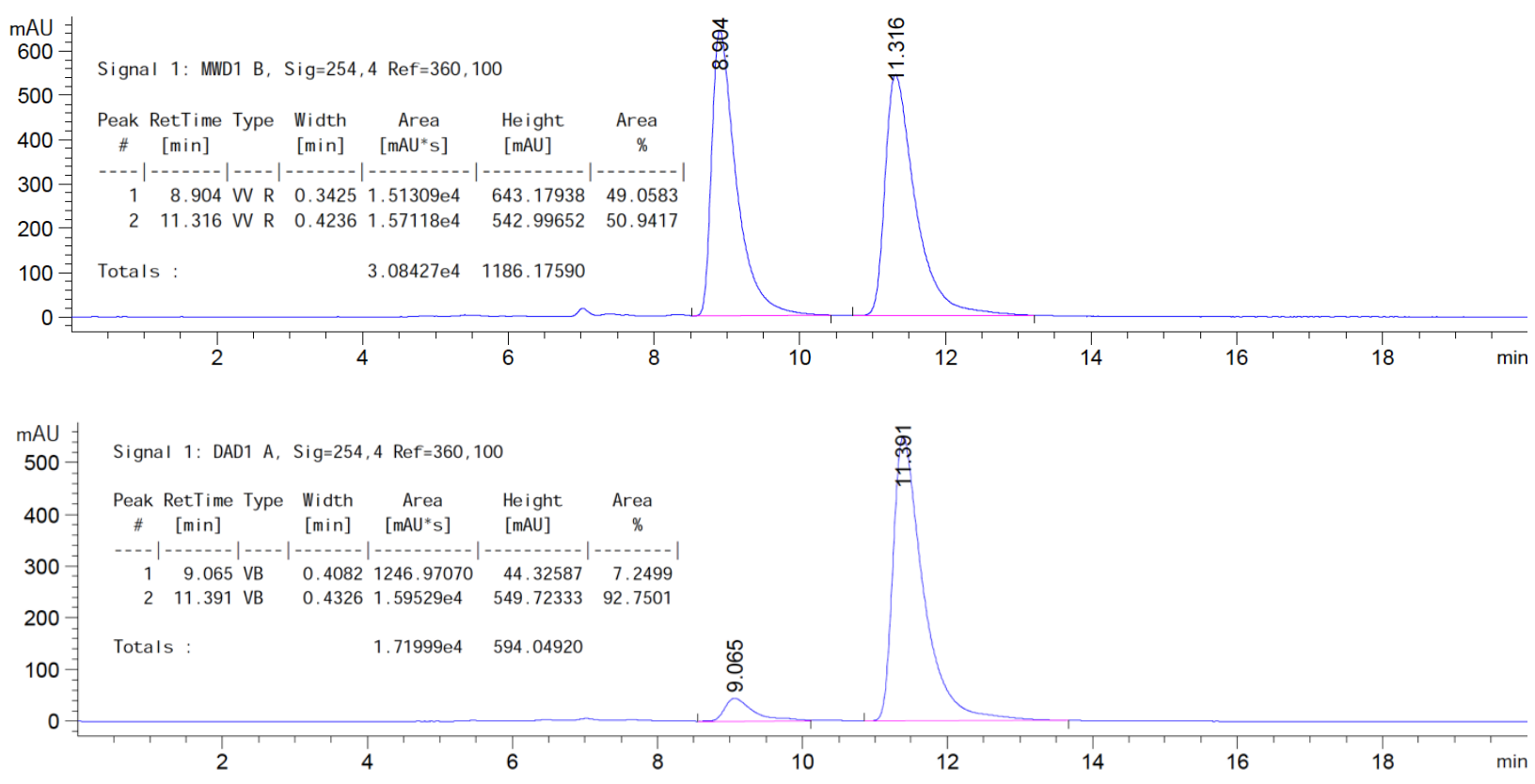


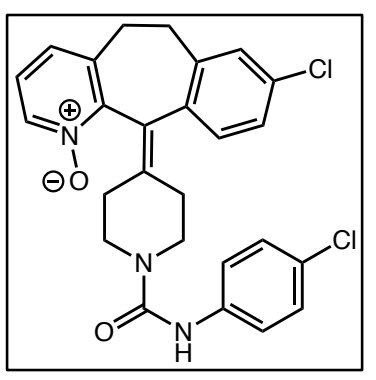

8-Chloro-11-(1-((4-chlorophenyl)carbamoyl)piperidin-4-ylidene)-6,11-dihydro$5 H$-benzo $[5,6]$ cyclohepta- $[1,2-b]$ pyridine 1 -oxide $(6 \mathrm{~d})$

White solid (10.8 mg, $0.023 \mathrm{mmol}$, $45 \%$ yield). TLC: $\mathrm{R}_{\mathrm{f}}=0.71$ (10\% MeOH/EtOAc). IR (FT-ATR, neat): 1634, 1592, 1492, 1429, 1406, 1237, 991, $823 \mathrm{~cm}^{-1} .{ }^{1} \mathrm{H}$ NMR $(600$ $\left.\mathrm{MHz}, \mathrm{CD}_{3} \mathrm{OD}\right) \delta 8.19(\mathrm{~d}, J=6.4 \mathrm{~Hz}, 1 \mathrm{H}), 7.49(\mathrm{~d}, J=7.7 \mathrm{~Hz}, 1 \mathrm{H}), 7.39-7.35(\mathrm{~m}$, $2 \mathrm{H}), 7.35-7.34(\mathrm{~m}, 1 \mathrm{H}), 7.33-7.30(\mathrm{~m}, 1 \mathrm{H}), 7.25-7.22(\mathrm{~m}, 2 \mathrm{H}), 7.20-7.17(\mathrm{~m}$, $2 \mathrm{H}), 3.80(\mathrm{tt}, J=11.8,5.4 \mathrm{~Hz}, 2 \mathrm{H}), 3.51-3.35(\mathrm{~m}, 4 \mathrm{H}), 3.00-2.85(\mathrm{~m}, 2 \mathrm{H}), 2.58$ (ddd, $J=13.3,8.5,4.4 \mathrm{~Hz}, 1 \mathrm{H}), 2.41$ (ddd, $J=14.2,6.1,4.0 \mathrm{~Hz}, 1 \mathrm{H}), 2.33$ (ddt, $J=12.9$, $8.4,4.3 \mathrm{~Hz}, 1 \mathrm{H}), 2.02(\mathrm{ddd}, J=14.2,6.5,4.0 \mathrm{~Hz}, 1 \mathrm{H}) .{ }^{13} \mathrm{C}$ NMR $\left(151 \mathrm{MHz}, \mathrm{CD}_{3} \mathrm{OD}\right) \delta 157.5,150.7,142.9,140.5$, 140.4, 139.9, 138.5, 134.9, 134.9, 133.6, 131.3, 130.2, 129.5, 128.9, 127.0, 126.2, 124.4, 123.3, 45.8, 45.4, 32.8, 32.8, 31.4, 31.1. HRMS (ESI) $\mathrm{m} / \mathrm{z}:[\mathrm{M}+\mathrm{H}]^{+}$calcd for $\mathrm{C}_{26} \mathrm{H}_{24} \mathrm{Cl}_{2} \mathrm{~N}_{3} \mathrm{O}_{2}{ }^{+} 480.1240$, found 480.1241. HPLC (Chiralpak IB column, $60 \% \mathrm{EtOH} /$ hexanes, $0.7 \mathrm{~mL} / \mathrm{min}$ flowrate, $2 \mu \mathrm{L}$ injection, $25^{\circ} \mathrm{C}, 254 \mathrm{~nm}$ ): minor enantiomer $t_{\mathrm{R}}=7.4 \mathrm{~min}$, major enantiomer $t_{\mathrm{R}}=8.3 \mathrm{~min}$. $[\alpha]_{589}^{20}\left(\mathrm{c}=0.55, \mathrm{CHCl}_{3} ; 96: 4 \mathrm{er}\right):+121.6$.
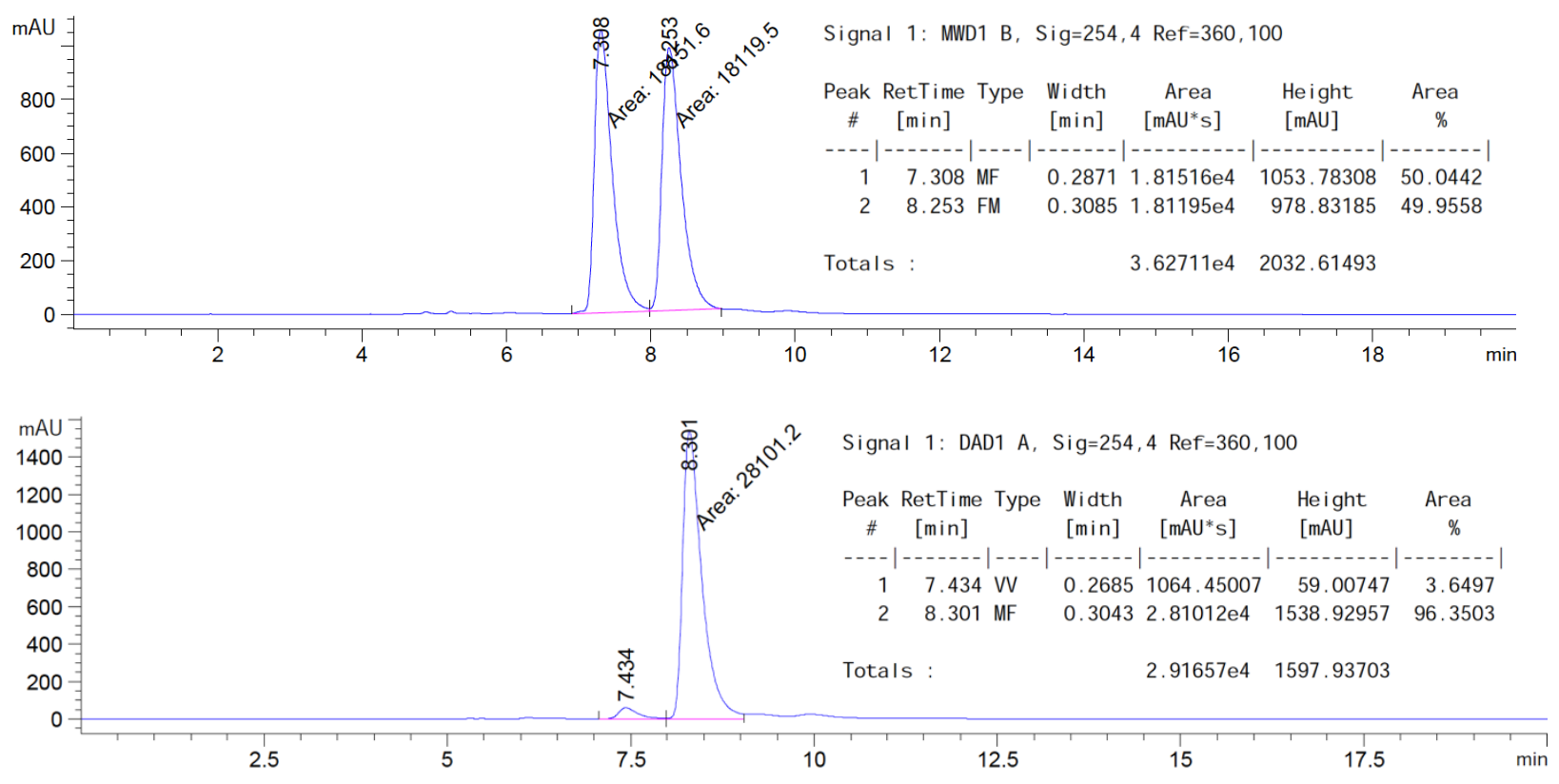


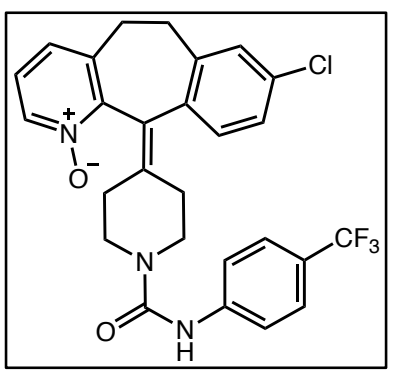

8-Chloro-11-(1-((4-trifluoromethylphenyl)carbamoyl)piperidin-4-ylidene)6,11-dihydro-5 H-benzo[5,6] cyclohepta-[1,2-b]pyridine 1-oxide (6e)

White solid (11.3 mg, $0.022 \mathrm{mmol}, 44 \%$ yield). The characterization of this $N$-oxide was previously described by Miller and co-workers. ${ }^{1}$ HPLC (Chiralpak IB column, $60 \% \mathrm{EtOH} /$ hexanes, $0.7 \mathrm{~mL} / \mathrm{min}$ flowrate, $2 \mu \mathrm{L}$ injection, $25^{\circ} \mathrm{C}, 254 \mathrm{~nm}$ ): minor enantiomer $t_{\mathrm{R}}=6.6 \mathrm{~min}$, major enantiomer $t_{\mathrm{R}}=7.3 \mathrm{~min}$.
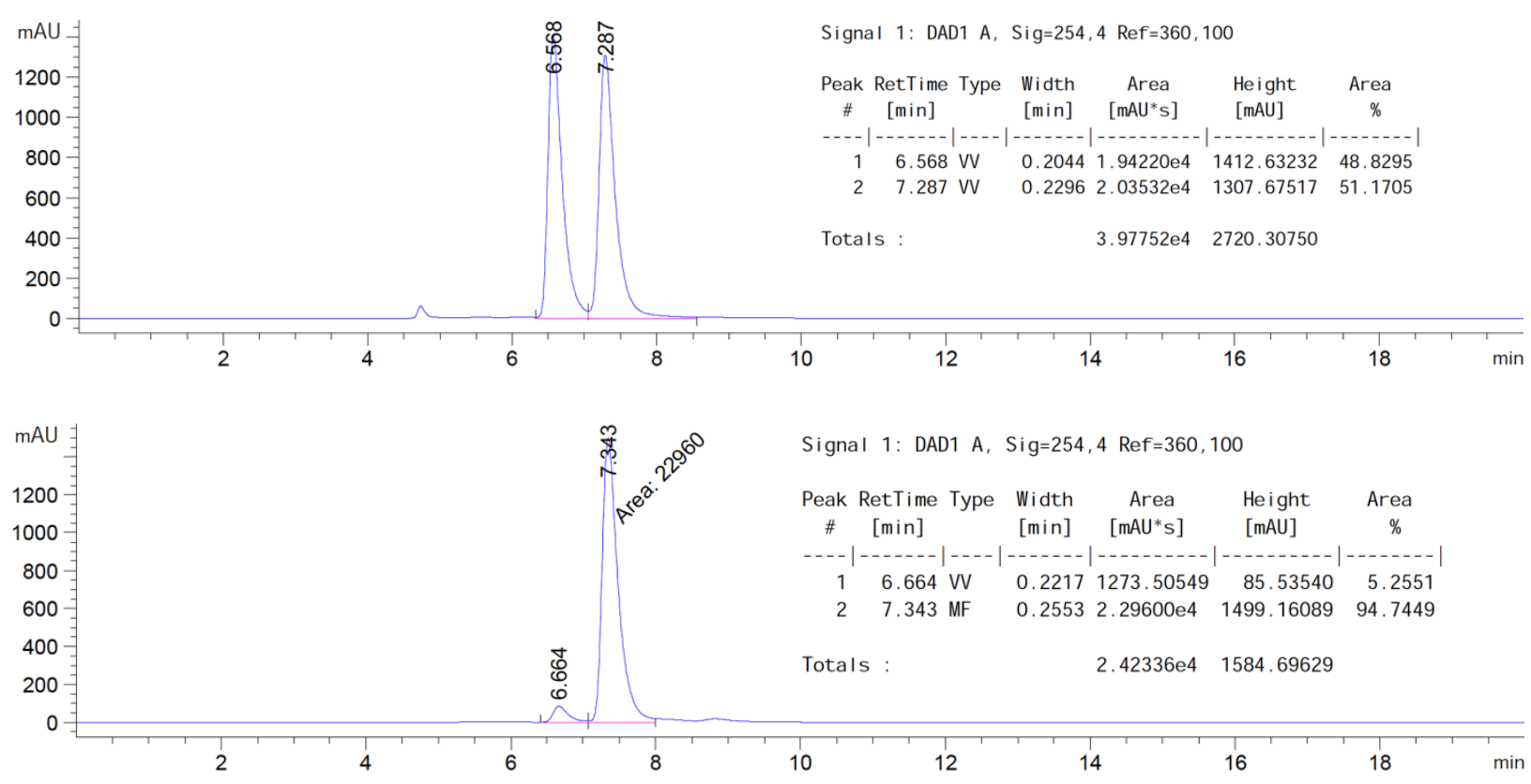
<smiles>O=C(Nc1ccc(Cl)cc1)N1CCC(=C2CC3CC4C=C2CC(C4)C3)c2ccc(Cl)cc21</smiles>

8-Chloro-11-(1-((4-cyanophenyl)carbamoyl)piperidin-4-ylidene)-6,11-dihy dro-5 $H$-benzo $[5,6]$ cyclohepta-[1,2-b]pyridine 1-oxide (6f)

White solid (11.3 mg, $0.24 \mathrm{mmol}, 48 \%$ yield). TLC: $\mathrm{R}_{\mathrm{f}}=0.68(10 \% \mathrm{MeOH} / \mathrm{EtOAc})$. IR (FT-ATR, neat): 2219, 1648, 1590, 1512, 1416, 1311, 1217, 1174, 991, $826 \mathrm{~cm}^{-1}$. ${ }^{1} \mathrm{H}$ NMR $\left(600 \mathrm{MHz}, \mathrm{CD}_{3} \mathrm{OD}\right) \delta 8.20(\mathrm{~d}, J=6.4 \mathrm{~Hz}, 1 \mathrm{H}), 7.61-7.56(\mathrm{~m}, 4 \mathrm{H}), 7.49$ $(\mathrm{d}, J=7.7 \mathrm{~Hz}, 1 \mathrm{H}), 7.37(\mathrm{dd}, J=7.7,6.6 \mathrm{~Hz}, 1 \mathrm{H}), 7.33-7.30(\mathrm{~m}, 1 \mathrm{H}), 7.21-7.17$ $(\mathrm{m}, 2 \mathrm{H}), 3.82$ (ddd, $J=18.2,9.1,4.8 \mathrm{~Hz}, 2 \mathrm{H}), 3.50-3.37(\mathrm{~m}, 4 \mathrm{H}), 2.98-2.88(\mathrm{~m}$, $2 \mathrm{H}), 2.60(\mathrm{ddd}, J=13.2,8.5,4.4 \mathrm{~Hz}, 1 \mathrm{H}), 2.42(\mathrm{ddd}, J=14.2,6.3,4.0 \mathrm{~Hz}, 1 \mathrm{H}), 2.33$ $(\mathrm{ddd}, J=14.0,8.3,4.3 \mathrm{~Hz}, 1 \mathrm{H}), 2.04(\mathrm{ddd}, J=14.2,6.6,4.0 \mathrm{~Hz}, 1 \mathrm{H}) .{ }^{13} \mathrm{C} \operatorname{NMR}\left(151 \mathrm{MHz}, \mathrm{CD}_{3} \mathrm{OD}\right) \delta 156.7,150.7$, 146.1, 142.8, 140.5, 140.4, 138.5, 134.9, 134.9, 133.9, 133.6, 131.3, 130.2, 127.0, 126.2, 124.5, 120.9, 120.2, 105.8, 45.9, 45.5, 32.8, 32.8, 31.4, 31.1. HRMS (ESI) $\mathrm{m} / \mathrm{z}:[\mathrm{M}+\mathrm{H}]^{+}$calcd for $\mathrm{C}_{27} \mathrm{H}_{24} \mathrm{ClN}_{4} \mathrm{O}_{2}{ }^{+} 471.1582$, found 471.1580 . HPLC (Chiralpak IB column, $60 \% \mathrm{EtOH} /$ hexanes, $0.7 \mathrm{~mL} / \mathrm{min}$ flowrate, $2 \mu \mathrm{L}$ injection, $25^{\circ} \mathrm{C}, 254 \mathrm{~nm}$ ): minor enantiomer $t_{\mathrm{R}}=8.0 \mathrm{~min}$, major enantiomer $t_{\mathrm{R}}=9.3 \mathrm{~min}$. $[\boldsymbol{\alpha}]_{589}^{20}\left(\mathrm{c}=0.57, \mathrm{CHCl}_{3} ; 96: 4 \mathrm{er}\right):+130.1$. X-ray characterization: recrystallized from ethanol.
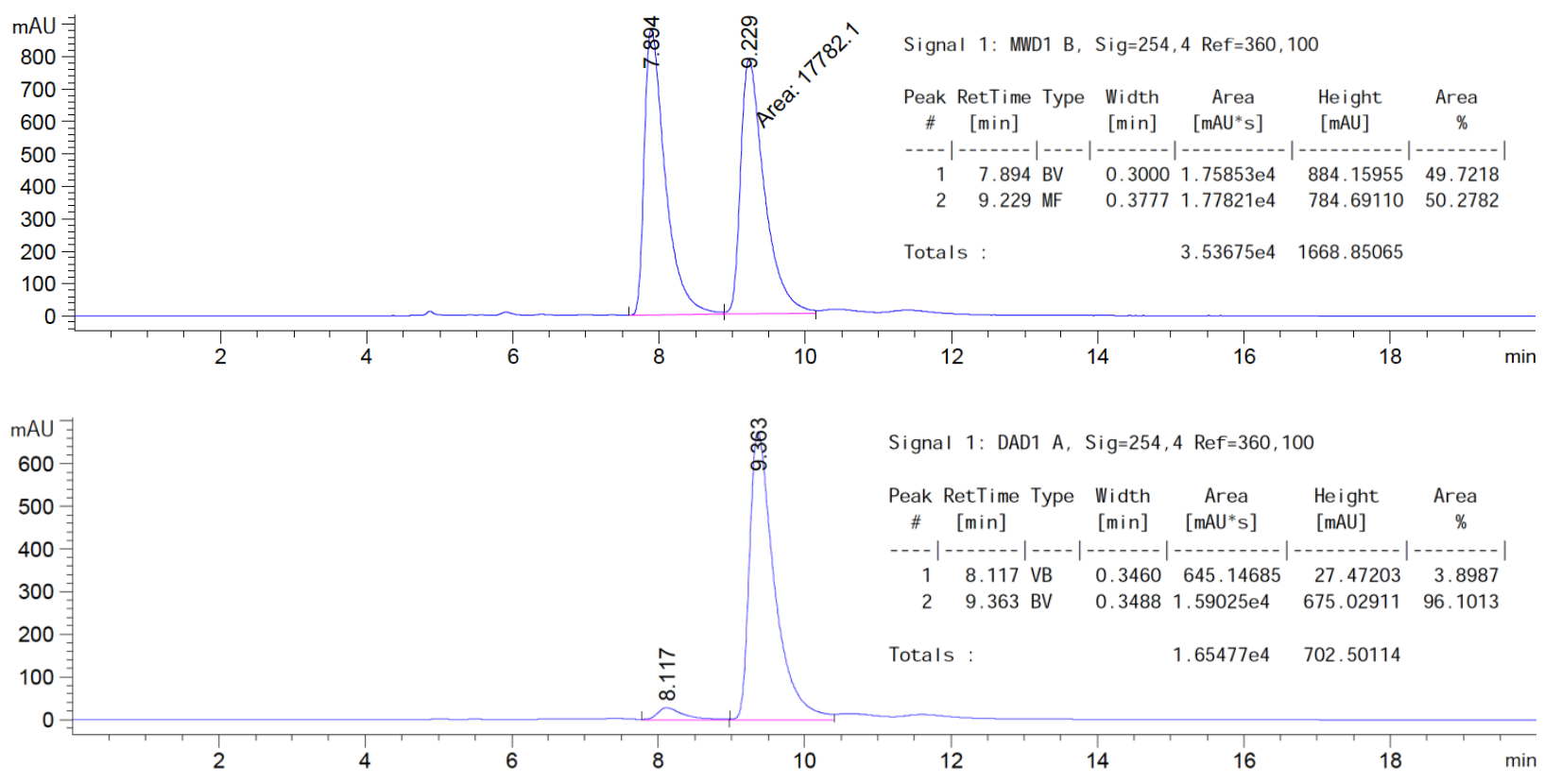


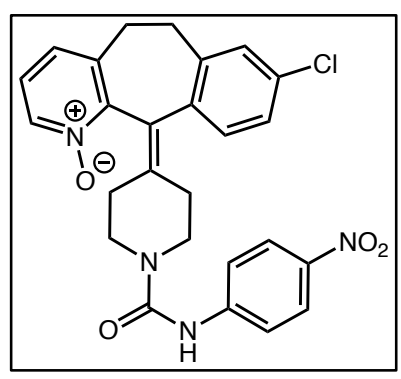

8-Chloro-11-(1-((4-nitrophenyl)carbamoyl)piperidin-4-ylidene)-6,11-dihydro$5 H$-benzo[5,6]cyclohepta-[1,2-b]pyridine 1-oxide $(6 \mathrm{~g})$

Pale-yellow solid $(13.5 \mathrm{mg}, 0.028 \mathrm{mmol}, 55 \%$ yield $)$. TLC: $\mathrm{R}_{\mathrm{f}}=0.61(10 \%$ $\mathrm{MeOH} / \mathrm{EtOAc}$ ). IR (FT-ATR, neat): 1665, 1595, 1500, 1419, 1326, 1301, 1214, $1111,751 \mathrm{~cm}^{-1} .{ }^{1} \mathrm{H}$ NMR $\left(500 \mathrm{MHz}, \mathrm{CDCl}_{3}\right) \delta 8.13-8.05(\mathrm{~m}, 2 \mathrm{H}), 7.55-7.46(\mathrm{~m}$, $3 \mathrm{H}), 7.19(\mathrm{~d}, J=8.1 \mathrm{~Hz}, 1 \mathrm{H}), 7.13(\mathrm{td}, J=12.4,11.8,5.5 \mathrm{~Hz}, 4 \mathrm{H}), 3.85(\mathrm{ddd}, J=12.2$, 7.7, $4.1 \mathrm{~Hz}, 1 \mathrm{H}), 3.74$ (ddd, $J=11.9,6.9,4.4 \mathrm{~Hz}, 1 \mathrm{H}), 3.57$ (dddd, $J=19.8,12.2,7.3$, $4.2 \mathrm{~Hz}, 2 \mathrm{H}), 3.42-3.30(\mathrm{~m}, 2 \mathrm{H}), 2.88(\mathrm{ddt}, J=24.2,15.1,4.6 \mathrm{~Hz}, 2 \mathrm{H}), 2.67$ (ddd, $J$ $=14.0,7.7,4.3 \mathrm{~Hz}, 1 \mathrm{H}), 2.42(\mathrm{tdt}, J=14.4,7.0,4.1 \mathrm{~Hz}, 2 \mathrm{H}), 2.09(\mathrm{ddd}, J=14.1,7.7$, $4.2 \mathrm{~Hz}, 1 \mathrm{H}) .{ }^{13} \mathrm{C}$ NMR $\left(126 \mathrm{MHz}, \mathrm{CDCl}_{3}\right) \delta 154.1,149.4,145.9,142.4,140.5,138.3,137.7,134.0,133.8,132.3$, 130.3, 126.5, 125.1, 124.3, 124.0, 118.6, 77.2, 44.8 (2 C), 32.0, 31.9, 30.6, 30.6. HRMS (ESI) $\mathrm{m} / z:[\mathrm{M}+\mathrm{H}]^{+}$calcd for $\mathrm{C}_{26} \mathrm{H}_{24} \mathrm{ClN}_{4} \mathrm{O}_{4}{ }^{+}$491.1481, found 491.1503. HPLC (Chiralpak IB column, $60 \% \mathrm{EtOH} /$ hexanes, $0.7 \mathrm{~mL} / \mathrm{min}$ flowrate, $2 \mu \mathrm{L}$ injection, $25^{\circ} \mathrm{C}, 254 \mathrm{~nm}$ ): minor enantiomer $t_{\mathrm{R}}=8.7 \mathrm{~min}$, major enantiomer $t_{\mathrm{R}}=10.0 \mathrm{~min}$. $[\boldsymbol{\alpha}]_{589}^{\mathbf{2 0}}$ $\left(\mathrm{c}=0.68, \mathrm{CHCl}_{3} ; 92: 8 \mathrm{er}\right):+111.6$.
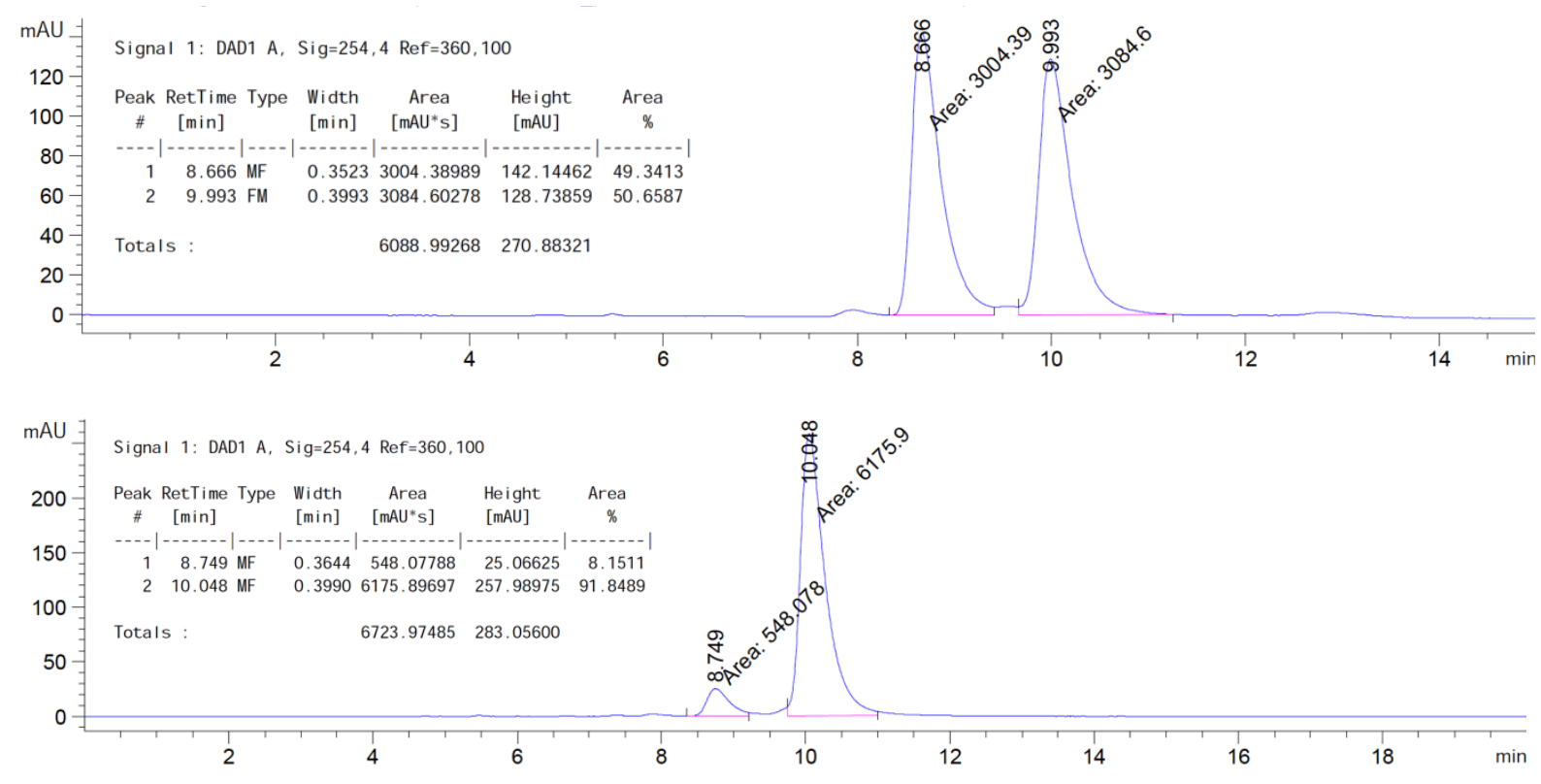
<smiles>O=C(Nc1cc(C(F)(F)F)cc(C(F)(F)F)c1)N1CCC(=C2c3ccc(Cl)cc3C=Cc3ccc([N+](=O)[O-])cc32)CC1</smiles>

11-(1-((3,5-Bis(trifluoromethyl)phenyl)carbamoyl)piperidin-4-ylidene)-8chloro-6,11-dihydro-5 $H$-benzo[5,6]cyclohepta[1,2-b]pyridine 1-oxide $(6 \mathrm{~h})$ White solid (10.8 mg, 0.019 mmol, $37 \%$ yield $)$. TLC: $\mathrm{R}_{\mathrm{f}}=0.70(10 \% \mathrm{MeOH} / \mathrm{EtOAc})$. IR (FT-ATR, neat): 1651, 1561, 1474, 1444, 1374, 1275, 1170, 1123, 993, 878, 682 $\mathrm{cm}^{-1} .{ }^{1} \mathrm{H}$ NMR $\left(600 \mathrm{MHz}, \mathrm{CDCl}_{3}\right) \delta 8.31(\mathrm{~s}, 1 \mathrm{H}), 8.12(\mathrm{~d}, J=6.4 \mathrm{~Hz}, 1 \mathrm{H}), 7.63(\mathrm{~s}$, $2 \mathrm{H}), 7.27(\mathrm{~s}, 1 \mathrm{H}), 7.23(\mathrm{~d}, J=7.6 \mathrm{~Hz}, 1 \mathrm{H}), 7.18(\mathrm{t}, J=7.1 \mathrm{~Hz}, 1 \mathrm{H}), 7.00(\mathrm{~d}, J=8.2$ $\mathrm{Hz}, 1 \mathrm{H}), 6.98(\mathrm{~s}, 1 \mathrm{H}), 6.87(\mathrm{~d}, J=8.2 \mathrm{~Hz}, 1 \mathrm{H}), 3.93(\mathrm{ddd}, J=12.1,7.5,4.1 \mathrm{~Hz}, 1 \mathrm{H})$, $3.80(\mathrm{dt}, J=11.7,4.7 \mathrm{~Hz}, 1 \mathrm{H}), 3.64-3.55(\mathrm{~m}, 2 \mathrm{H}), 3.43-3.28(\mathrm{~m}, 2 \mathrm{H}), 2.91-2.80$ $(\mathrm{m}, 2 \mathrm{H}), 2.68-2.61(\mathrm{~m}, 1 \mathrm{H}), 2.54(\mathrm{ddd}, J=13.8,6.7,4.2 \mathrm{~Hz}, 1 \mathrm{H}), 2.45(\mathrm{ddd}, J=14.2,6.5,3.9 \mathrm{~Hz}, 1 \mathrm{H}), 2.11(\mathrm{dq}$, $J=13.6,4.1 \mathrm{~Hz}, 1 \mathrm{H}) .{ }^{13} \mathrm{C}$ NMR $\left(151 \mathrm{MHz}, \mathrm{CDCl}_{3}\right) \delta 154.8,149.9,141.8,140.6,138.8,137.9,137.6,133.8,132.9$, $132.3,131.5$ (q, $J=33.0 \mathrm{~Hz}), 130.3,126.6,126.3,124.5,123.8,123.3(\mathrm{q}, J=272.8 \mathrm{~Hz}), 120.6,118.9-118.5(\mathrm{~m})$, $115.1(\mathrm{p}, J=3.8 \mathrm{~Hz}), 44.9,44.28,32.1,32.0,30.7,30.4 .{ }^{19} \mathrm{~F}$ NMR $\left(471 \mathrm{MHz}, \mathrm{CDCl}_{3}\right) \delta-63.08$. HRMS (ESI) $\mathrm{m} / z$ : $[\mathrm{M}+\mathrm{H}]^{+}$calcd for $\mathrm{C}_{28} \mathrm{H}_{23} \mathrm{ClF}_{6} \mathrm{~N}_{3} \mathrm{O}_{2}{ }^{+} 582.1378$, found 582.1389. HPLC (Chiralpak IB column, 10\% EtOH/hexanes, $0.7 \mathrm{~mL} / \mathrm{min}$ flowrate, $2 \mu \mathrm{L}$ injection, $25^{\circ} \mathrm{C}, 254 \mathrm{~nm}$ ): minor enantiomer $t_{\mathrm{R}}=14.4 \mathrm{~min}$, major enantiomer $t_{\mathrm{R}}$ $=16.5 \mathrm{~min} .[\alpha]_{\mathbf{5 8 9}}^{\mathbf{2 0}}\left(\mathrm{c}=0.41, \mathrm{CHCl}_{3} ;>99: 1 \mathrm{er}\right):+90.7$.
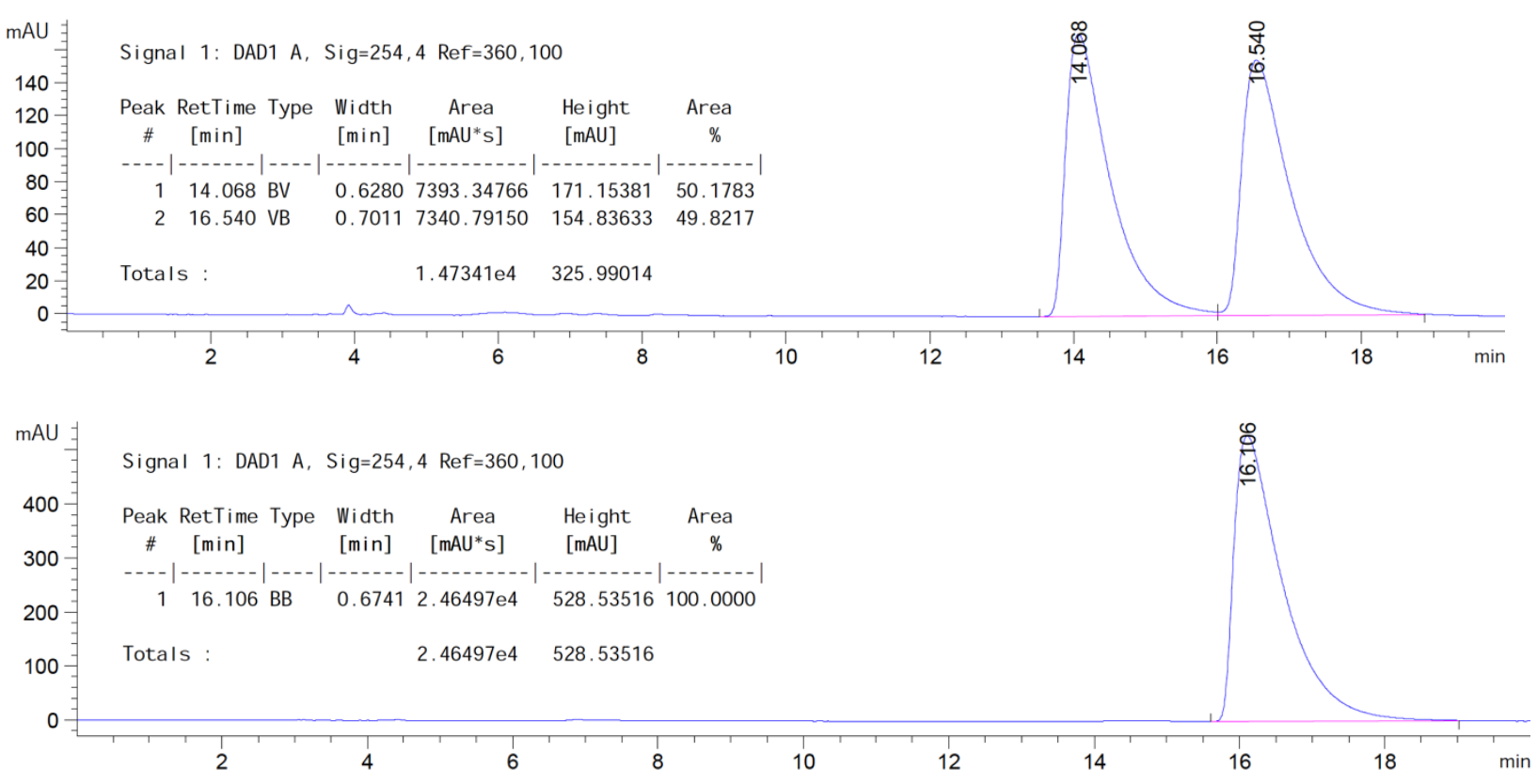

with P4 for biological testing; $[\boldsymbol{\alpha}]_{\mathbf{5 8 9}}^{\mathbf{2 0}}\left(\mathrm{c}=0.59, \mathrm{CHCl}_{3} ; 96: 4 \mathrm{er}\right):-266.1$

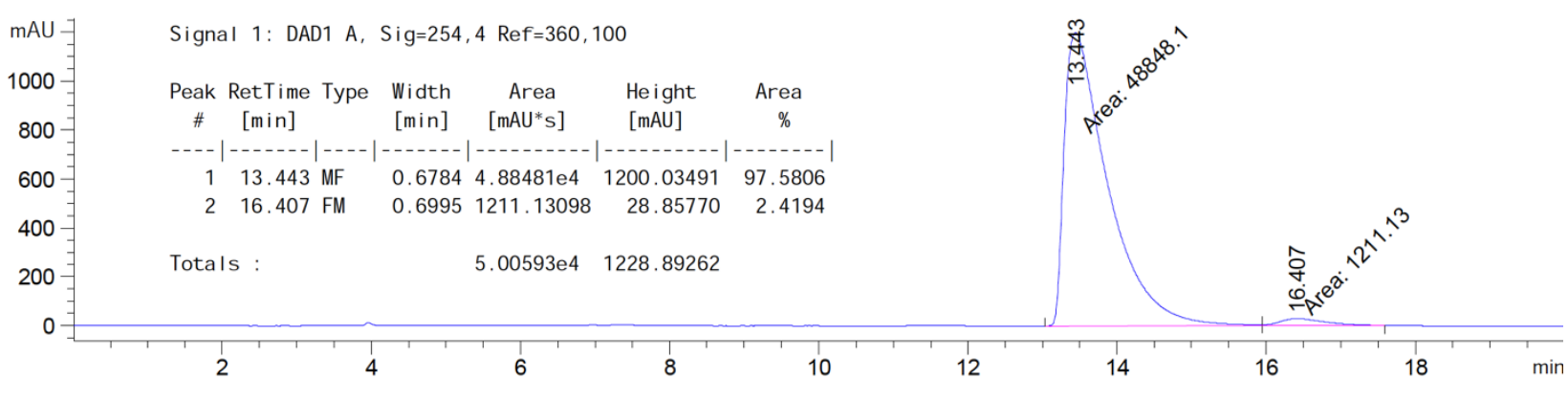




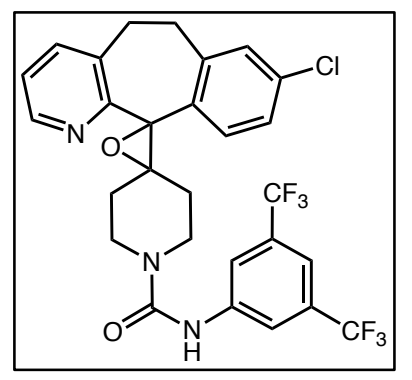

$N$-(3,5-bis(trifluoromethyl)phenyl)-8-chloro-5,6-dihydrodispiro[benzo[5,6]cyclohepta[1,2-b]pyridine-11,2'-oxirane-3',4"-piperdine]-1"-carboxamide (7h)

White solid (11.2 mg, 0.019 mmol, $38 \%$ yield). TLC: $\mathrm{R}_{f}=0.84(10 \% \mathrm{MeOH} / \mathrm{EtOAc})$. IR (FT-ATR, neat): 1651, 1562, 1474, 1375, 1276, 1172, 1128, 1002, $683 \mathrm{~cm}^{-1} .{ }^{1} \mathrm{H}$ $\operatorname{NMR}\left(600 \mathrm{MHz}, \mathrm{CD}_{3} \mathrm{OD}\right) \delta 8.37-8.35(\mathrm{~m}, 1 \mathrm{H}), 8.04(\mathrm{~d}, J=1.4 \mathrm{~Hz}, 2 \mathrm{H}), 7.70(\mathrm{~d}, J$ $=7.1 \mathrm{~Hz}, 1 \mathrm{H}), 7.51(\mathrm{~s}, 1 \mathrm{H}), 7.47(\mathrm{~d}, J=8.3 \mathrm{~Hz}, 1 \mathrm{H}), 7.33(\mathrm{dd}, J=7.7,4.9 \mathrm{~Hz}, 1 \mathrm{H})$, $7.26(\mathrm{~d}, J=1.8 \mathrm{~Hz}, 1 \mathrm{H}), 7.24(\mathrm{dd}, J=8.3,2.0 \mathrm{~Hz}, 1 \mathrm{H}), 4.12(\mathrm{t}, J=15.0 \mathrm{~Hz}, 2 \mathrm{H}), 3.40$ $(\mathrm{ddt}, J=17.5,14.7,5.6 \mathrm{~Hz}, 2 \mathrm{H}), 3.34-3.25(\mathrm{~m}, 2 \mathrm{H}), 3.07-2.93(\mathrm{~m}, 2 \mathrm{H}), 1.92-1.78$ $(\mathrm{m}, 2 \mathrm{H}), 1.23-1.17(\mathrm{~m}, 1 \mathrm{H}), 1.09-1.04(\mathrm{~m}, 1 \mathrm{H}) .{ }^{13} \mathrm{C} \mathrm{NMR}\left(151 \mathrm{MHz}, \mathrm{CD}_{3} \mathrm{OD}\right) \delta$ 156.6, 155.6, 147.4, 143.6, $141.1,139.7,135.9,135.6,135.1,132.9$ (q, $J=33.0 \mathrm{~Hz}), 130.3,129.1,127.3,124.9,124.9$ (q, $J=271.7 \mathrm{~Hz}), 120.7$ - 120.5 (m), 116.14 - 115.95 (m), 70.3, 69.6, 43.7, 43.6, 32.4, 31.6, 30.6, 30.5. ${ }^{19} \mathrm{~F}$ NMR $\left(471 \mathrm{MHz}, \mathrm{CD}_{3} \mathrm{OD}\right) \delta$ 63.03. HRMS (ESI) $\mathrm{m} / \mathrm{z}:[\mathrm{M}+\mathrm{H}]^{+}$calcd for $\mathrm{C}_{28} \mathrm{H}_{23} \mathrm{ClF}_{6} \mathrm{~N}_{3} \mathrm{O}_{2}{ }^{+}$582.1378, found 582.1385. HPLC (Chiralpak IB column, $5 \% \mathrm{EtOH} /$ hexanes, $1.0 \mathrm{~mL} / \mathrm{min}$ flowrate, $2 \mu \mathrm{L}$ injection, $25^{\circ} \mathrm{C}, 254 \mathrm{~nm}$ ): minor enantiomer $t_{\mathrm{R}}=12.5 \mathrm{~min}$, major enantiomer $t_{\mathrm{R}}=13.8 \mathrm{~min}$.

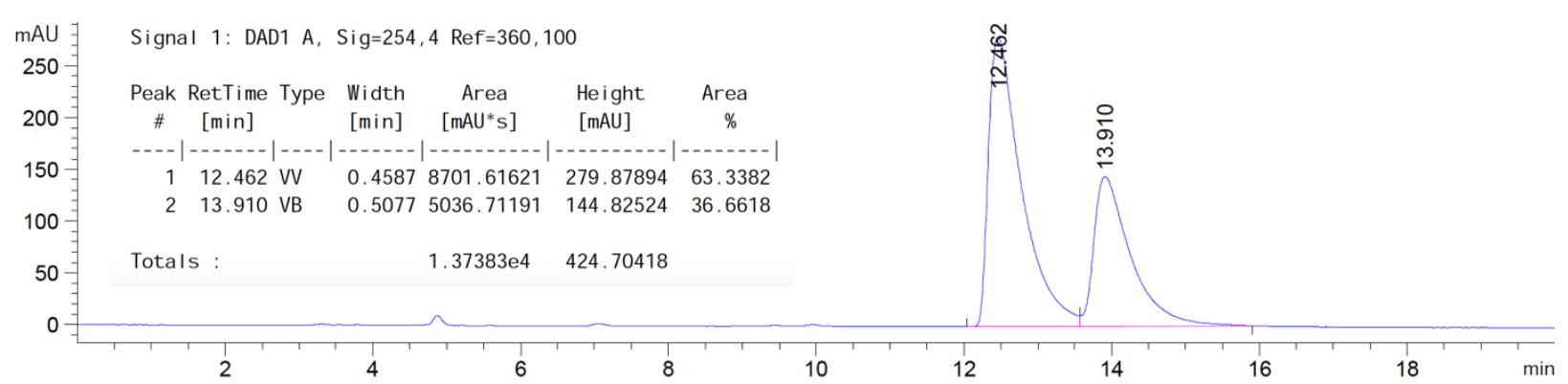

with P4
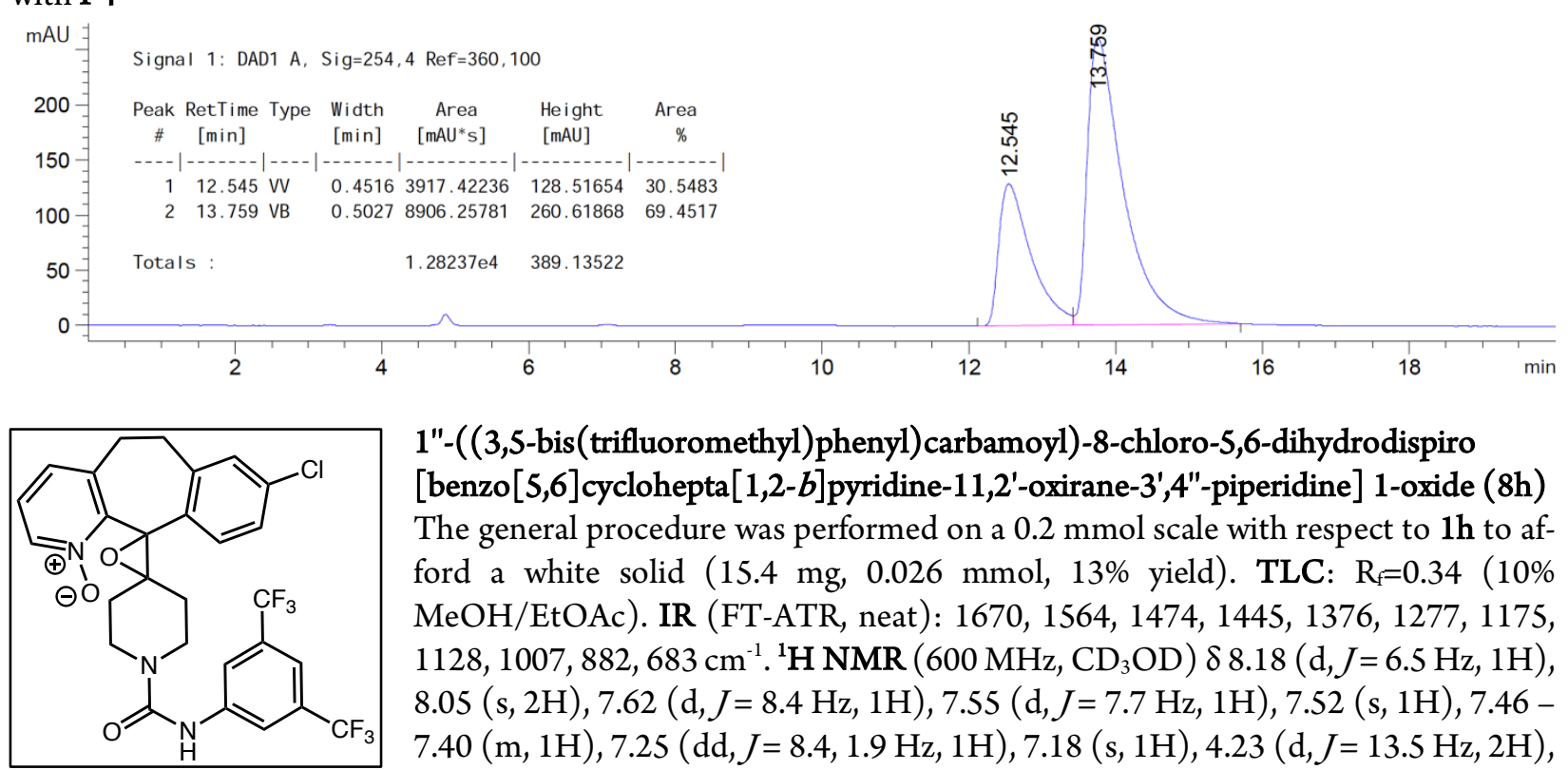

1"-((3,5-bis(trifluoromethyl)phenyl)carbamoyl)-8-chloro-5,6-dihydrodispiro [benzo[5,6]cyclohepta[1,2-b]pyridine-11,2'-oxirane-3',4"-piperidine] 1-oxide (8h) The general procedure was performed on a $0.2 \mathrm{mmol}$ scale with respect to $1 \mathrm{~h}$ to afford a white solid ( $15.4 \mathrm{mg}, 0.026 \mathrm{mmol}, 13 \%$ yield). TLC: $\mathrm{R}_{\mathrm{f}}=0.34(10 \%$ $\mathrm{MeOH} / \mathrm{EtOAc}$ ). IR (FT-ATR, neat): 1670, 1564, 1474, 1445, 1376, 1277, 1175, $1128,1007,882,683 \mathrm{~cm}^{-1} .{ }^{1} \mathrm{H}$ NMR $\left(600 \mathrm{MHz}, \mathrm{CD}_{3} \mathrm{OD}\right) \delta 8.18(\mathrm{~d}, J=6.5 \mathrm{~Hz}, 1 \mathrm{H})$, $8.05(\mathrm{~s}, 2 \mathrm{H}), 7.62(\mathrm{~d}, J=8.4 \mathrm{~Hz}, 1 \mathrm{H}), 7.55(\mathrm{~d}, J=7.7 \mathrm{~Hz}, 1 \mathrm{H}), 7.52(\mathrm{~s}, 1 \mathrm{H}), 7.46-$ $7.40(\mathrm{~m}, 1 \mathrm{H}), 7.25(\mathrm{dd}, J=8.4,1.9 \mathrm{~Hz}, 1 \mathrm{H}), 7.18(\mathrm{~s}, 1 \mathrm{H}), 4.23(\mathrm{~d}, J=13.5 \mathrm{~Hz}, 2 \mathrm{H})$, $3.43(\mathrm{dt}, J=18.0,3.6 \mathrm{~Hz}, 1 \mathrm{H}), 3.36-3.28(\mathrm{~m}, 1 \mathrm{H}), 3.28-3.19(\mathrm{~m}, 2 \mathrm{H}), 3.06(\mathrm{dt}, J$ $=14.5,3.9 \mathrm{~Hz}, 1 \mathrm{H}), 2.95(\mathrm{ddd}, J=18.3,14.3,4.4 \mathrm{~Hz}, 1 \mathrm{H}), 2.00(\mathrm{dtd}, J=30.6,13.0,4.9 \mathrm{~Hz}, 2 \mathrm{H}), 1.15(\mathrm{~d}, J=14.0$ $\mathrm{Hz}, 1 \mathrm{H}), 0.99-0.77(\mathrm{~m}, 1 \mathrm{H}) .{ }^{13} \mathrm{C}$ NMR $\left(151 \mathrm{MHz}, \mathrm{CD}_{3} \mathrm{OD}\right) \delta 156.6,148.0,143.6,140.8,140.4,139.4,135.6$, $132.9(\mathrm{q}, J=33.0 \mathrm{~Hz}), 131.5,131.3,130.0,129.9,127.1,127.0,124.9(\mathrm{q}, J=272.0 \mathrm{~Hz}), 121.5-119.8(\mathrm{~m}), 116.15$ - 116.0 (m), 69.4, 66.2, 43.7, 43.6, 32.9, 32.1, 30.2, 30.1. ${ }^{19} \mathrm{~F}$ NMR (471 MHz, CD ${ }_{3} \mathrm{OD}$ ) $\delta-63.28$. HRMS (ESI) $\mathrm{m} / z:[\mathrm{M}+\mathrm{H}]^{+}$calcd for $\mathrm{C}_{28} \mathrm{H}_{23} \mathrm{ClF}_{6} \mathrm{~N}_{3} \mathrm{O}_{3}{ }^{+} 598.1327$, found 598.1321. X-ray characterization: recrystallized from methanol. 


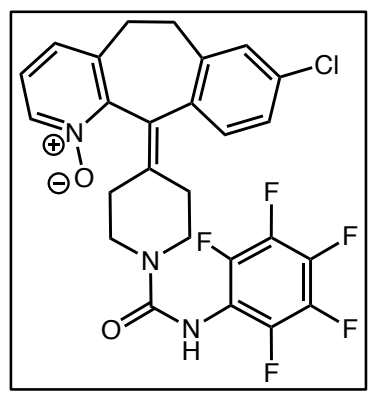

8-Chloro-11-(1-((perfluorophenyl)carbamoyl)piperidin-4-ylidene)-6,11-dihydro-5 $H$-benzo $[5,6]$ cyclohepta $[1,2-b]$ pyridine 1-oxide (6i)

White solid (11.8 mg, $0.022 \mathrm{mmol}, 44 \%$ yield). TLC: $\mathrm{R}_{\mathrm{f}}=0.47$ (10\% MeOH/EtOAc). IR (FT-ATR, neat): 1645, 1523, 1495, 1466, 1253, 1242, 992, $748 \mathrm{~cm}^{-1} .{ }^{1} \mathrm{H}$ NMR $(600$ $\left.\mathrm{MHz}, \mathrm{CD}_{3} \mathrm{OD}\right) \delta 8.20(\mathrm{~d}, J=6.4 \mathrm{~Hz}, 1 \mathrm{H}), 7.50(\mathrm{~d}, J=7.7 \mathrm{~Hz}, 1 \mathrm{H}), 7.38(\mathrm{dd}, J=7.7,6.5$ $\mathrm{Hz}, 1 \mathrm{H}), 7.36-7.31(\mathrm{~m}, 1 \mathrm{H}), 7.20(\mathrm{dd}, J=5.8,2.2 \mathrm{~Hz}, 2 \mathrm{H}), 3.83$ (ddt, $J=17.7,11.2$, $4.7 \mathrm{~Hz}, 2 \mathrm{H}), 3.55-3.37(\mathrm{~m}, 5 \mathrm{H}), 3.01-2.88(\mathrm{~m}, 2 \mathrm{H}), 2.67-2.58(\mathrm{~m}, 1 \mathrm{H}), 2.44$ (ddd, $J=14.3,6.4,4.0 \mathrm{~Hz}, 1 \mathrm{H}), 2.36(\mathrm{ddt}, J=12.7,8.3,4.3 \mathrm{~Hz}, 1 \mathrm{H}), 2.07$ (ddd, $J=14.3,6.5$, $4.0 \mathrm{~Hz}, 1 \mathrm{H}) .{ }^{13} \mathrm{C}$ NMR $\left(151 \mathrm{MHz}, \mathrm{CD}_{3} \mathrm{OD}\right) \delta 156.9,150.7,146.2-143.9(\mathrm{~m}), 142.5$, 141.8 - $141.3(\mathrm{~m}), 140.5,140.4,140.0$ - 137.9 (m), 138.5, 134.9, 134.9, 133.6, 131.3, 130.2, 127.1, 126.2, 124.7, 116.3 - $115.6(\mathrm{~m}), 46.0,45.6,32.8,32.7,31.2,31.1 .{ }^{19} \mathrm{~F}$ NMR $\left(471 \mathrm{MHz}, \mathrm{CDCl}_{3}\right) \delta-147.39(\mathrm{~d}, J=17.0 \mathrm{~Hz})$, $159.23(\mathrm{t}, J=21.6 \mathrm{~Hz}),-163.31\left(\mathrm{td}, J=22.5,5.8 \mathrm{~Hz}\right.$ ). HRMS (ESI) $\mathrm{m} / z:[\mathrm{M}+\mathrm{H}]^{+}$calcd for $\mathrm{C}_{26} \mathrm{H}_{20} \mathrm{ClF}_{5} \mathrm{~N}_{3} \mathrm{O}_{2}{ }^{+}$ 536.1159, found 536.1166. HPLC (Chiralpak IB column, $60 \% \mathrm{EtOH} /$ hexanes, $0.7 \mathrm{~mL} / \mathrm{min}$ flowrate, $2 \mu \mathrm{L}$ injection, $\left.25^{\circ} \mathrm{C}, 254 \mathrm{~nm}\right)$ : minor enantiomer $t_{\mathrm{R}}=6.7 \mathrm{~min}$, major enantiomer $t_{\mathrm{R}}=7.4 \mathrm{~min}$. $[\alpha]_{589}^{20}\left(\mathrm{c}=0.26, \mathrm{CHCl}_{3}\right.$; 94:6 er): + 93.0.
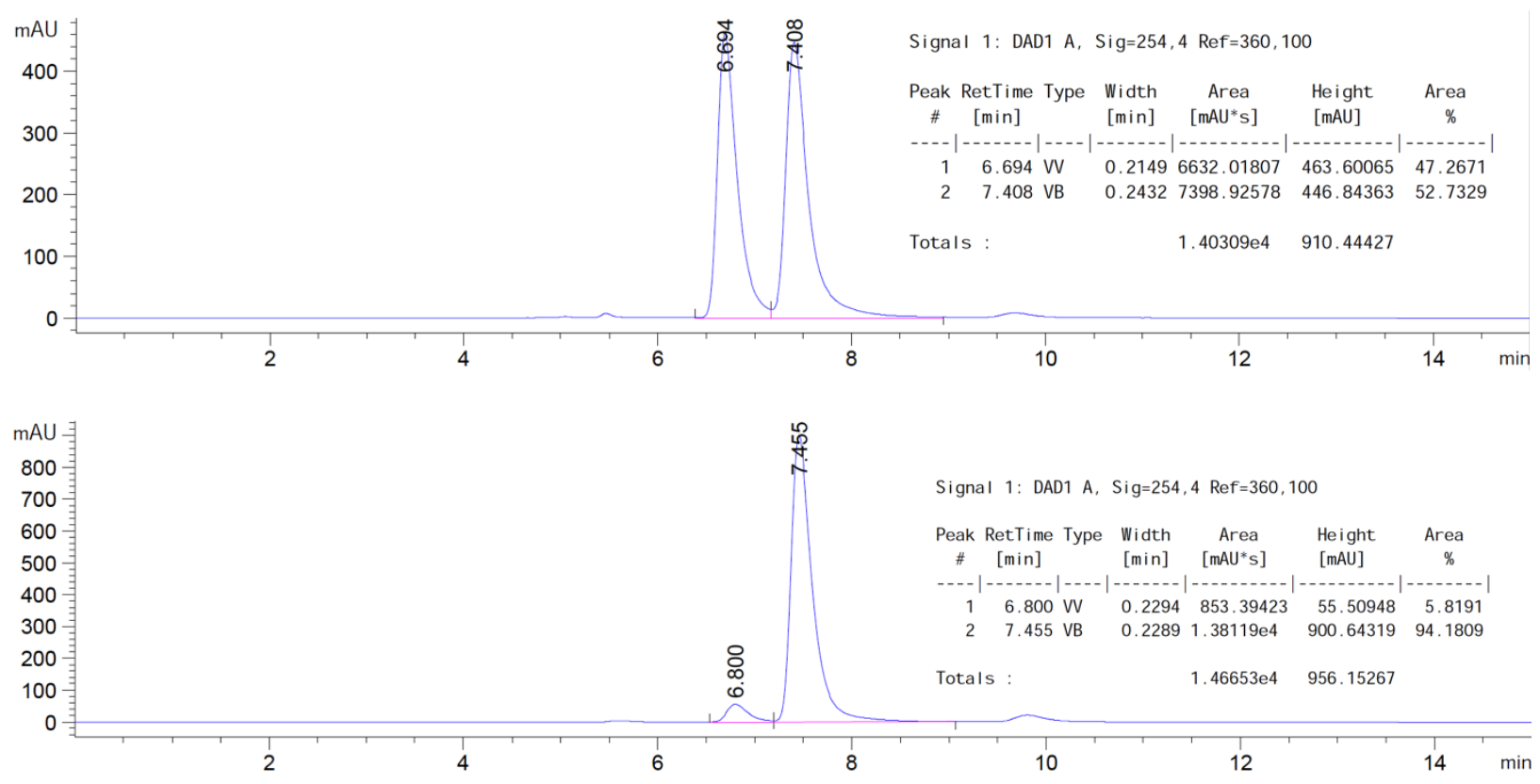


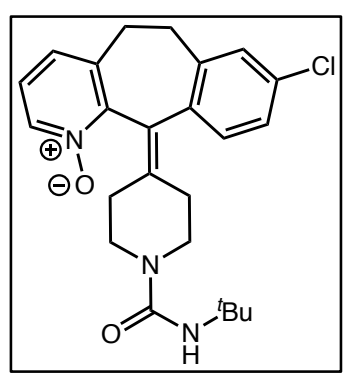

11-(1-( $t$-Butylcarbamoyl)piperidin-4-ylidene)-8-chloro-6,11-dihydro-5 $H$-benzo$[5,6]$ cyclohepta $[1,2-b]$ pyridine 1-oxide (6j)

White solid ( $8.1 \mathrm{mg}, 0.019 \mathrm{mmol}, 38 \%$ yield). TLC: $\mathrm{R}_{\mathrm{f}}=0.60$ (10\% MeOH/EtOAc). IR (FT-ATR, neat): 1627, 1531, 1478, 1392, 1255, 1209, $990 \mathrm{~cm}^{-1} .{ }^{1} \mathrm{H}$ NMR $(600 \mathrm{MHz}$, $\left.\mathrm{CD}_{3} \mathrm{OD}\right) \delta 8.18(\mathrm{~d}, J=6.4 \mathrm{~Hz}, 1 \mathrm{H}), 7.48(\mathrm{~d}, J=7.7 \mathrm{~Hz}, 1 \mathrm{H}), 7.36(\mathrm{dd}, J=7.6,6.6 \mathrm{~Hz}$, $1 \mathrm{H}), 7.31-7.27(\mathrm{~m}, 1 \mathrm{H}), 7.17(\mathrm{~d}, J=6.5 \mathrm{~Hz}, 2 \mathrm{H}), 3.70-3.59(\mathrm{~m}, 2 \mathrm{H}), 3.46-3.34(\mathrm{~m}$, $2 \mathrm{H}), 3.22$ (dddd, $J=18.7,12.8,8.8,3.9 \mathrm{~Hz}, 2 \mathrm{H}), 2.98-2.86(\mathrm{~m}, 2 \mathrm{H}), 2.50$ (ddd, $J=13.6$, 8.8, $4.4 \mathrm{~Hz}, 1 \mathrm{H}), 2.34$ (ddd, $J=14.2,6.1,3.9 \mathrm{~Hz}, 1 \mathrm{H}), 2.26(\mathrm{ddd}, J=13.6,8.6,4.4 \mathrm{~Hz}$, $1 \mathrm{H}), 1.94(\mathrm{ddd}, J=14.1,6.4,4.0 \mathrm{~Hz}, 2 \mathrm{H}), 1.32(\mathrm{~s}, 9 \mathrm{H}) .{ }^{13} \mathrm{C} \mathrm{NMR}\left(151 \mathrm{MHz}, \mathrm{CD}_{3} \mathrm{OD}\right) \delta 159.3,150.9,143.5,140.5$, 140.3, 138.5, 135.0, 134.8, 133.6, 131.3, 130.2, 127.0, 126.1, 124.0, 51.7, 45.7, 45.3, 32.9, 32.8, 31.4, 31.1, 29.7. HRMS (ESI) $\mathrm{m} / \mathrm{z}:[\mathrm{M}+\mathrm{H}]^{+}$calcd for $\mathrm{C}_{24} \mathrm{H}_{29} \mathrm{ClN}_{3} \mathrm{O}_{2}{ }^{+} 426.1943$, found 426.1947. HPLC (Chiralpak IB column, $50 \% \mathrm{EtOH} /$ hexanes, $0.7 \mathrm{~mL} / \mathrm{min}$ flowrate, $2 \mu \mathrm{L}$ injection, $25^{\circ} \mathrm{C}, 254 \mathrm{~nm}$ ): minor enantiomer $t_{\mathrm{R}}=6.4$ min, major enantiomer $t_{\mathrm{R}}=6.9 \mathrm{~min} .[\alpha]_{589}^{20}\left(\mathrm{c}=0.45, \mathrm{CHCl}_{3} ; 82: 18 \mathrm{er}\right):+75.4$.
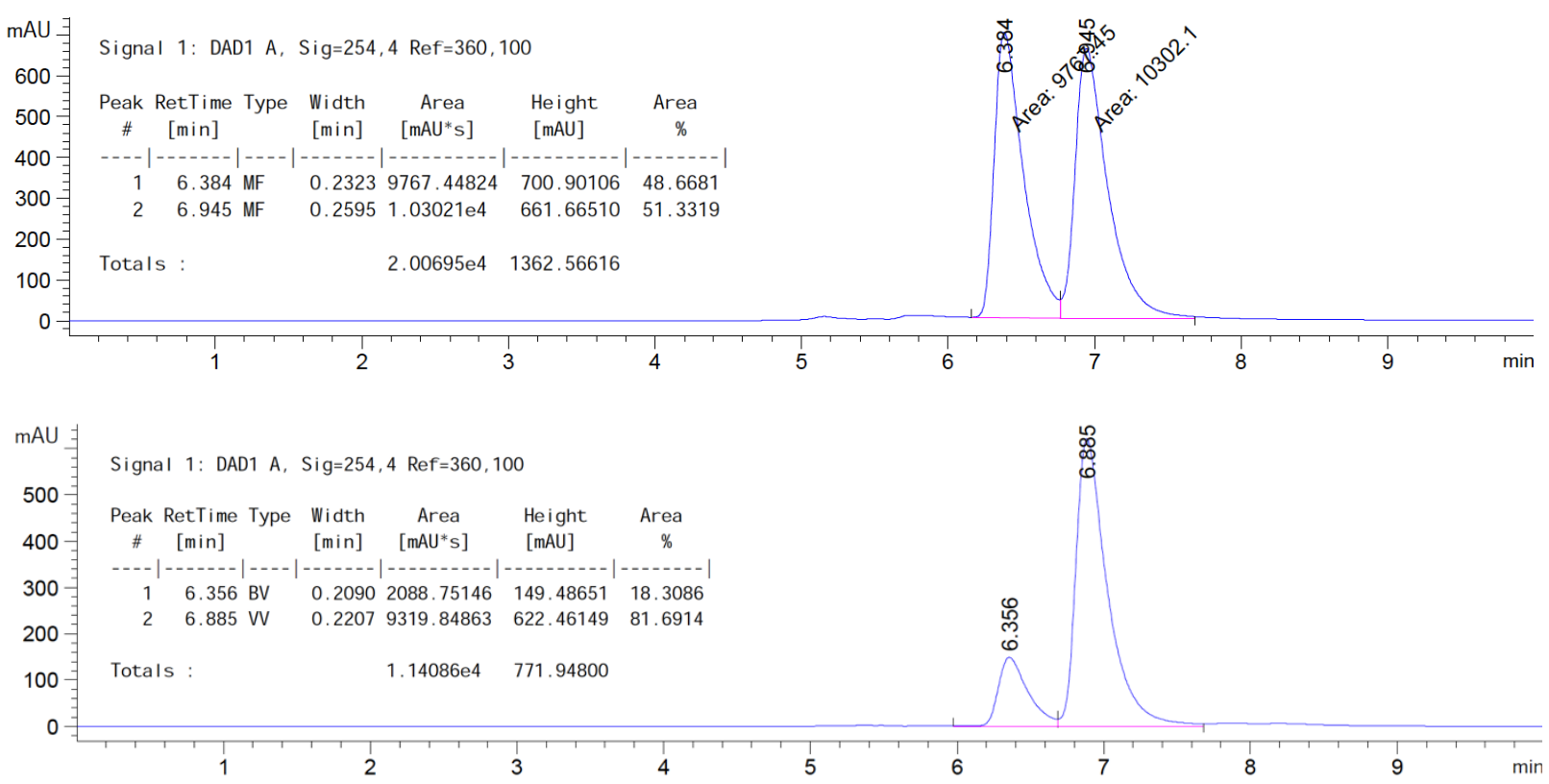


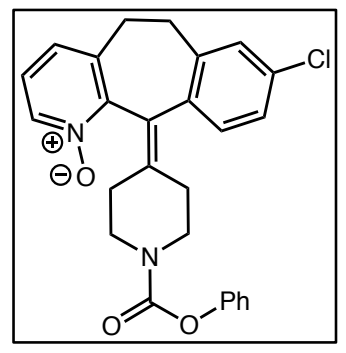

8-Chloro-11-(1-(phenoxycarbonyl)piperidin-4-ylidene)-6,11-dihydro-5 $H$-benzo$[5,6]$ cyclohepta $[1,2-b]$ pyridine 1 -oxide $(6 \mathrm{k})$

A second column (SNAP Ultra $\mathrm{C} 1830 \mathrm{~g}, 0-85 \% \mathrm{MeCN} / \mathrm{H}_{2} \mathrm{O}$ for $5 \mathrm{CV}$, then $85 \%$ $\mathrm{MeCN} / \mathrm{H}_{2} \mathrm{O}$ for $2 \mathrm{CV}$, then $85-100 \% \mathrm{MeCN} / \mathrm{H}_{2} \mathrm{O}$ for $2 \mathrm{CV}$ ) was required to obtain a pure white solid $9.8 \mathrm{mg}, 0.22 \mathrm{mmol}, 44 \%$ yield). TLC: $\mathrm{R}_{\mathrm{f}}=0.71(10 \% \mathrm{MeOH} / \mathrm{EtOAc})$. IR (FT-ATR, neat): 1710, 1421, 1197, 993, $745 \mathrm{~cm}^{-1} .{ }^{1} \mathrm{H}$ NMR $\left(600 \mathrm{MHz}, \mathrm{CD}_{3} \mathrm{OD}\right) \delta$ $8.20(\mathrm{~d}, J=6.4 \mathrm{~Hz}, 1 \mathrm{H}), 7.50(\mathrm{~d}, J=7.8 \mathrm{~Hz}, 1 \mathrm{H}), 7.37(\mathrm{t}, J=7.8 \mathrm{~Hz}, 3 \mathrm{H}), 7.35-7.31(\mathrm{~m}$, $1 \mathrm{H}), 7.22(\mathrm{~d}, J=7.4 \mathrm{~Hz}, 1 \mathrm{H}), 7.21-7.18(\mathrm{~m}, 2 \mathrm{H}), 7.10(\mathrm{~d}, J=7.9 \mathrm{~Hz}, 2 \mathrm{H}), 4.00-3.91$ $(\mathrm{m}, 1 \mathrm{H}), 3.86-3.76(\mathrm{~m}, 1 \mathrm{H}), 3.66-3.54(\mathrm{~m}, 1 \mathrm{H}), 3.50-3.37(\mathrm{~m}, 3 \mathrm{H}), 3.00-2.87(\mathrm{~m}, 2 \mathrm{H}), 2.68-2.56(\mathrm{~m}, 1 \mathrm{H})$, $2.51-2.40(\mathrm{~m}, 1 \mathrm{H}), 2.40-2.29(\mathrm{~m}, 1 \mathrm{H}), 2.12-2.02(\mathrm{~m}, 1 \mathrm{H}) .{ }^{13} \mathrm{C}$ NMR $\left(151 \mathrm{MHz}, \mathrm{CD}_{3} \mathrm{OD}\right) \delta 155.5,152.8$, 150.7, 142.4, 140.5, 140.4, 138.5, 134.9, 133.6, 131.3, 130.4, 130.2, 130.2, 127.1, 126.6, 126.2, 124.8, 122.9, 46.0 (d, $J=49.4 \mathrm{~Hz}$ ), $45.6(\mathrm{~d}, J=55.2 \mathrm{~Hz}), 32.8,32.8(\mathrm{~d}, J=55.2 \mathrm{~Hz}), 31.4,31.1$. HRMS (ESI) $\mathrm{m} / \mathrm{z}:[\mathrm{M}+\mathrm{H}]^{+}$calcd for $\mathrm{C}_{26} \mathrm{H}_{24} \mathrm{ClN}_{2} \mathrm{O}_{3}{ }^{+}$447.1470, found 447.1483. HPLC (Chiralpak IC column, 80\% $\mathrm{EtOH} /$ hexanes, $0.7 \mathrm{~mL} / \mathrm{min}$ flowrate, $2 \mu \mathrm{L}$ injection, $25^{\circ} \mathrm{C}, 254 \mathrm{~nm}$ ): minor enantiomer $t_{\mathrm{R}}=16.2 \mathrm{~min}$, major enantiomer $t_{\mathrm{R}}=22.0 \mathrm{~min}$. $[\boldsymbol{\alpha}]_{589}^{\mathbf{2 0}}$ $\left(\mathrm{c}=0.33, \mathrm{CHCl}_{3} ; 65: 35\right.$ er $):+36.0$.
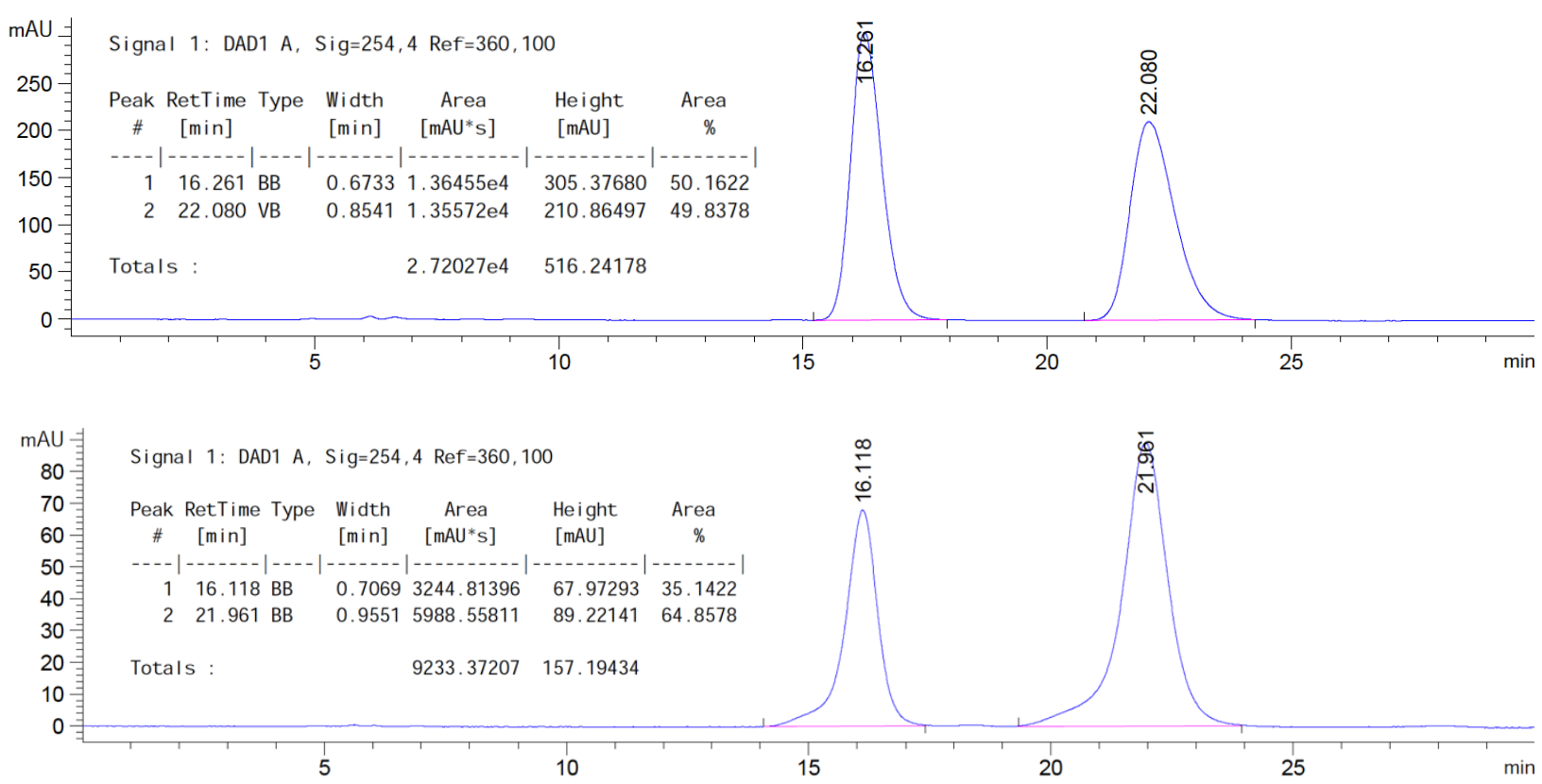


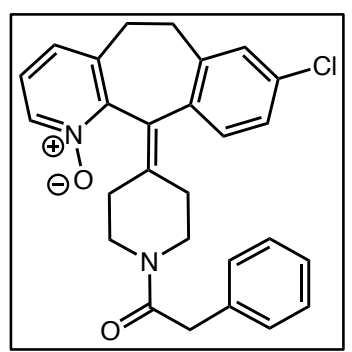

8-Chloro-11-(1-(2-phenylacetyl)piperidin-4-ylidene)-6,11-dihydro-5Hbenzo $[5,6]$ cyclohepta $[1,2-b]$ pyridine 1-oxide $(61)$

White solid (5.8 mg, $0.013 \mathrm{mmol}, 26 \%$ yield). TLC: $\mathrm{R}_{\mathrm{f}}=0.21$ (10\% MeOH/EtOAc). IR (FT-ATR, neat): 1625, 1456, 1260, 1090, 1027, 993, $798 \mathrm{~cm}^{-1} .{ }^{1} \mathrm{H}$ NMR $(600 \mathrm{MHz}$, $\left.\mathrm{CD}_{3} \mathrm{OD}\right) \delta 8.16(\mathrm{ddd}, J=16.6,6.5,1.1 \mathrm{~Hz}, 1 \mathrm{H}), 7.47(\mathrm{dd}, J=7.3,5.3 \mathrm{~Hz}, 1 \mathrm{H}), 7.38-$ $7.33(\mathrm{~m}, 1 \mathrm{H}), 7.33-7.21(\mathrm{~m}, 6 \mathrm{H}), 7.19-7.13(\mathrm{~m}, 2 \mathrm{H}), 3.95-3.85(\mathrm{~m}, 1 \mathrm{H}), 3.81(\mathrm{~s}$, $2 \mathrm{H}), 3.77(\mathrm{ddt}, J=13.9,7.1,4.0 \mathrm{~Hz}, 1 \mathrm{H}), 3.48(\mathrm{ddtt}, J=25.3,12.6,8.4,4.2 \mathrm{~Hz}, 2 \mathrm{H}), 3.37$ (dddd, $J=16.8,12.9,8.2,3.8 \mathrm{~Hz}, 2 \mathrm{H}), 2.99-2.81(\mathrm{~m}, 2 \mathrm{H}), 2.50(\mathrm{ddd}, J=13.2,8.5,4.5$ $\left.\mathrm{Hz}, 0.5 \mathrm{H}^{*}\right), 2.36-2.30(\mathrm{~m}, 1 \mathrm{H}), 2.23\left(\mathrm{ddd}, J=13.3,8.6,4.5 \mathrm{~Hz}, 0.5 \mathrm{H}^{*}\right), 2.17$ (ddd, $J=14.3,6.8,4.0 \mathrm{~Hz}, 0.5 \mathrm{H}^{*}$ ), 2.09 (ddd, $J=14.1,8.1,4.3 \mathrm{~Hz}, 0.5 \mathrm{H}^{*}$ ), 1.95 (ddd, $J=14.2,6.4,4.1 \mathrm{~Hz}, 0.5 \mathrm{H}^{*}$ ), 1.79 (ddd, $J=14.3,6.8,4.0 \mathrm{~Hz}$, $\left.0.5 \mathrm{H}^{*}\right) .{ }^{13} \mathrm{C}$ NMR $\left(151 \mathrm{MHz}, \mathrm{CD}_{3} \mathrm{OD}\right) \delta 172.2 / 172.2$ (1C), 150.6, 142.3, 140.4, 140.3/140.3 (1C), 138.5/138.4 (1C), 136.5, 134.9/134.9 (1C), 134.8/134.8 (1C), 133.5/133.4 (1C), 131.3/131.3 (1C), 130.2, 129.8, 129.7/129.7 (1C), 127.9, 127.0/127.0 (1C), 126.2/126.2 (1C), 124.7/124.7 (1C), 47.6/47.3 (1C), 43.8/43.3 (1C), 41.6/41.6 (1C), 33.0/32.4 (1C), 32.78, 31.5/30.9 (1C), 31.1/31.1 (1C) [ ${ }^{*}$ Note: two conformations were detected via ${ }^{1} \mathrm{H}$ and ${ }^{13} \mathrm{C}$ NMR]. HRMS (ESI) $\mathrm{m} / z$ : $[\mathrm{M}+\mathrm{H}]^{+}$calcd for $\mathrm{C}_{27} \mathrm{H}_{26} \mathrm{ClN}_{2} \mathrm{O}_{2}{ }^{+}$445.1677, found 445.1693. HPLC (Chiralpak IB column, $60 \% \mathrm{EtOH} /$ hexanes, $0.7 \mathrm{~mL} / \mathrm{min}$ flowrate, $2 \mu \mathrm{L}$ injection, $25^{\circ} \mathrm{C}, 254 \mathrm{~nm}$ ): minor enantiomer $t_{\mathrm{R}}=9.3 \mathrm{~min}$, major enantiomer $t_{\mathrm{R}}=12.4 \mathrm{~min}$. $[\boldsymbol{\alpha}]_{589}^{20}\left(\mathrm{c}=0.30, \mathrm{CHCl}_{3} ; 71: 29 \mathrm{er}\right):+44.6$.

The racemic standard was prepared by dissolving 11 ( $12.9 \mathrm{mg}, 0.03 \mathrm{mmol}, 1.00$ equiv) and Boc-L/D-Asp-OMe (0.7 $\mathrm{mg}, 10 \mathrm{~mol} \%)$ in $\mathrm{CDCl}_{3}(0.15 \mathrm{~mL}, 0.2 \mathrm{M})$. While vigorously stirring at room temperature, $\mathrm{H}_{2} \mathrm{O}_{2}\left(30 \% \mathrm{w} / \mathrm{w}\right.$ in $\mathrm{H}_{2} \mathrm{O}$; $4.6 \mu \mathrm{L}, 0.05 \mathrm{mmol}, 1.50$ equiv) was added followed by DIC ( $4.6 \mu \mathrm{L}, 0.05 \mathrm{mmol}, 1.00$ equiv). After stirring for 15 $\mathrm{h}$, the reaction was quenched by addition of $\mathrm{Na}_{2} \mathrm{SO}_{3}$, then directly purified by normal-phase column chromatography (SNAP Ultra $25 \mathrm{~g}, 0-8 \% \mathrm{DCM} / \mathrm{MeOH}$ over $5 \mathrm{CV}$, then $8 \% \mathrm{DCM} / \mathrm{MeOH}$ for $2 \mathrm{CV}$, then $8-10 \%$ $\mathrm{DCM} / \mathrm{MeOH}$ for $2 \mathrm{CV}$ ) to afford racemic 61 .
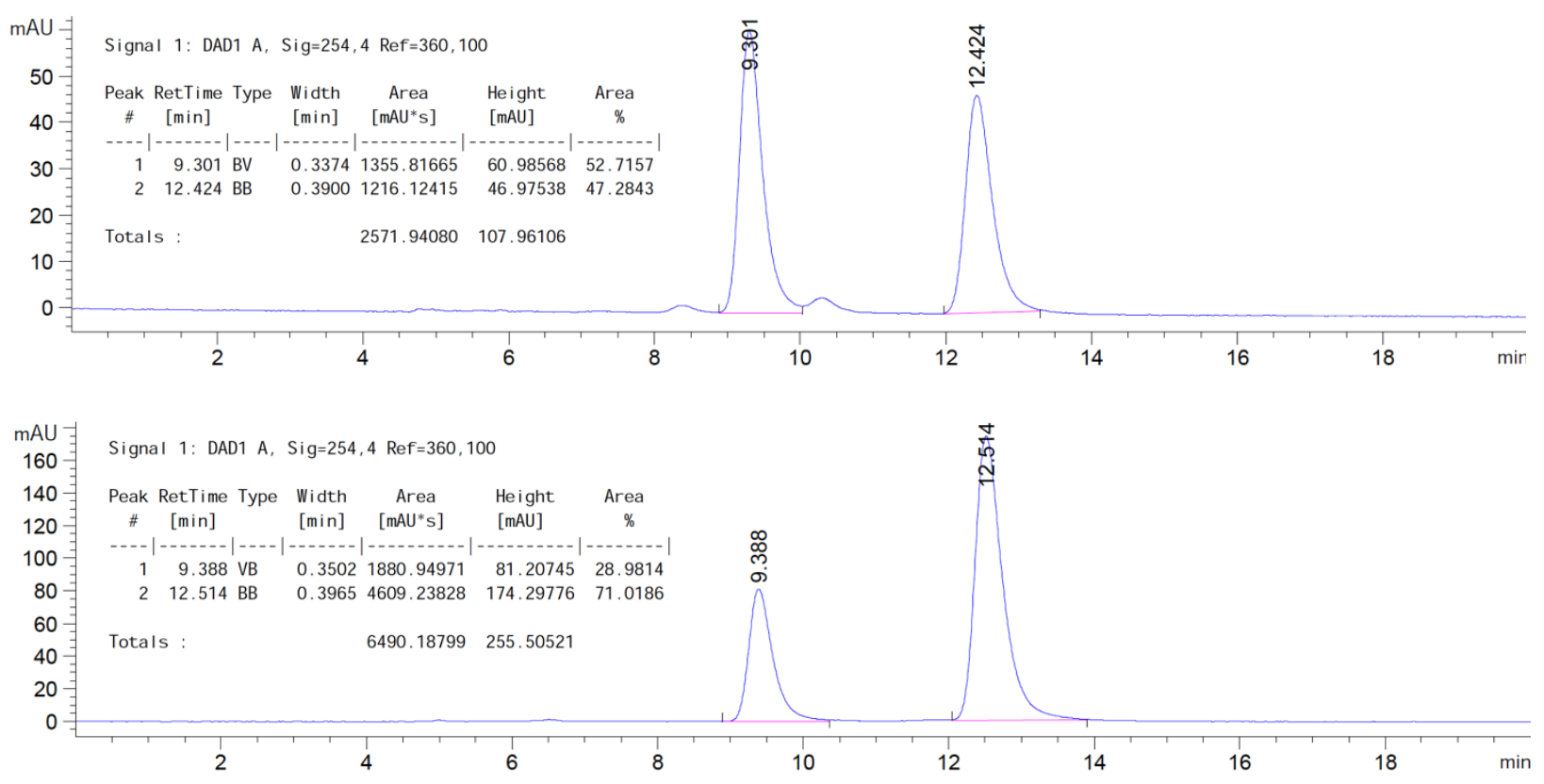


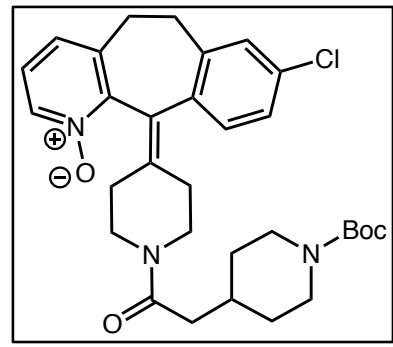

11-(1-(2-(1-( $t$-Butoxycarbonyl)piperidin-4-yl)acetyl)piperidin-4-ylidene)-8chloro-6,11-dihydro-5 $H$-benzo[5,6]cyclohepta[1,2-b]pyridine 1-oxide $(6 \mathrm{~m})$ White solid (13.3 mg, $0.024 \mathrm{mmol}, 48 \%$ yield). TLC: $\mathrm{R}_{\mathrm{f}}=0.18(10 \% \mathrm{MeOH} / \mathrm{EtOAc})$. IR (FT-ATR, neat): 1684, 1635, 1423, 1365, 1275, 1239, 1162, 1123, 993, 973, 864, $796 \mathrm{~cm}^{-1} .{ }^{1} \mathrm{H}$ NMR $\left(600 \mathrm{MHz}, \mathrm{CD}_{3} \mathrm{OD}\right) \delta 8.20(\mathrm{~d}, J=6.4 \mathrm{~Hz}, 1 \mathrm{H}), 7.50(\mathrm{dd}, J=7.7$, $3.0 \mathrm{~Hz}, 1 \mathrm{H}), 7.40-7.36(\mathrm{~m}, 1 \mathrm{H}), 7.32(\mathrm{t}, J=8.0 \mathrm{~Hz}, 1 \mathrm{H}), 7.21-7.18(\mathrm{~m}, 2 \mathrm{H}), 4.09$ - $4.02(\mathrm{~m}, 2 \mathrm{H}), 3.93(\mathrm{tt}, J=11.4,5.3 \mathrm{~Hz}, 1 \mathrm{H}), 3.80(\mathrm{dq}, J=11.0,5.7 \mathrm{~Hz}, 1 \mathrm{H}), 3.52$ $3.36(\mathrm{~m}, 4 \mathrm{H}), 2.94(\mathrm{dtd}, J=21.7,11.7,10.7,4.1 \mathrm{~Hz}, 2 \mathrm{H}), 2.76(\mathrm{bs}, 2 \mathrm{H}), 2.55$ (dddd, $J=42.6,13.6,8.6,4.4 \mathrm{~Hz}, 1 \mathrm{H}), 2.46-2.23(\mathrm{~m}, 4 \mathrm{H}), 2.08-1.90(\mathrm{~m}, 2 \mathrm{H}), 1.73(\mathrm{t}, J=11.2 \mathrm{~Hz}, 2 \mathrm{H}), 1.45(\mathrm{~s}, 9 \mathrm{H})$, $1.19-1.07(\mathrm{~m}, 2 \mathrm{H}) .{ }^{13} \mathrm{C}$ NMR $\left(151 \mathrm{MHz}, \mathrm{CD}_{3} \mathrm{OD}\right) \delta 172.6,156.5,150.7 / 150.6(1 \mathrm{C}), 142.5 / 142.4(1 \mathrm{C})$, 140.5/140.4 (1C), 140.4/140.3 (1C), 138.5/138.5 (1C), 134.9/134.9 (1C), 134.8, 133.5, 131.3/131.3 (1C), 130.2/130.2 (1C), 127.0, 126.2, 124.7, 80.9, 49.0, 47.3, 46.9, 43.5, 43.1, 40.4, 34.6/34.6 (1C), 33.4, 33.1, 32.8, 32.6, 31.9, 31.1, 28.7 [Note: two conformations were detected via ${ }^{13} \mathrm{C}$ NMR]. HRMS (ESI) $\mathrm{m} / \mathrm{z}:[\mathrm{M}+\mathrm{H}]^{+}$calcd for $\mathrm{C}_{31} \mathrm{H}_{39} \mathrm{ClN}_{3} \mathrm{O}_{4}{ }^{+}$552.2624, found 552.2635. HPLC (Chiralpak IB column, $60 \% \mathrm{EtOH} /$ hexanes, $0.7 \mathrm{~mL} / \mathrm{min}$ flowrate, $2 \mu \mathrm{L}$ injection, $25^{\circ} \mathrm{C}, 254 \mathrm{~nm}$ ): minor enantiomer $t_{\mathrm{R}}=8.3 \mathrm{~min}$, major enantiomer $t_{\mathrm{R}}=10.1 \mathrm{~min}$. $[\boldsymbol{\alpha}]_{589}^{\mathbf{2 0}}$ $\left(\mathrm{c}=0.58, \mathrm{CHCl}_{3} ; 74: 26 \mathrm{er}\right):+56.2$.
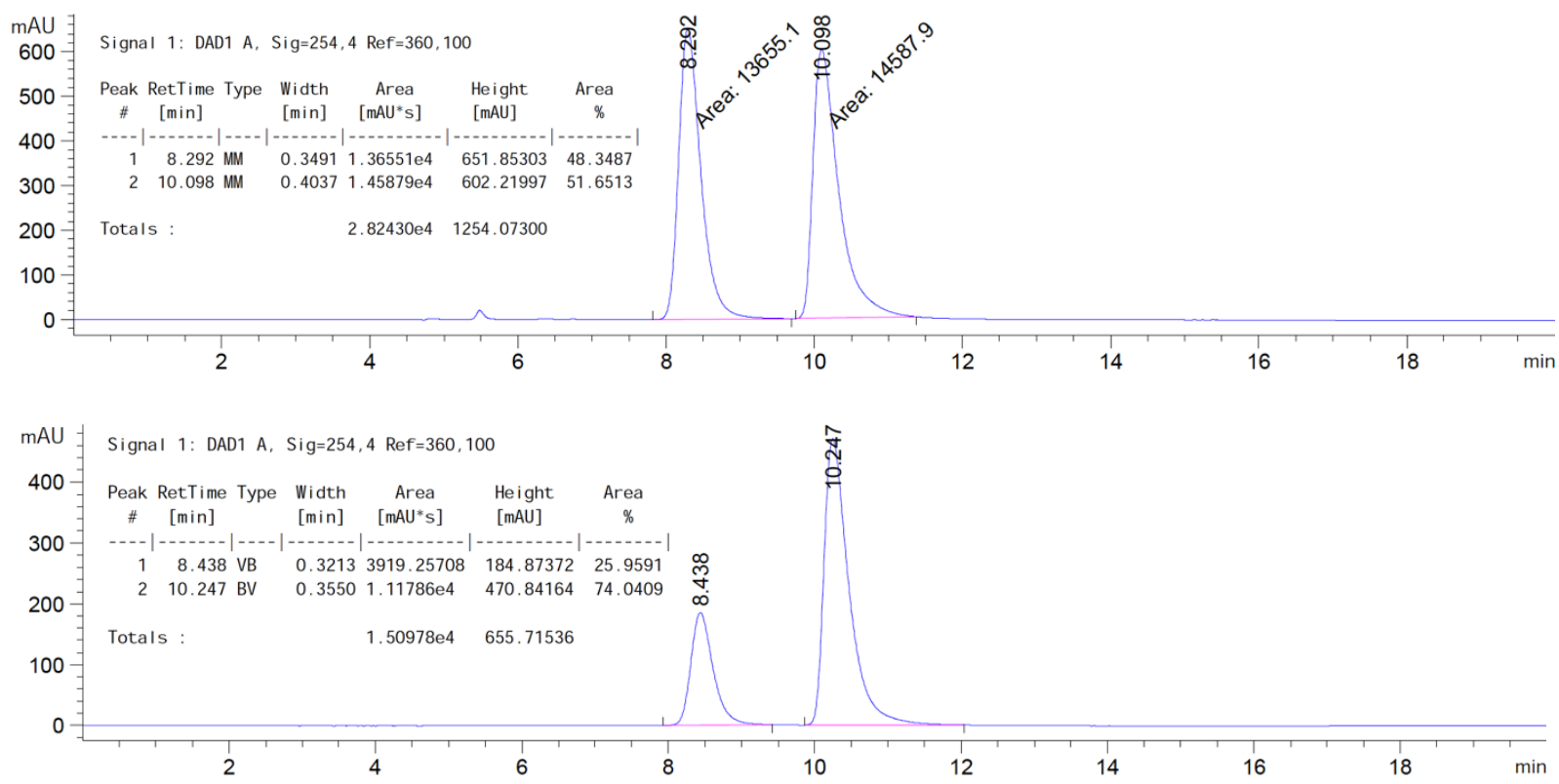

\section{Hammett Plot}

The general procedure (S11) for catalysis was employed using P2 (1.7 mg, $10 \mathrm{~mol} \%)$ with substrates $\mathbf{1 b}-\mathbf{g}(0.03$ $\mathrm{mmol})$. An average of two trials is presented in Table S1.

Table S1. Hammett plot data.

\begin{tabular}{cccc} 
Substrate & er, $\mathbf{6}$ & $\Delta \Delta \mathrm{G}^{\ddagger}(\mathrm{kcal} / \mathrm{mol})$ & Hammett Value $\left(\sigma_{\text {para }}\right)^{2}$ \\
\hline $\mathbf{1 b}(\mathrm{H})$ & $88.9: 11.1$ & 1.22 & 0.00 \\
$\mathbf{1 c}(\mathrm{OMe})$ & $86.5: 13.5$ & 1.09 & -0.27 \\
1d $(\mathrm{Cl})$ & $91.4: 8.6$ & 1.39 & 0.23 \\
$\mathbf{1 e}\left(\mathrm{CF}_{3}\right)$ & $93.5: 6.5$ & 1.56 & 0.54 \\
$\mathbf{1 f}(\mathrm{CN})$ & $94.2: 5.8$ & 1.63 & 0.66 \\
$\mathbf{1 g}\left(\mathrm{NO}_{2}\right)$ & $94.7: 5.3$ & 1.69 & 0.78
\end{tabular}




\section{VI. ${ }^{1} \mathrm{H},{ }^{13} \mathrm{C}$, and ${ }^{19} \mathrm{~F}$ NMR Spectra}

${ }^{1} \mathrm{H} \operatorname{NMR}\left(600 \mathrm{MHz}, \mathrm{CDCl}_{3}\right)-\mathbf{P} 3$
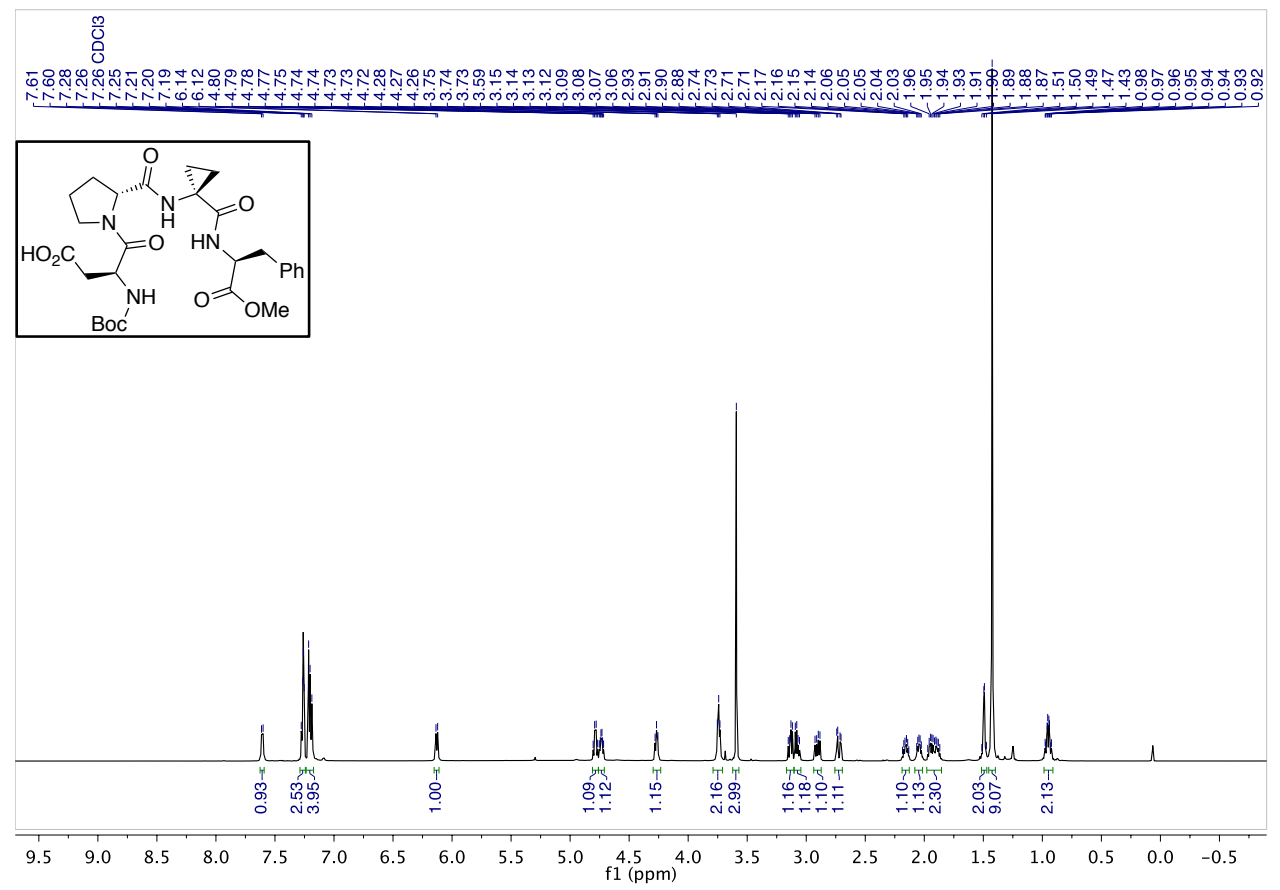

${ }^{13} \mathrm{C} \mathrm{NMR}\left(151 \mathrm{MHz}, \mathrm{CDCl}_{3}\right)-\mathbf{P} 3$

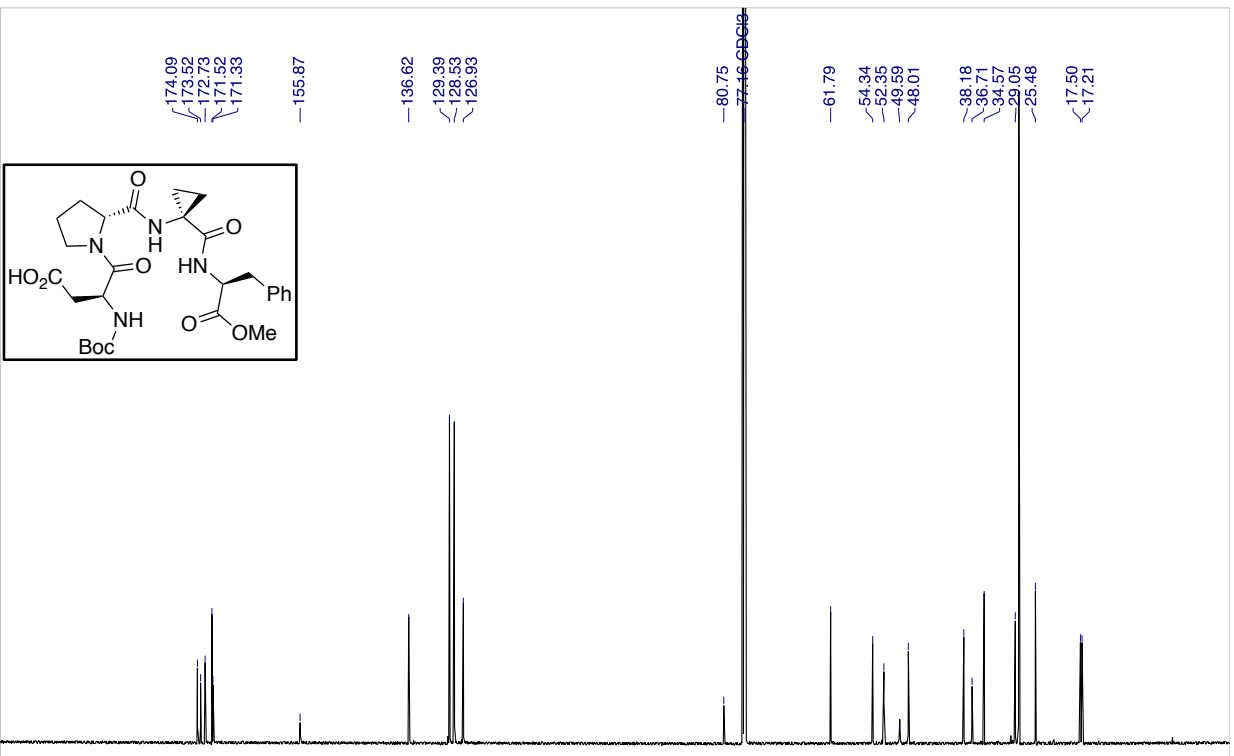

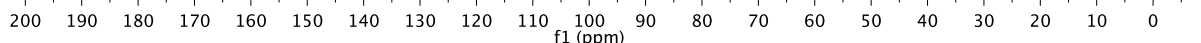


${ }^{1} \mathrm{H}$ NMR $\left(600 \mathrm{MHz}, \mathrm{CDCl}_{3}\right)$ - P4

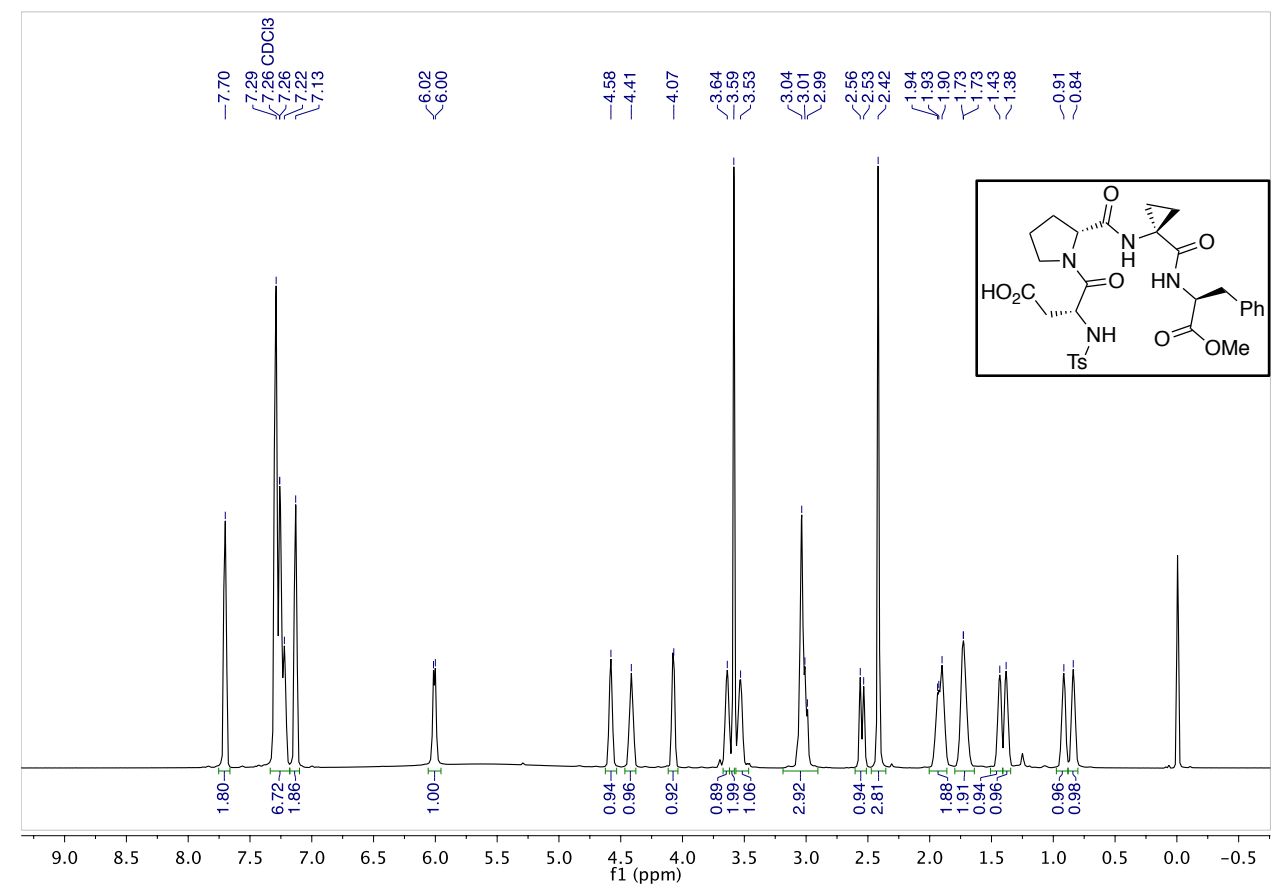

${ }^{13} \mathrm{C}$ NMR (151 MHz, $\left.\mathrm{CDCl}_{3}\right)$ - P4

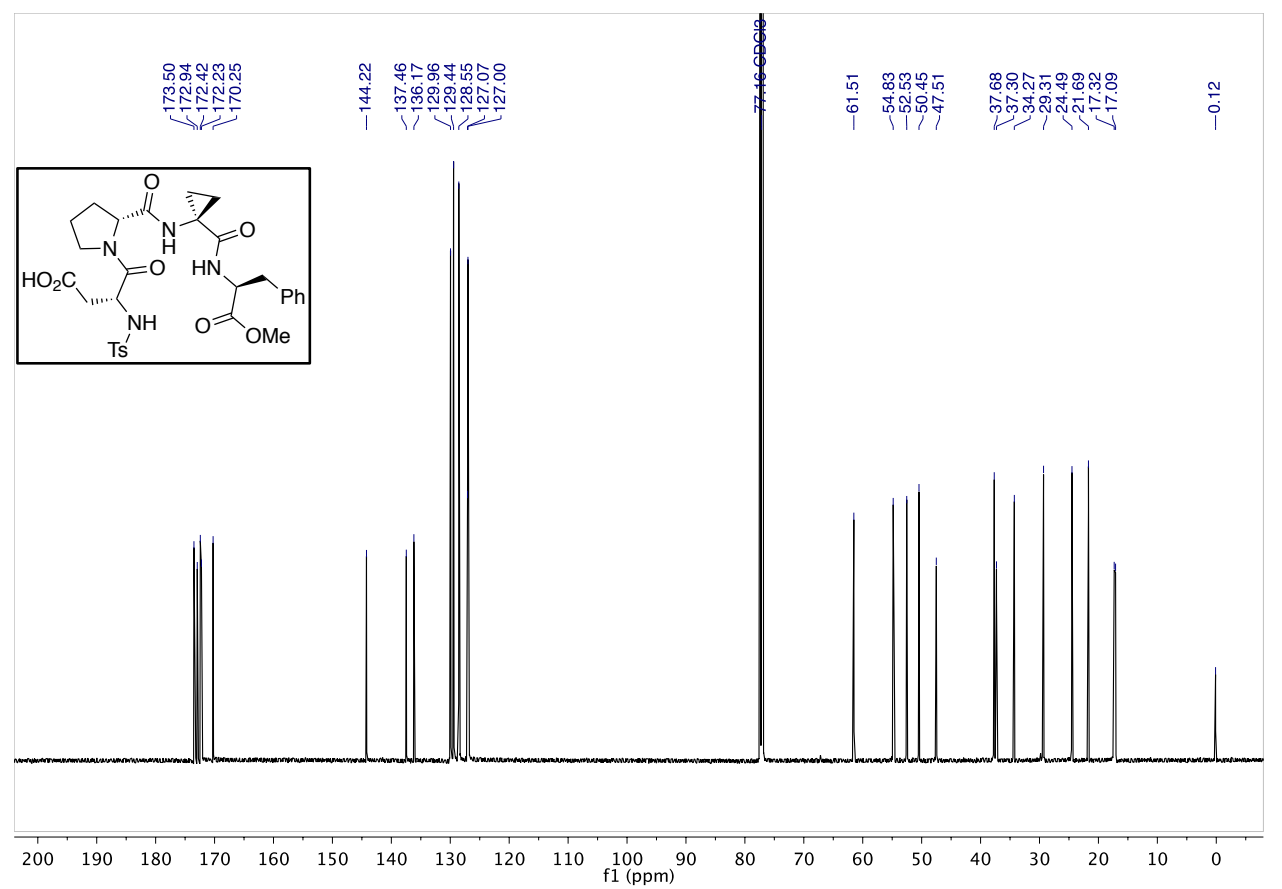


${ }^{1} \mathrm{H} \mathrm{NMR}\left(600 \mathrm{MHz}, \mathrm{CDCl}_{3}\right)-\mathbf{P 5}$

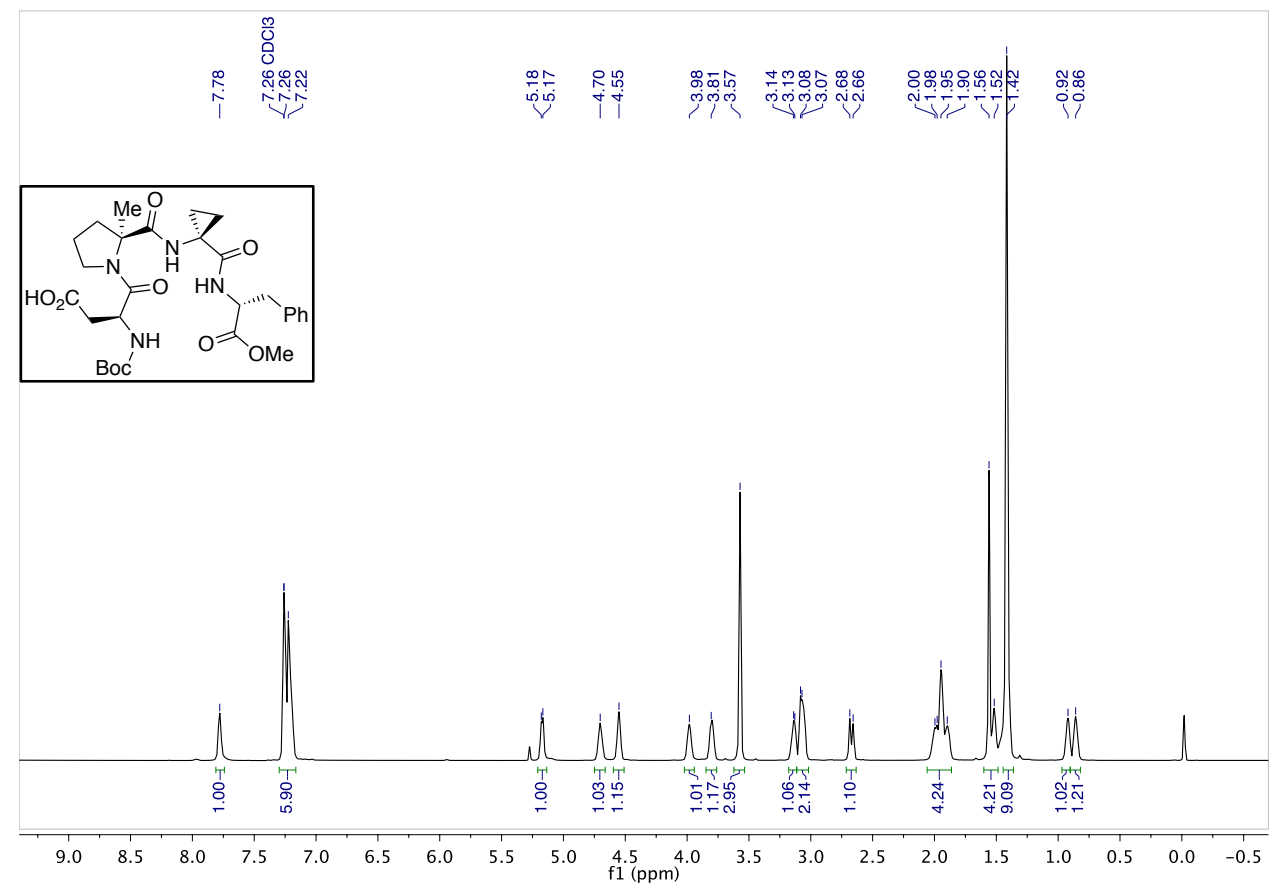

${ }^{13} \mathrm{C} \operatorname{NMR}\left(151 \mathrm{MHz}, \mathrm{CDCl}_{3}\right)-\mathbf{P 5}$

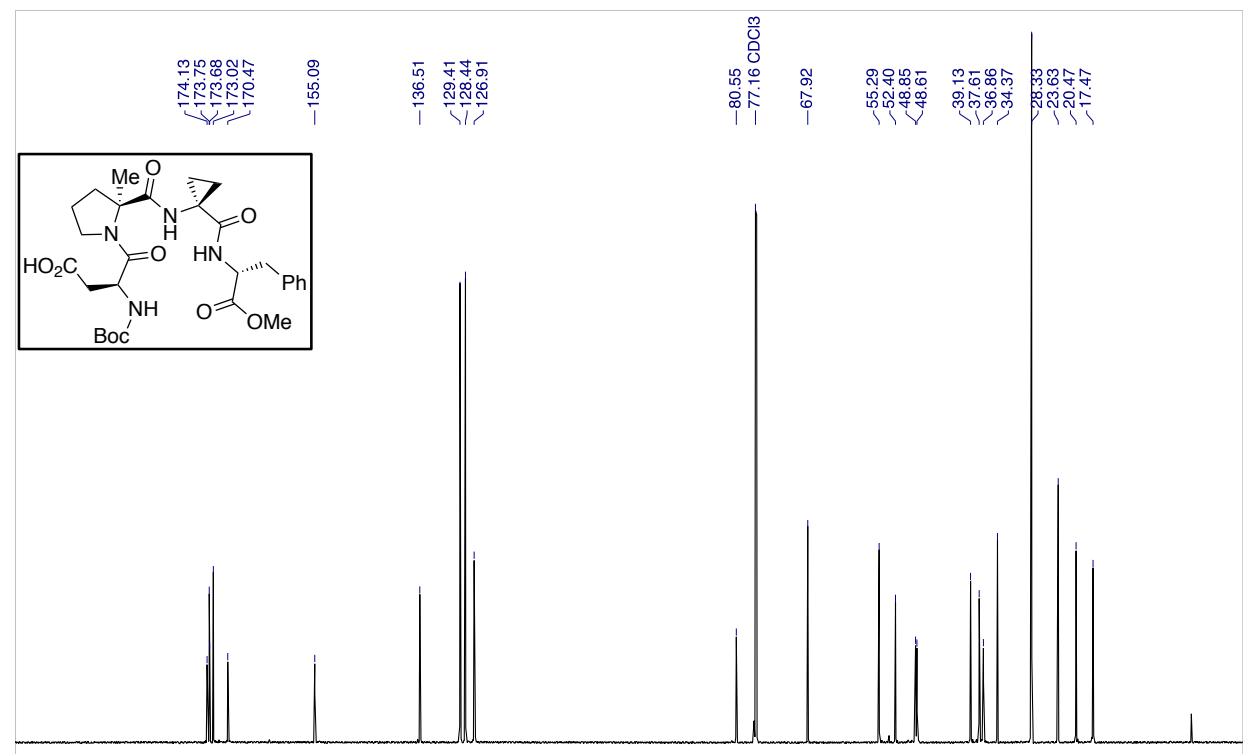

$\begin{array}{lllllllllllllllllllllllllll}200 & 190 & 180 & 170 & 160 & 150 & 140 & 130 & 120 & 110 & 100 & 90 & 80 & 70 & 60 & 50 & 40 & 30 & 20 & 10 & 0\end{array}$ 
${ }^{1} \mathrm{H} \mathrm{NMR}\left(600 \mathrm{MHz}, \mathrm{CDCl}_{3}\right)-\mathbf{P} 6$

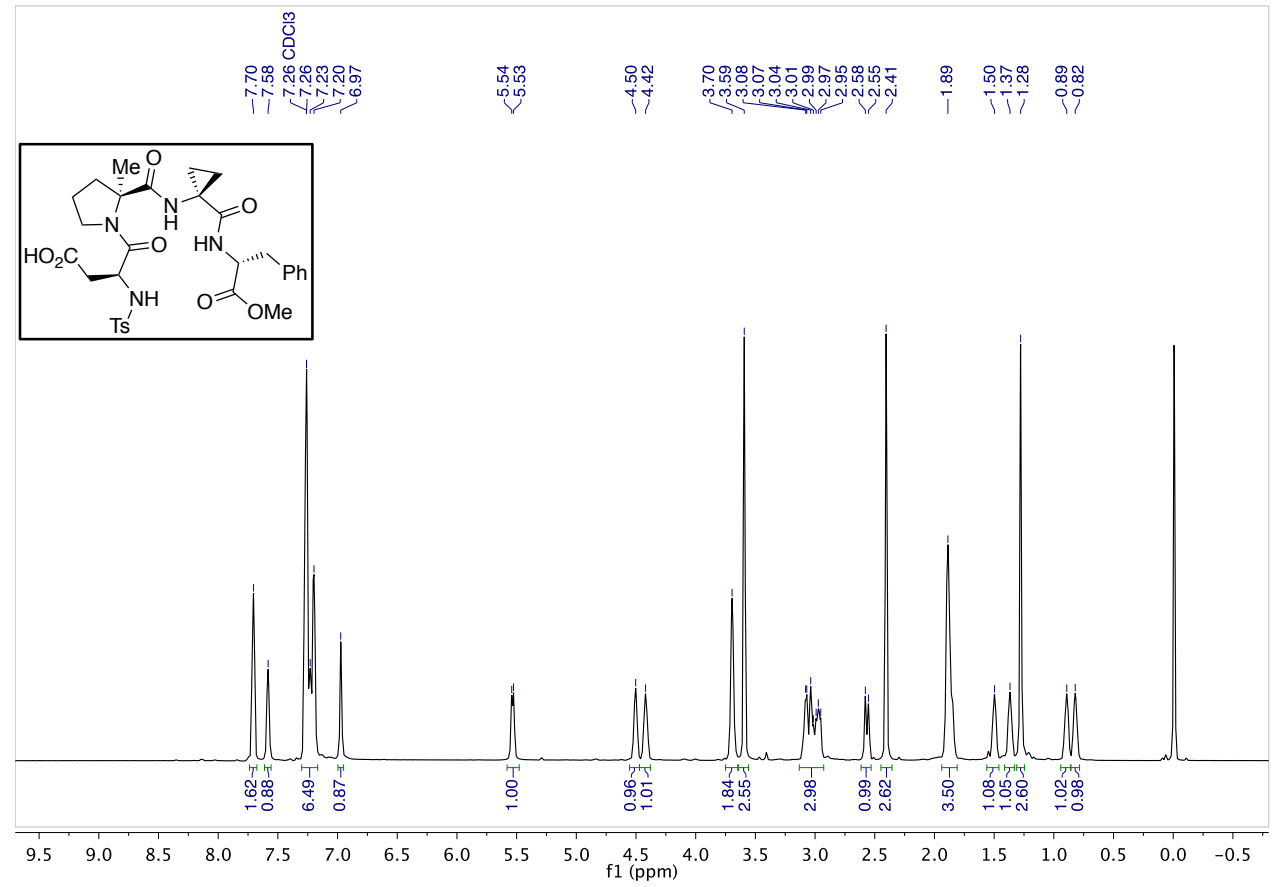

${ }^{13} \mathrm{C} \mathrm{NMR}\left(151 \mathrm{MHz}, \mathrm{CDCl}_{3}\right)-\mathbf{P 6}$

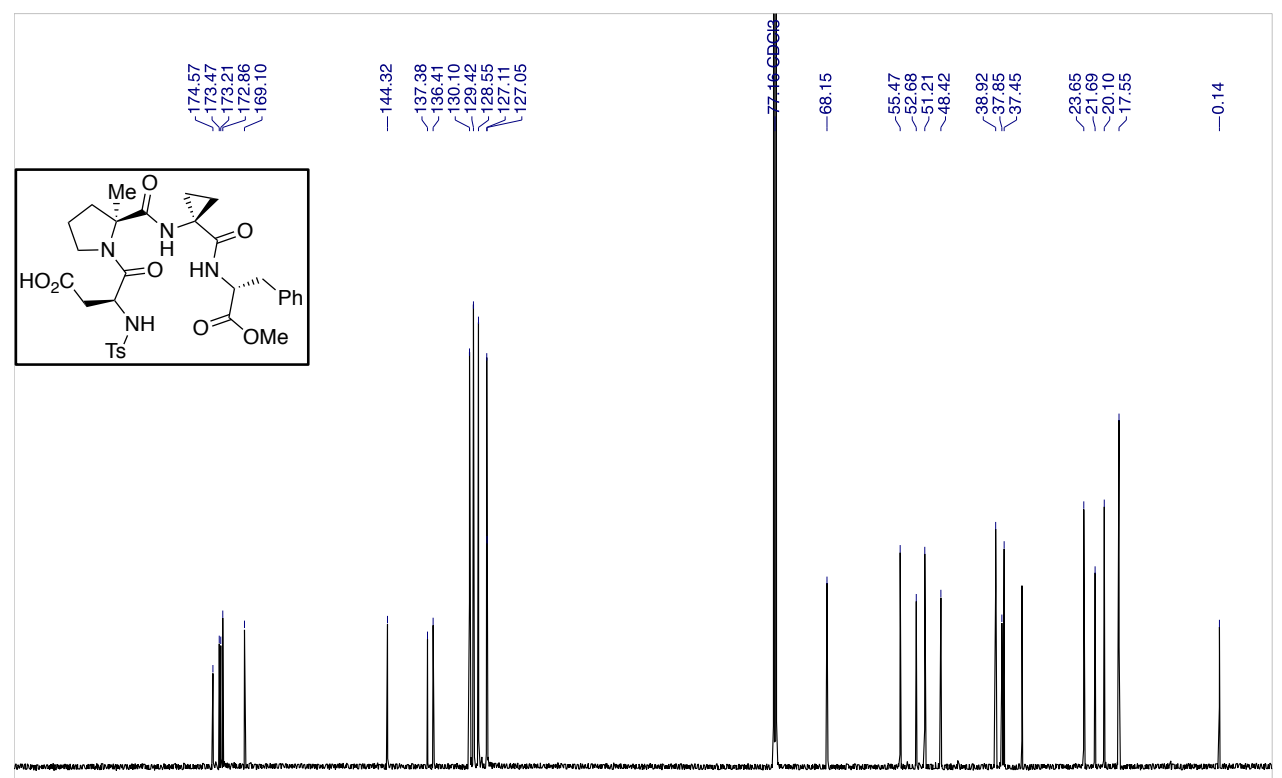

$\begin{array}{llllllllllllllllllllll}200 & 190 & 180 & 170 & 160 & 150 & 140 & 130 & 120 & 110 & 100 & 90 & 80 & 70 & 60 & 50 & 40 & 30 & 20 & 10 & 0\end{array}$ 
${ }^{1} \mathrm{H} \mathrm{NMR}\left(600 \mathrm{MHz}, \mathrm{CD}_{3} \mathrm{OD}\right)-\mathbf{1 b}$

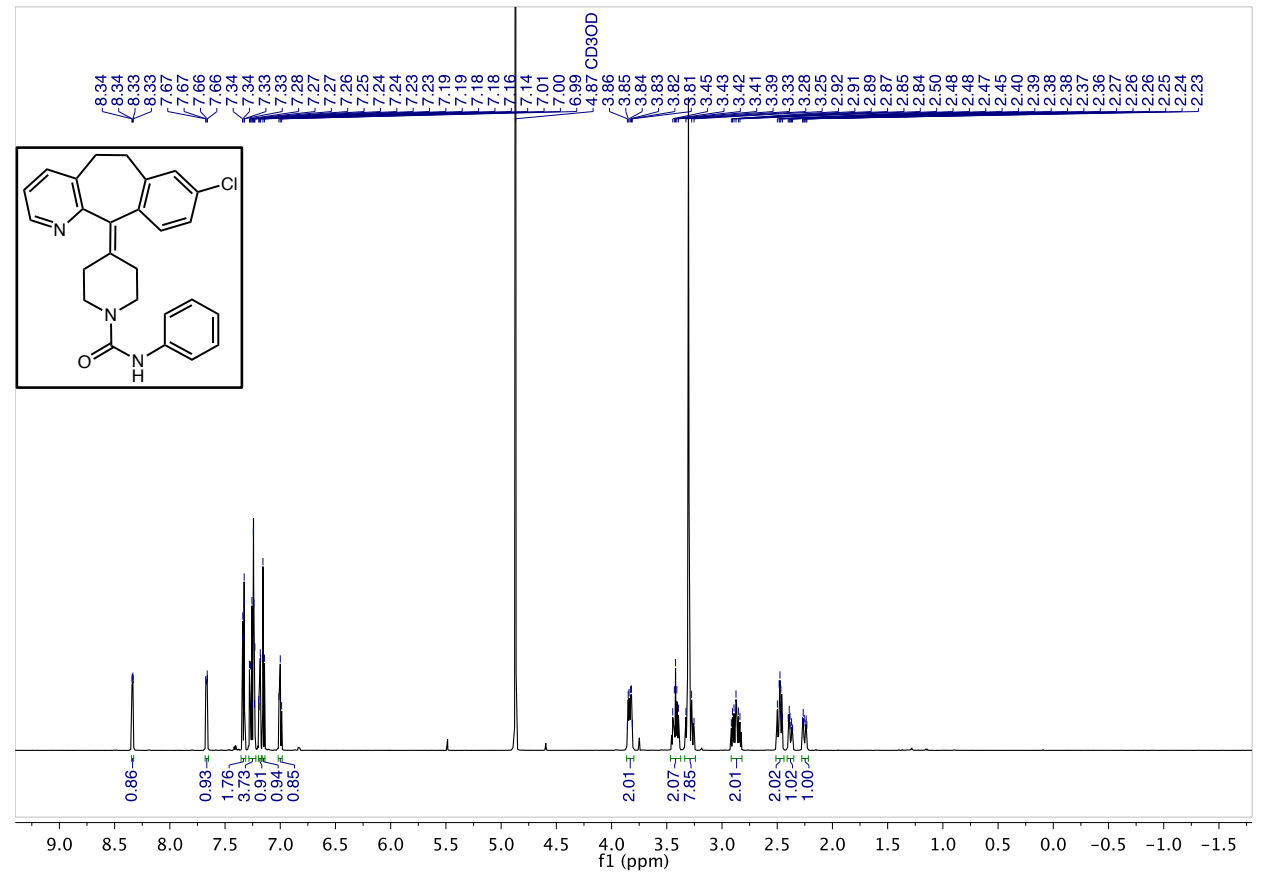

${ }^{13} \mathrm{C}$ NMR $\left(151 \mathrm{MHz}, \mathrm{CD}_{3} \mathrm{OD}\right)-\mathbf{1 b}$

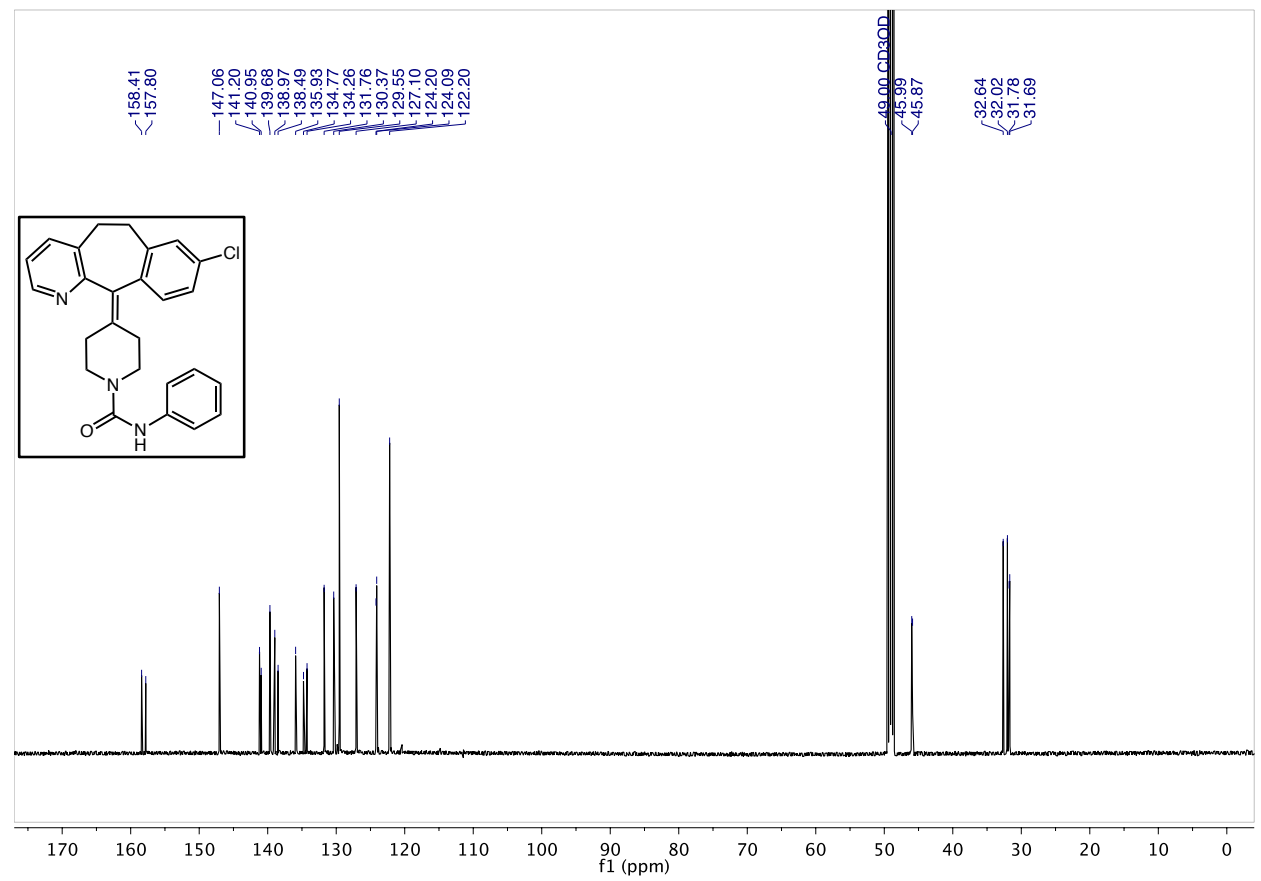


${ }^{1} \mathrm{H} \mathrm{NMR}\left(600 \mathrm{MHz}, \mathrm{CD}_{3} \mathrm{OD}\right)-\mathbf{6 b}$

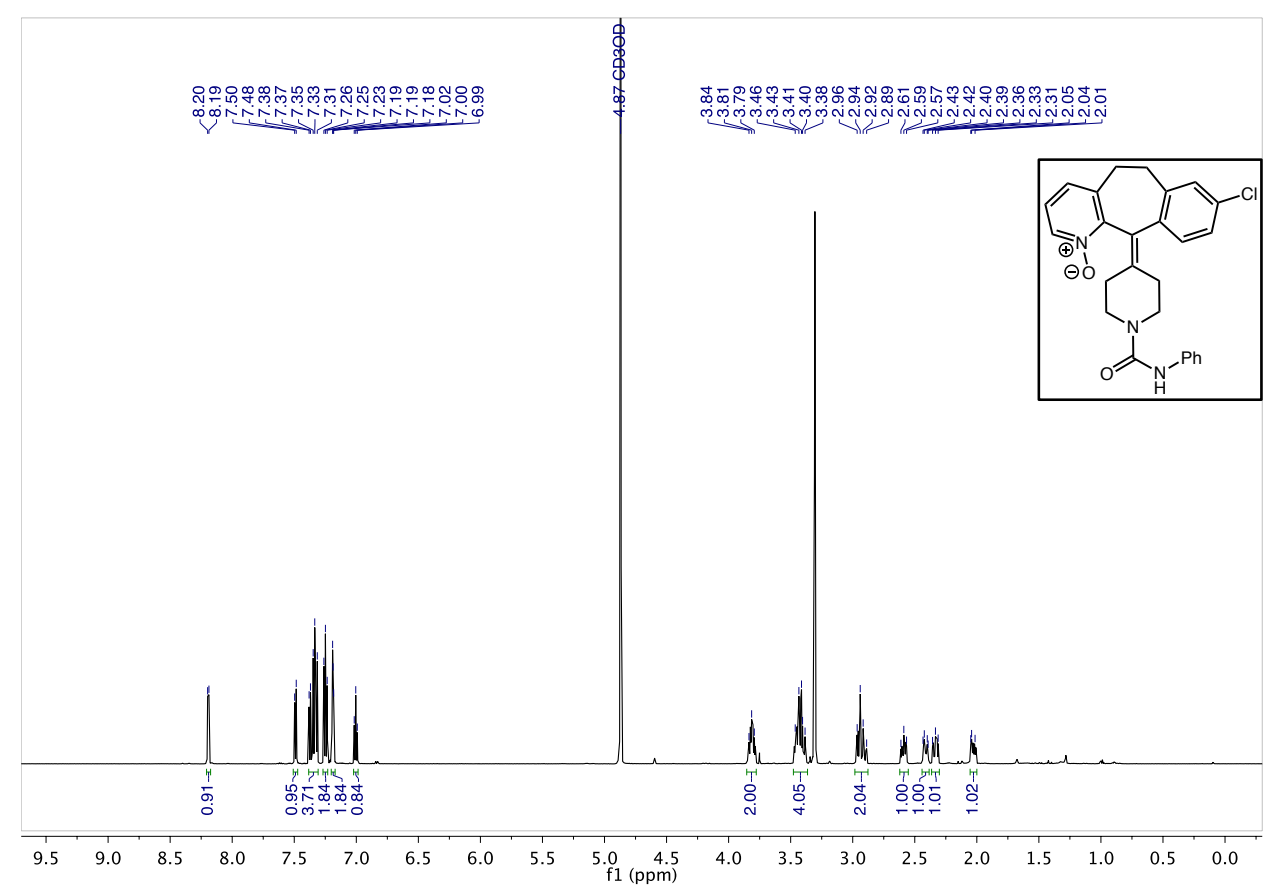

${ }^{13} \mathrm{C}$ NMR $\left(151 \mathrm{MHz}, \mathrm{CD}_{3} \mathrm{OD}\right)-6 \mathbf{b}$

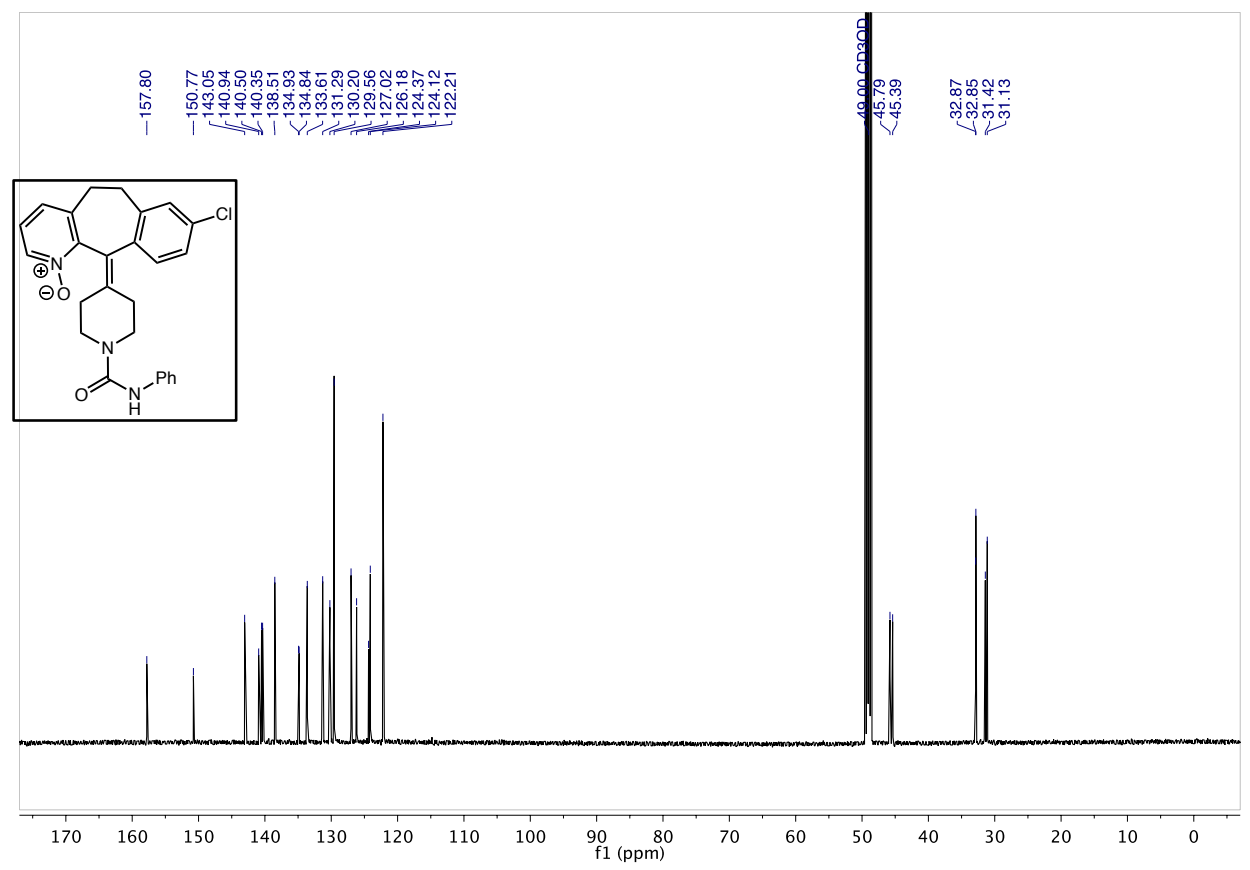


${ }^{1} \mathrm{H} \mathrm{NMR}\left(600 \mathrm{MHz}, \mathrm{CD}_{3} \mathrm{OD}\right)-1 \mathrm{c}$

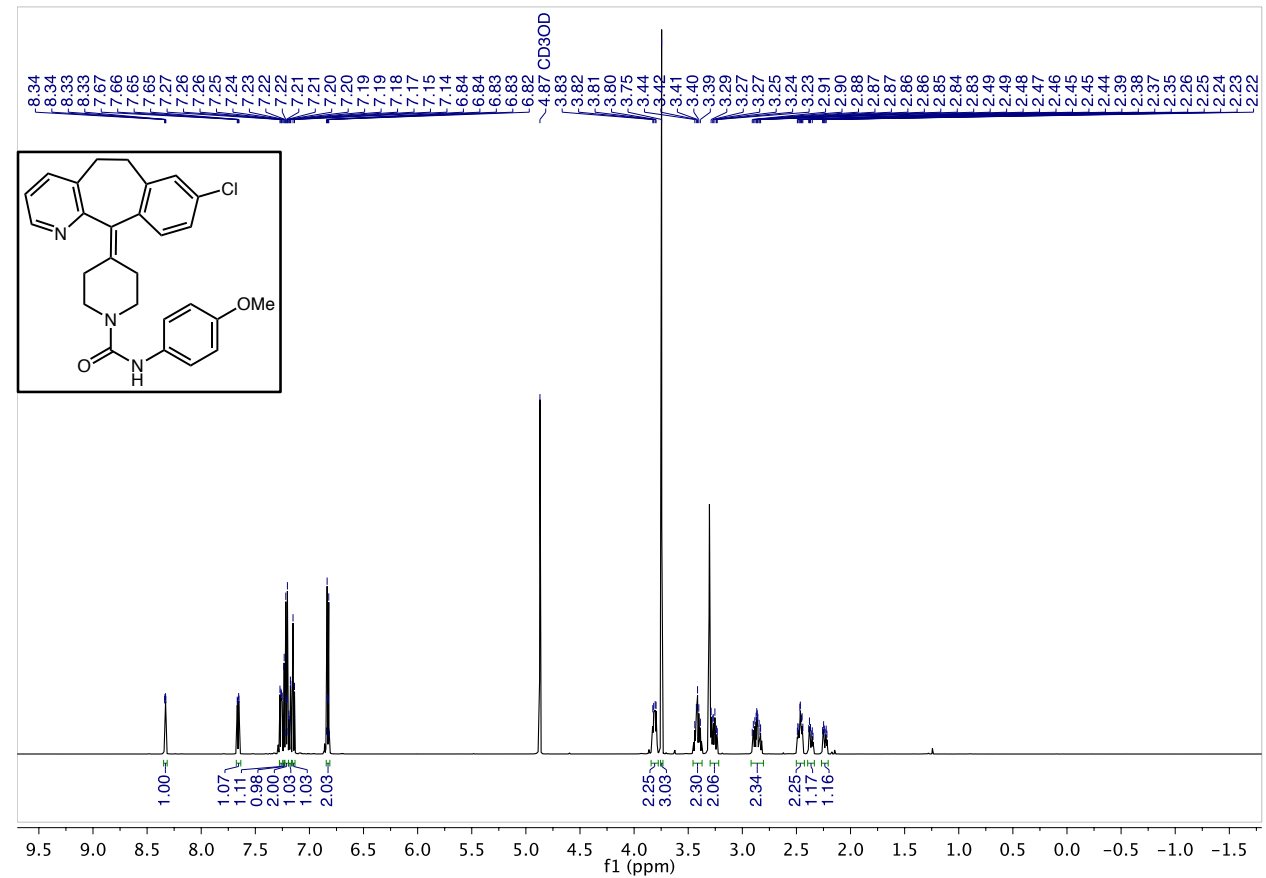

${ }^{13} \mathrm{C}$ NMR $\left(151 \mathrm{MHz}, \mathrm{CD}_{3} \mathrm{OD}\right)-\mathbf{1 c}$

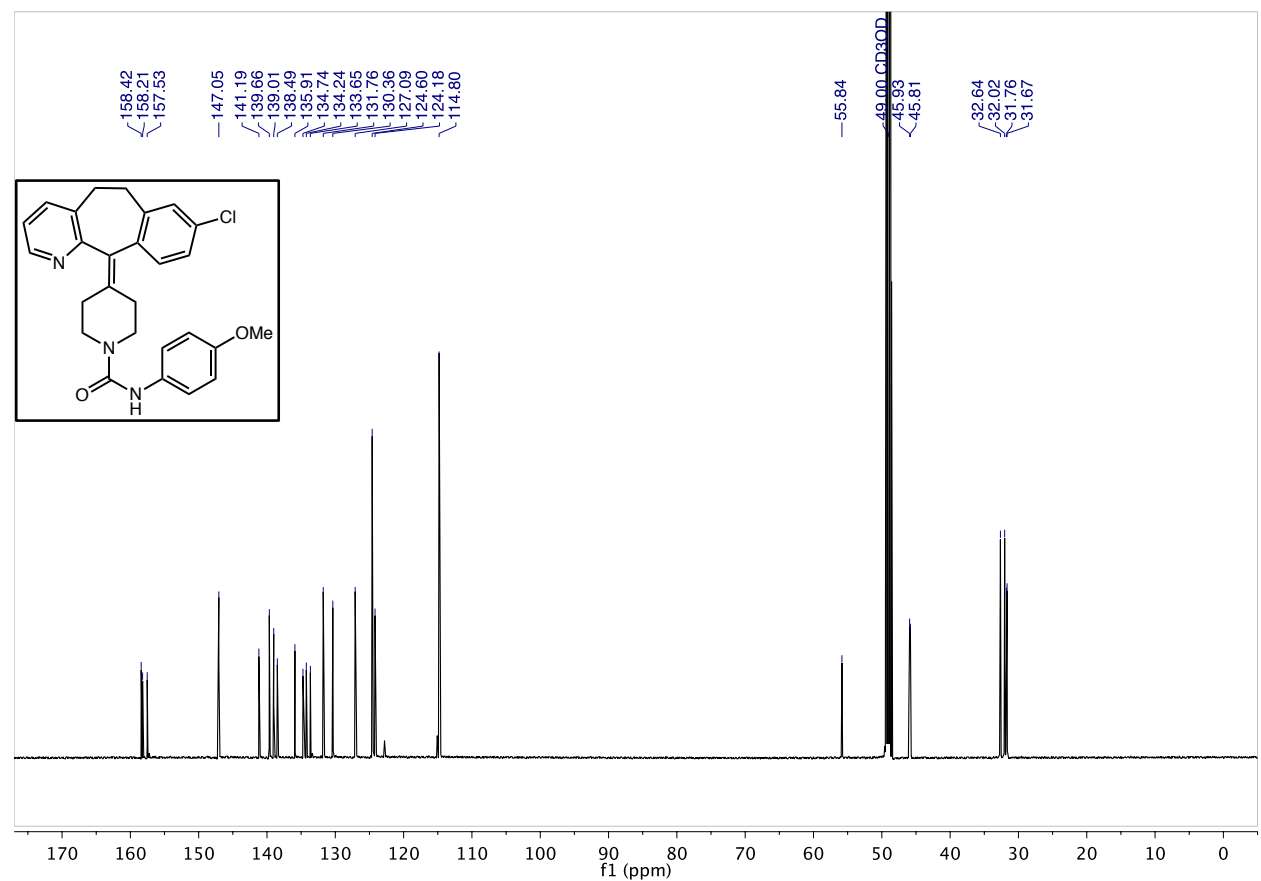


${ }^{1} \mathrm{H} \mathrm{NMR}\left(600 \mathrm{MHz}, \mathrm{CD}_{3} \mathrm{OD}\right)-6 \mathrm{c}$

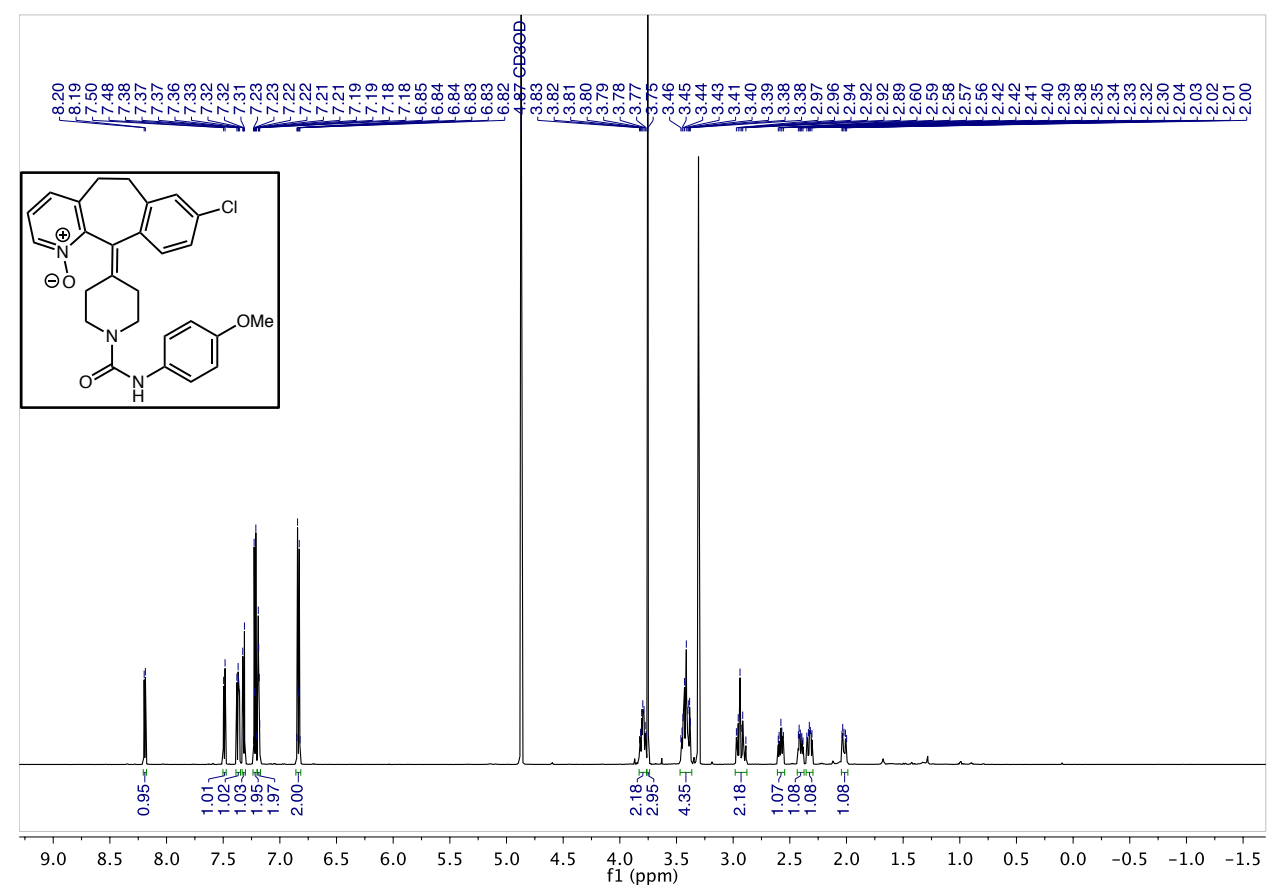

${ }^{13} \mathrm{C}$ NMR $\left(151 \mathrm{MHz}, \mathrm{CD}_{3} \mathrm{OD}\right)-\mathbf{6 c}$

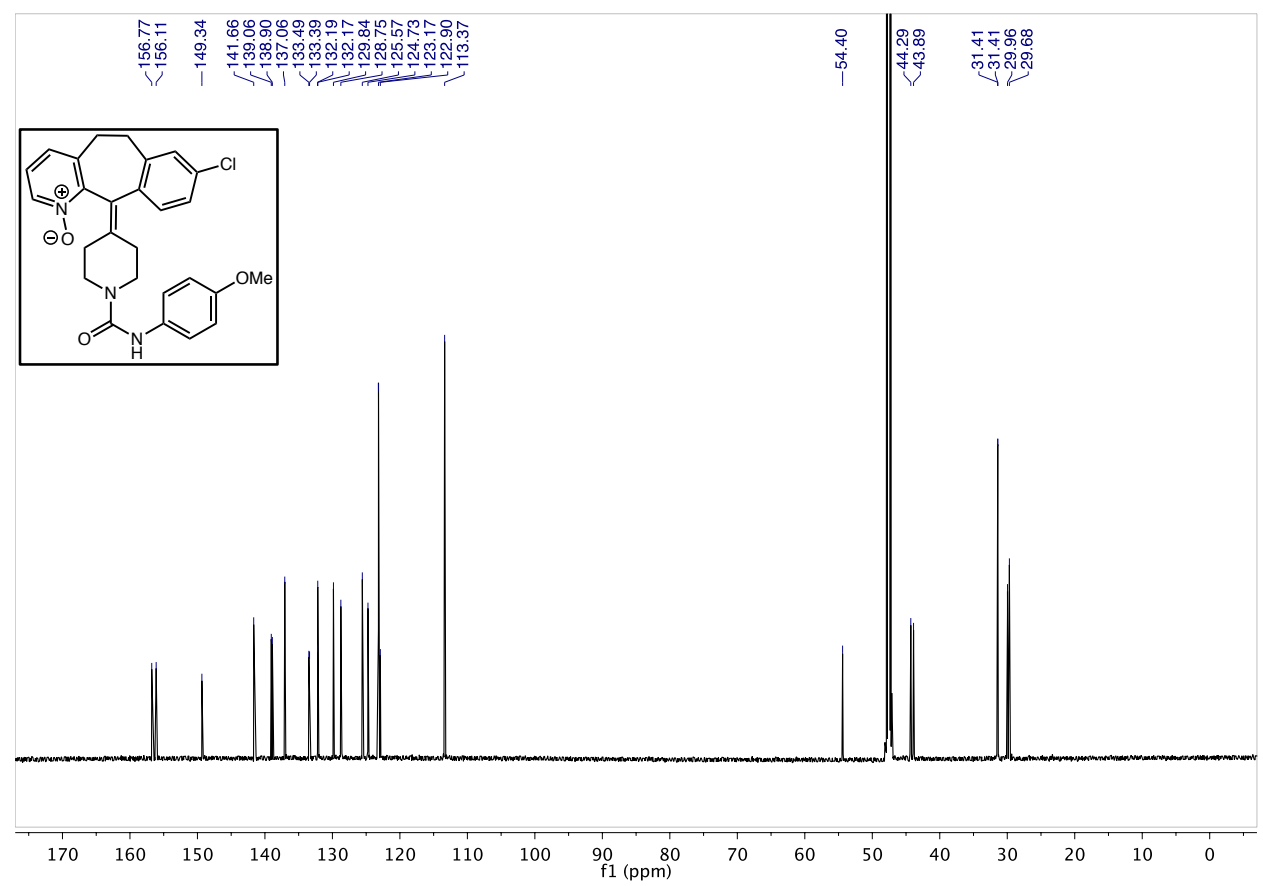


${ }^{1} \mathrm{H}$ NMR $\left(600 \mathrm{MHz}, \mathrm{CD}_{3} \mathrm{OD}\right)-\mathbf{1 d}$

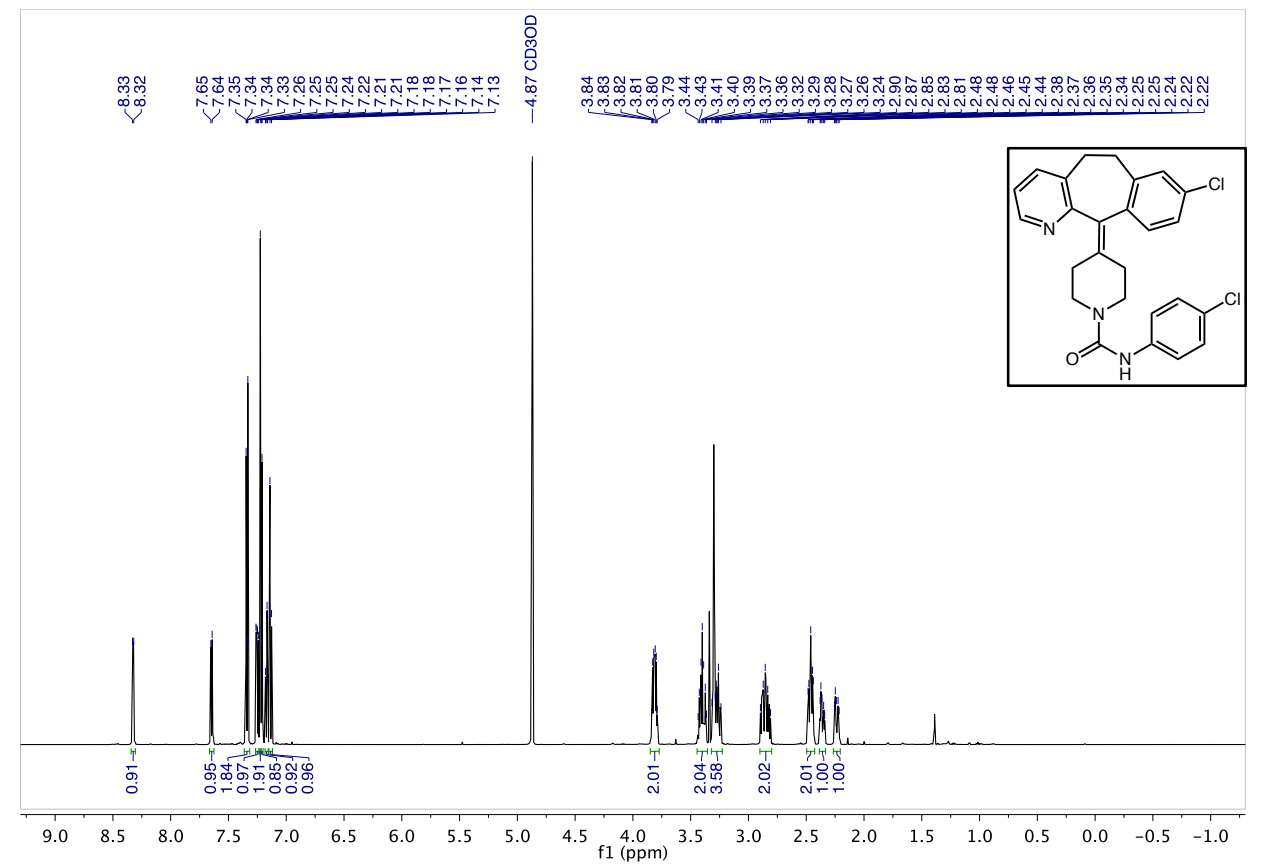

${ }^{13} \mathrm{C}$ NMR $\left(151 \mathrm{MHz}, \mathrm{CD}_{3} \mathrm{OD}\right)-\mathbf{1 d}$

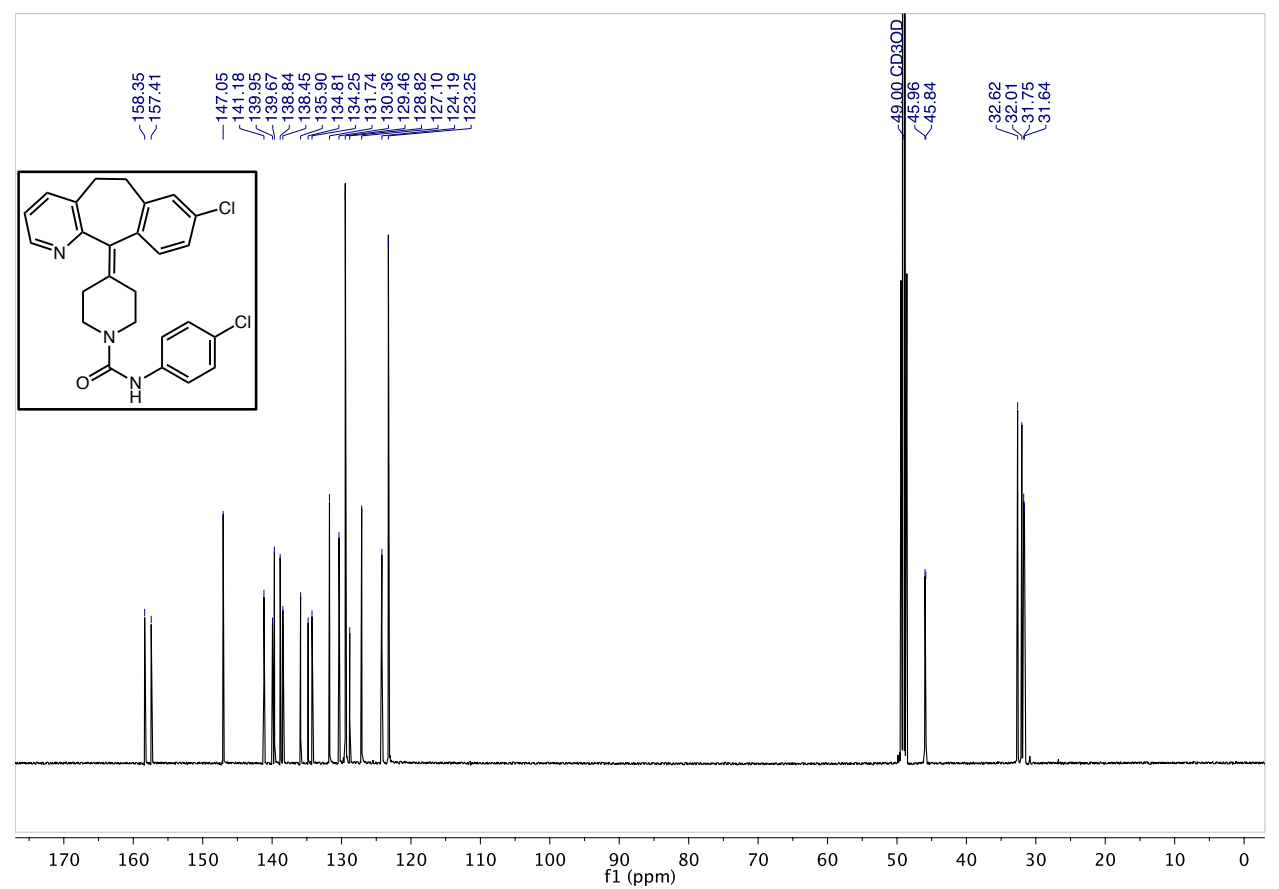


${ }^{1} \mathrm{H}$ NMR (600 MHz, $\left.\mathrm{CD}_{3} \mathrm{OD}\right)-\mathbf{6 d}$

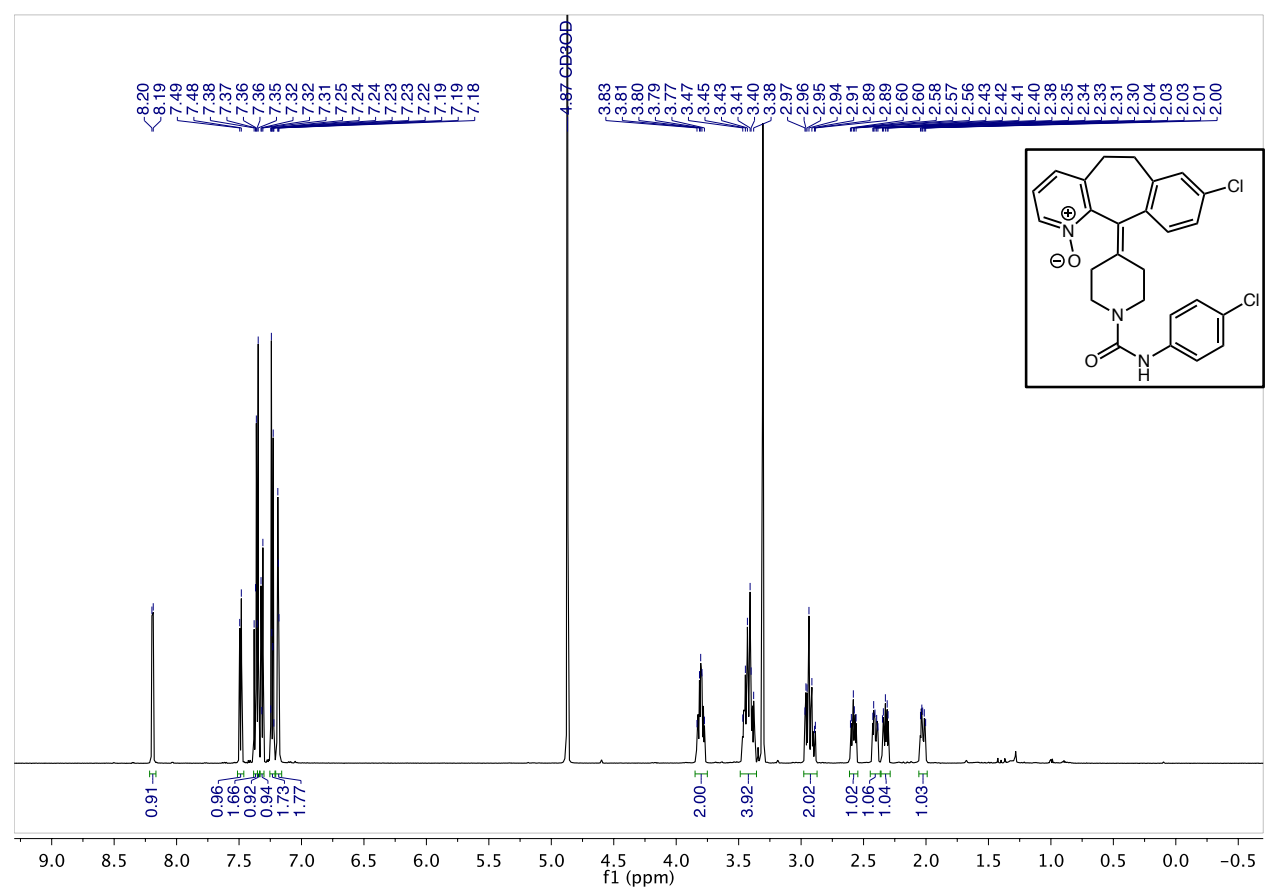

${ }^{13} \mathrm{C}$ NMR $\left(151 \mathrm{MHz}, \mathrm{CD}_{3} \mathrm{OD}\right)-6 \mathrm{~d}$

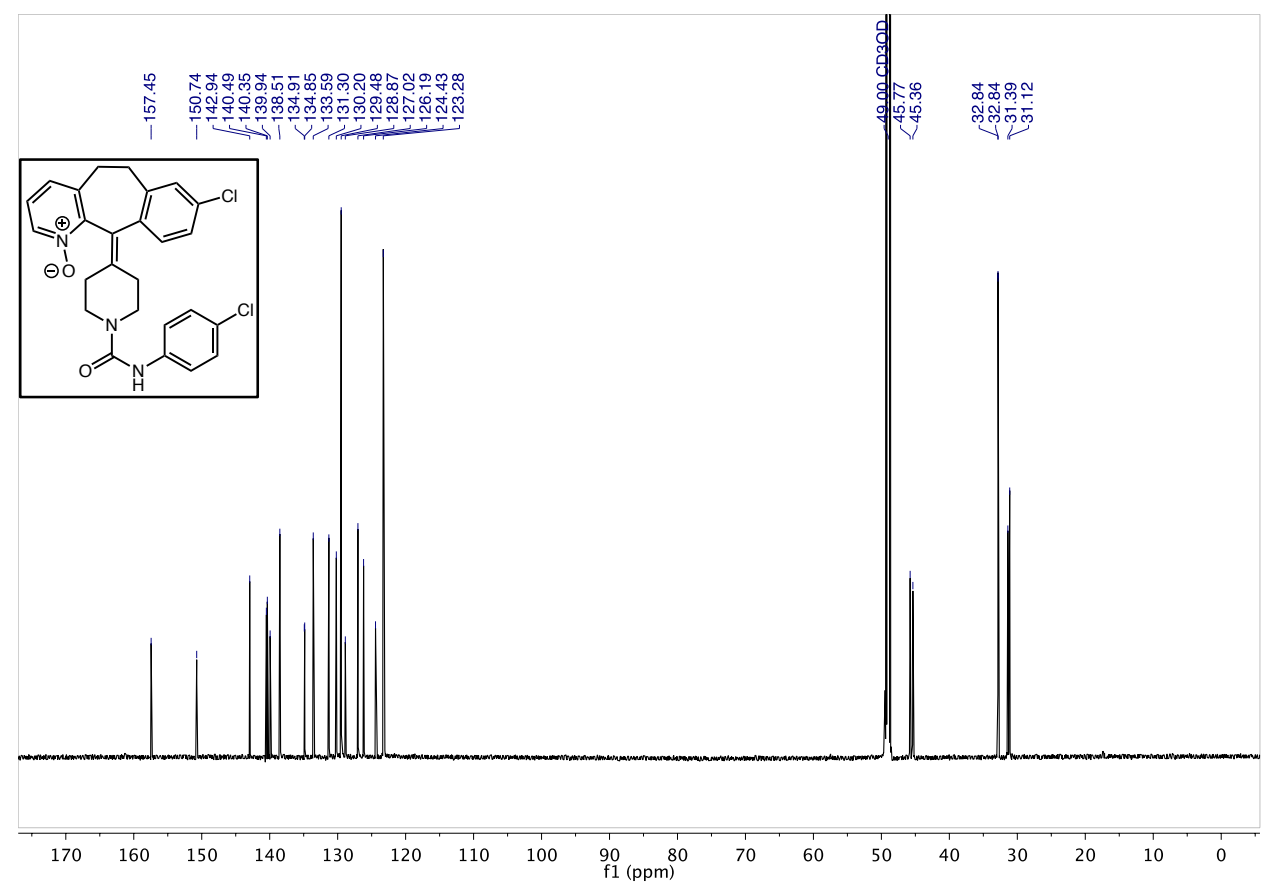


${ }^{1} \mathrm{H} \mathrm{NMR}\left(500 \mathrm{MHz}, \mathrm{CD}_{3} \mathrm{OD}\right)$ - $\mathbf{1 f}$

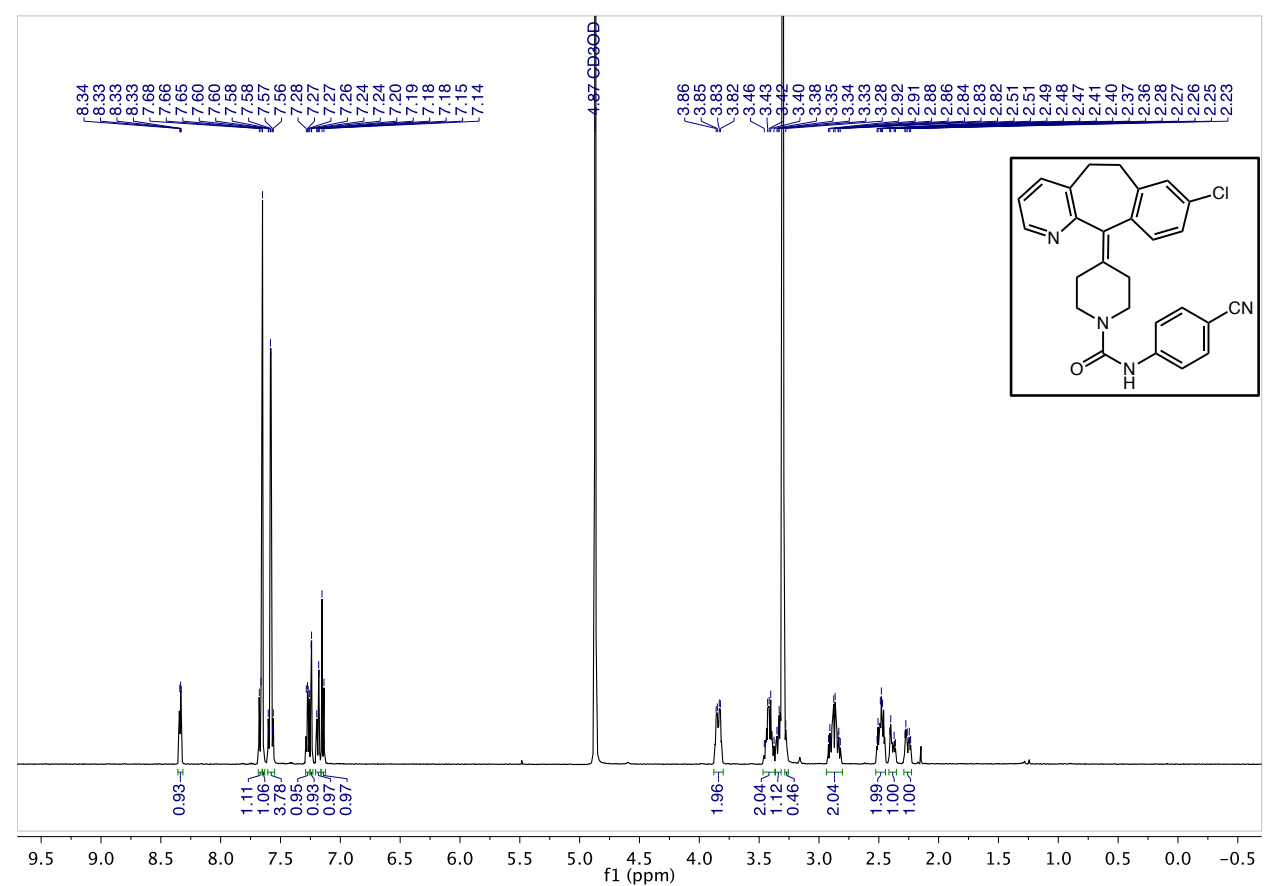

${ }^{13} \mathrm{C}$ NMR $\left(151 \mathrm{MHz}, \mathrm{CD}_{3} \mathrm{OD}\right)-\mathbf{1 f}$

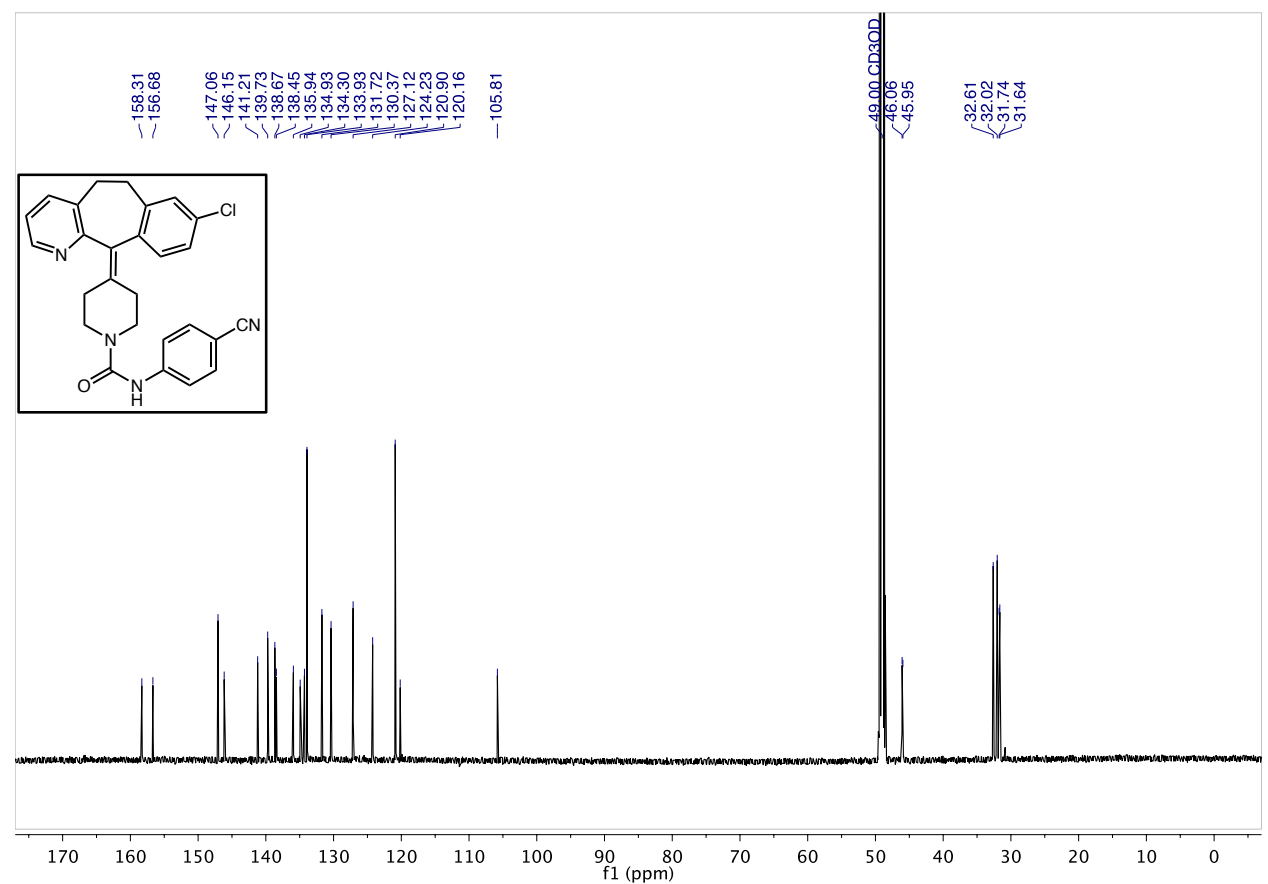


${ }^{1} \mathrm{H}$ NMR $\left(600 \mathrm{MHz}, \mathrm{CD}_{3} \mathrm{OD}\right)-\mathbf{6 f}$

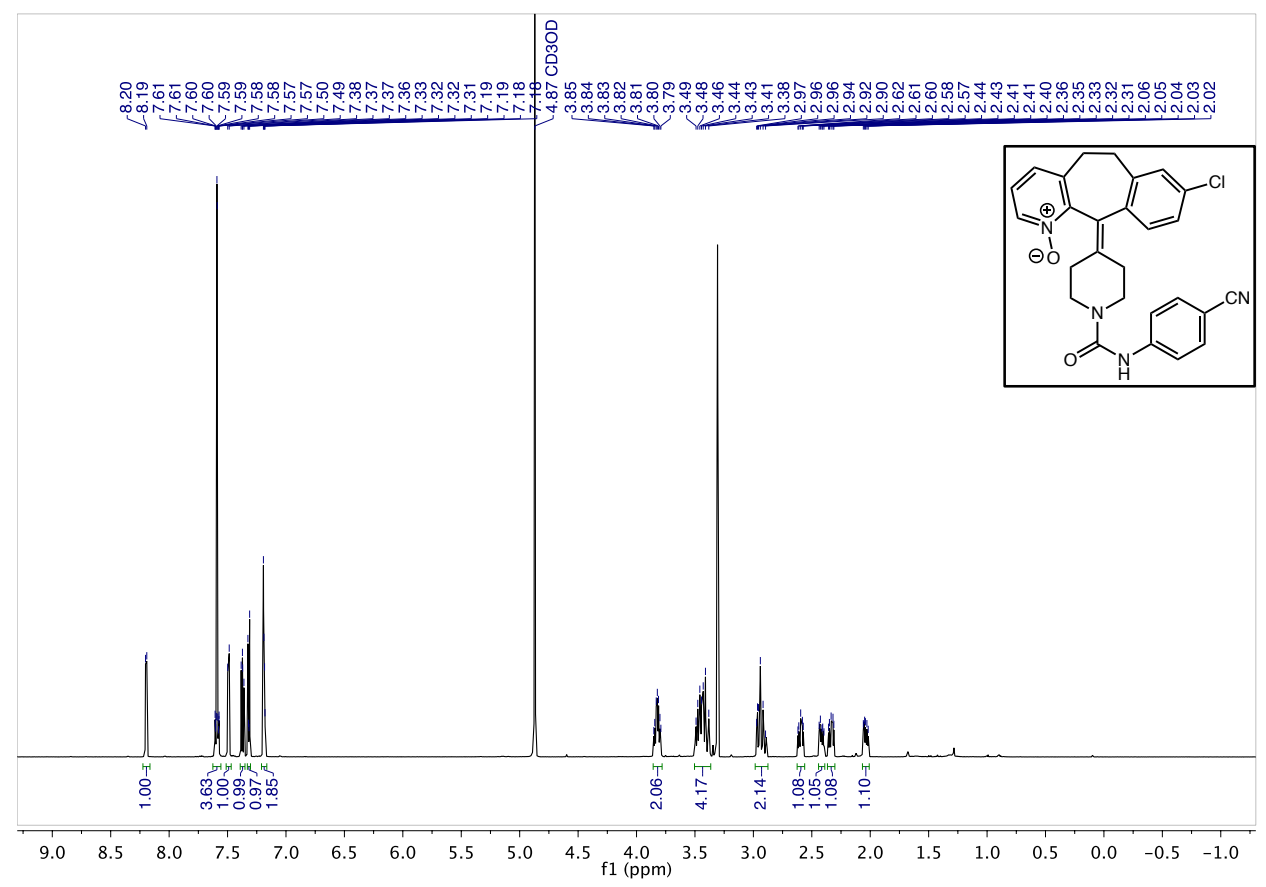

${ }^{13} \mathrm{C}$ NMR $\left(151 \mathrm{MHz}, \mathrm{CD}_{3} \mathrm{OD}\right)-\mathbf{6 f}$

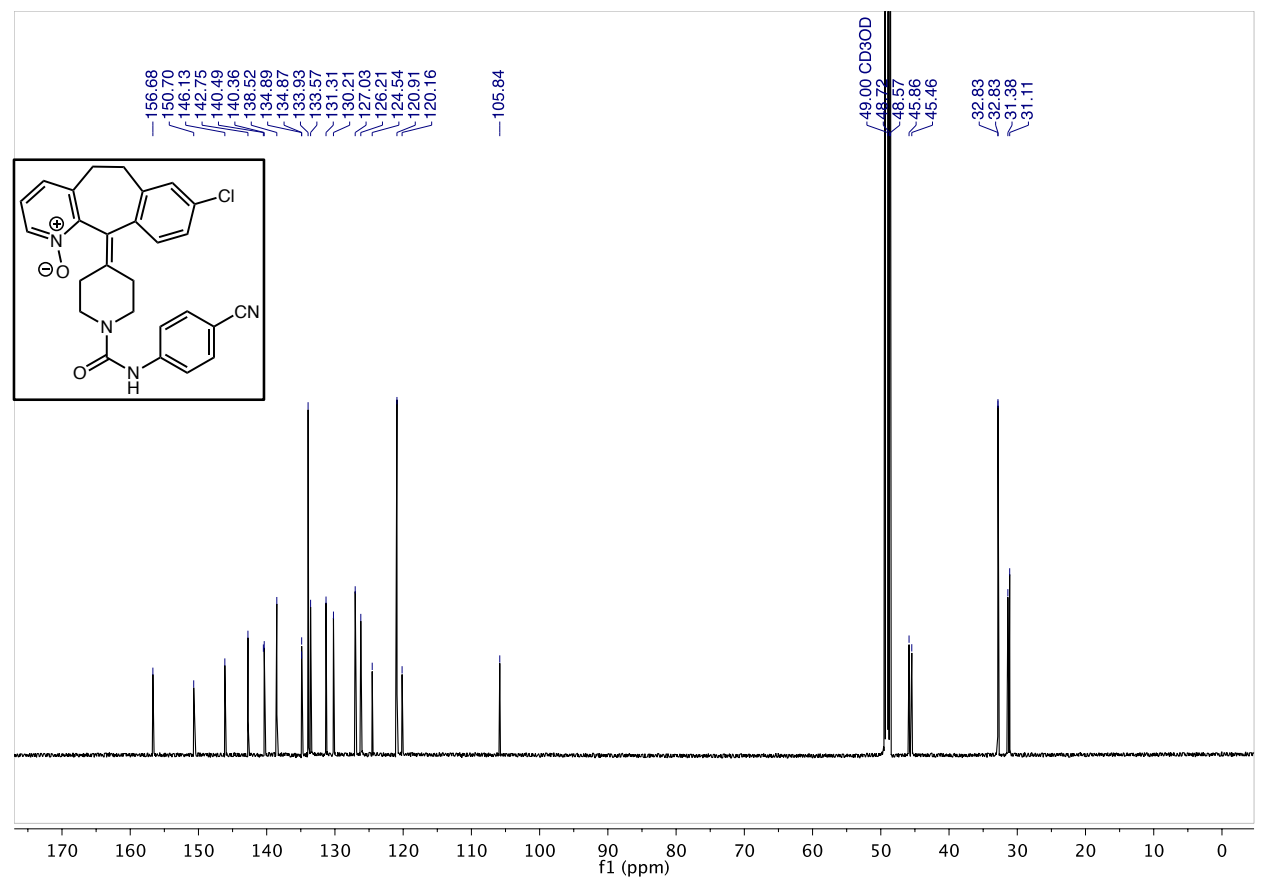


${ }^{1} \mathrm{H}$ NMR $\left(600 \mathrm{MHz}, \mathrm{CDCl}_{3}\right)-\mathbf{1 g}$

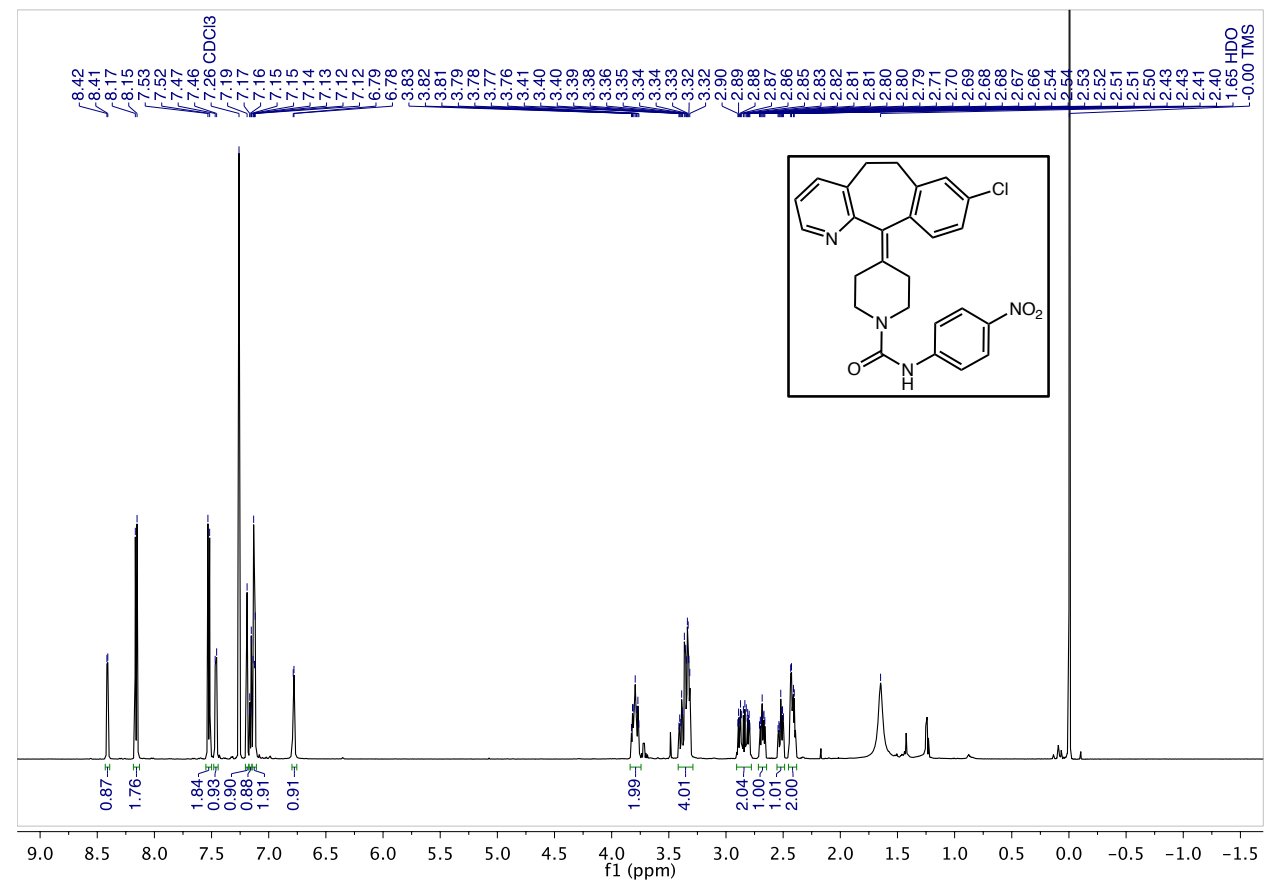

${ }^{13} \mathrm{C} \mathrm{NMR}\left(151 \mathrm{MHz}, \mathrm{CDCl}_{3}\right)-\mathbf{1 g}$

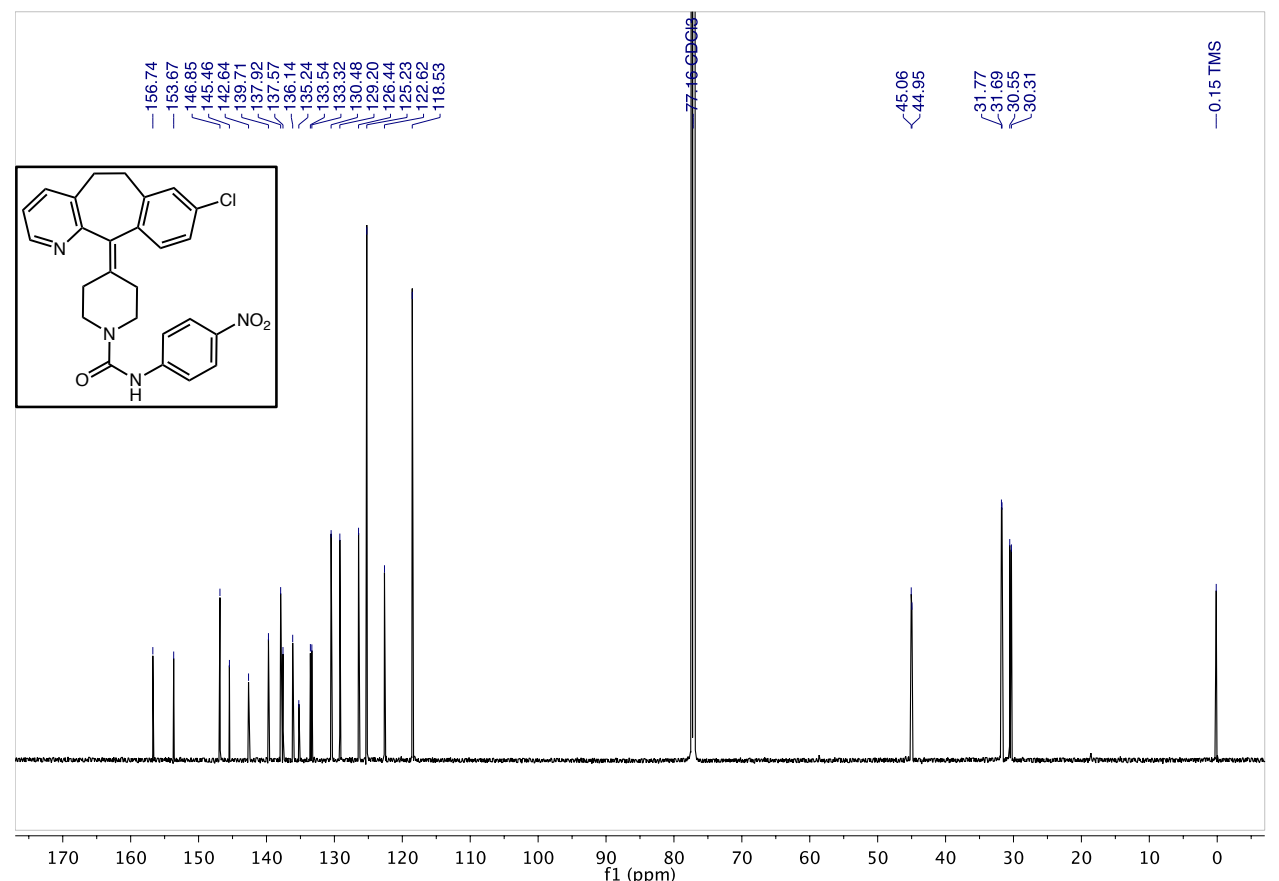


${ }^{1} \mathrm{H}$ NMR $\left(500 \mathrm{MHz}, \mathrm{CDCl}_{3}\right)-\mathbf{6 g}$

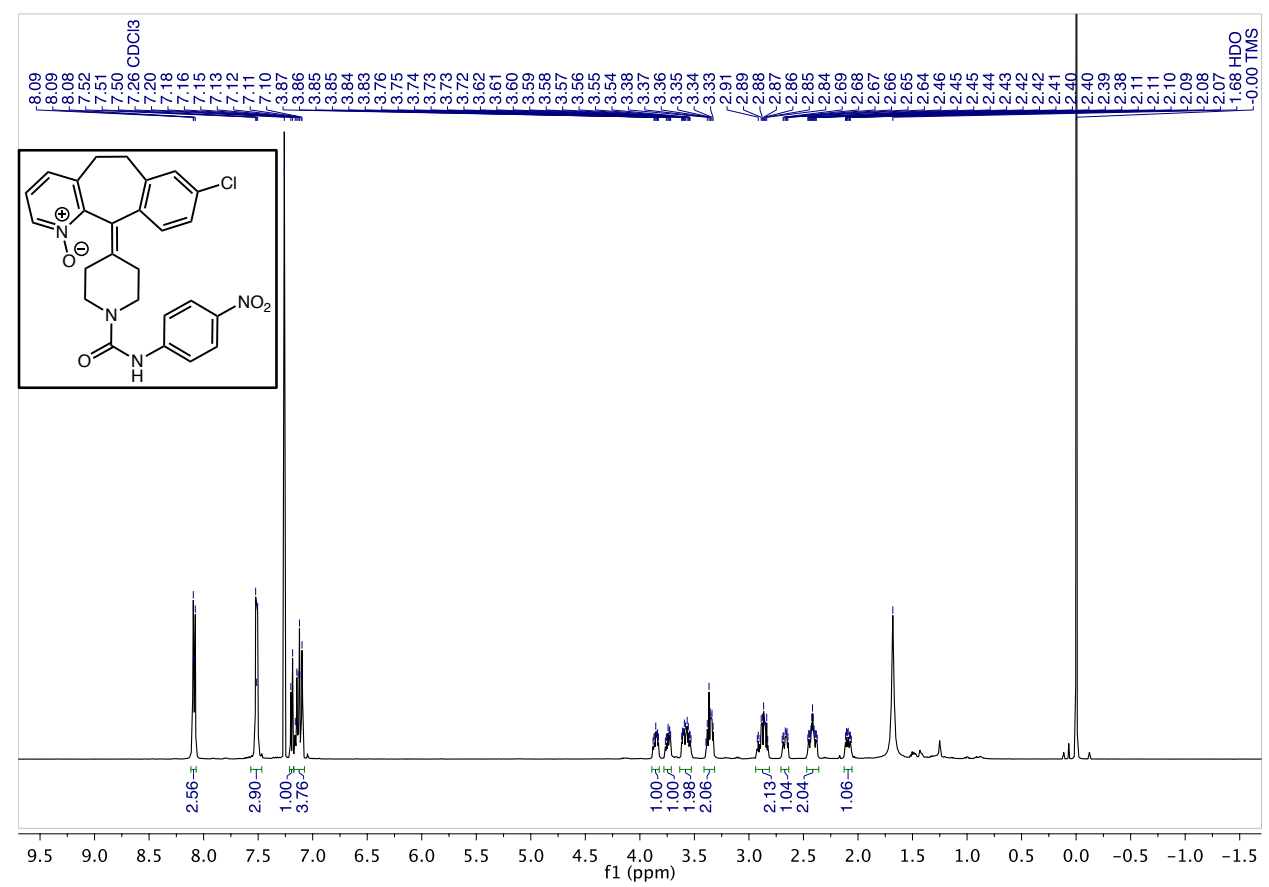

${ }^{13} \mathrm{C} \operatorname{NMR}\left(126 \mathrm{MHz}, \mathrm{CDCl}_{3}\right)-6 \mathrm{~g}$
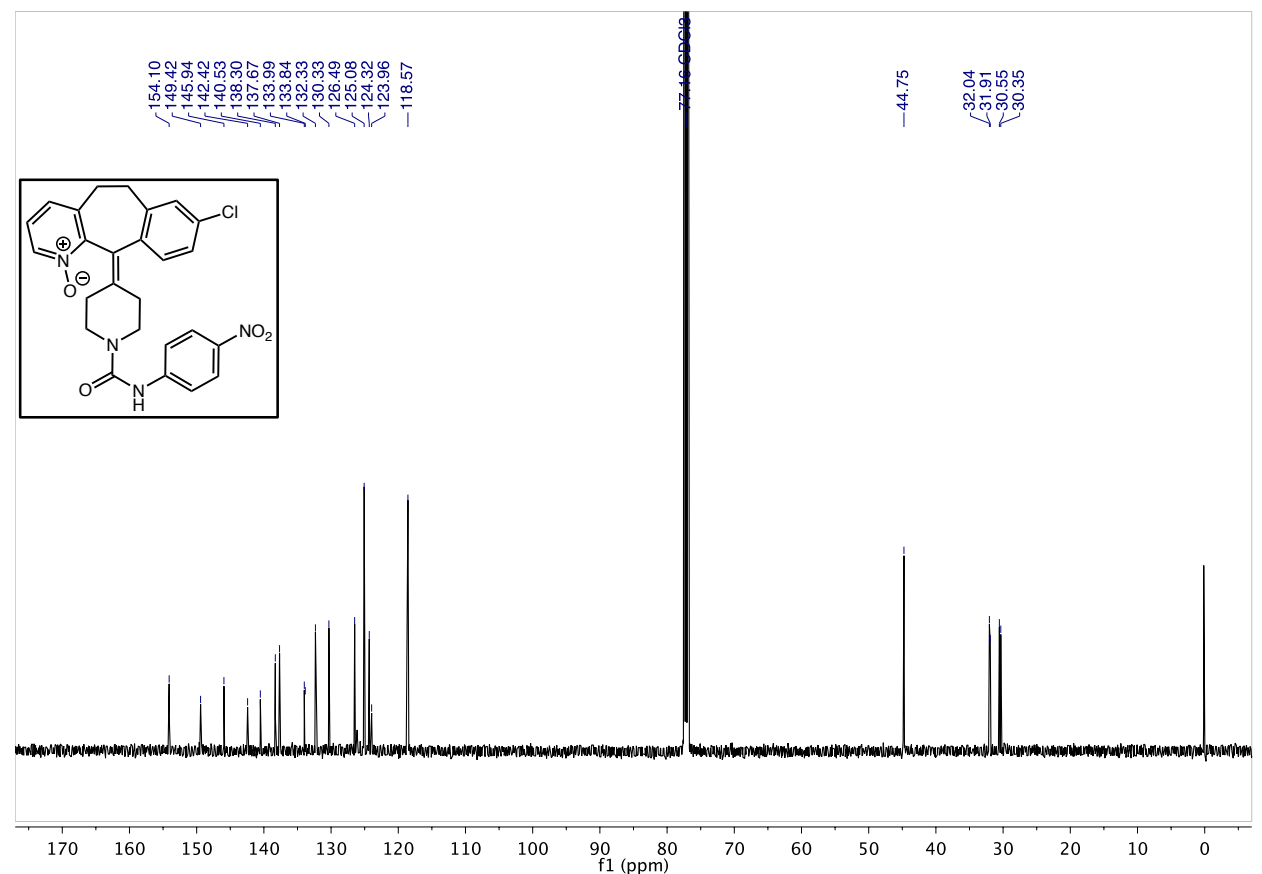
${ }^{1} \mathrm{H} \operatorname{NMR}\left(500 \mathrm{MHz}, \mathrm{CD}_{3} \mathrm{OD}\right)-\mathbf{1 h}$

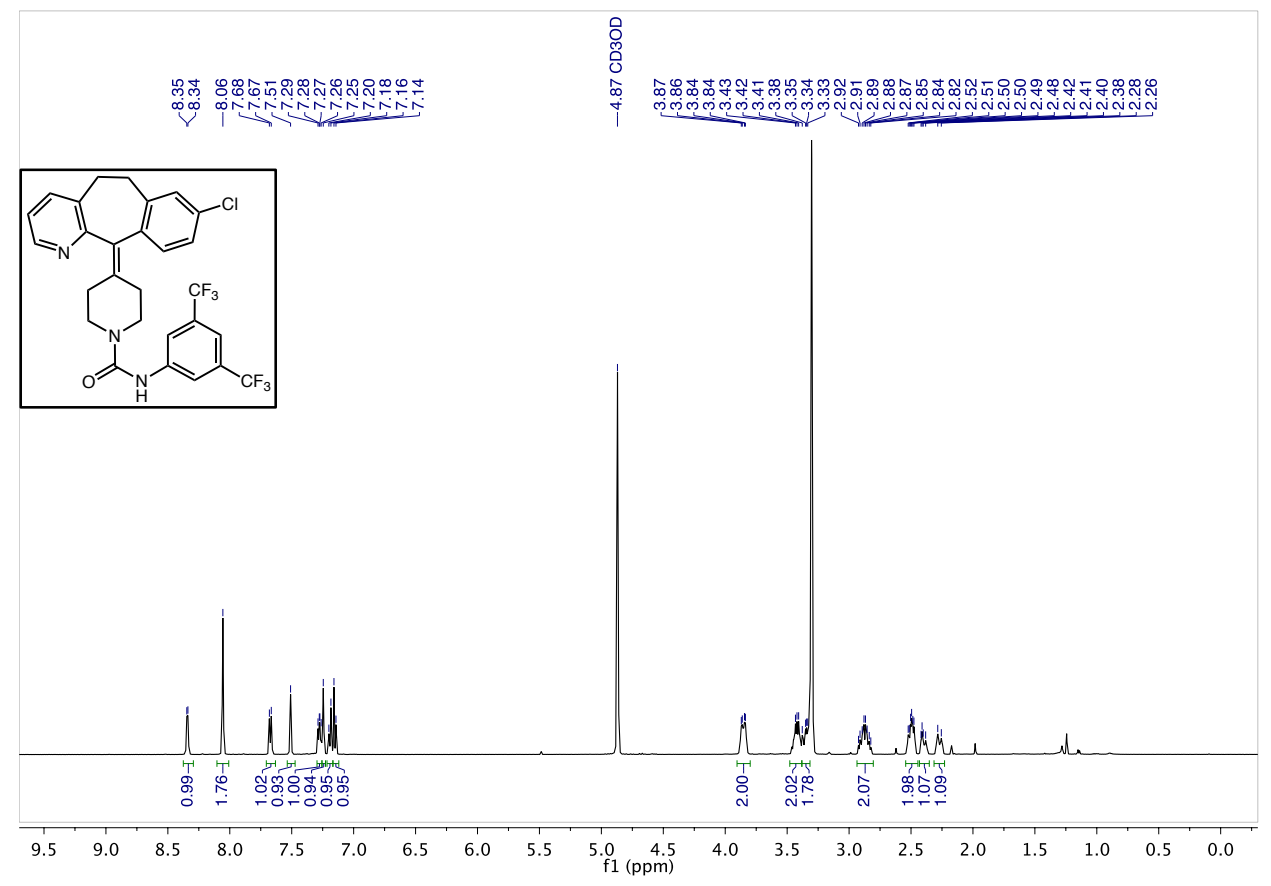

${ }^{13} \mathrm{C}$ NMR $\left(151 \mathrm{MHz}, \mathrm{CD}_{3} \mathrm{OD}\right)-\mathbf{1 h}$

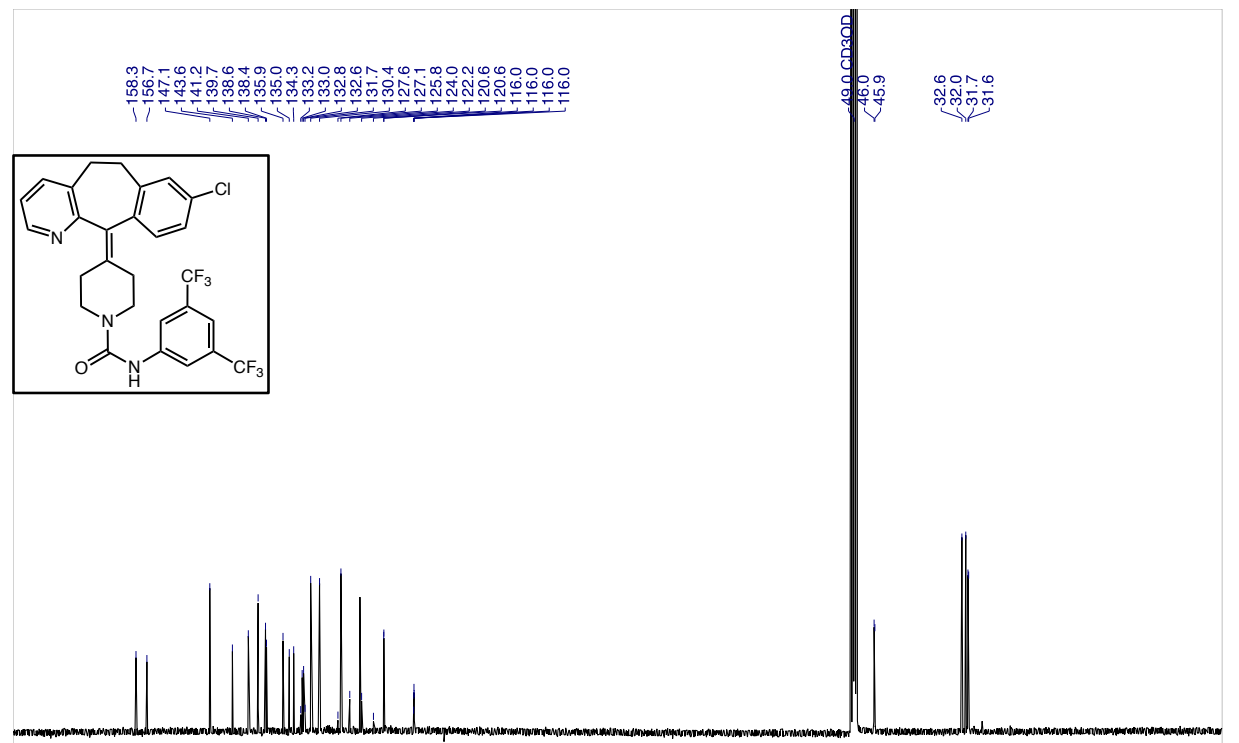

$\begin{array}{llllllllllllllllll}170 & 160 & 150 & 140 & 130 & 120 & 110 & 100 & \underset{9}{90} \underset{(\mathrm{ppm})}{80} & 70 & 60 & 50 & 40 & 30 & 20 & 10 & 0\end{array}$ 
${ }^{19} \mathrm{~F}$ NMR $\left(470 \mathrm{MHz}, \mathrm{CD}_{3} \mathrm{OD}\right)-\mathbf{1 h}$

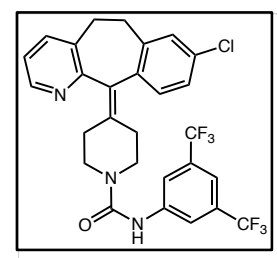

迎

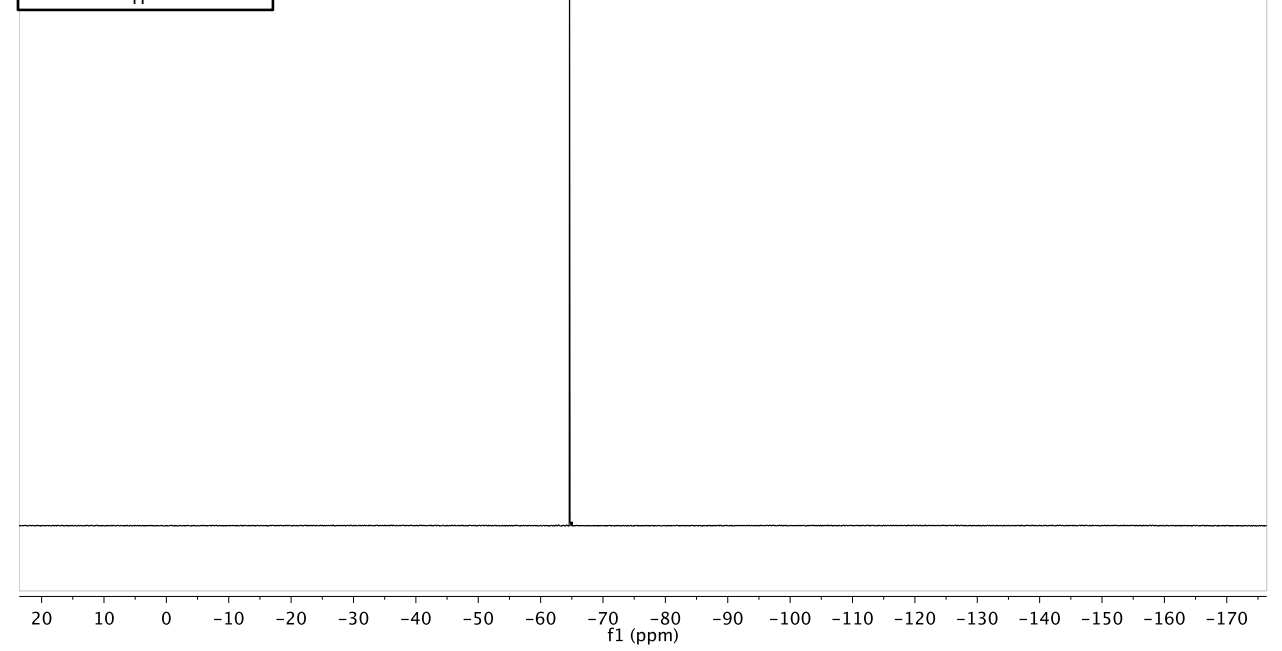

${ }^{1} \mathrm{H} \mathrm{NMR}\left(600 \mathrm{MHz}, \mathrm{CDCl}_{3}\right)-\mathbf{6 h}$

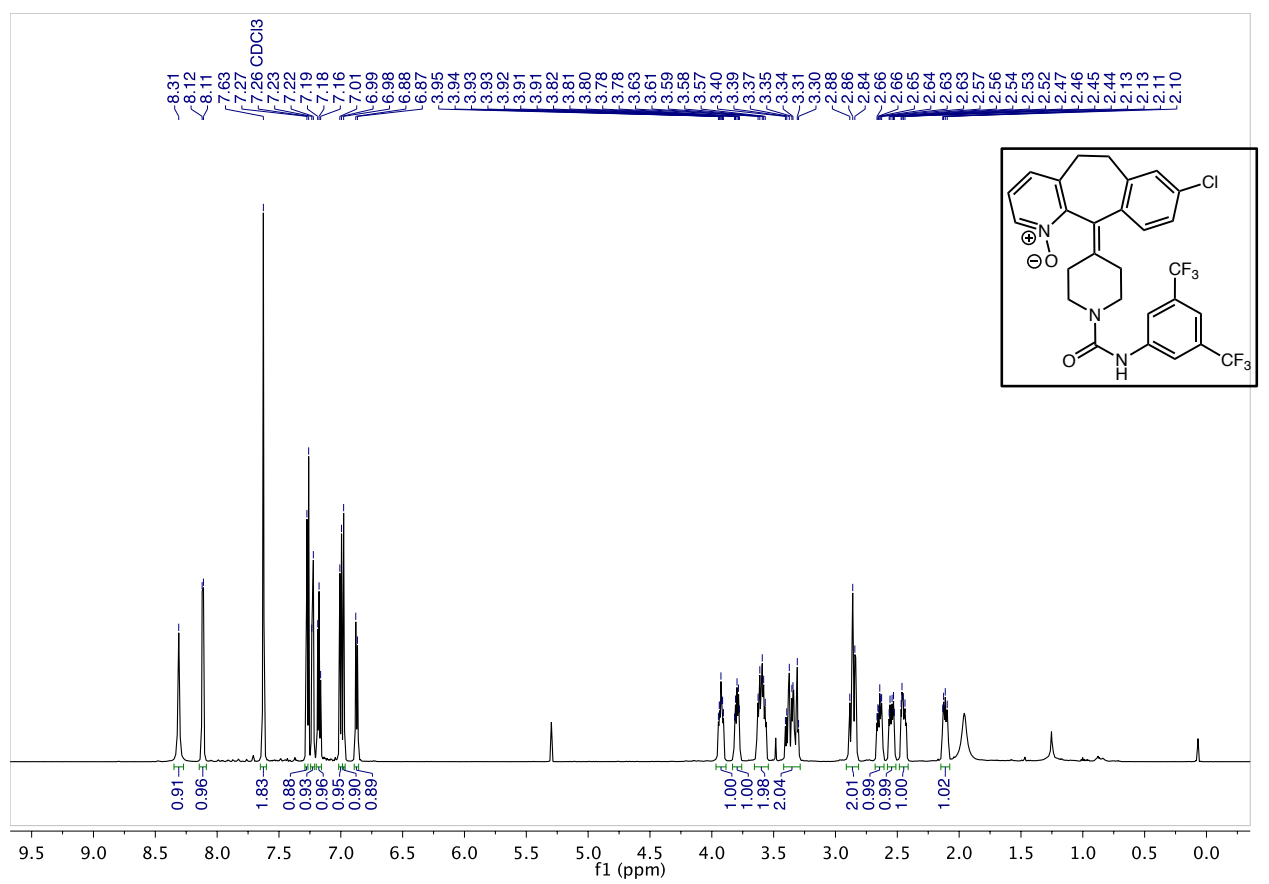


${ }^{13} \mathrm{C} \mathrm{NMR}\left(151 \mathrm{MHz}, \mathrm{CDCl}_{3}\right)-\mathbf{6 h}$

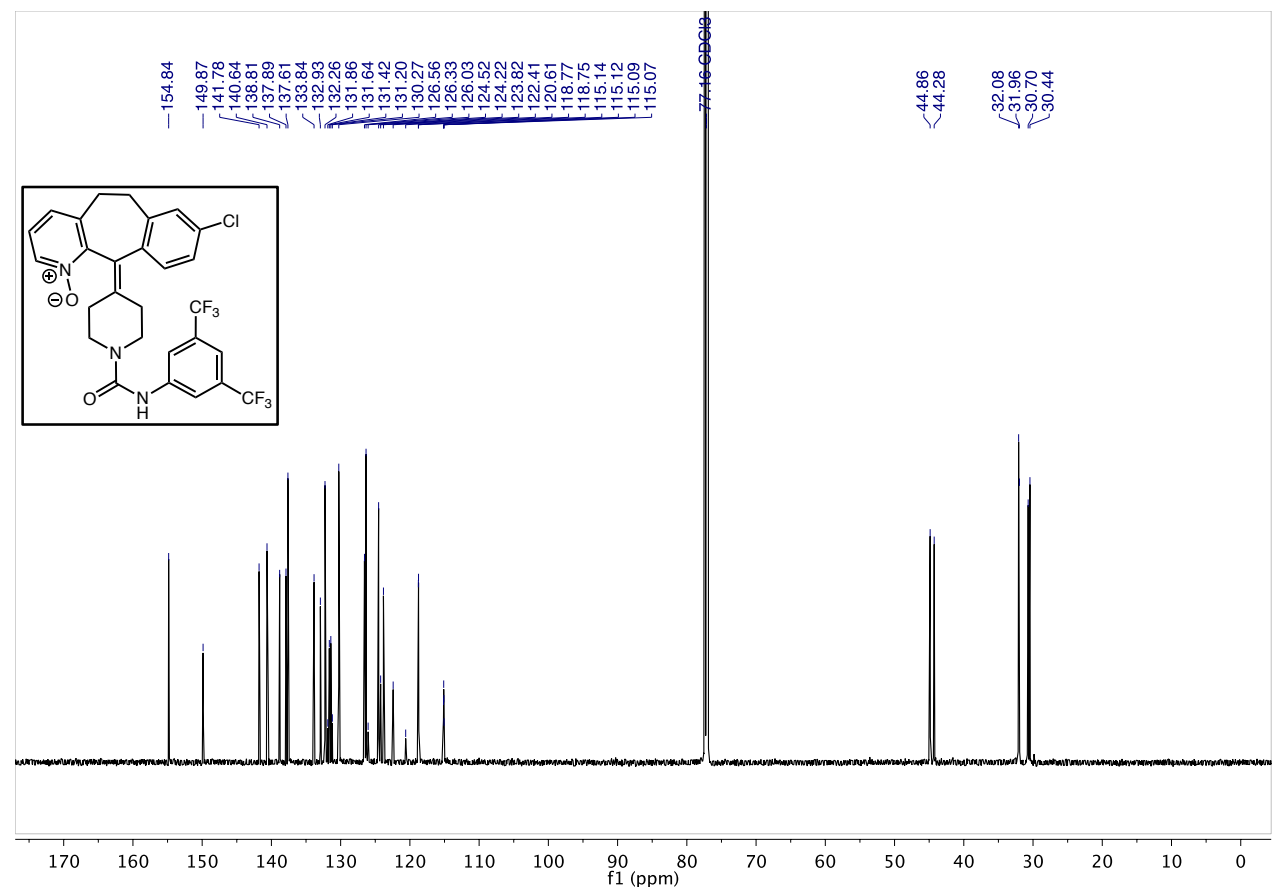

${ }^{19} \mathrm{~F}$ NMR $\left(471 \mathrm{MHz}, \mathrm{CDCl}_{3}\right)-\mathbf{6 h}$

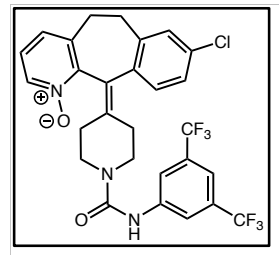


${ }^{1} \mathrm{H} \mathrm{NMR}\left(600 \mathrm{MHz}, \mathrm{CD}_{3} \mathrm{OD}\right)-\mathbf{7 h}$
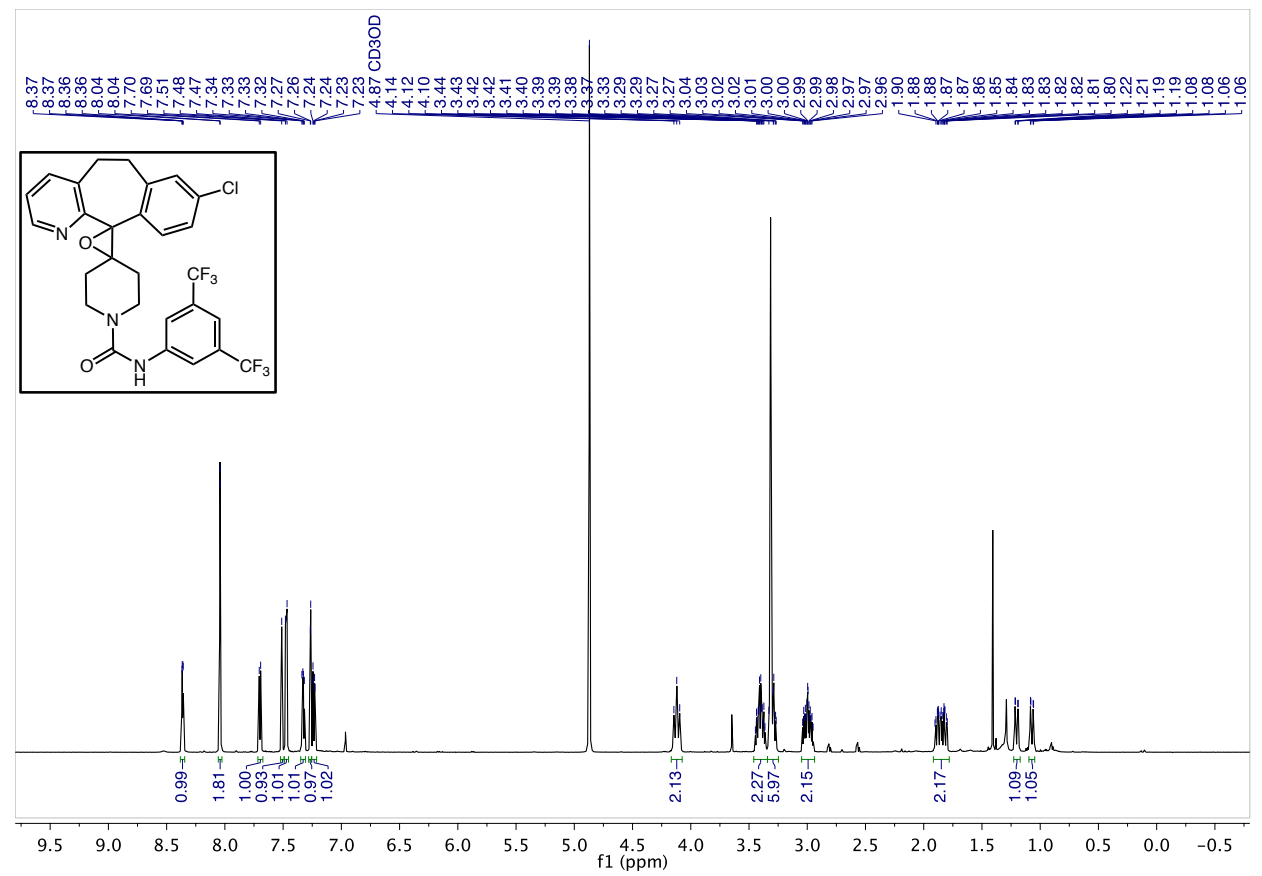

${ }^{13} \mathrm{C}$ NMR $\left(151 \mathrm{MHz}, \mathrm{CD}_{3} \mathrm{OD}\right)-\mathbf{7 h}$

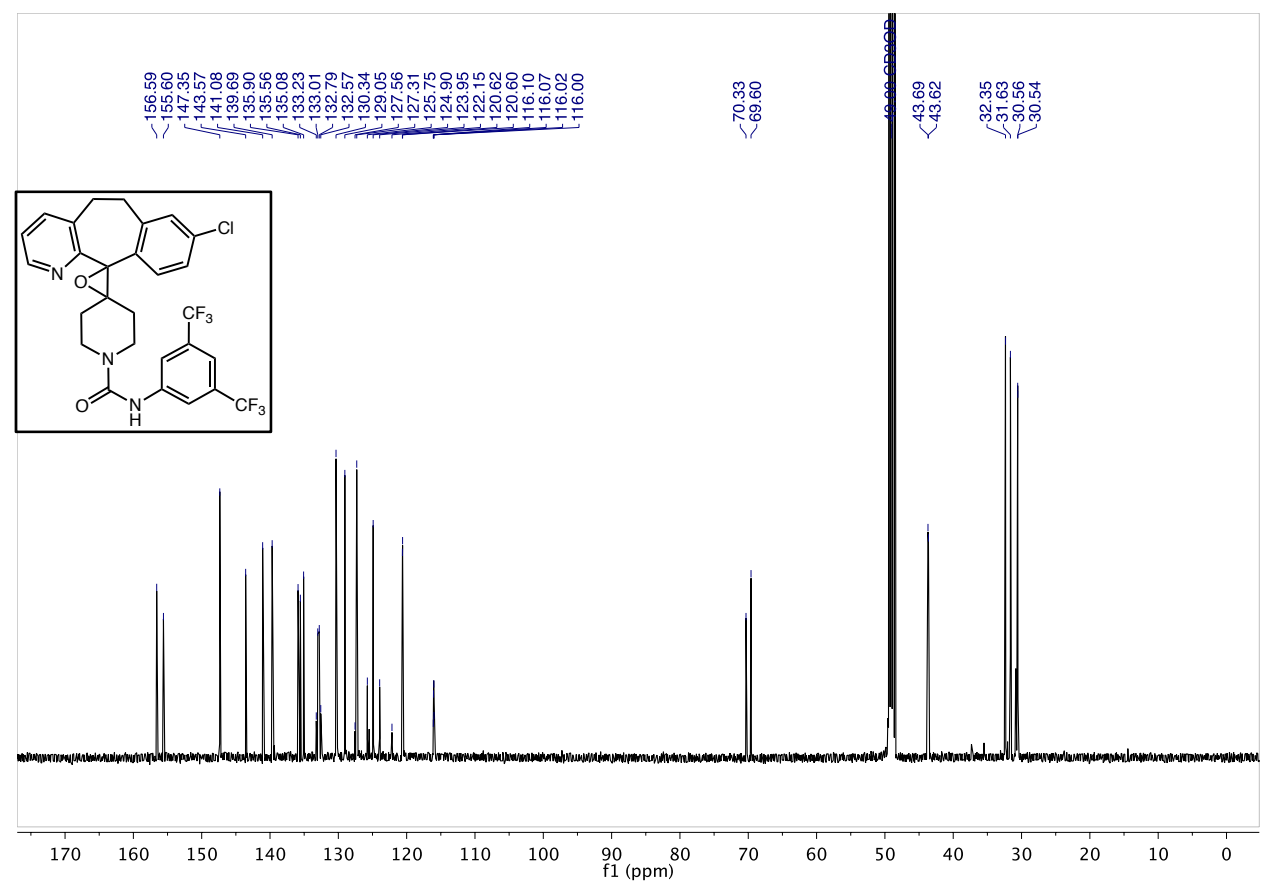


${ }^{19} \mathrm{~F}$ NMR $\left(471 \mathrm{MHz}, \mathrm{CD}_{3} \mathrm{OD}\right)-\mathbf{7 h}$
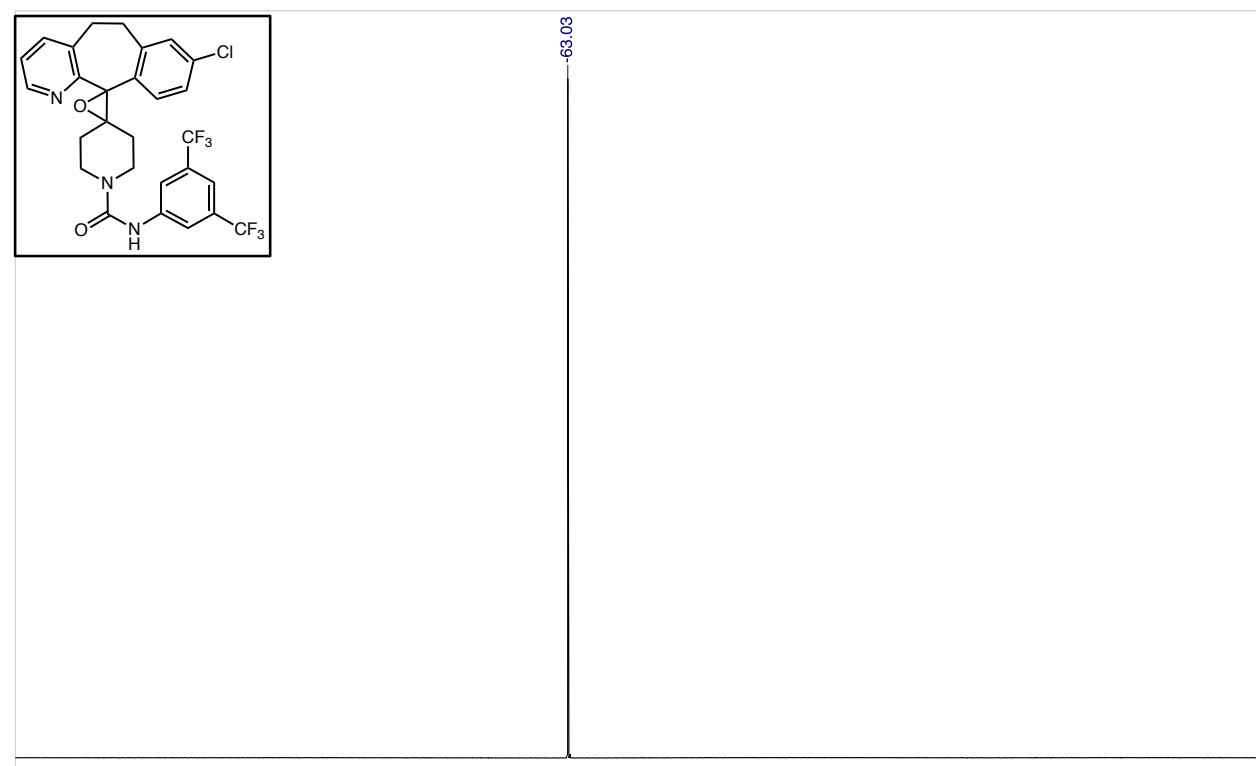

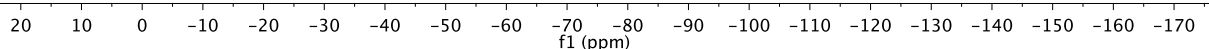

${ }^{1} \mathrm{H}$ NMR $\left(600 \mathrm{MHz}, \mathrm{CD}_{3} \mathrm{OD}\right)-\mathbf{8 h}$

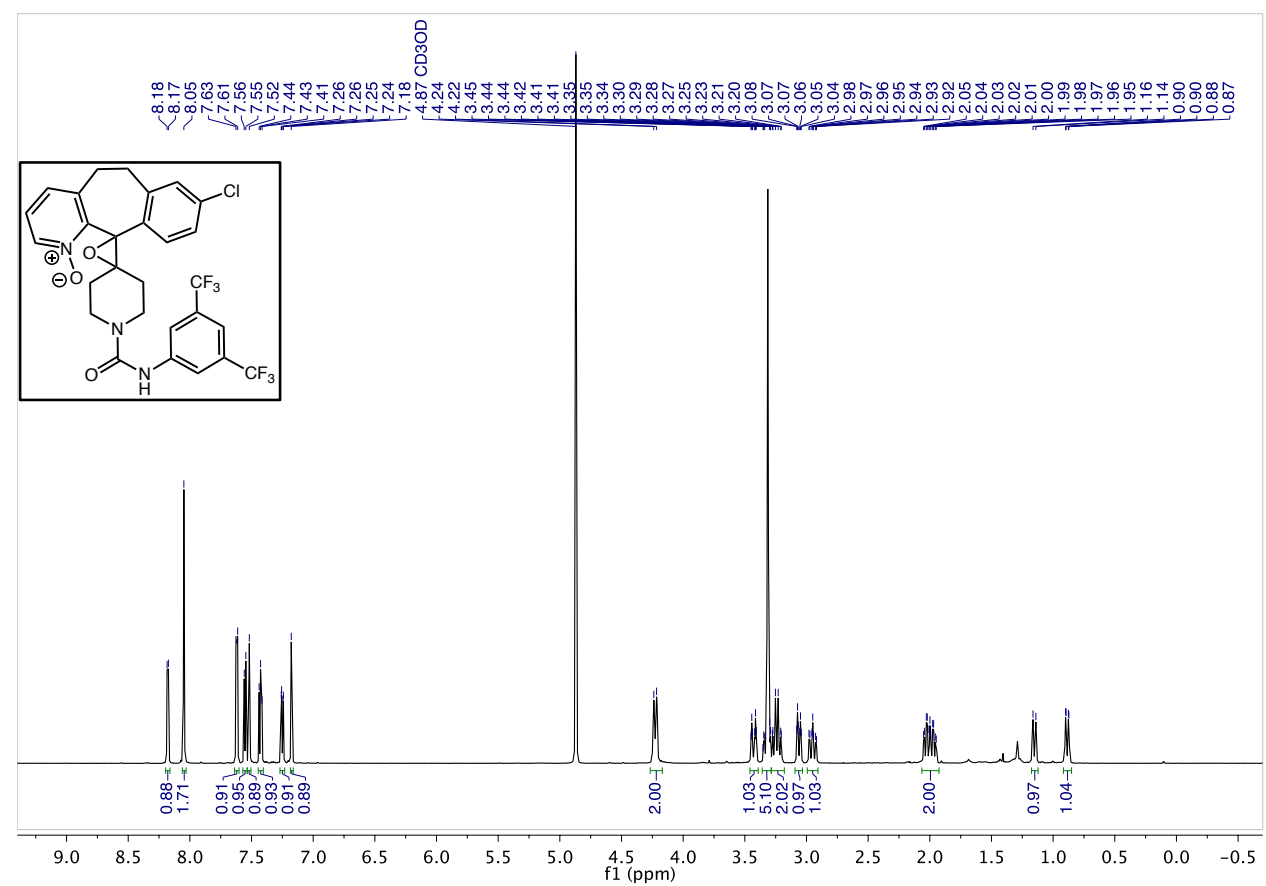


${ }^{13} \mathrm{C}$ NMR $\left(151 \mathrm{MHz}, \mathrm{CD}_{3} \mathrm{OD}\right)-\mathbf{8 h}$

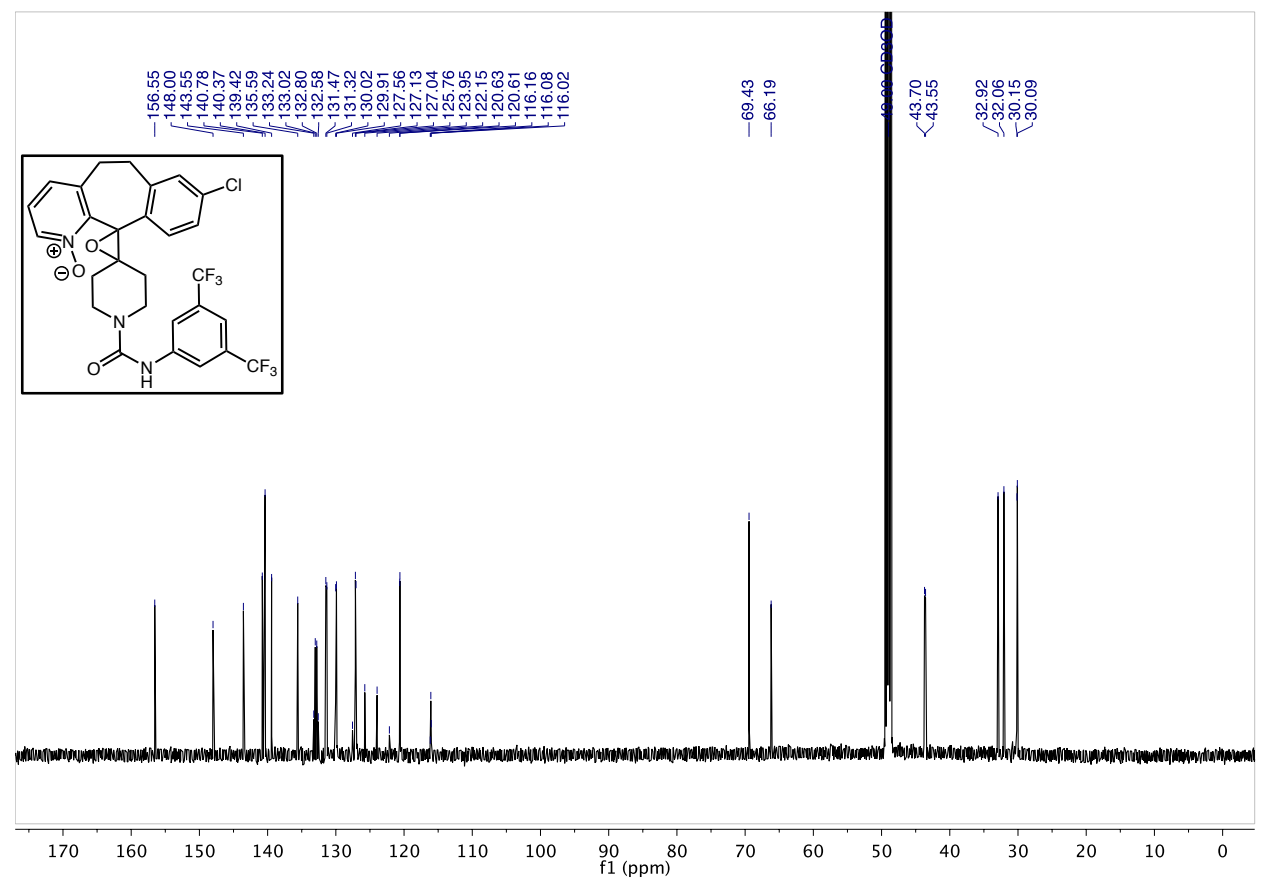

${ }^{19} \mathrm{~F}$ NMR $\left(471 \mathrm{MHz}, \mathrm{CD}_{3} \mathrm{OD}\right)-\mathbf{8 h}$
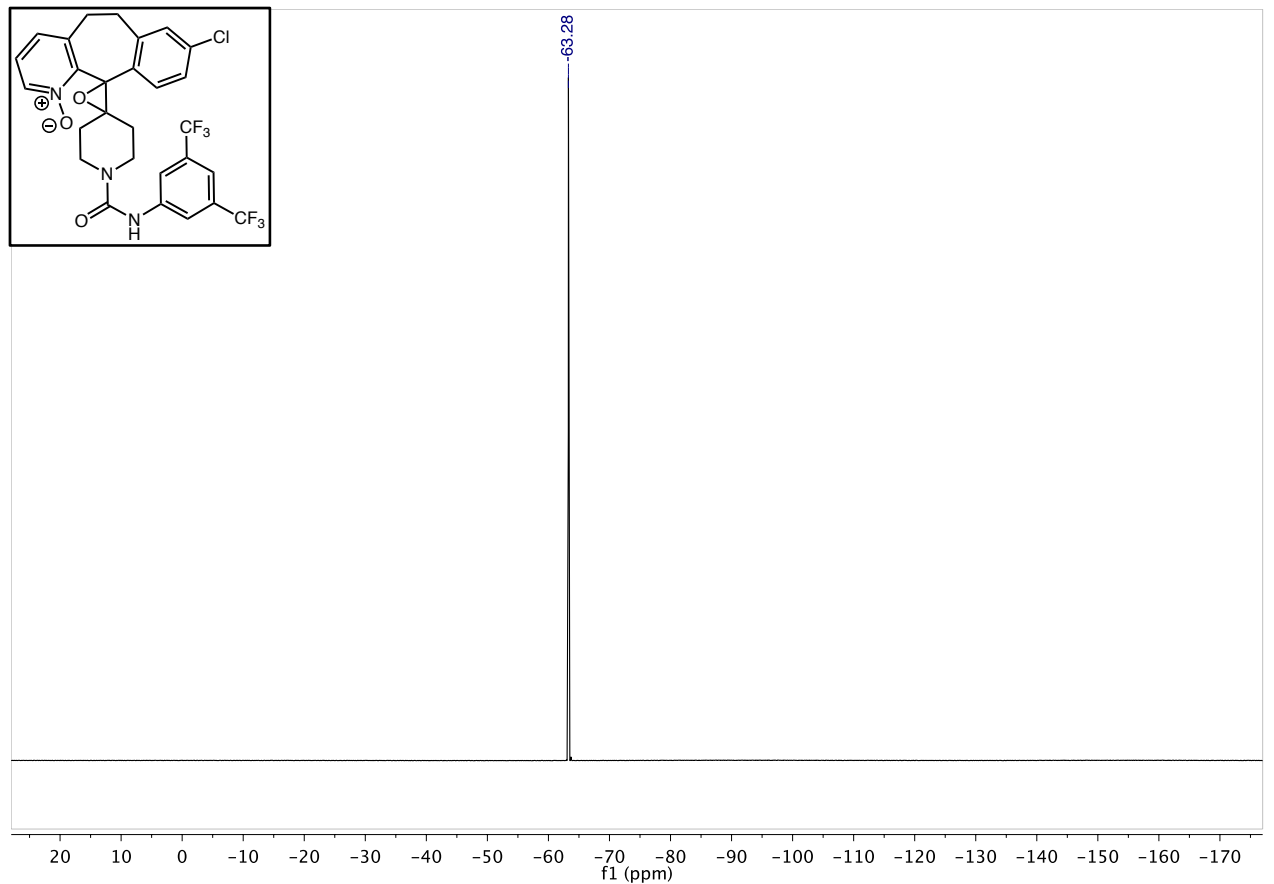
${ }^{1} \mathrm{H} \operatorname{NMR}\left(600 \mathrm{MHz}, \mathrm{CDCl}_{3}\right)-\mathbf{1 i}$

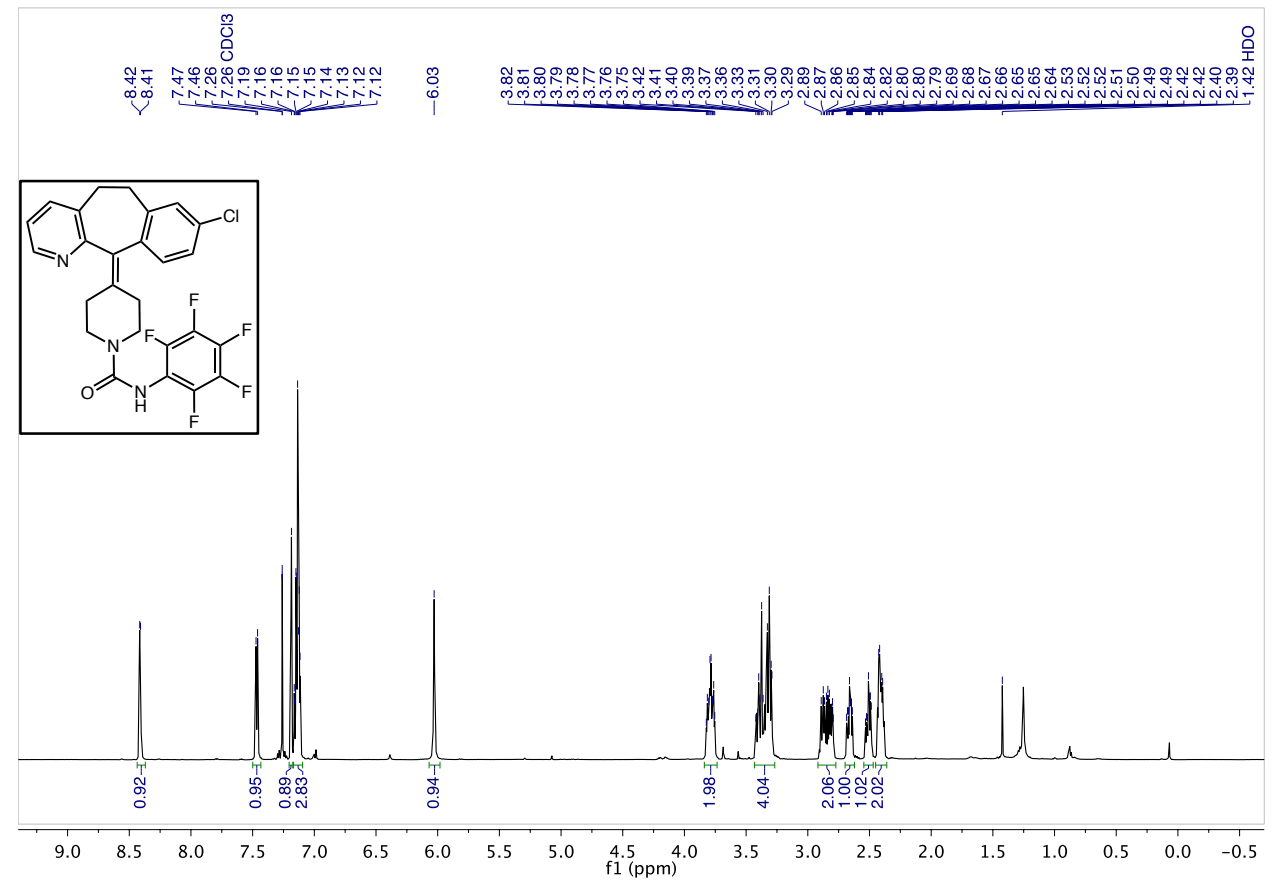

${ }^{13} \mathrm{C} \mathrm{NMR}\left(151 \mathrm{MHz}, \mathrm{CDCl}_{3}\right)-\mathbf{1 i}$

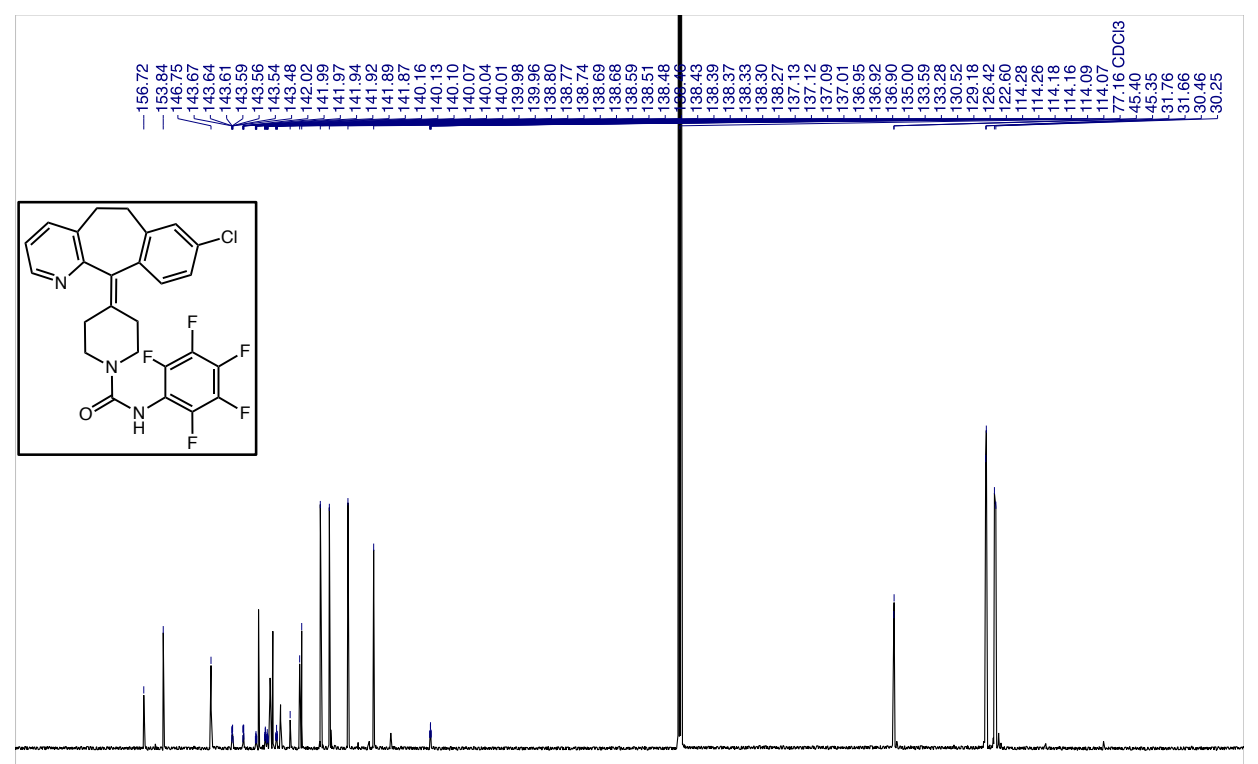

$\begin{array}{lllllllllllllllll}170 & 160 & 150 & 140 & 130 & 120 & 110 & 100 & \underset{f}{90}(\mathrm{ppm}) & 70 & 60 & 50 & 40 & 30 & 20 & 10 & 0\end{array}$ 
${ }^{19} \mathrm{~F}$ NMR $\left(471 \mathrm{MHz}, \mathrm{CDCl}_{3}\right)-\mathbf{1 i}$

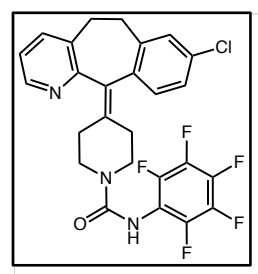

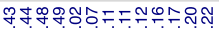

守守守品员

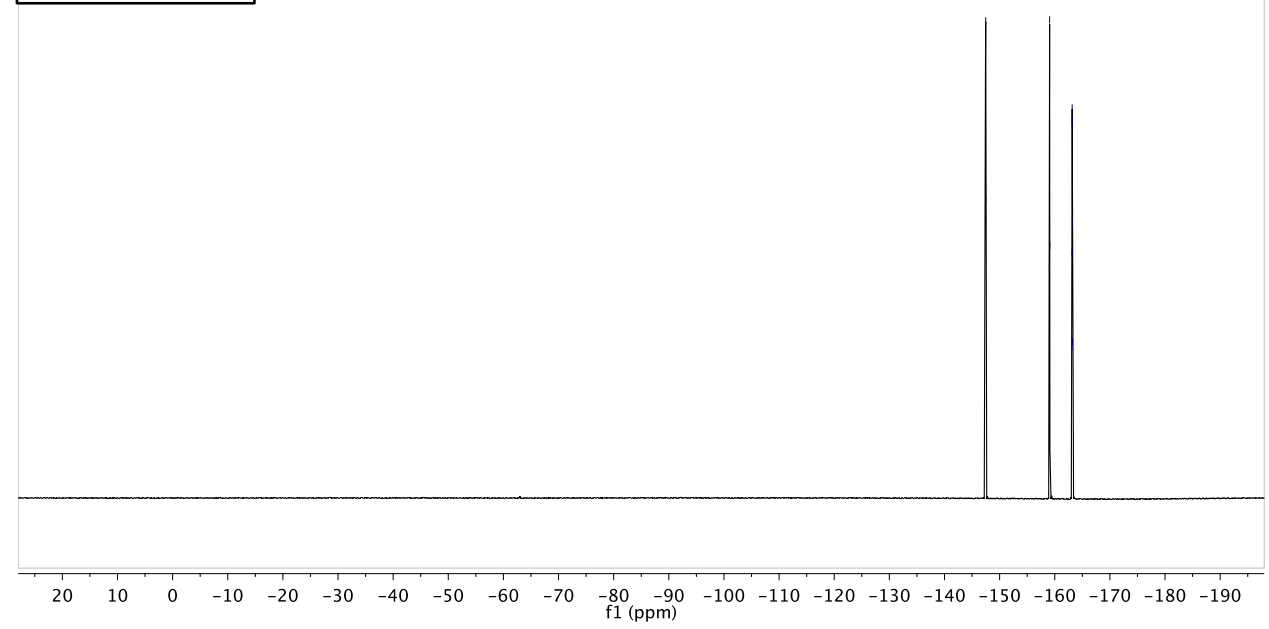

${ }^{1} \mathrm{H}$ NMR $\left(600 \mathrm{MHz}, \mathrm{CD}_{3} \mathrm{OD}\right)-6 \mathbf{i}$

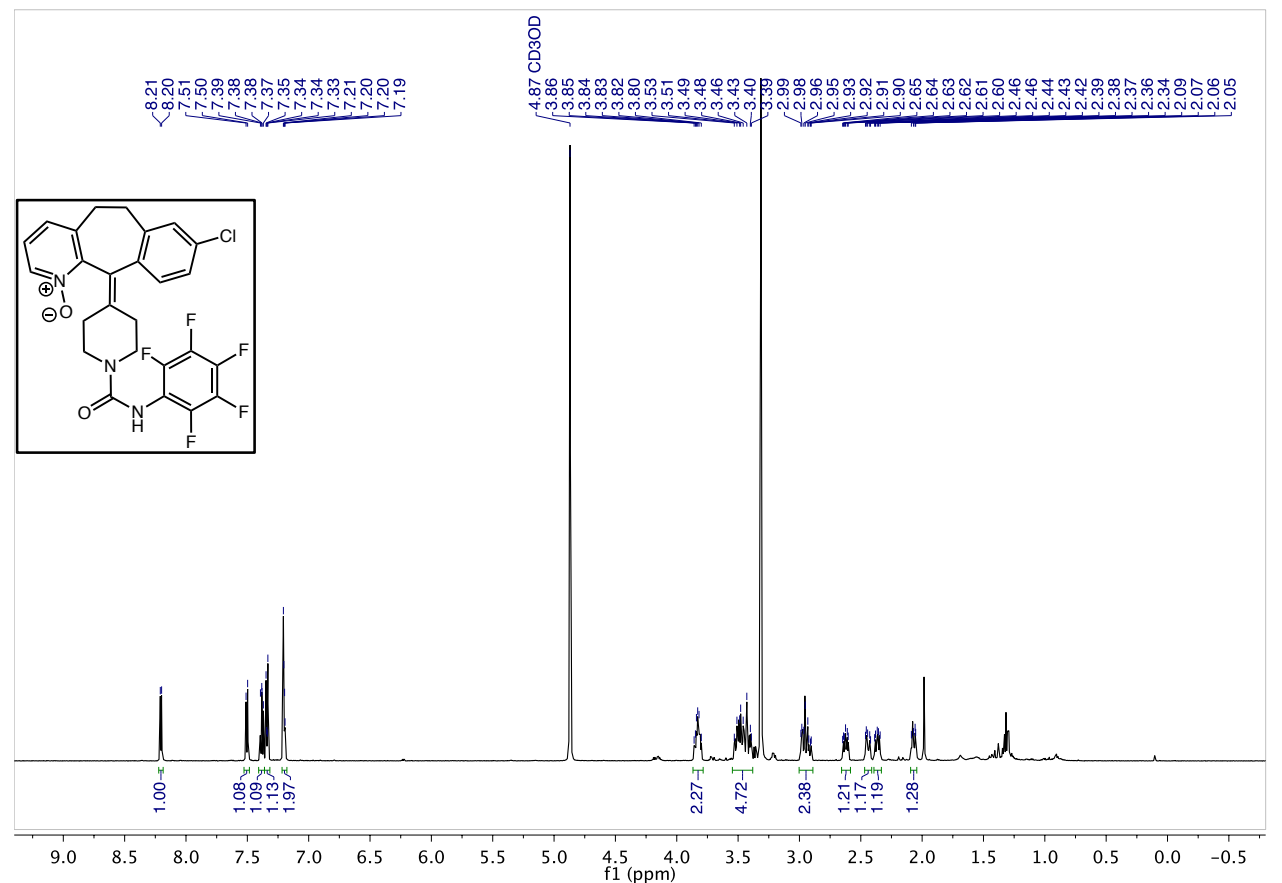


${ }^{13} \mathrm{C}$ NMR $\left(151 \mathrm{MHz}, \mathrm{CD}_{3} \mathrm{OD}\right)-\mathbf{6 i}$

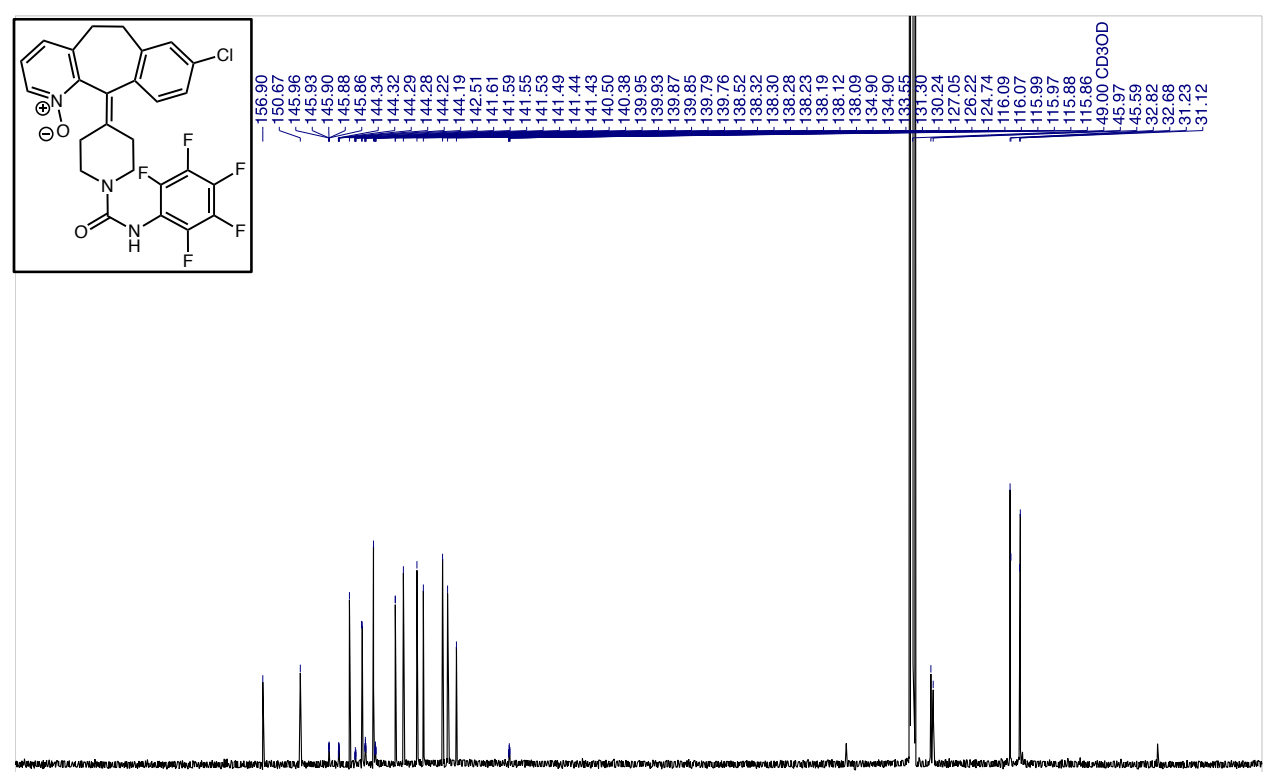

$\begin{array}{llllllllllllllllllll}190 & 180 & 170 & 160 & 150 & 140 & 130 & 120 & 110 & \begin{array}{r}100 \\ \text { f } 1(\mathrm{ppm})\end{array} & 80 & 70 & 60 & 50 & 40 & 30 & 20 & 10 & 0\end{array}$

${ }^{19} \mathrm{~F}$ NMR $\left(471 \mathrm{MHz}, \mathrm{CDCl}_{3}\right)-6 \mathbf{i}$

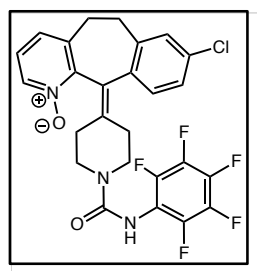

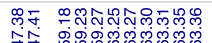

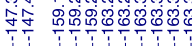

isizi

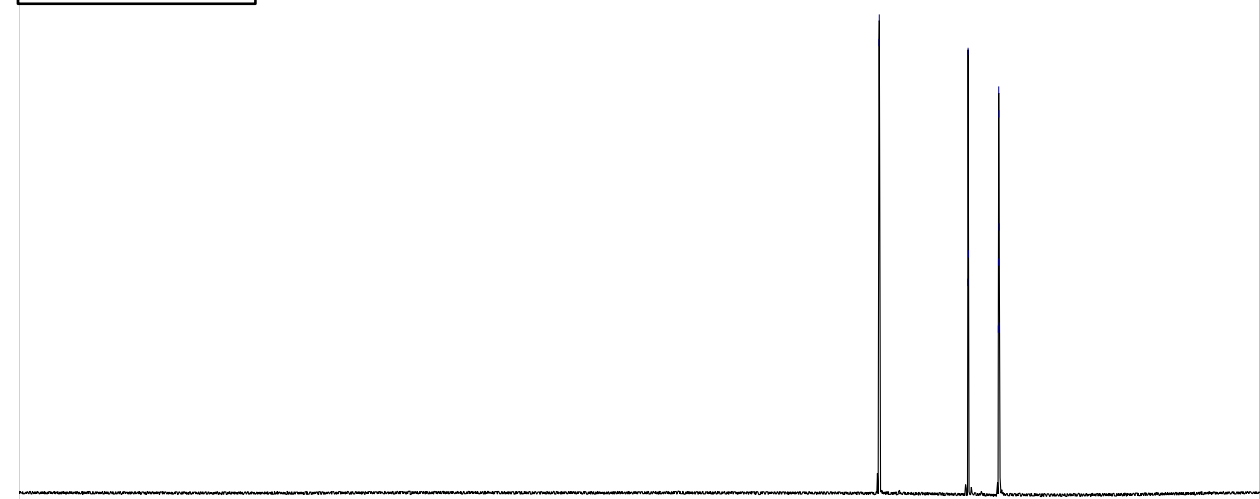

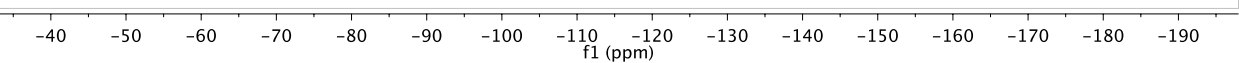


${ }^{1} \mathrm{H} \mathrm{NMR}\left(600 \mathrm{MHz}, \mathrm{CDCl}_{3}\right)-\mathbf{1 j}$

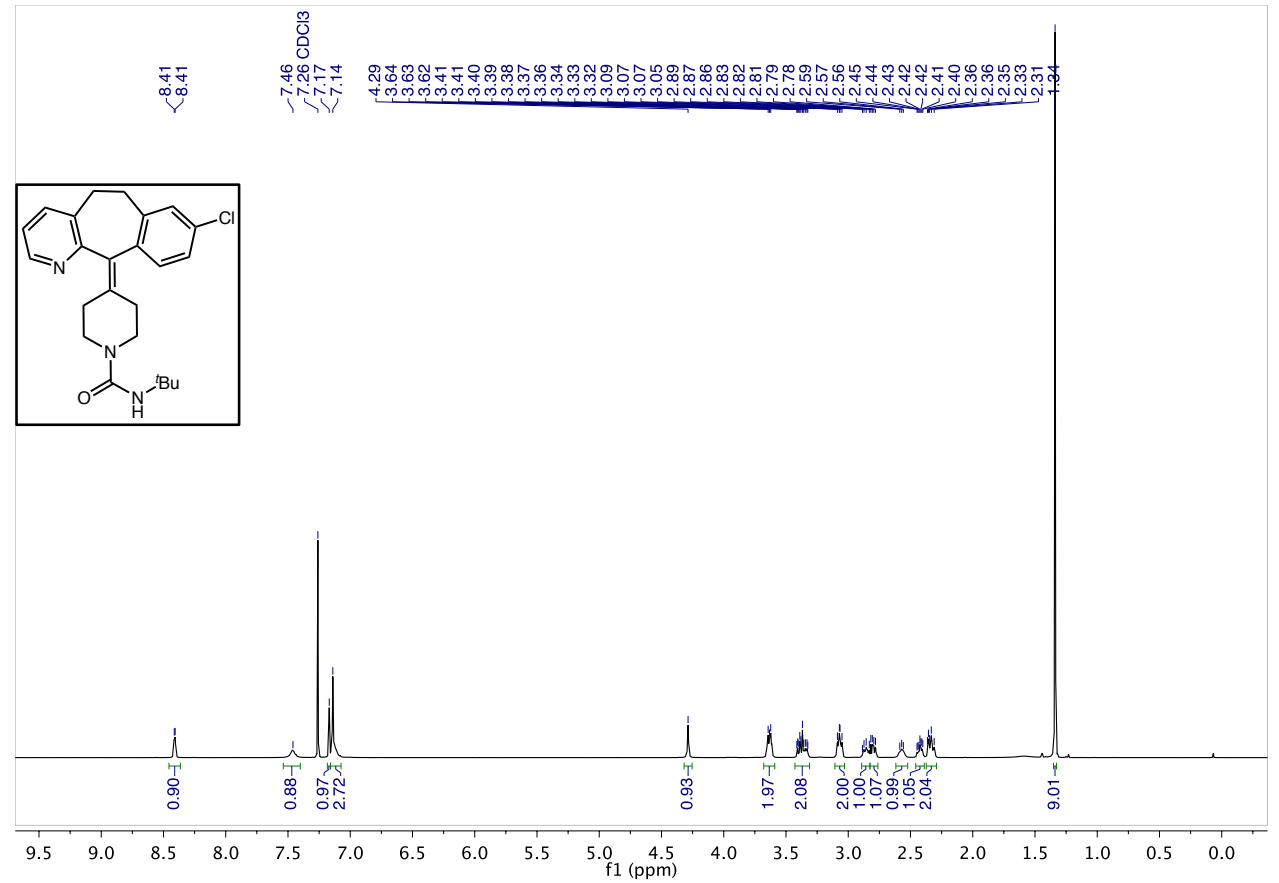

${ }^{13} \mathrm{C} \operatorname{NMR}\left(151 \mathrm{MHz}, \mathrm{CDCl}_{3}\right)-\mathbf{1 j}$

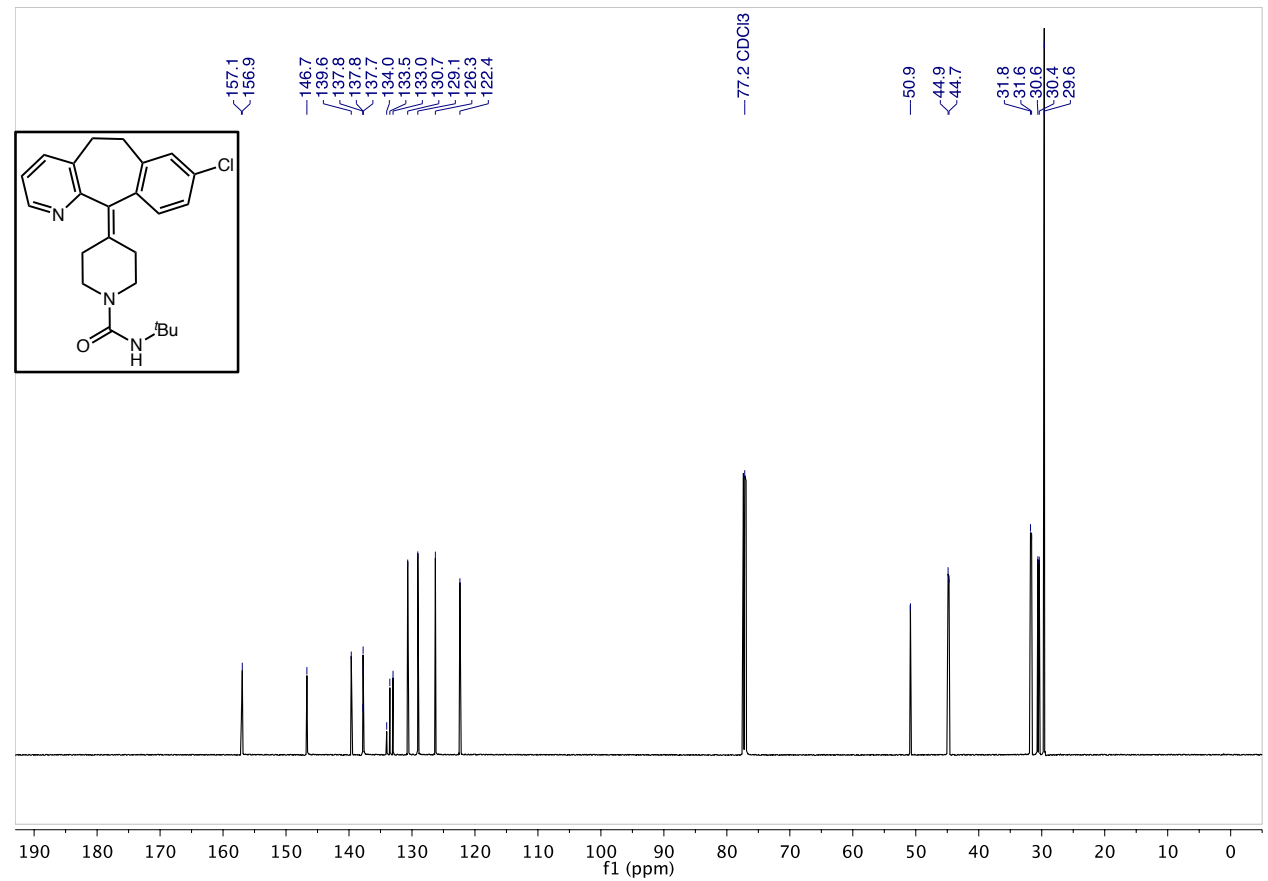


${ }^{1} \mathrm{H}$ NMR $\left(600 \mathrm{MHz}, \mathrm{CD}_{3} \mathrm{OD}\right)-\mathbf{6 j}$

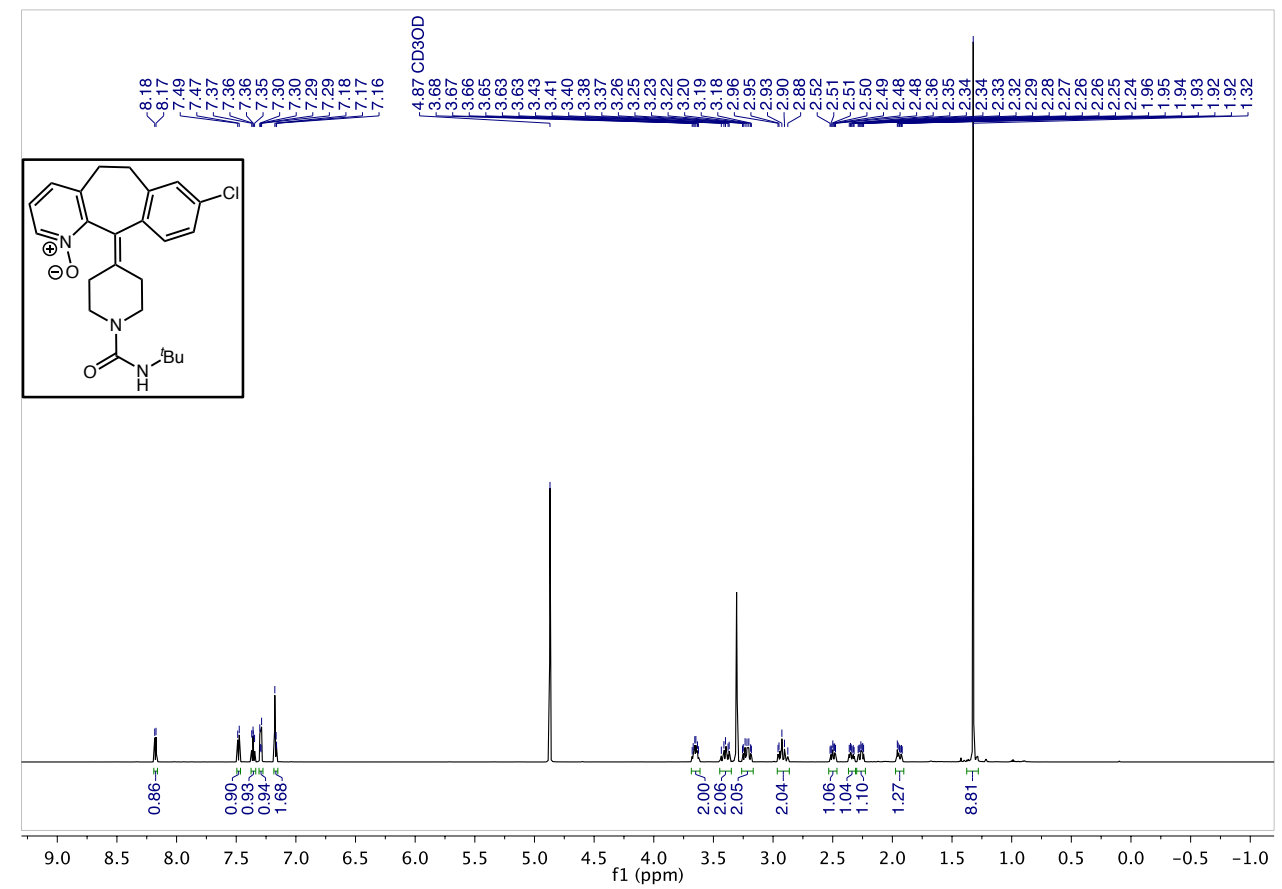

${ }^{13} \mathrm{C}$ NMR (151 MHz, $\left.\mathrm{CD}_{3} \mathrm{OD}\right)-\mathbf{6 j}$

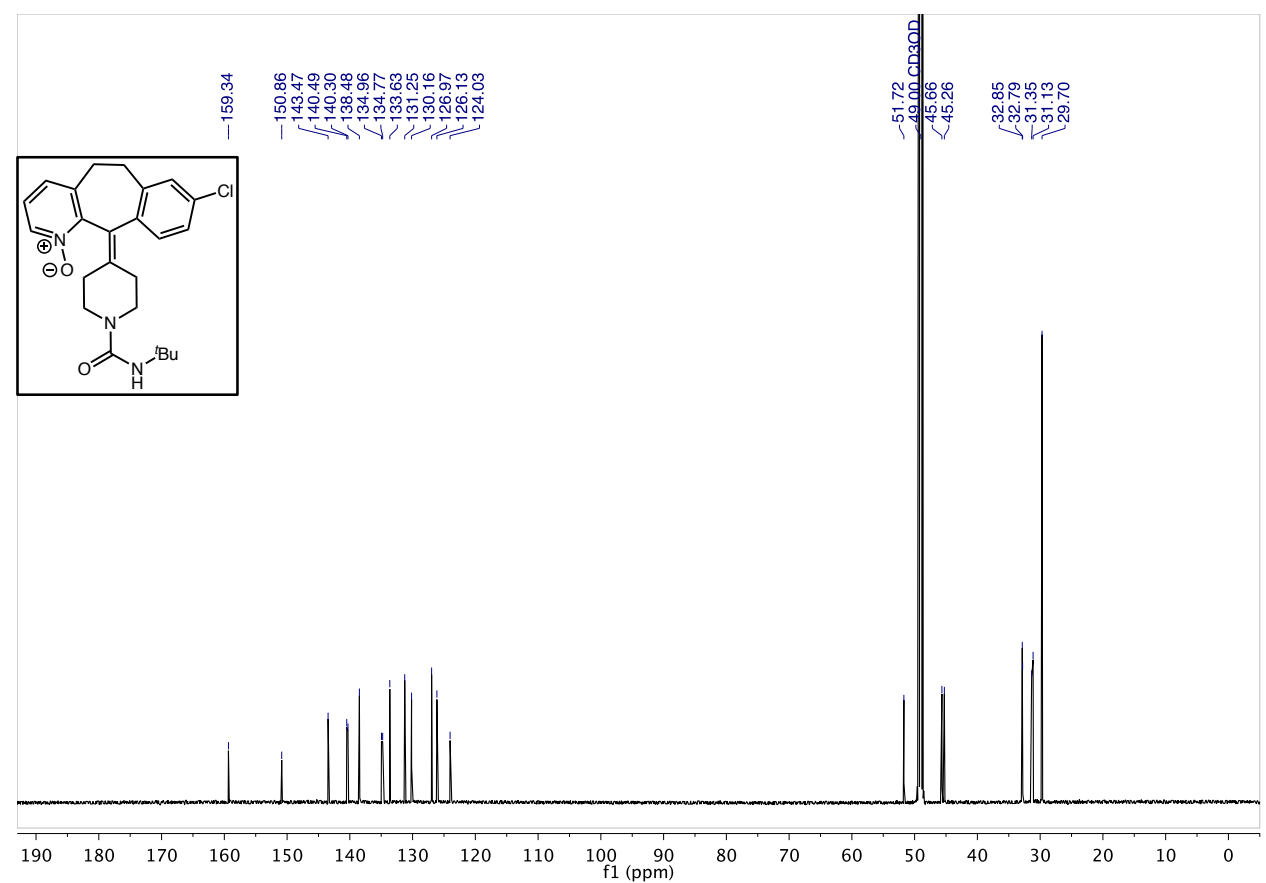


${ }^{1} \mathrm{H}$ NMR $\left(600 \mathrm{MHz}, \mathrm{CD}_{3} \mathrm{OD}\right)-\mathbf{1 k}$

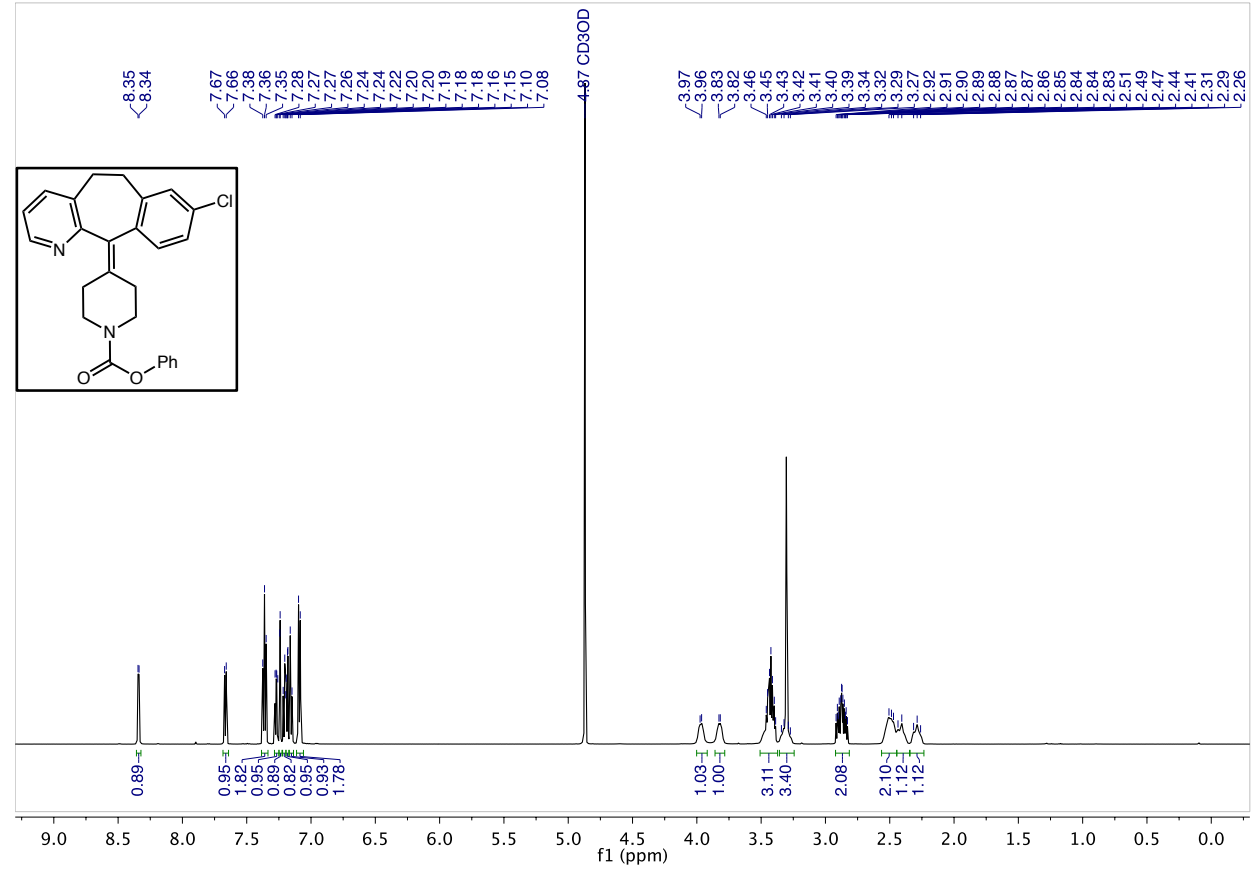

${ }^{13} \mathrm{C}$ NMR $\left(151 \mathrm{MHz}, \mathrm{CD}_{3} \mathrm{OD}\right)-\mathbf{1 k}$

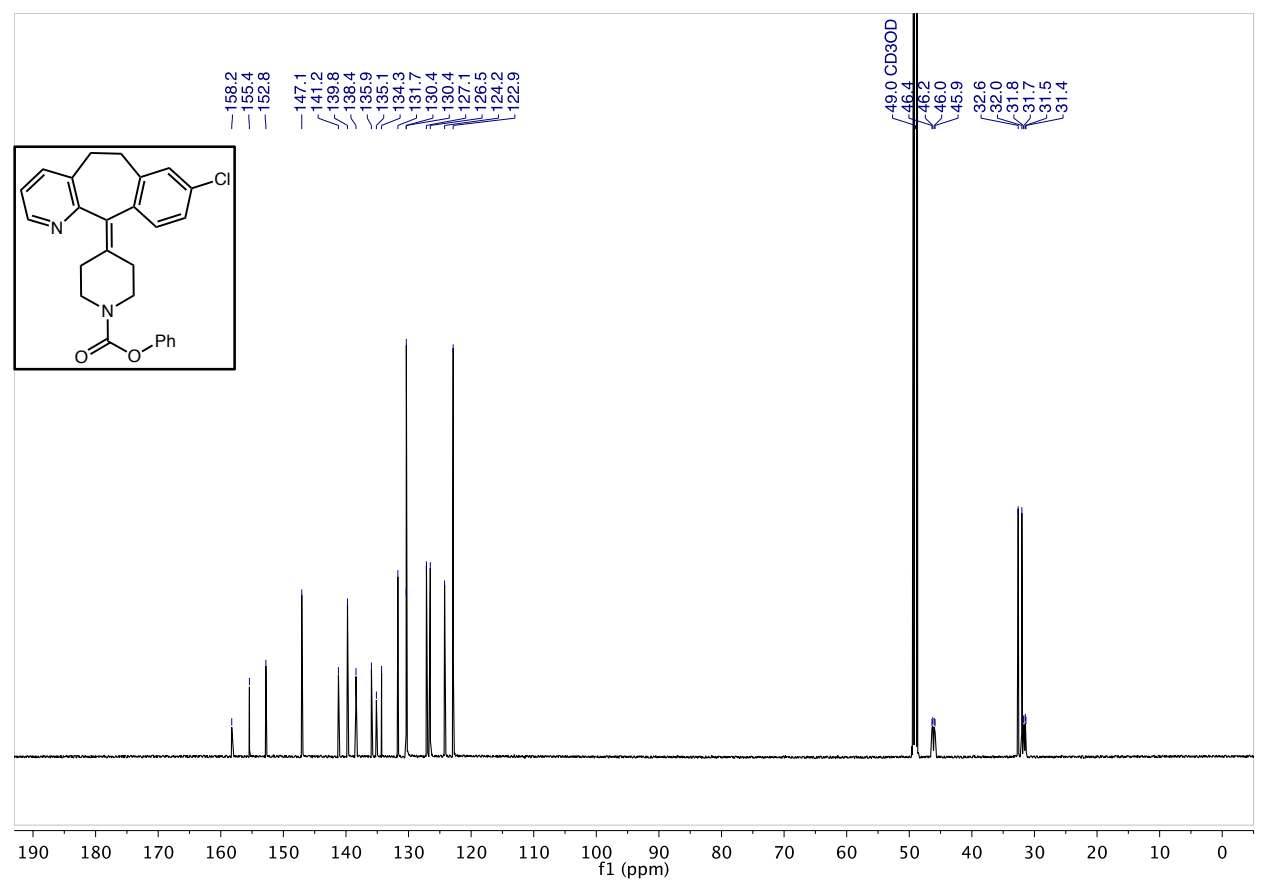


${ }^{1} \mathrm{H}$ NMR $\left(600 \mathrm{MHz}, \mathrm{CD}_{3} \mathrm{OD}\right)-\mathbf{6 k}$

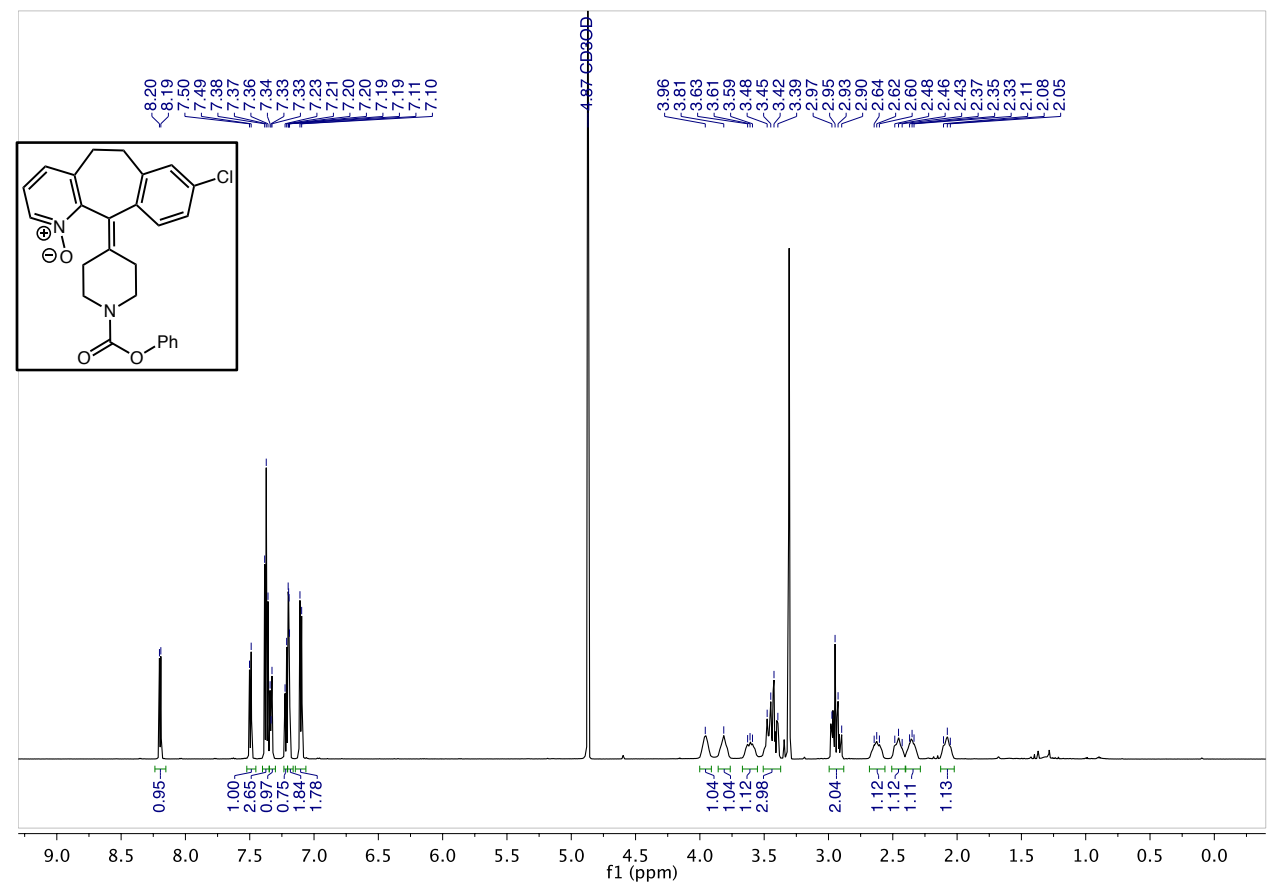

${ }^{13} \mathrm{C}$ NMR $\left(151 \mathrm{MHz}, \mathrm{CD}_{3} \mathrm{OD}\right)-\mathbf{6 k}$

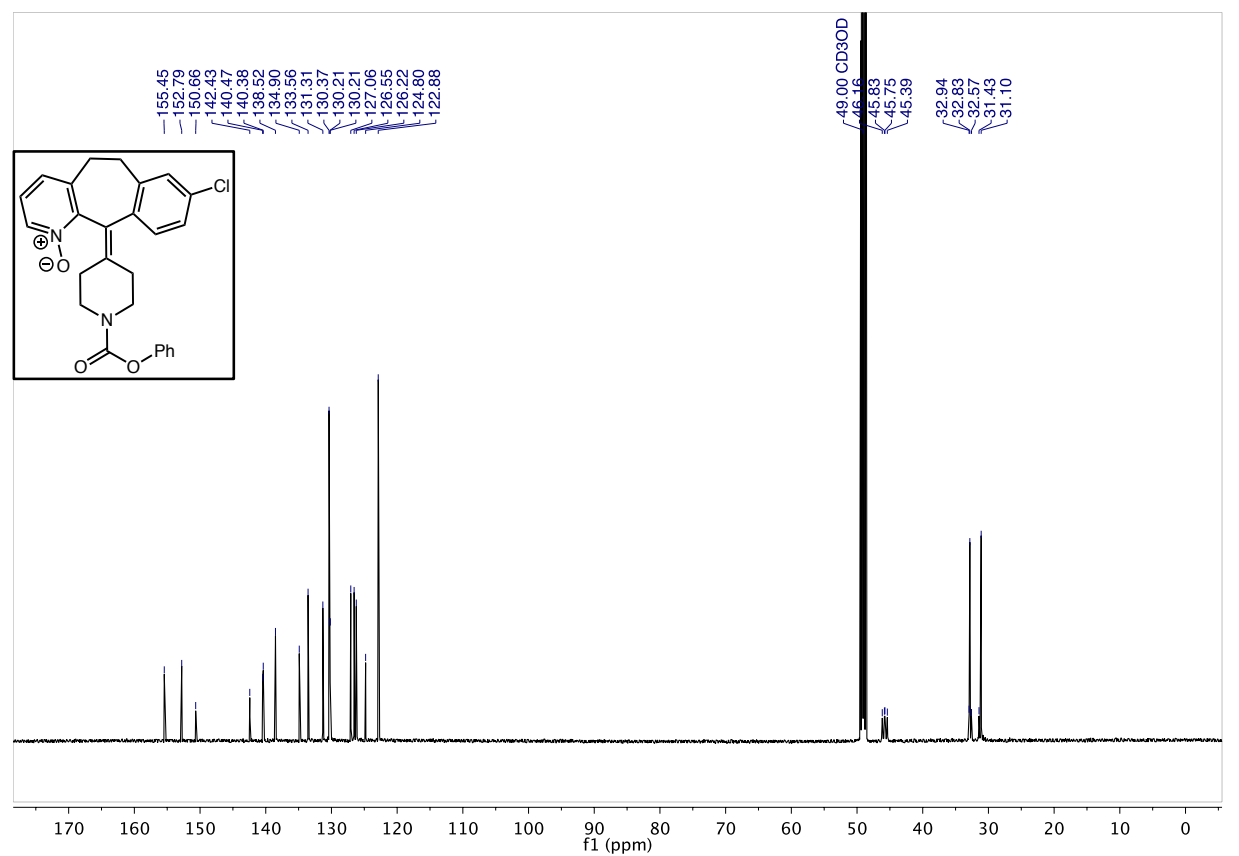


${ }^{1} \mathrm{H} \mathrm{NMR}\left(600 \mathrm{MHz}, \mathrm{CDCl}_{3}\right)-11$

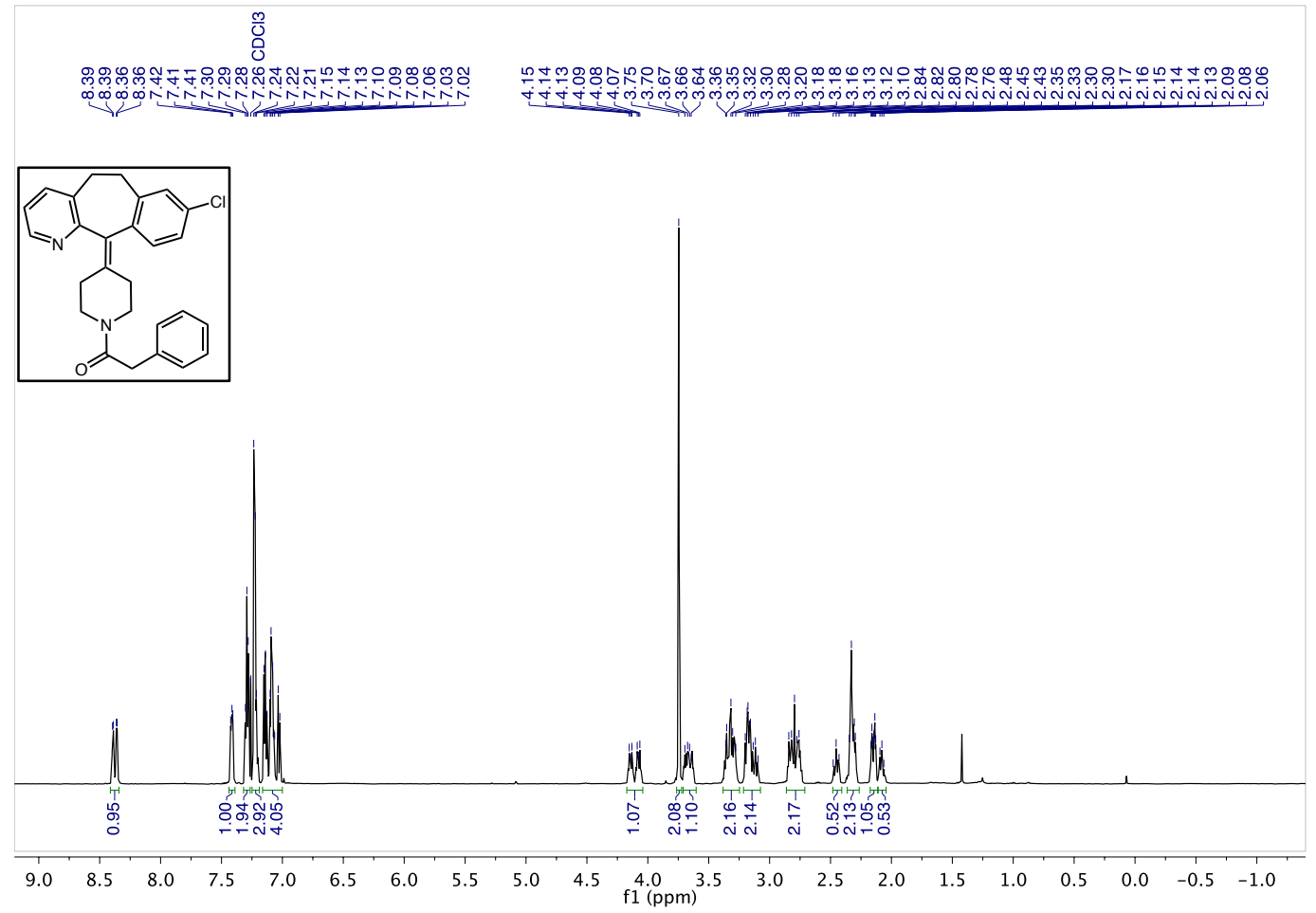

${ }^{13} \mathrm{C}$ NMR $\left(151 \mathrm{MHz}, \mathrm{CDCl}_{3}\right)-11$

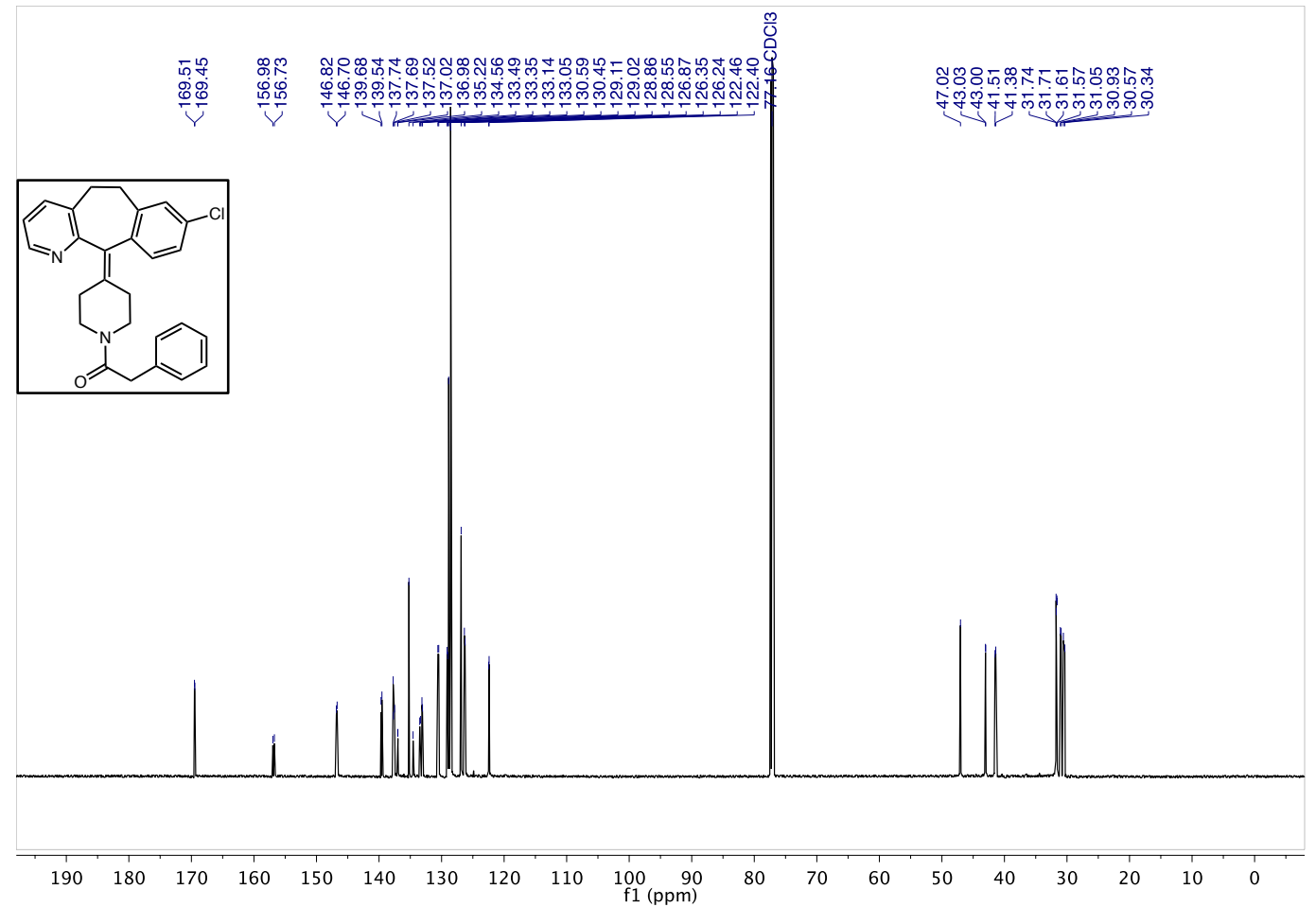


${ }^{1} \mathrm{H} \mathrm{NMR}\left(600 \mathrm{MHz}, \mathrm{CD}_{3} \mathrm{OD}\right)-61$

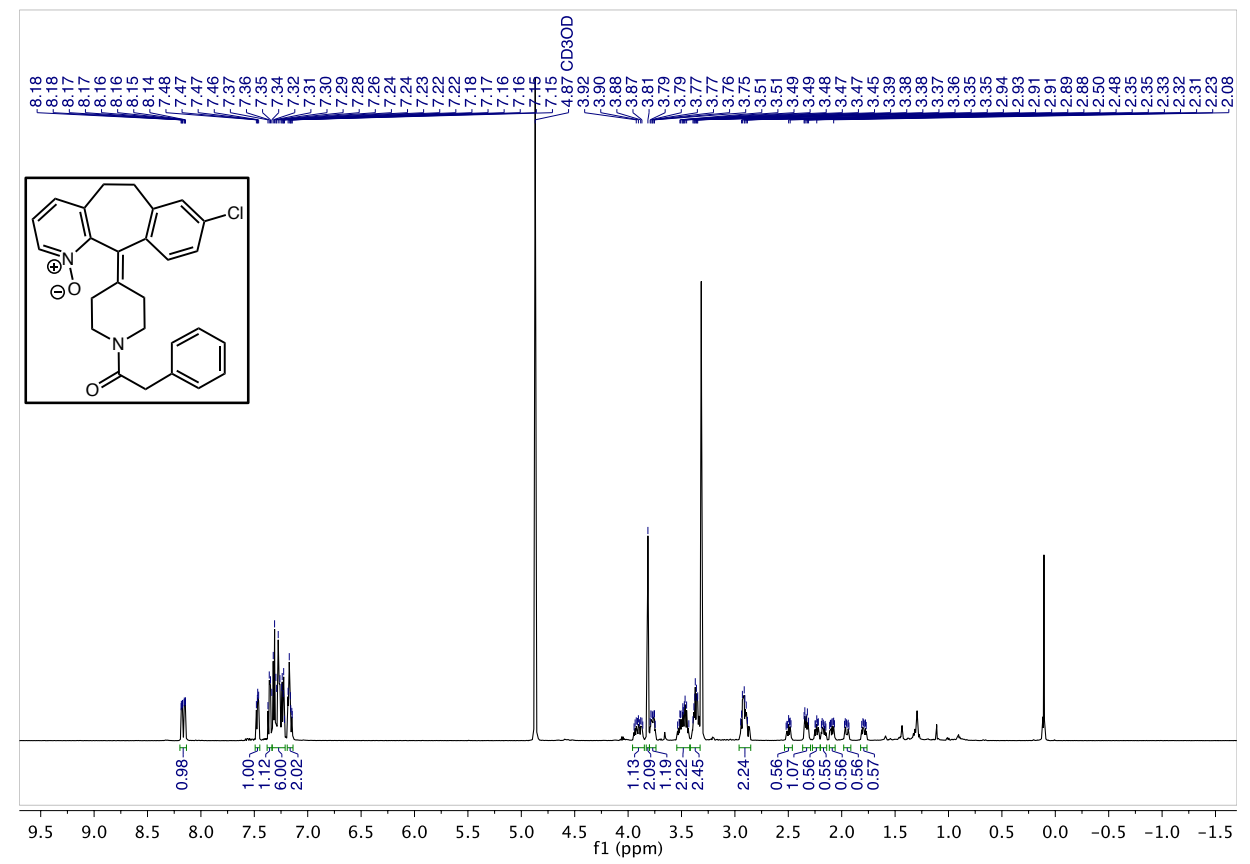

${ }^{13} \mathrm{C}$ NMR $\left(151 \mathrm{MHz}, \mathrm{CD}_{3} \mathrm{OD}\right)-61$

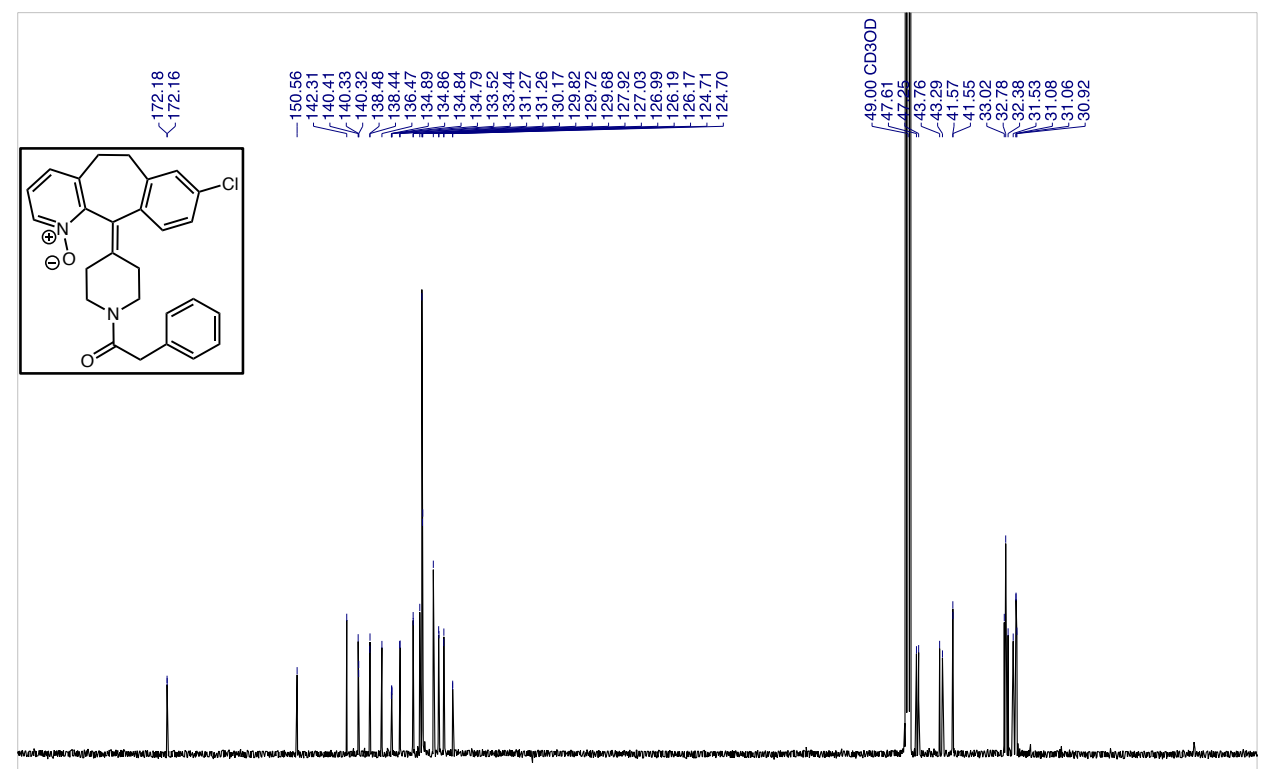

$\begin{array}{rrrrrrrrrrrrrrrrrrr}190 & 180 & 170 & 160 & 150 & 140 & 130 & 120 & 110 & \begin{array}{r}100 \\ \mathrm{f} 1(\mathrm{ppm})\end{array} & 80 & 70 & 60 & 50 & 40 & 30 & 20 & 10 & 0\end{array}$ 
${ }^{1} \mathrm{H} \mathrm{NMR}\left(600 \mathrm{MHz}, \mathrm{CDCl}_{3}\right)-\mathbf{1 m}$

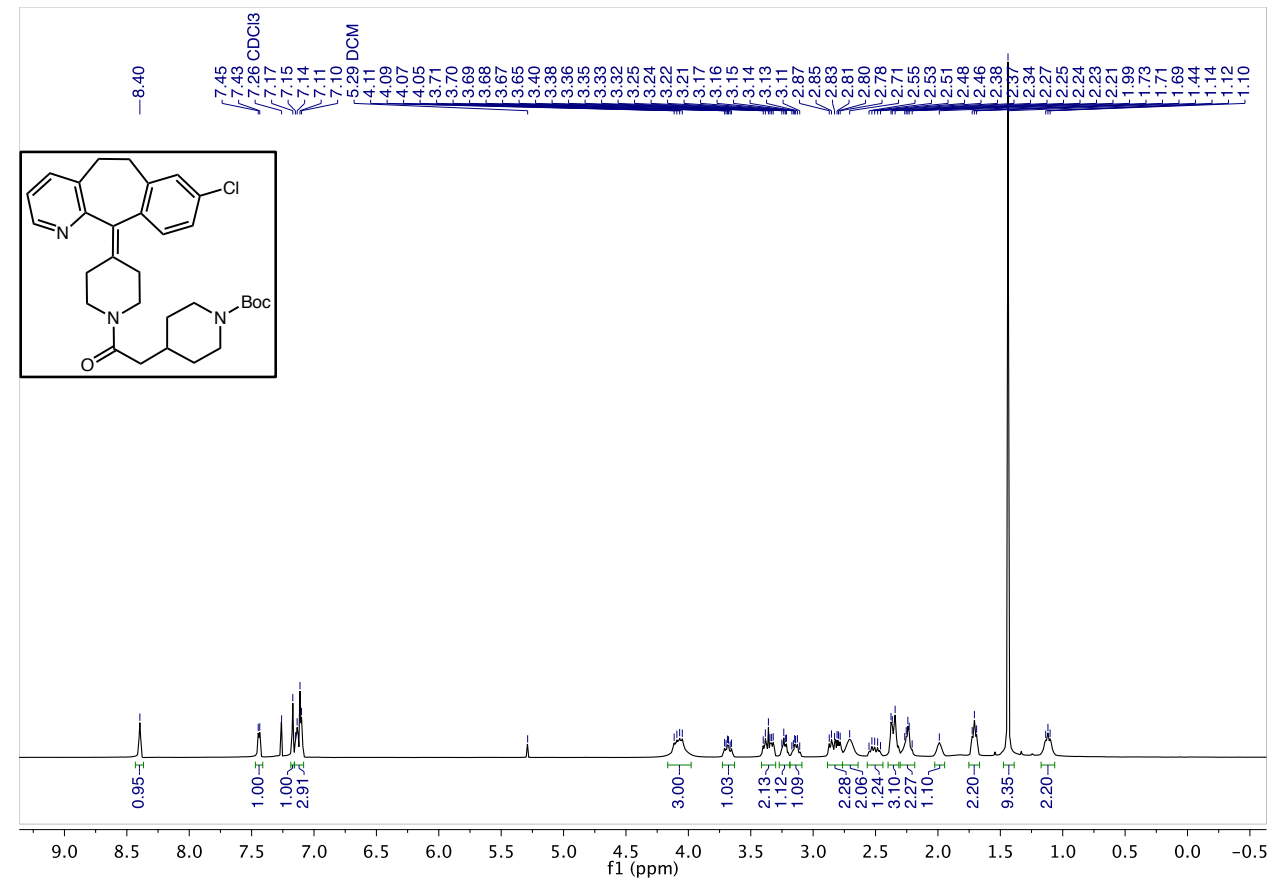

${ }^{13} \mathrm{C} \mathrm{NMR}\left(151 \mathrm{MHz}, \mathrm{CDCl}_{3}\right)-\mathbf{1 m}$
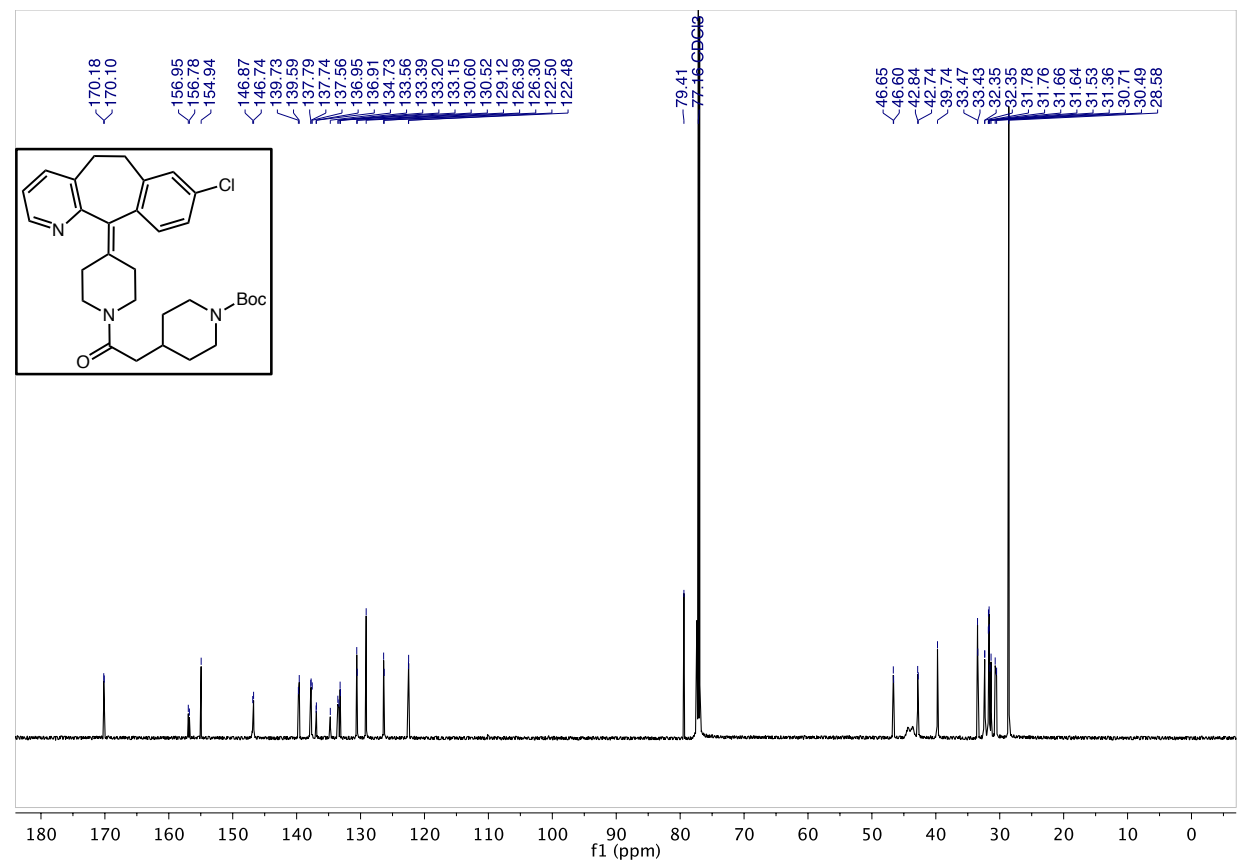
${ }^{1} \mathrm{H}$ NMR $\left(600 \mathrm{MHz}, \mathrm{CD}_{3} \mathrm{OD}\right)-6 \mathrm{~m}$

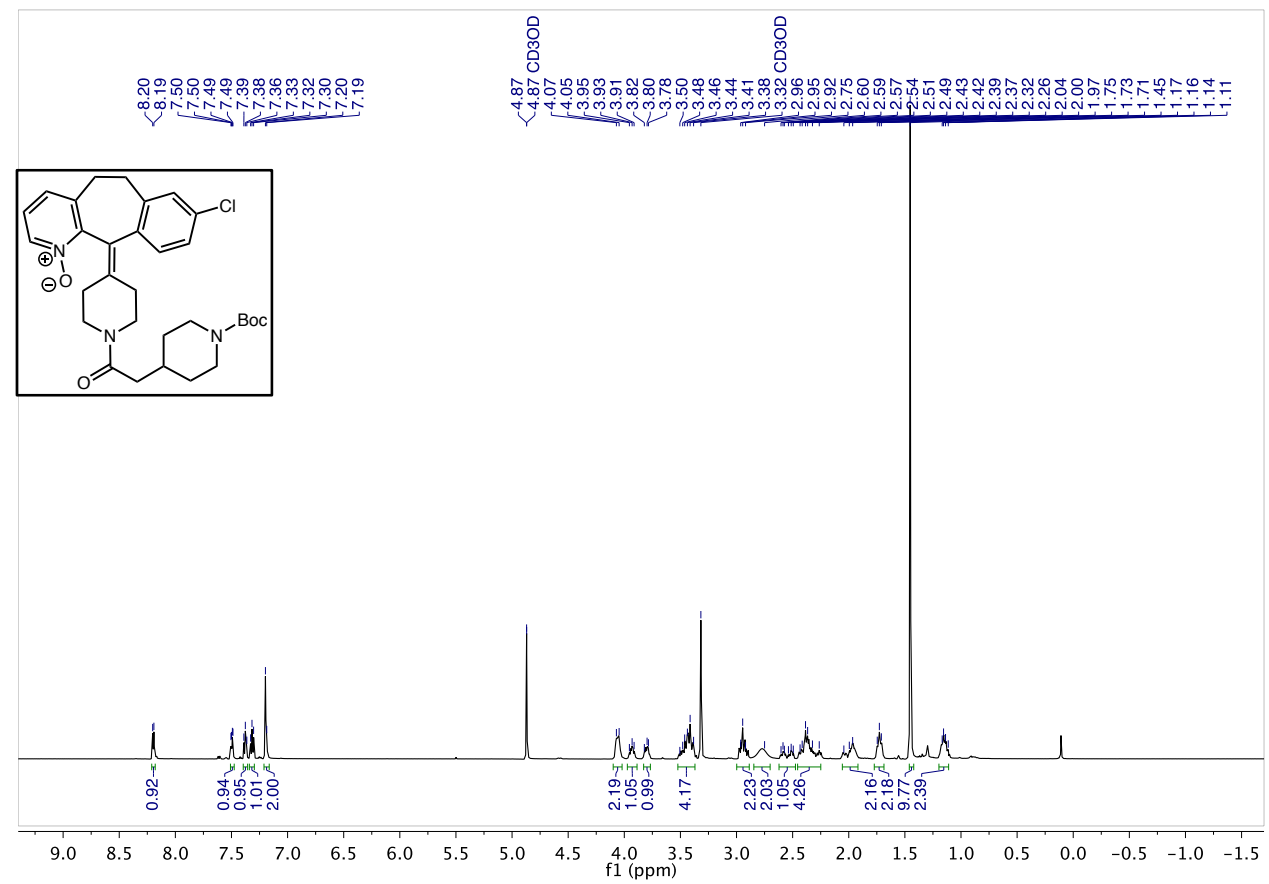

${ }^{13} \mathrm{C}$ NMR $\left(151 \mathrm{MHz}, \mathrm{CD}_{3} \mathrm{OD}\right)-6 \mathrm{~m}$

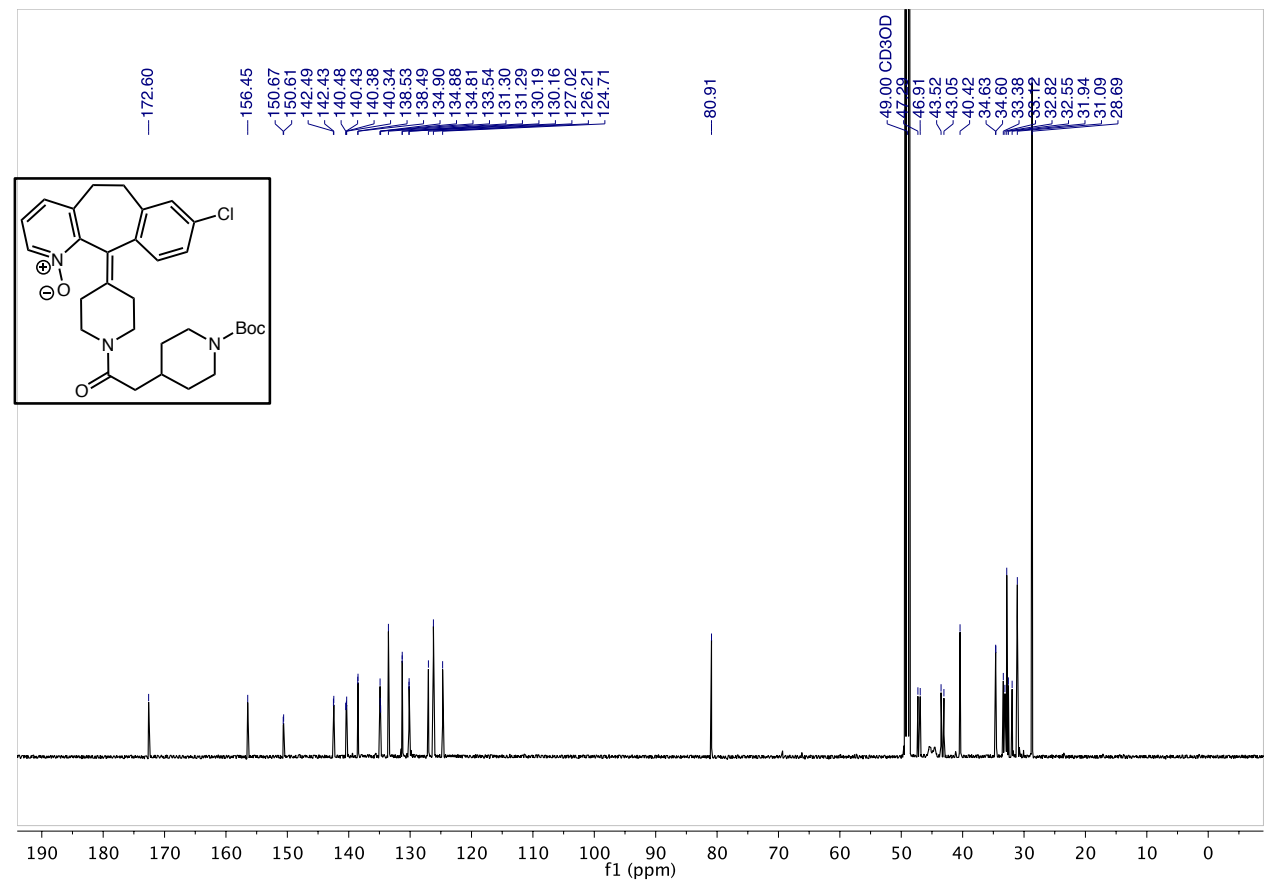




\section{Experimental Enantiomerization Barrier of $6 \mathrm{~h}$}

\section{A. General Procedure}

To an oven-dried 1-dram vial equipped with a stir bar was added a solution $(4.3 \mu \mathrm{M})$ of $6 \mathbf{h}(5.0 \mathrm{mg},>99: 1 \mathrm{er})$ in diethylene glycol dibutyl ether (diglyme, $2 \mathrm{~mL}$ ). The vial was sealed with a puncturable septa-lined cap and heated in an oil bath (equipped with a temperature probe) at $100^{\circ} \mathrm{C}$. Aliquots of $50 \mu \mathrm{L}$ were withdrawn from the reaction vessel and quickly diluted in $50 \mu \mathrm{L}$ of $\mathrm{EtOH} / \mathrm{Hex}(1: 1 \mathrm{v} / \mathrm{v})$ at $0{ }^{\circ} \mathrm{C}$ before analysis. The enantiomeric ratio was measured by Chiral HPLC (Chiralpak IB column, 10\% EtOH/hexanes, $0.7 \mathrm{~mL} / \mathrm{min}$ flowrate, $2 \mu \mathrm{L}$ injection, 25 ${ }^{\circ} \mathrm{C}, 254 \mathrm{~nm}$ ) at time points spanning $1 \mathrm{~min}$ to $3 \mathrm{hrs}$ (Table S2). As a note, some decomposition of $6 \mathbf{h}$ was observed over time, but this material did not coelute with the enantiomers. Plots of ee versus time, and of $\ln (1 / \mathrm{ee})$ versus time were generated from the data described in Tables S2-3, and resulted in the respective graphs shown below (Figure S2). Racemization rate constants $\left(k_{r a c}\right)$ were derived from the slopes of the lines shown in Figure S2. The rate constants to enantiomerization, kenant, were obtained by halving $k_{r a c}$ values. Using a rearranged form (Eq. $\mathrm{S} 1$ ) of the Eyring equation [where R - universal gas constant $=1.99 \times 10^{-3} \mathrm{kcal} /(\mathrm{mol} \mathrm{K}), \mathrm{T}=373 \mathrm{~K}, h-\mathrm{Planck}$ 's constant $\left.=6.63 \times 10^{-34}\left(\mathrm{~m}^{2} \mathrm{~kg}\right) / \mathrm{s}^{2}\right)$, and $k_{B}$ - Boltzmann constant $\left.\left.=1.38 \times 10^{-23}\left(\mathrm{~m}^{2} \mathrm{~kg}\right) / \mathrm{s}^{2} \mathrm{~K}\right)\right]$, the free energies of racemization $\left(\Delta G^{*} \mathrm{rac}\right)$ and enantiomerization $\left(\Delta G^{*}\right.$ enant $)$ at $100{ }^{\circ} \mathrm{C}$ were determined in $\mathrm{kcal} / \mathrm{mol}$. These values are summarized in Table S4.

$\Delta G_{\text {enant }}^{\ddagger}=R T *\left[\ln \left(\frac{k_{\text {enant }} h}{k_{B} T}\right)\right]$

Eq. S1

Table S2. Enantiomeric excess measured over time at $100^{\circ} \mathrm{C}$, Trial 1

\begin{tabular}{ccccc} 
Entry & Time (s) & \% major enantiomer & ee & In (1/ee) \\
\hline A & 63.0 & 98.7 & 0.97 & 0.026 \\
B & 165.0 & 97.7 & 0.95 & 0.047 \\
C & 281.0 & 96.1 & 0.92 & 0.081 \\
D & 421.0 & 94.2 & 0.88 & 0.123 \\
E & 565.0 & 92.5 & 0.85 & 0.163 \\
F & 687.0 & 91.0 & 0.82 & 0.198 \\
G & 819.0 & 89.5 & 0.79 & 0.236 \\
H & 973.0 & 87.8 & 0.76 & 0.280 \\
I & 1155.0 & 85.8 & 0.72 & 0.334 \\
J & 1642.0 & 80.9 & 0.62 & 0.481 \\
K & 2183.0 & 76.2 & 0.52 & 0.646 \\
L & 2983.0 & 70.0 & 0.40 & 0.916 \\
M & 5521.0 & 59.1 & 0.18 & 1.704 \\
N & 12008.0 & 51.2 & 0.02 & 3.730
\end{tabular}


Table S3. Enantiomeric excess measured over time at $100^{\circ} \mathrm{C}$, Trial 2

\begin{tabular}{ccccc} 
Entry & Time (s) & \% major enantiomer & ee & In (1/ee) \\
\hline A & 62.0 & 99.4 & 0.99 & 0.012 \\
B & 263.0 & 97.4 & 0.95 & 0.053 \\
C & 590.0 & 93.4 & 0.87 & 0.142 \\
D & 886.0 & 89.9 & 0.80 & 0.226 \\
E & 1168.0 & 86.8 & 0.74 & 0.307 \\
F & 1716.0 & 81.1 & 0.62 & 0.475 \\
G & 2620.0 & 73.4 & 0.47 & 0.759 \\
H & 4408.0 & 62.8 & 0.26 & 1.363
\end{tabular}
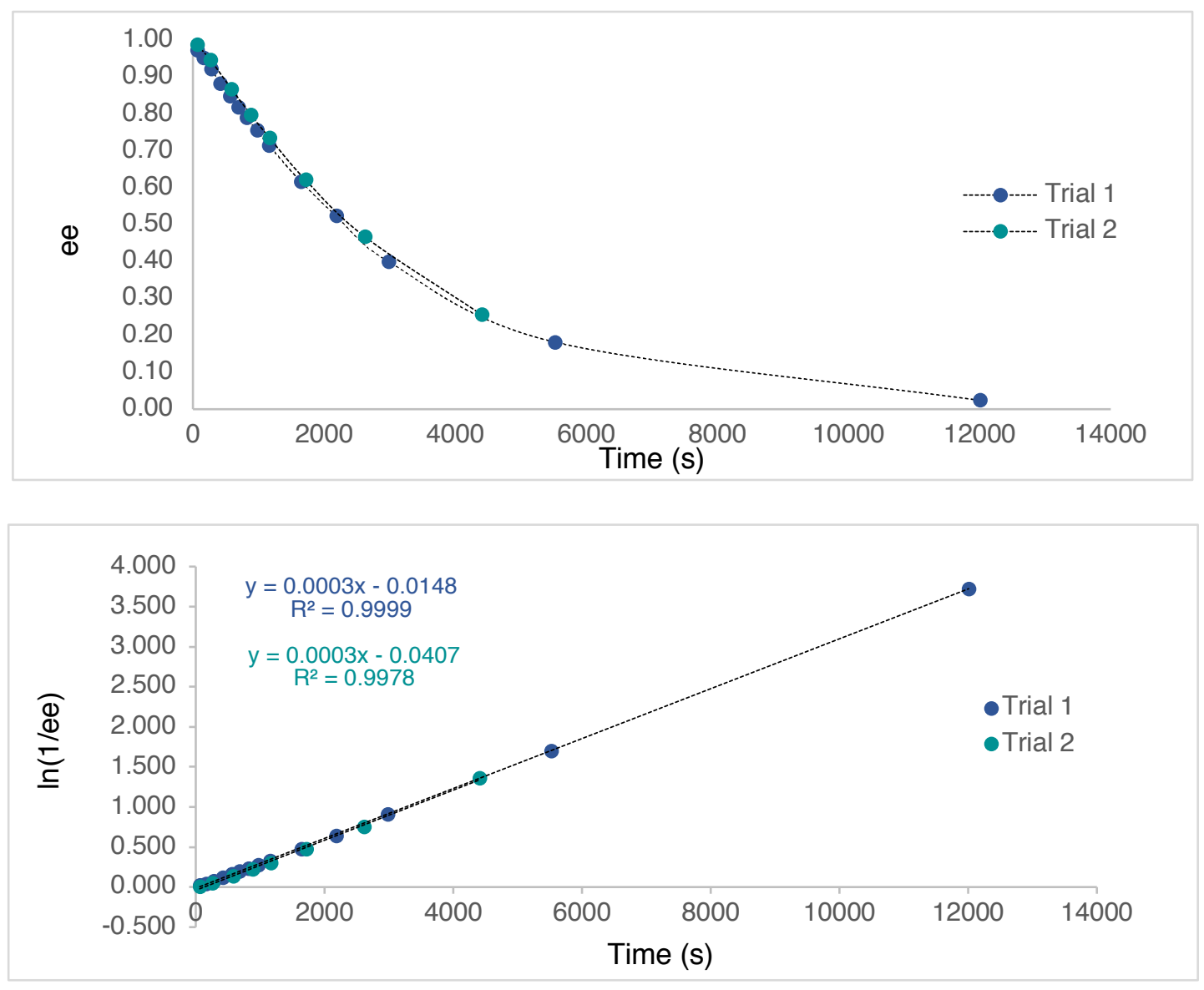

Figure S2. Plots measuring enantiomeric excess vs. time at $100^{\circ} \mathrm{C}$ (two trials).

Table S4. Experimentally determined barriers of racemization and enantiomerization of $6 \mathrm{~h}$ at $100^{\circ} \mathrm{C}$.

\begin{tabular}{ccccc} 
Trial & $\boldsymbol{k}_{\text {rac }}\left(\mathbf{s}^{-1}\right)$ & $\Delta G \neq_{\text {rac }}(\mathrm{kcal} / \mathrm{mol})$ & $\boldsymbol{k}_{\text {enant }}$ & $\Delta G \neq_{\text {enant }}(\mathrm{kcal} / \mathrm{mol})$ \\
\hline 1 & $3.11 \times 10^{-4}$ & 28.02 & $1.56 \times 10^{-4}$ & 28.54 \\
2 & $3.13 \times 10^{-4}$ & 28.02 & $1.56 \times 10^{-4}$ & 28.54
\end{tabular}




\section{B. Enantiomeric Stability at $37^{\circ} \mathrm{C}$}

The general procedure for determining the barrier to enantiomerization (S58) was followed, except $6 \mathrm{~h}(92: 8 \mathrm{er})$ was heated at $37^{\circ} \mathrm{C}$ for $15 \mathrm{~h}$. Chiral HPLC (Chiralpak IB column, $10 \% \mathrm{EtOH} /$ hexanes, $0.7 \mathrm{~mL} / \mathrm{min}$ flowrate, 5 $\mu \mathrm{L}$ injection, $\left.25^{\circ} \mathrm{C}, 254 \mathrm{~nm}\right)$ traces before $(\mathrm{t}=0)$ and after $(\mathrm{t}=15 \mathrm{~h})$ heating are shown below.

$\mathbf{t}=\mathbf{0}$

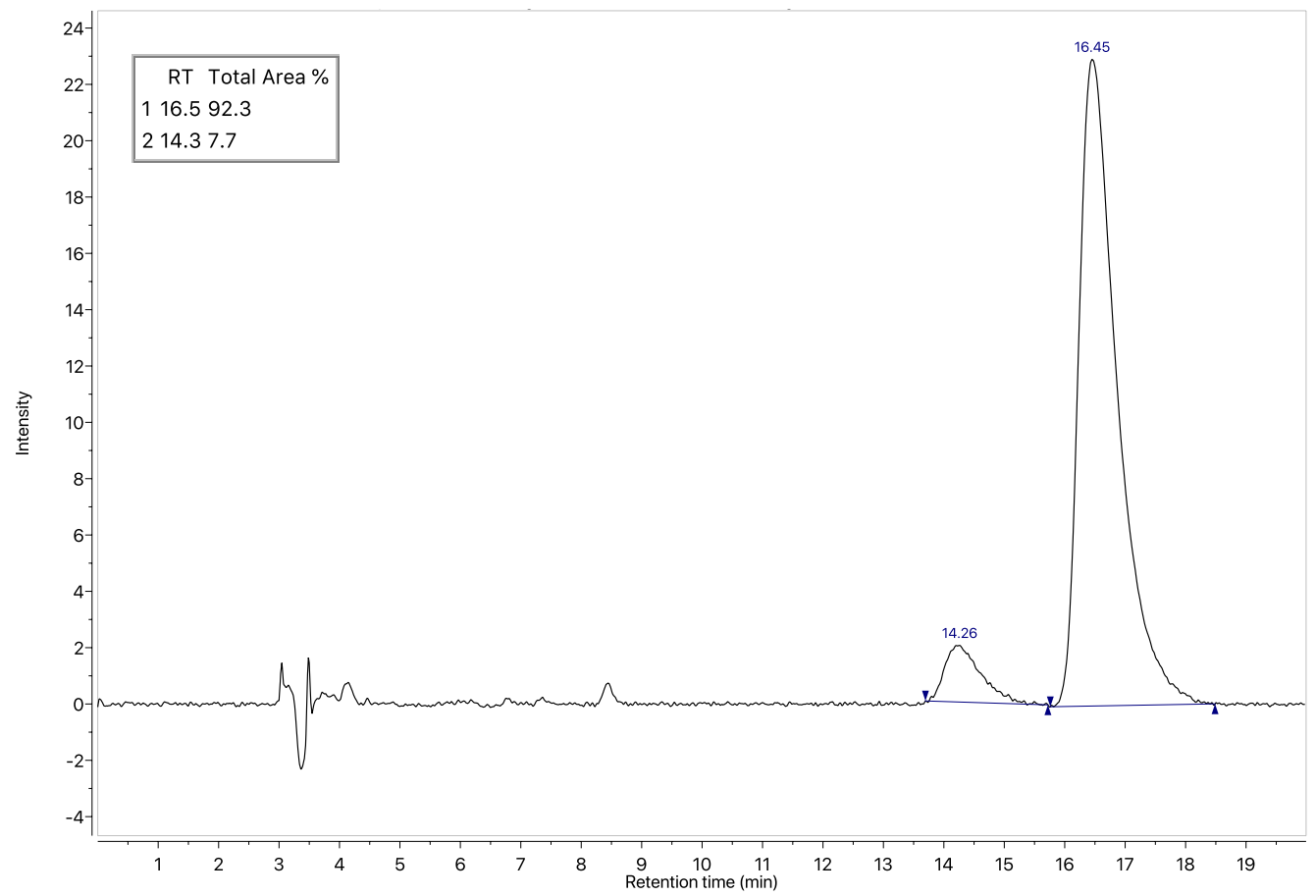

$\mathrm{t}=15 \mathrm{~h}$

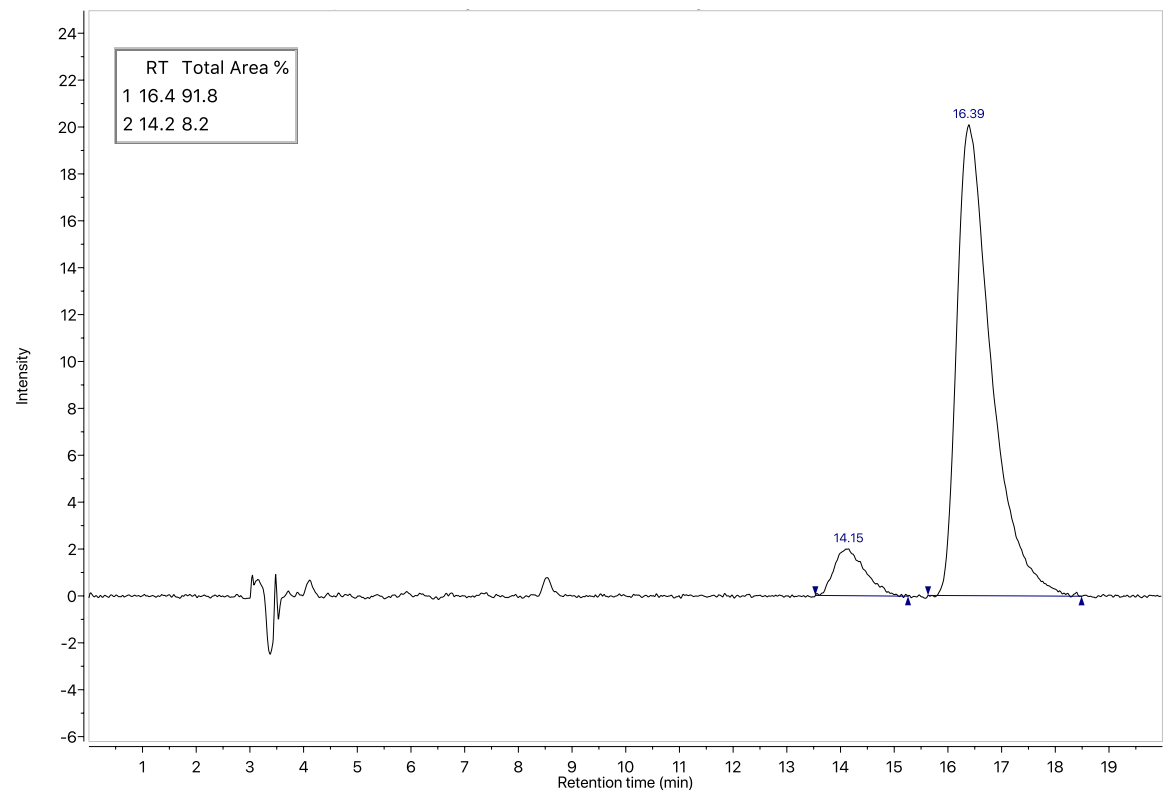




\section{Chiral Resolution of Loratadine $N$-Oxides (6a)}

Racemic loratadine $\mathrm{N}$-oxide 6 a was synthesized according to standard racemic standard preparation using loratadine $1 \mathrm{a}(0.100 \mathrm{~g}, 0.26 \mathrm{mmol}, 1.0$ equiv $)$. The crude material was first purified using normal-phase column chromatography (SNAP Ultra 50g, gradient $=0-8 \% \mathrm{MeOH} / \mathrm{DCM}$ over $8 \mathrm{CV}$, then $8 \% \mathrm{MeOH} / \mathrm{DCM}$ over $2 \mathrm{CV}$, then 8 $10 \% \mathrm{MeOH} / \mathrm{DCM}$ over $3 \mathrm{CV}$ ). This purified material was dissolved in $0.6 \mathrm{~mL}$ of ethanol, and $0.2 \mathrm{~mL}$ were loaded directly onto a Chiralflash IC column $(100 \mathrm{~mm}$, i.d. $30 \mathrm{~mm}$, particle size $20 \mu \mathrm{m})$ fitted with adaptors for use with a MPLC Biotage Isolera Prime ( $80 \% \mathrm{EtOH} / \mathrm{Hex}, 12 \mathrm{~mL} / \mathrm{min}$; Figure S3, top). Fractions containing each enantiomer were collected and pooled individually to afford $7.6 \mathrm{mg}(+)-6 \mathrm{a}$ (peak 2). A second chiral column (Figure S3, bottom) was performed under the same conditions on the first enantiomer to elute to afford $5.6 \mathrm{mg}$ of (-)-6a (peak 1). The enantiomeric purity of each samples was confirmed using HPLC (Chiralpak IB column, $80 \% \mathrm{EtOH} / \mathrm{hex}-$ anes, $0.7 \mathrm{~mL} / \mathrm{min}$ flowrate, $5 \mu \mathrm{L}$ injection, $\left.25^{\circ} \mathrm{C}, 254 \mathrm{~nm}\right):(-)-6 \mathrm{a}\left(t_{\mathrm{R}}=6.8 \mathrm{~min}\right), 98: 2 \mathrm{er} ;(+)-6 \mathrm{a}\left(t_{\mathrm{R}}=7.7 \mathrm{~min}\right)$, $>99: 1$ er.

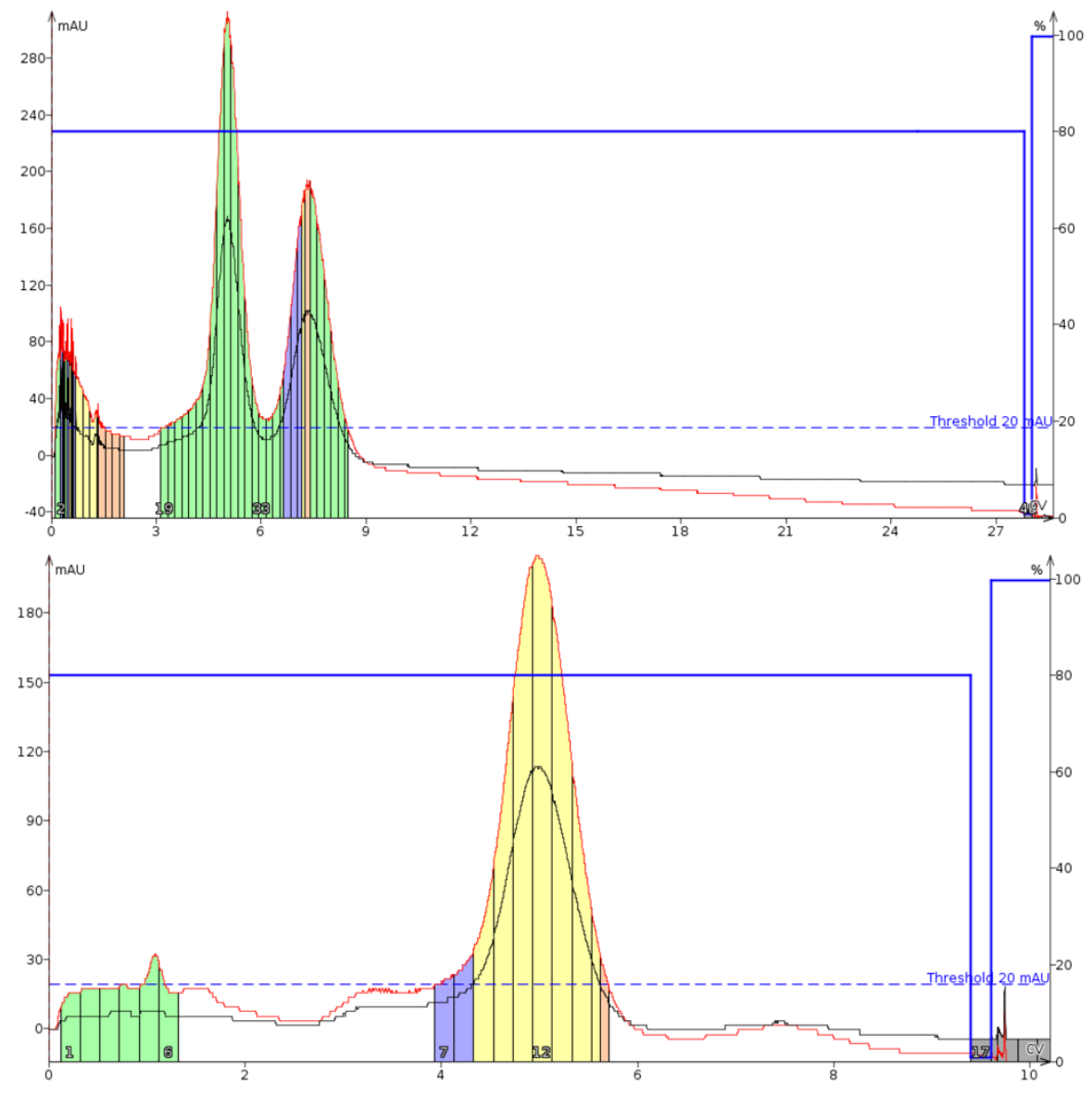

Figure S3. Preparative chiral chromatography of 6a: column 1 (top) and column 2 (bottom). 


\section{Racemic 6a}

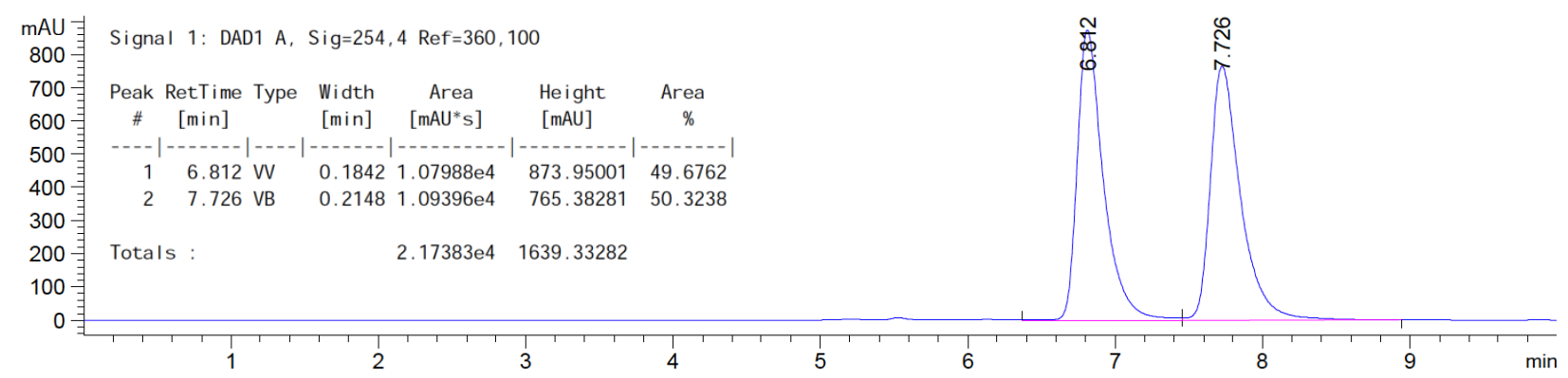

(-)-6a

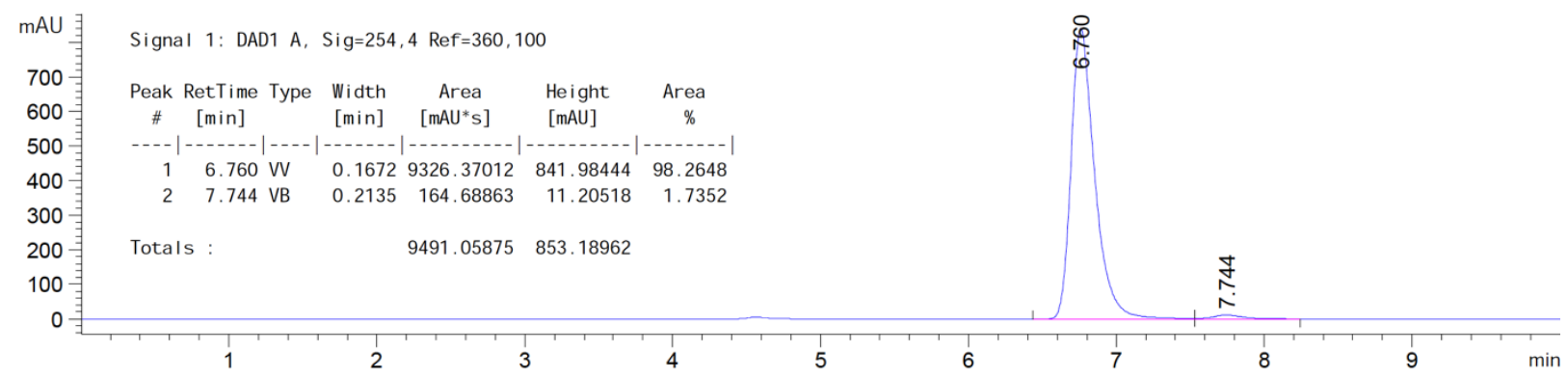

(+)-6a

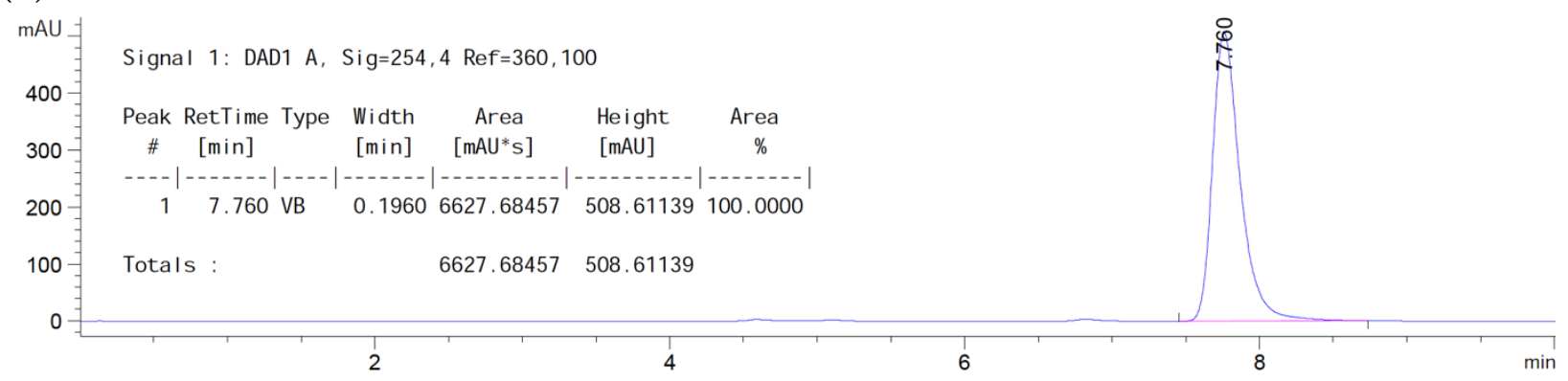




\section{VIIII. Antihistamine Activity Testing of Loratadine Analogs}

\section{Fluorescence assay to measure intracellular calcium:}

Free intracellular calcium was measured in 384-well plates on a FLIPR Tetra plate reader (Molecular Devices). HeLa cells from sub-confluent flasks were harvested with trypsin, resuspended in Hank's Balanced Salt solution (HBSS) and counted. Cells were plated in poly-D-lysine coated 384-well plates (\#781946, Greiner Bio-One) at 15,000 cells/well in $25 \mu \mathrm{L}$. Cells were allowed to attached for $20 \mathrm{~min}$ at $37^{\circ} \mathrm{C}$ prior to calcium indicator dye loading and test compound incubation. Compounds were dispensed (20-100 nL) from DMSO stocks with an Echo 550 acoustic dispenser (LabCyte) into an intermediate plate (Matrix 4312, Thermo Scientific) and $100 \mu \mathrm{L}$ of dye loading solution (PBX Calcium Assay kit \#640175, BD Biosciences) was added. The concentration of compound in the dye solution was $2 \mathrm{x}$ final and the final DMSO in the assay plate was $0.01-0.05 \%$. Cells were loaded with $25 \mu \mathrm{L}$ dye containing test compounds for $30 \mathrm{~min}$ at $37^{\circ} \mathrm{C}$ and then transferred to room temperature for $30 \mathrm{~min}$. Histamine stock $(100 \mathrm{mM})$ was made fresh each day in distilled water, diluted to the appropriate concentration ( $3 \mathrm{x}$ final) in HBSS and the working solution added to a Matrix 4312 plate. After a $20 \mathrm{sec}$ baseline read on the FLIPR instrument, histamine was added $(25 \mu \mathrm{L})$ and fluorescence measured (Ex 470-495 nm, Em 515-575 nm) every 2 sec for 100 $\mathrm{sec}$, followed by every $5 \mathrm{sec}$ for $250 \mathrm{sec}$. The peak fold change in fluorescence during the first $100 \mathrm{sec}$ was measured with ScreenWorks software (Molecular Devices). To measure the inhibitory effect of compounds on the histamine response, ${ }^{3}$ the peak fold increase in fluorescence for each well was normalized to the average fold change observed in wells treated with DMSO only and challenged with $12.5 \mu \mathrm{M}$ histamine. The normalized mean peak fluorescence was then plotted versus compound concentration and a two parameter, variable slope logistic function (Max fixed to 1.0 and Min to zero) was fit to the data using Prism 7 (GraphPad Software). On each assay plate, there were three replicate wells for each compound concentration and the reported $\mathrm{IC}_{50}$ values are the mean of three separate experiments.

A

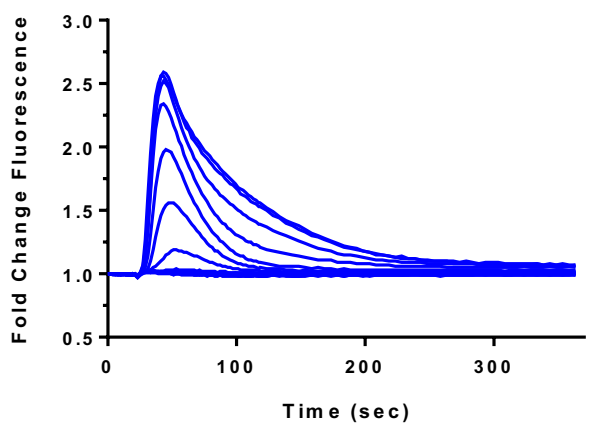

B

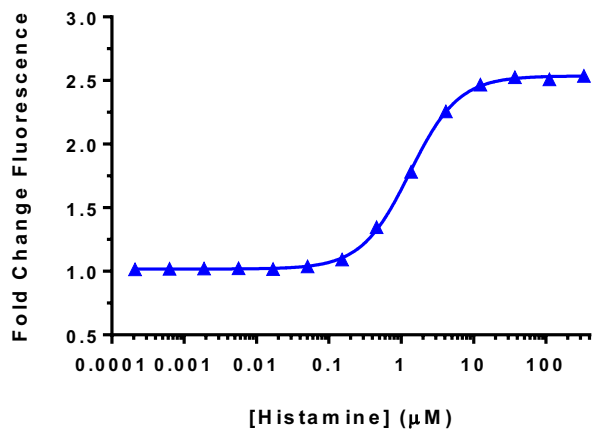

Figure S4. Histamine increases intracellular calcium in HeLa cells. (a) Representative traces of peak fluorescence intensity changes in response to increasing concentrations of histamine $(0-333 \mu \mathrm{M})$ expressed as fold change over basal levels. Histamine was added at $\mathrm{t}=20 \mathrm{sec}$. (b) Plot of the fold change in peak fluorescence intensity vs. histamine concentration. The symbols are the average of 14 replicates. The solid line is a four-parameter logistic fit to the data with the values: $\mathrm{EC}_{50}=1.31 \mu \mathrm{M}$, slope $=1.29, \mathrm{Max}=2.54, \mathrm{Min}=1.02$. 
A

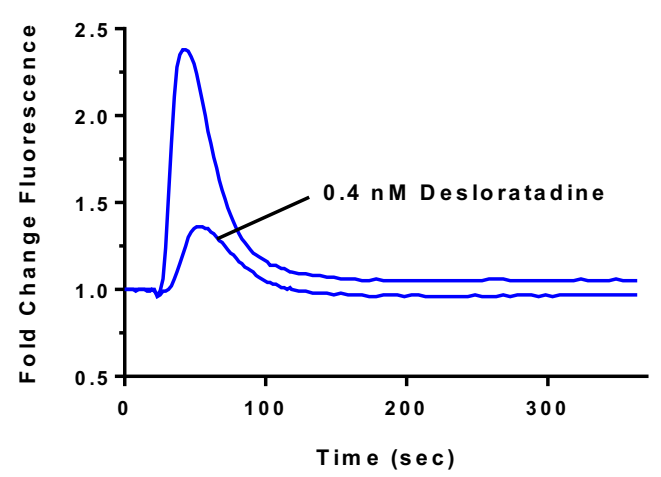

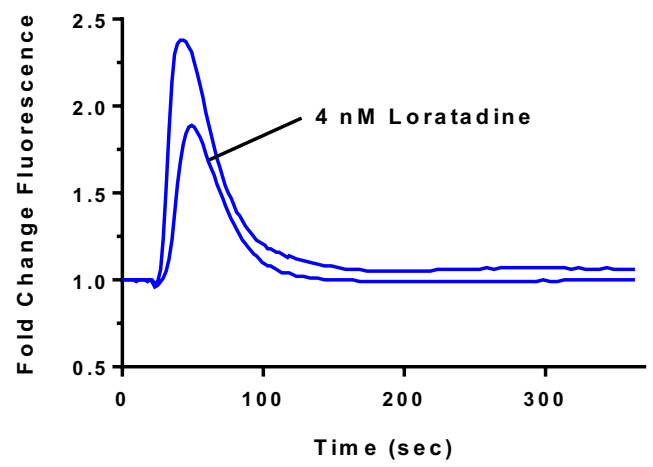

Figure S5. Desloratadine and loratadine inhibit the histamine-induced calcium elevation in HeLa cells. (a) Representative traces of the peak fold change in fluorescence intensity in response to application of $12.5 \mu \mathrm{M}$ histamine for cells preincubated with vehicle (DMSO) or $0.4 \mathrm{nM}$ desloratadine. (b) Representative traces of the peak fold change in fluorescence intensity in response to application of $12.5 \mu \mathrm{M}$ histamine for cells preincubated with vehicle (DMSO) or $4 \mathrm{nM}$ loratadine.

Table S5. Antihistamine activity of loratadine analogs. Average $\mathrm{IC}_{50}$ values are reported in Figure 4.

\begin{tabular}{l|l|l|l}
\hline \multirow{2}{*}{ Compound } & \multicolumn{3}{|c}{ IC $_{50}(\mathrm{nM})$} \\
\cline { 2 - 4 } & Trial 1 & Trial 2 & Trial 3 \\
\hline Desloratadine (2) & $<0.1$ & $<0.1$ & $<0.1$ \\
Loratadine (1a) & 5.9 & 3.8 & 6.5 \\
1h & 7.0 & 4.2 & 5.8 \\
$(-)-6 a(98: 2$ er) & 14000 & 12200 & 14700 \\
$(+)-6 a(>99: 1$ er) & $>25000$ & $>25000$ & $>25000$ \\
$(-)-6 h(98: 2$ er) & 266 & 194 & 264 \\
$(+)-6 h(>99: 1$ er) & 13200 & 11200 & 13200
\end{tabular}




\section{DFT Computational Studies}

All DFT calculations were performed using the Gaussian 16, Revision A.03. The B3LYP ${ }^{5-7}$ hybrid functionals have been used in combination with Pople's $6-311++\mathrm{G}(2 \mathrm{~d}, 3 \mathrm{p})$ basis sets. Gibbs free energies refer to gas phase conditions at standard temperature $(298.15 \mathrm{~K})$ and pressure $(1 \mathrm{~atm})$ unless otherwise indicated. The crystal structure of $6 e^{1}$ was used as a starting point for ground state optimization. No imaginary frequencies were observed for ground states. Transitions states were verified by occurrence of one imaginary frequency (shown below). Their assignment to stationary points was verified by visual inspection and optimization of distorted transition state structures in both directions of the imaginary frequency. All data was obtained on triple- $\zeta$ geometry optimized B3LYP/6$311++\mathrm{G}(2 \mathrm{~d}, 3 \mathrm{p})$ level including the D3 version of Grimme's dispersion correction. ${ }^{9}$ In the following section, the abbreviations GS (ground state) and TS (transition state) are used.

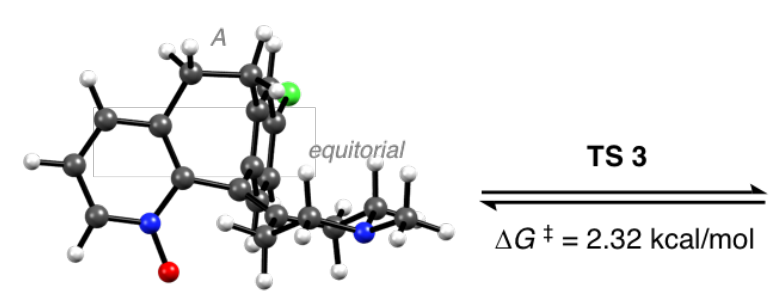

TS $1 \| \Delta G^{\ddagger}=8.77 \mathrm{kcal} / \mathrm{mol}$

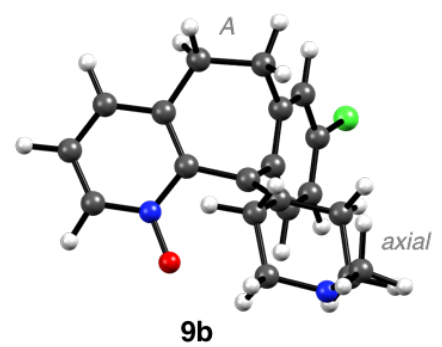

$9 \mathbf{b}$

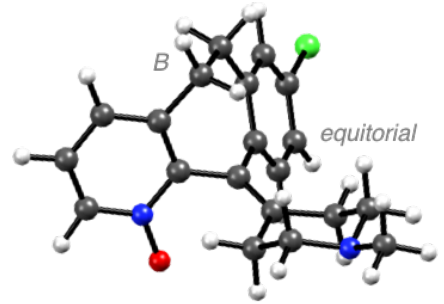

9d
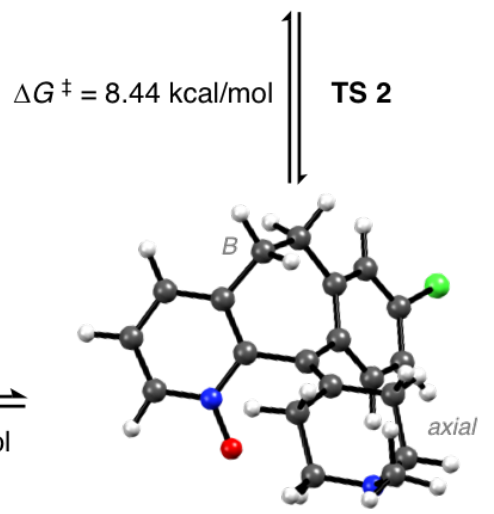

$9 c$

\begin{tabular}{ccccccc} 
Entry & GS/TS & Conformer & $\mathbf{E}$ (Hartree) & $\mathbf{G}_{298 \mathbf{~ K}}$ (Hartree) & $\boldsymbol{\Delta} \mathbf{G} \neq_{298 \mathbf{~ K}}(\mathbf{k c a l} / \mathbf{m o l})$ & $\boldsymbol{\eta}_{\text {imag }}\left(\mathbf{c m}^{-1}\right)$ \\
\hline 1 & GS & 9a (A, equitorial) & -1419.615863 & -1419.664781 & 0.40 & None \\
2 & GS & 9b (A, axial) & -1419.612122 & -1419.660506 & 3.08 & None \\
3 & GS & 9c (B, axial) & -1419.612861 & -1419.661115 & 2.70 & None \\
4 & GS & 9d (B, equitorial) & -1419.616492 & -1419.66542 & 0.00 & None \\
5 & TS & 1 & -1419.596713 & -1419.646515 & 8.77 & -243.97 \\
6 & TS & 2 & -1419.598048 & -1419.64765 & 8.44 & -246.32 \\
7 & TS & 3 & -1419.612155 & -1419.660692 & 2.32 & -116.55 \\
8 & TS & 4 & -1419.608619 & -1419.656429 & 2.31 & -118.21
\end{tabular}


9a GS, (A, equitorial)

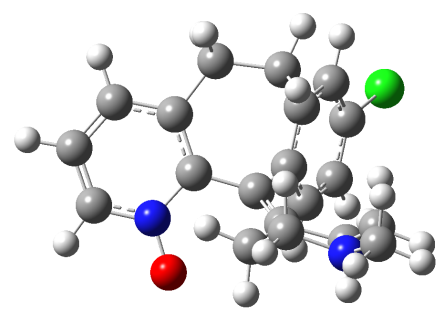

$\mathrm{Cl} \quad 5.40401100 \quad-1.40756800 \quad-0.16793200$

$\begin{array}{lllll}\mathrm{O} & -1.60811500 & 1.80111500 & -2.20019400\end{array}$

$\mathrm{N} \quad \begin{array}{llll}-1.21567800 & 2.36866900 & -1.11939000\end{array}$

$\begin{array}{lllll}\text { C } & -0.60117000 & 1.63937800 & -0.10570800\end{array}$

$\begin{array}{lllll}\text { C } & -0.40031300 & 0.19098900 & -0.36370200\end{array}$

C $\quad \begin{array}{llll}\text { C } & 1.03192000 & -0.22258100 & -0.34876700\end{array}$

$\begin{array}{lllll}\text { C } & 1.72088700 & -0.54773700 & -1.51454300\end{array}$

$\mathrm{H} \quad \begin{array}{llll}\mathrm{C} & 1.20197500 & -0.51041800 & -2.46248100\end{array}$

C $\quad 3.06255200 \quad-0.91343600 \quad-1.47097100$

$\mathrm{H} \quad 3.59818900 \quad-1.16777100 \quad-2.37331500$

C $\quad 3.70761200 \quad-0.94422400 \quad-0.24353400$

$\begin{array}{llll}\text { C } & 3.04212400 & -0.61678200 & 0.93114400\end{array}$

$\mathrm{H} \quad 3.56836000 \quad-0.65333300 \quad 1.87460400$

$\begin{array}{llll}\text { C } & 1.69967400 & -0.25258600 & 0.88337300\end{array}$

$\begin{array}{llll}\text { C } & 0.92760000 & 0.13004700 & 2.11832800\end{array}$

$\mathrm{H} \quad 1.49620500 \quad-0.12738200 \quad 3.01242800$

$\mathrm{H} \quad-0.00039800 \quad-0.44297500 \quad 2.15385400$

C $\quad \begin{array}{llll}0.60802600 & 1.63256100 & 2.18339400\end{array}$

$\mathrm{H} \quad 0.03551600 \quad 1.82118300 \quad 3.09515500$

$\mathrm{H} \quad \begin{array}{llll}\mathrm{H} & 1.54998500 & 2.17381700 & 2.30486000\end{array}$

$\begin{array}{llll}\text { C } & -0.13253200 & 2.30423100 & 1.03204700\end{array}$

$\begin{array}{lllll}\text { C } & & -0.33277600 & 3.67995600 & 1.15458800\end{array}$

$\begin{array}{lllll}\mathrm{H} & 0.01955000 & 4.19477100 & 2.03793500\end{array}$

$\begin{array}{lllll}\mathrm{C} & -0.98050900 & 4.37755300 & 0.14787600\end{array}$

$\mathrm{H} \quad-1.15115100 \quad 5.44190300 \quad 0.22033000$

$\begin{array}{lllll}\text { C } & -1.40918700 & 3.71060600 & -0.97463000\end{array}$

$\mathrm{H} \quad-1.90358100 \quad 4.16880800 \quad-1.81463100$

C $\quad-1.41068700 \quad-0.66624200 \quad-0.56226500$

$\begin{array}{llll}\text { C } & -1.21021600 & -2.13778600 & -0.81114600\end{array}$

$\mathrm{H} \quad-1.52365700 \quad-2.36076600 \quad-1.83547400$

$\mathrm{H} \quad-0.16585300 \quad-2.42595700-0.71515600$

$\begin{array}{lllll}\text { C } & -2.06425100 & -2.98007800 & 0.14358900\end{array}$

$\mathrm{H} \quad-1.97134500 \quad-4.03511600 \quad-0.11774300$

$\mathrm{H} \quad-1.67710100 \quad-2.86518200 \quad 1.17343200$

N $\quad-3.47137700 \quad-2.60395500 \quad 0.06756000$

$\begin{array}{llll}\text { C } & -3.65388600 & -1.19838800 & 0.41465800\end{array}$

$\mathrm{H} \quad-3.34690500 \quad-1.00801800 \quad 1.46045900$

$\mathrm{H} \quad-4.71590000 \quad-0.95930800 \quad 0.34437600$

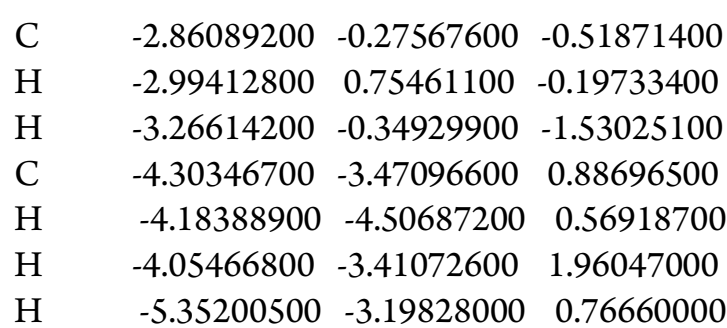

9b GS, (A, axial)

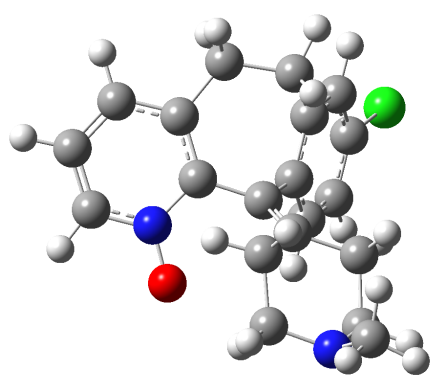

$\begin{array}{llll}\mathrm{Cl} & 5.33022500 & -1.57713100 & -0.51964600 \\ \mathrm{O} & -2.00175600 & 1.18087600 & -1.85348400 \\ \mathrm{~N} & 1.39860100 & 2.02532700 & -1.09677500 \\ \mathrm{C} & -0.57820800 & 1.60031100 & -0.05789500 \\ \mathrm{C} & 0.99621600 & -0.32082500 & -0.07690800 \\ \mathrm{C} & 1.41837000 & -1.00182000 & -1.21611900 \\ \mathrm{H} & 0.70180300 & -1.22170800 & -1.99553100 \\ \mathrm{C} & 2.74553100 & -1.39341800 & -1.36215600 \\ \mathrm{H} & 3.07420000 & -1.92356000 & -2.24349500 \\ \mathrm{C} & 3.64753600 & -1.08769700 & -0.35397500 \\ \mathrm{C} & 3.25101300 & -0.40158100 & 0.78713700 \\ \mathrm{H} & 3.97452400 & -0.17966000 & 1.55894400 \\ \mathrm{C} & 1.92205000 & -0.01375100 & 0.93003400 \\ \mathrm{C} & 1.43234100 & 0.74785600 & 2.13354500 \\ \mathrm{H} & 2.19096300 & 0.74250000 & 2.91683200 \\ \mathrm{H} & 0.55081900 & 0.24896500 & 2.53911200 \\ \mathrm{C} & 1.09500300 & 2.21511100 & 1.81982300 \\ \mathrm{H} & 0.72325400 & 2.68377500 & 2.73462600 \\ \mathrm{H} & 2.02747300 & 2.73155900 & 1.57752300 \\ \mathrm{C} & 0.11322000 & 2.54977600 & 0.70197600 \\ \mathrm{C} & 0.07463300 & 3.90772400 & 0.44017600 \\ \mathrm{H} & 0.45109900 & 4.64393100 & 1.03276200 \\ \mathrm{C} & -0.92943100 & 4.30568900 & -0.57481500 \\ \mathrm{H} & -1.09472200 & 5.35034800 & -0.79502400 \\ \mathrm{C} & -1.57526200 & 3.35627600 & -1.33010700 \\ \mathrm{H} & -2.23992900 & 3.56702400 & -2.15095500 \\ \mathrm{C} & -0.41263200 & 0.13319900 & 0.10203000 \\ \mathrm{C} & -1.41582000 & -0.69339200 & 0.43246300\end{array}$ 


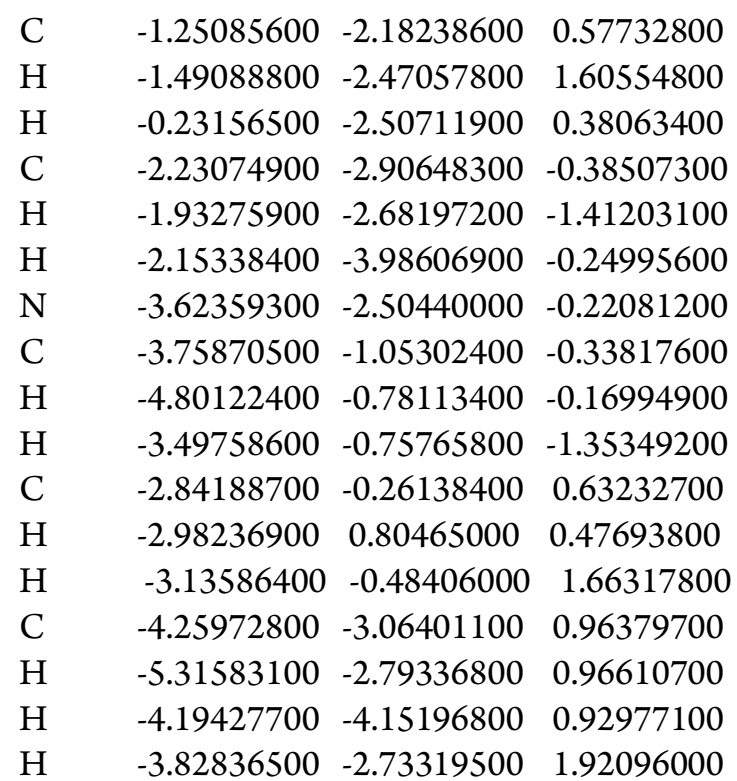

9c GS, (B, axial)

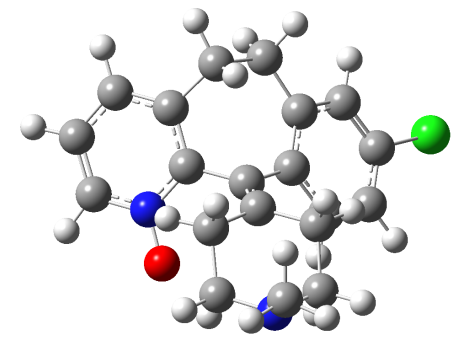

$\begin{array}{llll}\mathrm{Cl} & 5.29246300 & -1.66837700 & -0.53499700 \\ \mathrm{C} & 1.00577100 & -0.26356300 & 0.07022400 \\ \mathrm{C} & 1.31837700 & -1.27763200 & -0.84721700 \\ \mathrm{H} & 0.51591400 & -1.70691400 & -1.42936800 \\ \mathrm{C} & 2.61437700 & -1.72350200 & -1.04605300 \\ \mathrm{H} & 2.83248600 & -2.50496400 & -1.75829200 \\ \mathrm{C} & 3.63275000 & -1.12760300 & -0.31610700 \\ \mathrm{C} & 3.36206700 & -0.10360500 & 0.57308600 \\ \mathrm{H} & 4.17720000 & 0.35222800 & 1.11840100 \\ \mathrm{C} & 2.05499600 & 0.35116200 & 0.77721200 \\ \mathrm{O} & -1.71284500 & 1.07765400 & -2.08468400 \\ \mathrm{~N} & -1.26865900 & 1.95712800 & -1.26184400 \\ \mathrm{C} & -0.64982000 & 1.58971200 & -0.08936700 \\ \mathrm{C} & -0.18321200 & 2.55865900 & 0.79709400 \\ \mathrm{C} & -0.34181000 & 3.90750900 & 0.50348000 \\ \mathrm{H} & 0.01162600 & 4.66405500 & 1.18983400 \\ \mathrm{C} & -0.96913300 & 4.26105200 & -0.68762700 \\ \mathrm{H} & -1.11493300 & 5.29685100 & -0.95833300\end{array}$

$\begin{array}{llll}\mathrm{C} & -1.42013000 & 3.28678500 & -1.54650300 \\ \mathrm{H} & -1.91392600 & 3.47406700 & -2.48532400 \\ \mathrm{C} & -0.42276700 & 0.15179200 & 0.19005500 \\ \mathrm{C} & 1.91036300 & 1.50312000 & 1.76399800 \\ \mathrm{H} & 2.54166300 & 2.32073400 & 1.40498500 \\ \mathrm{H} & 2.34324800 & 1.18898900 & 2.71698500 \\ \mathrm{C} & 0.50859900 & 2.06920100 & 2.04231100 \\ \mathrm{H} & 0.61037700 & 2.89199800 & 2.75047900 \\ \mathrm{H} & -0.10687600 & 1.30659500 & 2.52136800 \\ \mathrm{C} & -1.43042600 & -0.67135200 & 0.52091800 \\ \mathrm{C} & -1.27559000 & -2.12901100 & 0.86098800 \\ \mathrm{H} & -1.66504500 & -2.29646100 & 1.86989100 \\ \mathrm{H} & -0.23800100 & -2.45346100 & 0.85913400 \\ \mathrm{C} & -2.10415500 & -2.98310300 & -0.13659200 \\ \mathrm{H} & -1.66637900 & -2.87588700 & -1.13202800 \\ \mathrm{H} & -2.04191200 & -4.03753700 & 0.13509000 \\ \mathrm{~N} & -3.50851900 & -2.59482000 & -0.21825400 \\ \mathrm{C} & -3.63700000 & -1.16702200 & -0.50529500 \\ \mathrm{H} & -4.69506400 & -0.90381200 & -0.51835900 \\ \mathrm{H} & -3.23374100 & -0.96941700 & -1.49866100 \\ \mathrm{C} & -2.87644600 & -0.25706600 & 0.49766000 \\ \mathrm{H} & -3.00972600 & 0.78262300 & 0.21041200 \\ \mathrm{H} & -3.30755900 & -0.38132900 & 1.49585200 \\ \mathrm{C} & -4.30714100 & -3.04395800 & 0.91416100 \\ \mathrm{H} & -5.35348900 & -2.79437300 & 0.73717400 \\ \mathrm{H} & -4.23310400 & -4.12833300 & 1.00138600 \\ \mathrm{H} & -4.01963600 & -2.61005600 & 1.88391100\end{array}$

\section{9d GS, (B, equitorial)}

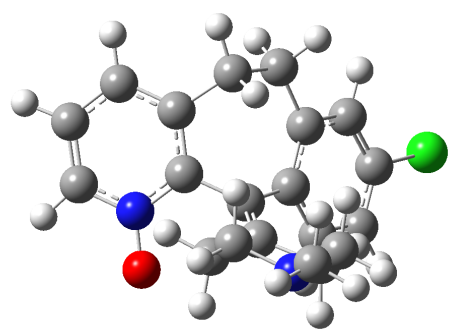

$\begin{array}{llll}\mathrm{Cl} & 5.45132800 & -1.44778000 & -0.15423000 \\ \mathrm{C} & 1.06315800 & -0.24696700 & -0.20435600 \\ \mathrm{C} & 1.64277600 & -0.93243500 & -1.28187200 \\ \mathrm{H} & 1.02454100 & -1.15546400 & -2.13960100 \\ \mathrm{C} & 2.97510400 & -1.30991000 & -1.28781400 \\ \mathrm{H} & 3.40225100 & -1.83602700 & -2.12821100 \\ \mathrm{C} & 3.75539500 & -0.98155100 & -0.18838700 \\ \mathrm{C} & 3.22035500 & -0.28198800 & 0.87785200 \\ \mathrm{H} & 3.85491400 & -0.02949600 & 1.71638900 \\ \mathrm{C} & 1.87561100 & 0.10338600 & 0.88867900\end{array}$ 
C $\quad-1.37199700 \quad-0.74337100 \quad-0.53341400$

$\begin{array}{lllll}\text { C } & -1.19988200 & -2.23942400 & -0.56974500\end{array}$

$\mathrm{H} \quad-1.39203000 \quad-2.59433700 \quad-1.58667000$

$\mathrm{H} \quad-0.18990400 \quad-2.53870000-0.30045500$

$\begin{array}{llll}\text { C } & -2.19727000 & -2.92482100 & 0.37323400\end{array}$

$\mathrm{H} \quad-2.11408900 \quad-4.00709900 \quad 0.26474400$

$\mathrm{H} \quad-1.93046800 \quad-2.68141300 \quad 1.41867400$

$\mathrm{N} \quad \begin{array}{llll}-3.57064200 & -2.53245800 & 0.07826200\end{array}$

C $\quad-3.74082000 \quad-1.08993000 \quad 0.21718000$

$\mathrm{H} \quad-3.55459300 \quad-0.76875400 \quad 1.25934300$

$\mathrm{H} \quad-4.77662300 \quad-0.83885700 \quad-0.01532300$

C $\quad-2.80404700 \quad-0.32239700 \quad-0.72180200$

$\mathrm{H} \quad-2.93779200 \quad 0.74605000 \quad-0.57006200$

$\mathrm{H} \quad-3.08646700 \quad-0.53562300 \quad-1.75583600$

$\begin{array}{lllll}\mathrm{O} & -1.15944800 & 1.74532900 & -2.42681600\end{array}$

$\mathrm{N} \quad \begin{array}{llll}-0.98786700 & 2.31055400 & -1.28947500\end{array}$

$\begin{array}{lllll}\text { C } & -0.63061700 & 1.58303500 & -0.17744800\end{array}$

$\begin{array}{llll}\text { C } & -0.45517100 & 2.21115500 & 1.05385000\end{array}$

$\begin{array}{llll}\text { C } & -0.64442300 & 3.58288800 & 1.17024600\end{array}$

$\mathrm{H} \quad \begin{array}{llll}\mathrm{H} & -0.51751300 & 4.07570400 & 2.12381300\end{array}$

$\begin{array}{lllll}\text { C } & -1.00613200 & 4.30400000 & 0.03594500\end{array}$

$\mathrm{H} \quad-1.16464900 \quad 5.37197900 \quad 0.07807700$

C $\quad-1.17120200 \quad 3.66173800 \quad-1.16834600$

$\mathrm{H} \quad-1.44838600 \quad 4.14404600 \quad-2.09075200$

$\begin{array}{lllll}\text { C } & -0.37695100 & 0.12905400 & -0.31668200\end{array}$

$\begin{array}{llll}\text { C } & 1.42979500 & 0.88919200 & 2.11555300\end{array}$

$\begin{array}{llll}\mathrm{H} & 2.06619500 & 1.77586700 & 2.18453800\end{array}$

$\mathrm{H} \quad \begin{array}{llll}1.65645500 & 0.29016100 & 3.00099100\end{array}$

$\begin{array}{lllll}\text { C } & -0.03526300 & 1.34388000 & 2.21152200\end{array}$

$\mathrm{H} \quad-0.15822700 \quad 1.89566700 \quad 3.14380500$

$\mathrm{H} \quad-0.68806800 \quad 0.47143900 \quad 2.26565000$

$\begin{array}{llll}\text { C } & -4.52526700 & -3.26633600 & 0.89428400\end{array}$

$\mathrm{H} \quad-4.40832500 \quad-4.33727000 \quad 0.72775300$

$\mathrm{H} \quad-4.40226000 \quad-3.07157300 \quad 1.97350200$

$\mathrm{H} \quad-5.54130300 \quad-2.98853300 \quad 0.61368900$

\section{TS, 1}

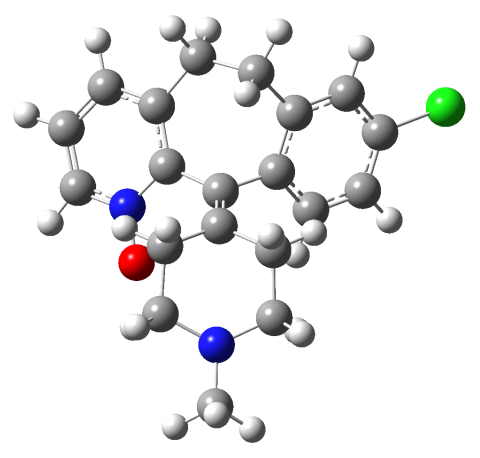

C $\quad \begin{array}{llll}-1.77713600 & 3.18050700 & -1.43287100\end{array}$

$\begin{array}{lllll}\text { C } & -1.06358400 & 4.22427600 & -0.89382500\end{array}$

$\begin{array}{lllll}\text { C } & -0.08950900 & 3.96812600 & 0.05671800\end{array}$

$\begin{array}{llll}\text { C } & 0.15262000 & 2.65764100 & 0.47282900\end{array}$

$\begin{array}{llll}\text { C } & 1.26467200 & 2.48318400 & 1.50149300\end{array}$

$\begin{array}{llll}\text { C } & 2.01576200 & 0.14483300 & 0.86434500\end{array}$

$\begin{array}{llll}\text { C } & 3.32454300 & -0.27502200 & 0.64624800\end{array}$

C $\quad 3.60430400-1.13010800 \quad-0.41249700$

C $\quad 2.60420800 \quad-1.57667300 \quad-1.26328400$

C $\quad 1.29736900-1.15285800 \quad-1.04288100$

$\begin{array}{llll}\text { C } & 0.99327300 & -0.29953800 & 0.01506100\end{array}$

$\begin{array}{llll}\text { C } & -0.39570200 & 0.17967200 & 0.26608200\end{array}$

$\begin{array}{lllll}\mathrm{C} & & -0.60434700 & 1.61262000 & -0.06703200\end{array}$

$\mathrm{Cl} \quad 5.26328800 \quad-1.65683900 \quad-0.67429100$

$\begin{array}{llll}\text { C } & -2.75249900 & -0.18815200 & 1.09534100\end{array}$

$\begin{array}{llll}\text { C } & -1.34709200 & -0.60620600 & 0.78585700\end{array}$

$\begin{array}{llll}\text { C } & -2.04346800 & -2.96136000 & 0.31852100\end{array}$

$\mathrm{H} \quad-2.53759000 \quad 3.27739300 \quad-2.18935700$

$\mathrm{H} \quad-1.27187600 \quad 5.22941400 \quad-1.23067300$

$\mathrm{H} \quad 0.48876000 \quad 4.77810400 \quad 0.47982100$

$\begin{array}{llll}\mathrm{H} & 1.00129200 & 3.08729500 & 2.37344000\end{array}$

$\mathrm{H} \quad 2.15755000 \quad 2.94792200 \quad 1.07503200$

$\mathrm{H} \quad 4.12249500 \quad 0.05132000 \quad 1.29829000$

$\mathrm{H} \quad 2.84221600 \quad-2.23969300 \quad-2.08154900$

$\mathrm{H} \quad 0.50365300 \quad-1.48334500 \quad-1.69869900$

$\mathrm{H} \quad-2.93923900 \quad 0.86027300 \quad 0.88214900$

$\mathrm{H} \quad-2.90079900 \quad-0.33402400 \quad 2.17017800$

$\mathrm{H} \quad-1.49519300 \quad-3.22666500 \quad-0.59703600$

$\mathrm{H} \quad-2.19124600 \quad-3.90127100 \quad 0.86968800$

$\begin{array}{llll}\text { C } & 1.64784300 & 1.07672400 & 1.98853700\end{array}$

$\begin{array}{llll}\mathrm{H} & 0.81902600 & 0.64706000 & 2.55377000\end{array}$

$\begin{array}{llll}\mathrm{H} & 2.48466200 & 1.18126400 & 2.67978400\end{array}$

C $\quad-4.33651500 \quad-3.28947200 \quad-0.46977900$

$\mathrm{H} \quad \begin{array}{rrrr}\mathrm{C} & -5.07333100 & -2.77281400 & -1.08872900\end{array}$

$\mathrm{H} \quad-3.90155700 \quad-4.08375000 \quad-1.08067800$

$\mathrm{H} \quad-4.87818100 \quad-3.76847500 \quad 0.36497500$

$\mathrm{N} \quad-3.31671600 \quad-2.37322600 \quad-0.03783800$

C $\quad-1.14163200 \quad-2.04483200 \quad 1.16189900$

$\mathrm{H} \quad-1.41203700 \quad-2.15221800 \quad 2.21675400$

$\mathrm{H} \quad-0.10830600 \quad-2.36512200 \quad 1.05593300$

$\begin{array}{llll}\text { C } & -3.77917400 & -1.04249500 & 0.32232700\end{array}$

$\mathrm{H} \quad-4.69460900 \quad-1.10936400 \quad 0.92855500$

$\mathrm{H} \quad-4.03598600 \quad-0.50047400 \quad-0.59121100$

$\mathrm{N} \quad \quad \quad-1.55231500 \quad 1.89320600 \quad-1.04572800$

$\begin{array}{lllll}\mathrm{O} & -2.22700100 & 0.95479600 & -1.60263100\end{array}$ 


\section{TS, 2}

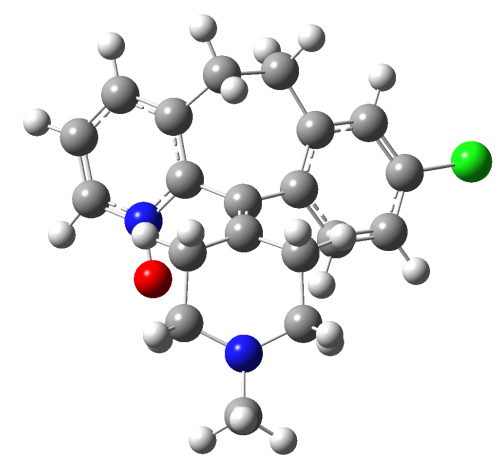

$\mathrm{Cl} \quad 5.21306700 \quad-1.71404000 \quad-0.68580600$

$\begin{array}{llll}\text { C } & 0.99682600 & -0.21147300 & 0.13383700\end{array}$

C $\quad 1.22433900 \quad-1.34947600 \quad-0.65460600$

$\mathrm{H} \quad \begin{array}{lllll}\mathrm{C} & 0.37077400 & -1.85267500 & -1.08549800\end{array}$

C $\quad 2.49810100 \quad-1.82690800 \quad-0.91429100$

$\mathrm{H} \quad 2.65125700 \quad-2.70404100 \quad-1.52478200$

C $\quad 3.57866100 \quad-1.13845900 \quad-0.38186200$

$\begin{array}{lllll}\mathrm{C} & 3.38910400 & 0.00252500 & 0.37649300\end{array}$

$\begin{array}{llll}\mathrm{H} & 4.25037300 & 0.52440400 & 0.77072800\end{array}$

$\begin{array}{lllll}\text { C } & 2.10507900 & 0.48944700 & 0.64244900\end{array}$

$\begin{array}{llll}\text { C } & -1.37281700 & -0.57437600 & 0.84103400\end{array}$

$\begin{array}{lllll}\text { C } & -1.16025100 & -1.95011300 & 1.40442200\end{array}$

$\mathrm{H} \quad-0.11230400 \quad-2.23396000 \quad 1.44674900$

$\mathrm{H} \quad-1.53254000 \quad-1.93201900 \quad 2.43320200$

$\begin{array}{llll}\text { C } & -1.94526200 & -3.01142800 & 0.61144300\end{array}$

$\mathrm{H} \quad-1.27849300 \quad-3.42605200 \quad-0.15843700$

$\mathrm{H} \quad-2.17767200 \quad-3.84630900 \quad 1.28888200$

$\mathrm{N} \quad-3.14913600 \quad-2.51520900 \quad-0.02080900$

C $\quad-3.66985700 \quad-1.15951300 \quad 0.06515400$

$\mathrm{H} \quad-4.69209800 \quad-1.17261000 \quad 0.46853600$

$\mathrm{H} \quad-3.72101800 \quad-0.72917500 \quad-0.93875400$

$\begin{array}{llll}\text { C } & -2.82355400 & -0.20618200 & 0.93106200\end{array}$

$\mathrm{H} \quad-3.13107400 \quad-0.29666100 \quad 1.97744400$

$\mathrm{H} \quad-3.02426200 \quad 0.81486700 \quad 0.61754600$

$\begin{array}{lllll}\mathrm{O} & -1.95439700 & 0.87510700 & -1.88636000\end{array}$

$\mathrm{N} \quad \quad-1.44781300 \quad 1.84297800 \quad-1.21463000$

C $\quad-0.69951200 \quad 1.61265000 \quad-0.08307100$

$\begin{array}{llll}\text { C } & -0.16436500 & 2.67796700 & 0.63836200\end{array}$

$\begin{array}{llll}\text { C } & -0.38450900 & 3.98519600 & 0.22075400\end{array}$

$\begin{array}{llll}\mathrm{H} & 0.02307100 & 4.81573100 & 0.77965300\end{array}$

$\begin{array}{lllll}\text { C } & -1.14263800 & 4.19965700 & -0.92623000\end{array}$

$\mathrm{H} \quad \begin{array}{lllll}\mathrm{H} & -1.34026100 & 5.19800700 & -1.28912100\end{array}$

C $\quad-1.65882300 \quad 3.13226600 \quad-1.62215900$

$\mathrm{H} \quad-2.25277100 \quad 3.20990000 \quad-2.51739600$

C

$\begin{array}{lrrr}\mathrm{C} & 2.04696200 & 1.76497100 & 1.47314200 \\ \mathrm{H} & 2.60314000 & 2.53550600 & 0.93172600 \\ \mathrm{H} & 2.60227400 & 1.59089300 & 2.39799400 \\ \mathrm{C} & 0.67133800 & 2.33983800 & 1.84505900 \\ \mathrm{H} & 0.82910300 & 3.23856200 & 2.44182400 \\ \mathrm{H} & 0.13304100 & 1.62775900 & 2.47232100 \\ \mathrm{C} & -4.07477100 & -3.51626100 & -0.47722700 \\ \mathrm{H} & -3.53774600 & -4.36296000 & -0.91167300 \\ \mathrm{H} & -4.72511600 & -3.90965800 & 0.32364100 \\ \mathrm{H} & -4.71980200 & -3.10845600 & -1.25802300\end{array}$

9 TS, 3

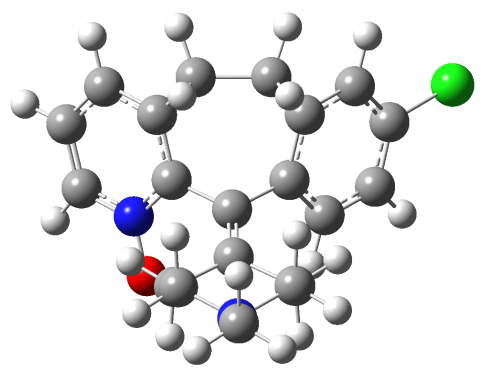

$\mathrm{Cl} \quad 5.28213600 \quad-1.77902900 \quad-0.15966000$

$\begin{array}{lllll}\mathrm{O} & -0.95213600 & 1.95244700 & -2.31757000\end{array}$

$\mathrm{N} \quad-0.59874800 \quad 2.43362800 \quad-1.18229600$

$\begin{array}{lllll}\text { C } & -0.38360400 & 1.61254200 & -0.09618200\end{array}$

$\begin{array}{lllll}\text { C } & -0.42005800 & 0.14752900 & -0.31759900\end{array}$

$\begin{array}{lllll}\text { C } & 0.95731100 & -0.41782100 & -0.25990900\end{array}$

$\begin{array}{lllll}\text { C } & 1.59187100 & -0.84494800 & -1.42583500\end{array}$

$\mathrm{H} \quad \begin{array}{llll}\mathrm{C} & 1.04451800 & -0.81925200 & -2.35777700\end{array}$

C $\quad 2.91388000-1.27480000-1.40956200$

$\mathrm{H} \quad 3.40281700 \quad-1.60404700 \quad-2.31412700$

C $\quad 3.60274200 \quad-1.25473500 \quad-0.20701900$

$\begin{array}{llll}\text { C } & 2.99381200 & -0.82767900 & 0.96566200\end{array}$

$\mathrm{H} \quad 3.55495500 \quad-0.83384400 \quad 1.88938800$

C $\quad 1.66441200 \quad-0.41058800 \quad 0.95294300$

$\begin{array}{llll}\text { C } & 0.97086200 & -0.06423600 & 2.25054900\end{array}$

$\mathrm{H} \quad 1.71878300 \quad-0.03792900 \quad 3.04178700$

$\mathrm{H} \quad 0.31139500 \quad-0.90122500 \quad 2.48957100$

C $\quad \begin{array}{llll}0.11902900 & 1.24737300 & 2.33937200\end{array}$

$\mathrm{H} \quad-0.89854600 \quad 0.96534100 \quad 2.61719500$

$\mathrm{H} \quad 0.49984300 \quad 1.84709000 \quad 3.16481700$

$\begin{array}{llll}\text { C } & 0.00313900 & 2.15078500 & 1.13257100\end{array}$

$\begin{array}{llll}\text { C } & 0.18429700 & 3.52425100 & 1.25516700\end{array}$

$\mathrm{H} \quad 0.48185200 \quad 3.95276900 \quad 2.20169800$

$\begin{array}{lllll}\mathrm{C} & -0.03681300 & 4.33762700 & 0.14825300\end{array}$

$\mathrm{H} \quad 0.09203100 \quad 5.40879900 \quad 0.20605600$

C $\quad-0.42942700 \quad 3.78397600 \quad-1.04604300$ 


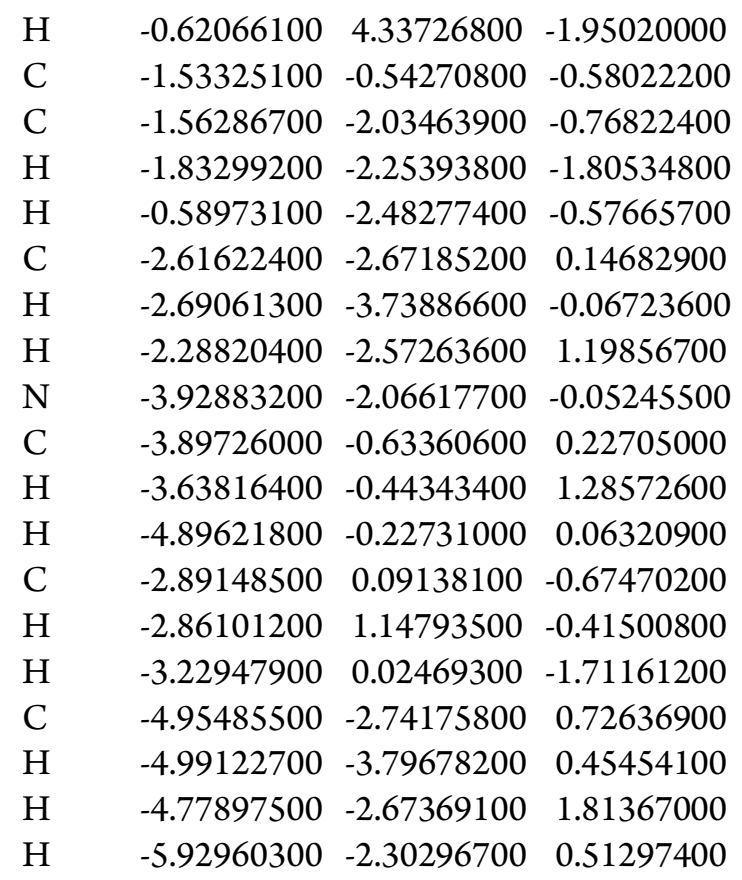

\section{TS, 4}

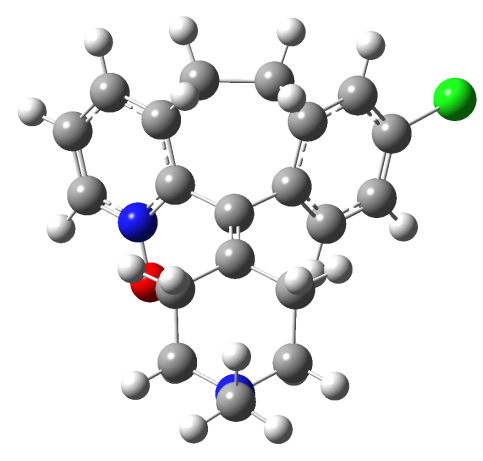

$\begin{array}{lrrrr}\mathrm{Cl} & & 5.15278600 & -1.89577200 & -0.59718200 \\ \mathrm{O} & -1.57057800 & 1.25709500 & -2.01748900 \\ \mathrm{~N} & -0.94206100 & 2.04648400 & -1.22234400 \\ \mathrm{C} & -0.41809300 & 1.59037000 & -0.03181000 \\ \mathrm{C} & -0.45732500 & 0.13197200 & 0.23085200 \\ \mathrm{C} & 0.90673200 & -0.44906000 & 0.07978700\end{array}$

$\begin{array}{llll}\mathrm{C} & 1.21943800 & -1.23555900 & -1.02893300 \\ \mathrm{H} & 0.43926100 & -1.46923400 & -1.73986900 \\ \mathrm{C} & 2.51345400 & -1.69577300 & -1.24461900 \\ \mathrm{H} & 2.75137700 & -2.30409900 & -2.10429800 \\ \mathrm{C} & 3.50247000 & -1.34046000 & -0.34102700 \\ \mathrm{C} & 3.21776300 & -0.55137400 & 0.76560500 \\ \mathrm{H} & 4.00930100 & -0.29909400 & 1.45700700 \\ \mathrm{C} & 1.91788600 & -0.10283500 & 0.99043900 \\ \mathrm{C} & 1.59022800 & 0.65510100 & 2.25640100 \\ \mathrm{H} & 2.52315600 & 0.88491800 & 2.76926600 \\ \mathrm{H} & 1.05230300 & -0.03586900 & 2.90851100 \\ \mathrm{C} & 0.74634900 & 1.97176400 & 2.16388900 \\ \mathrm{H} & -0.14304800 & 1.84224400 & 2.78350100 \\ \mathrm{H} & 1.31726900 & 2.77701900 & 2.62404100 \\ \mathrm{C} & 0.26890000 & 2.46232600 & 0.81578300 \\ \mathrm{C} & 0.42971000 & 3.79428700 & 0.44971500 \\ \mathrm{H} & 0.95836000 & 4.47791000 & 1.09882500 \\ \mathrm{C} & -0.10911000 & 4.23616000 & -0.75431700 \\ \mathrm{H} & -0.00584800 & 5.26403900 & -1.07065300 \\ \mathrm{C} & -0.78892600 & 3.36072500 & -1.56568200 \\ \mathrm{H} & -1.23404300 & 3.61529800 & -2.51292300 \\ \mathrm{C} & -1.57122700 & -0.55304400 & 0.51415200 \\ \mathrm{C} & -1.60369000 & -2.04092400 & 0.72971700 \\ \mathrm{H} & -0.61322800 & -2.48702100 & 0.66207400 \\ \mathrm{H} & -1.98949600 & -2.25107900 & 1.73180900 \\ \mathrm{C} & -2.55059400 & -2.68897400 & -0.31631900 \\ \mathrm{H} & -2.11101800 & -2.55587700 & -1.30774900 \\ \mathrm{H} & -2.62839500 & -3.76153500 & -0.13403800 \\ \mathrm{~N} & -3.89038500 & -2.11084900 & -0.33597900 \\ \mathrm{C} & -3.82718300 & -0.66182300 & -0.52174500 \\ \mathrm{H} & -4.84003700 & -0.25858000 & -0.49727400 \\ \mathrm{H} & -3.40918900 & -0.45228900 & -1.50616800 \\ \mathrm{C} & -2.94169800 & 0.06414900 & 0.52683900 \\ \mathrm{H} & -3.38642300 & -0.05449300 & 1.51967800 \\ \mathrm{H} & -2.91470600 & 1.12828700 & 0.30235100 \\ \mathrm{C} & -4.72572000 & -2.52816800 & 0.78186900 \\ \mathrm{H} & -4.79695300 & -3.61622900 & 0.79359500 \\ \mathrm{H} & -4.36894600 & -2.20594000 & 1.77198700 \\ \mathrm{H} & -5.73149500 & -2.12961400 & 0.64701100\end{array}$




\section{Crystallographic Data}

\section{i. Experimental}

Low-temperature diffraction data ( $\omega$-scans) were collected on a Rigaku MicroMax-007HF diffractometer coupled to a Saturn994+CCD detector with $\mathrm{Cu} \mathrm{Ka}(\lambda=1.54178 \AA)$ for the structure of $( \pm)-6 \mathrm{~b}$. The diffraction images were processed and scaled using Rigaku Oxford Diffraction software. ${ }^{10}$ The structure was solved with SHELXT and was refined against $\mathrm{F}^{2}$ on all data by full-matrix least squares with SHELXL. ${ }^{11}$ All non-hydrogen atoms were refined anisotropically. Hydrogen atoms were included in the model at geometrically calculated positions and refined using a riding model. The isotropic displacement parameters of all hydrogen atoms were fixed to 1.2 times the $U$ value of the atoms to which they are linked (1.5 times for methyl groups).

For $( \pm)-6 \mathbf{b}$, the only exception is $\mathrm{H} 3$, which was found in the difference map and freely refined. For (-)-6f, the program SQUEEZE was used to compensate for the contribution of disordered solvents contained in voids within the crystal lattice from the diffraction intensities. This procedure was applied to the data file and the submitted model is based on the solvent removed data. Based on the total electron density found in the voids $\left(33 \mathrm{e} / \AA^{3}\right)$, it is likely that $\sim 1.5$ ethanol molecules are present in the unit cell. See "_platon_squeeze_details" in this .cif for more information. For $\mathbf{8 h}, \mathrm{H} 3 \mathrm{H} 4 \mathrm{a}$ and $\mathrm{H} 5 \mathrm{a}$ were found in the difference map and freely refined.

The full numbering scheme of compound $( \pm)-6 \mathbf{b},(-)-6 \mathbf{f}$, and $8 \mathbf{h}$ can be found in the X-ray structure determination (CIF), which is included as supporting information. The CCDC numbers for ( \pm )-6b (1983136), (-)-6f (1983464), and $\mathbf{8 h}$ (1983156) contain the supplementary crystallographic data for this paper. These data can be obtained free of charge from The Cambridge Crystallographic Data Center via www.ccdc.cam.ac.uk/data_request/cif.

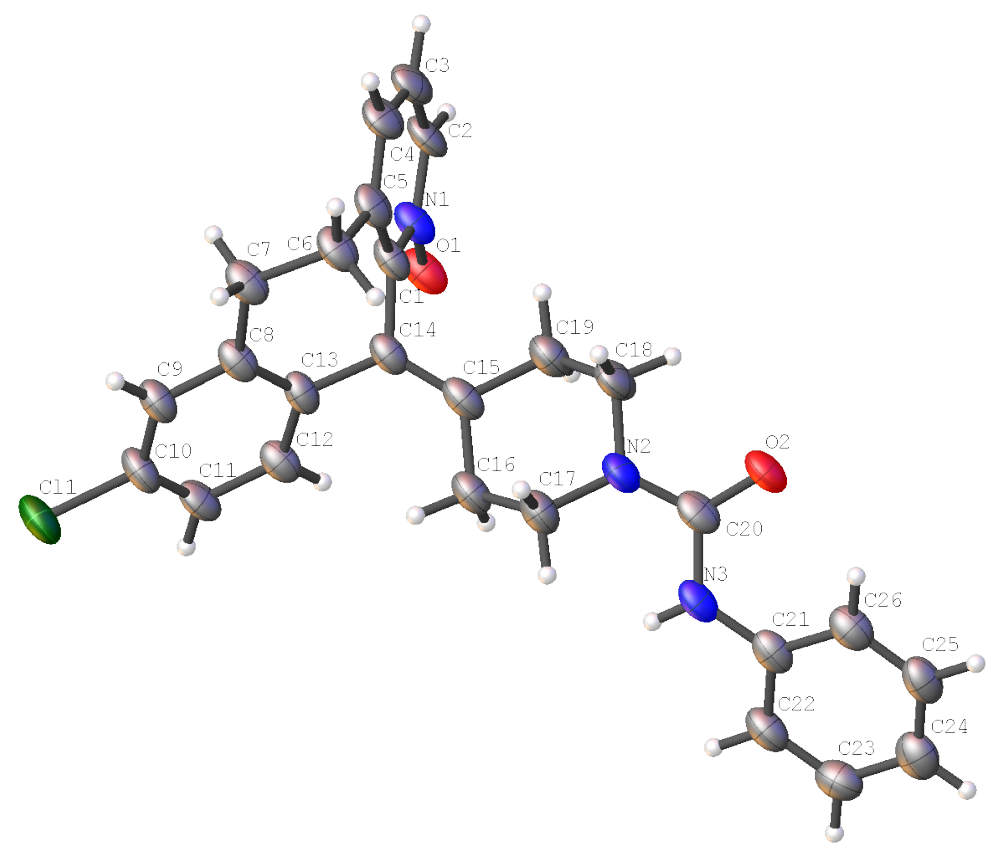

Figure S6. The complete numbering scheme of $( \pm)-6 \mathrm{~b}$ with $50 \%$ thermal ellipsoid probability levels. The hydrogen atoms are shown as circles for clarity. 
Table S6. Crystal data and structure refinement for ( \pm )-6b.

Identification code

Empirical formula

Formula weight

Temperature

Wavelength

Crystal system

Space group

Unit cell dimensions

Volume

Z

Density (calculated)

Absorption coefficient

$\mathrm{F}(000)$

Crystal size

Crystal color and habit

Diffractometer

Theta range for data collection

Index ranges

Reflections collected

Independent reflections

Observed reflections $(\mathrm{I}>2 \operatorname{sigma}(\mathrm{I}))$

Completeness to theta $=66.597^{\circ}$

Absorption correction

Max. and min. transmission

Solution method

Refinement method

Data / restraints / parameters

Goodness-of-fit on $\mathrm{F}^{2}$

Final R indices $[\mathrm{I}>2 \operatorname{sigma}(\mathrm{I})]$

Rindices (all data)

Largest diff. peak and hole
1983136

$\mathrm{C}_{26} \mathrm{H}_{24} \mathrm{ClN}_{3} \mathrm{O}_{2}$

445.93

93(2) K

$1.54184 \AA$

Monoclinic

$\mathrm{P} 21 / \mathrm{n}$

$\mathrm{a}=10.2011(9) \AA$

$\alpha=90^{\circ}$

$\mathrm{b}=19.0900(9) \AA$

$\beta=101.312(8)^{\circ}$

$c=11.4719(8) \AA$

$\gamma=90^{\circ}$

2190.6(3) $\AA^{3}$

4

$1.352 \mathrm{Mg} / \mathrm{m}^{3}$

$1.775 \mathrm{~mm}^{-1}$

936

$0.200 \times 0.040 \times 0.010 \mathrm{~mm}^{3}$

White

Rigaku Saturn 944+ CCD

4.562 to $66.597^{\circ}$

$-12<=\mathrm{h}<=12,-22<=\mathrm{k}<=22,-13<=\mathrm{l}<=13$

68070

$3868[\mathrm{R}(\mathrm{int})=0.2246]$

2720

$100.0 \%$

Semi-empirical from equivalents

1.00000 and 0.34394

SHELXT-2014/5 (Sheldrick, 2014)

SHELXL-2014/7 (Sheldrick, 2014)

3868 / 0 / 293

1.096

$\mathrm{R} 1=0.0818, \mathrm{wR} 2=0.1831$

$\mathrm{R} 1=0.1169, \mathrm{wR} 2=0.2036$

0.333 and -0.449 e. $\AA^{-3}$ 
Table S7. Atomic coordinates $\left(\mathrm{x} 10^{4}\right)$ and equivalent isotropic displacement parameters $\left(\AA^{2} \times 10^{3}\right)$ for $( \pm)$-6b. $\mathrm{U}(\mathrm{eq})$ is defined as one third of the trace of the orthogonalized $\mathrm{U}^{\mathrm{ij}}$ tensor.

\begin{tabular}{|c|c|c|c|c|}
\hline & $\mathrm{x}$ & $\mathrm{y}$ & $\mathrm{z}$ & $\mathrm{U}(\mathrm{eq})$ \\
\hline $\mathrm{Cl}(1)$ & $-3410(1)$ & 397(1) & $8176(1)$ & $56(1)$ \\
\hline $\mathrm{O}(1)$ & $-522(3)$ & $2811(1)$ & $4034(3)$ & $49(1)$ \\
\hline $\mathrm{O}(2)$ & $4388(3)$ & $4572(1)$ & $7869(3)$ & $48(1)$ \\
\hline $\mathrm{N}(1)$ & $-1403(4)$ & $3266(2)$ & $4266(3)$ & $42(1)$ \\
\hline $\mathrm{N}(2)$ & $2649(4)$ & $3878(2)$ & $8089(3)$ & $46(1)$ \\
\hline $\mathrm{N}(3)$ & $4771(4)$ & $3688(2)$ & $9229(3)$ & $42(1)$ \\
\hline$C(1)$ & $-1682(5)$ & $3310(2)$ & $5380(4)$ & $39(1)$ \\
\hline$C(2)$ & $-2044(5)$ & $3690(2)$ & $3380(4)$ & $45(1)$ \\
\hline$C(3)$ & $-3022(5)$ & $4131(2)$ & $3568(4)$ & $48(1)$ \\
\hline $\mathrm{C}(4)$ & $-3389(5)$ & $4154(2)$ & $4667(4)$ & $48(1)$ \\
\hline$C(5)$ & $-2711(5)$ & $3745(2)$ & $5580(4)$ & $44(1)$ \\
\hline$C(6)$ & $-3083(5)$ & $3700(2)$ & $6779(4)$ & $45(1)$ \\
\hline$C(7)$ & $-3722(5)$ & $2988(2)$ & $6920(4)$ & $45(1)$ \\
\hline$C(8)$ & $-2821(5)$ & $2344(2)$ & $7075(4)$ & $39(1)$ \\
\hline$C(9)$ & $-3378(5)$ & $1749(2)$ & $7502(4)$ & $42(1)$ \\
\hline$C(10)$ & $-2679(5)$ & $1124(2)$ & $7629(4)$ & $44(1)$ \\
\hline$C(11)$ & $-1450(5)$ & $1057(2)$ & $7328(4)$ & $43(1)$ \\
\hline$C(12)$ & $-882(5)$ & $1653(2)$ & $6929(4)$ & $40(1)$ \\
\hline$C(13)$ & $-1533(4)$ & $2301(2)$ & $6816(4)$ & $38(1)$ \\
\hline$C(14)$ & $-857(5)$ & $2905(2)$ & $6360(4)$ & $37(1)$ \\
\hline$C(15)$ & $410(5)$ & $3110(2)$ & $6792(4)$ & $42(1)$ \\
\hline$C(16)$ & $1312(5)$ & $2808(2)$ & $7858(4)$ & $44(1)$ \\
\hline$C(17)$ & $1970(5)$ & $3369(2)$ & $8704(4)$ & $46(1)$ \\
\hline$C(18)$ & $1748(5)$ & $4222(2)$ & $7118(4)$ & $51(1)$ \\
\hline$C(19)$ & $1077(5)$ & $3692(2)$ & $6222(4)$ & $48(1)$ \\
\hline$C(20)$ & $3946(5)$ & $4074(2)$ & $8362(4)$ & $43(1)$ \\
\hline$C(21)$ & $6135(5)$ & $3824(2)$ & $9624(4)$ & $41(1)$ \\
\hline$C(22)$ & $7009(5)$ & $3262(2)$ & $9921(4)$ & $47(1)$ \\
\hline$C(23)$ & $8357(5)$ & $3376(2)$ & $10299(4)$ & $50(1)$ \\
\hline$C(24)$ & $8873(5)$ & $4049(2)$ & $10395(4)$ & $51(1)$ \\
\hline$C(25)$ & $8010(5)$ & $4608(2)$ & $10139(4)$ & $48(1)$ \\
\hline$C(26)$ & $6655(5)$ & $4505(2)$ & $9757(4)$ & $46(1)$ \\
\hline
\end{tabular}


Table S8. Bond lengths $[\AA]$ and angles $\left[^{\circ}\right]$ for $( \pm)-6 \mathbf{b}$.

\begin{tabular}{|c|c|}
\hline $\mathrm{Cl}(1)-\mathrm{C}(10)$ & $1.750(4)$ \\
\hline $\mathrm{O}(1)-\mathrm{N}(1)$ & $1.315(4)$ \\
\hline $\mathrm{O}(2)-\mathrm{C}(20)$ & $1.236(5)$ \\
\hline $\mathrm{N}(1)-\mathrm{C}(2)$ & $1.361(5)$ \\
\hline $\mathrm{N}(1)-\mathrm{C}(1)$ & $1.365(5)$ \\
\hline $\mathrm{N}(2)-\mathrm{C}(20)$ & $1.352(6)$ \\
\hline $\mathrm{N}(2)-\mathrm{C}(17)$ & $1.453(5)$ \\
\hline $\mathrm{N}(2)-\mathrm{C}(18)$ & $1.455(6)$ \\
\hline $\mathrm{N}(3)-\mathrm{C}(20)$ & $1.383(6)$ \\
\hline $\mathrm{N}(3)-\mathrm{C}(21)$ & $1.402(6)$ \\
\hline $\mathrm{N}(3)-\mathrm{H}(3)$ & $0.83(4)$ \\
\hline$C(1)-C(5)$ & $1.392(6)$ \\
\hline$C(1)-C(14)$ & $1.483(6)$ \\
\hline$C(2)-C(3)$ & $1.356(6)$ \\
\hline $\mathrm{C}(2)-\mathrm{H}(2)$ & 0.9500 \\
\hline$C(3)-C(4)$ & $1.385(6)$ \\
\hline $\mathrm{C}(3)-\mathrm{H}(3 \mathrm{~A})$ & 0.9500 \\
\hline$C(4)-C(5)$ & $1.379(6)$ \\
\hline $\mathrm{C}(4)-\mathrm{H}(4)$ & 0.9500 \\
\hline$C(5)-C(6)$ & $1.499(6)$ \\
\hline$C(6)-C(7)$ & $1.531(6)$ \\
\hline $\mathrm{C}(6)-\mathrm{H}(6 \mathrm{~A})$ & 0.9900 \\
\hline $\mathrm{C}(6)-\mathrm{H}(6 \mathrm{~B})$ & 0.9900 \\
\hline$C(7)-C(8)$ & $1.524(6)$ \\
\hline $\mathrm{C}(7)-\mathrm{H}(7 \mathrm{~A})$ & 0.9900 \\
\hline $\mathrm{C}(7)-\mathrm{H}(7 \mathrm{~B})$ & 0.9900 \\
\hline$C(8)-C(9)$ & $1.401(6)$ \\
\hline$C(8)-C(13)$ & $1.406(6)$ \\
\hline$C(9)-C(10)$ & $1.382(6)$ \\
\hline $\mathrm{C}(9)-\mathrm{H}(9)$ & 0.9500 \\
\hline$C(10)-C(11)$ & $1.371(6)$ \\
\hline$C(11)-C(12)$ & $1.393(6)$ \\
\hline $\mathrm{C}(11)-\mathrm{H}(11)$ & 0.9500 \\
\hline$C(12)-C(13)$ & $1.397(6)$ \\
\hline $\mathrm{C}(12)-\mathrm{H}(12)$ & 0.9500 \\
\hline
\end{tabular}




\begin{tabular}{|c|c|}
\hline$C(13)-C(14)$ & $1.490(5)$ \\
\hline$C(14)-C(15)$ & $1.349(6)$ \\
\hline$C(15)-C(16)$ & $1.495(6)$ \\
\hline$C(15)-C(19)$ & $1.517(6)$ \\
\hline$C(16)-C(17)$ & $1.512(6)$ \\
\hline $\mathrm{C}(16)-\mathrm{H}(16 \mathrm{~A})$ & 0.9900 \\
\hline $\mathrm{C}(16)-\mathrm{H}(16 \mathrm{~B})$ & 0.9900 \\
\hline $\mathrm{C}(17)-\mathrm{H}(17 \mathrm{~A})$ & 0.9900 \\
\hline $\mathrm{C}(17)-\mathrm{H}(17 \mathrm{~B})$ & 0.9900 \\
\hline$C(18)-C(19)$ & $1.508(6)$ \\
\hline $\mathrm{C}(18)-\mathrm{H}(18 \mathrm{~A})$ & 0.9900 \\
\hline $\mathrm{C}(18)-\mathrm{H}(18 \mathrm{~B})$ & 0.9900 \\
\hline $\mathrm{C}(19)-\mathrm{H}(19 \mathrm{~A})$ & 0.9900 \\
\hline $\mathrm{C}(19)-\mathrm{H}(19 \mathrm{~B})$ & 0.9900 \\
\hline$C(21)-C(22)$ & $1.394(6)$ \\
\hline$C(21)-C(26)$ & $1.400(6)$ \\
\hline$C(22)-C(23)$ & $1.376(7)$ \\
\hline $\mathrm{C}(22)-\mathrm{H}(22)$ & 0.9500 \\
\hline$C(23)-C(24)$ & $1.383(6)$ \\
\hline $\mathrm{C}(23)-\mathrm{H}(23)$ & 0.9500 \\
\hline$C(24)-C(25)$ & $1.378(7)$ \\
\hline $\mathrm{C}(24)-\mathrm{H}(24)$ & 0.9500 \\
\hline$C(25)-C(26)$ & $1.380(7)$ \\
\hline $\mathrm{C}(25)-\mathrm{H}(25)$ & 0.9500 \\
\hline $\mathrm{C}(26)-\mathrm{H}(26)$ & 0.9500 \\
\hline $\mathrm{O}(1)-\mathrm{N}(1)-\mathrm{C}(2)$ & $119.2(3)$ \\
\hline $\mathrm{O}(1)-\mathrm{N}(1)-\mathrm{C}(1)$ & $120.4(3)$ \\
\hline $\mathrm{C}(2)-\mathrm{N}(1)-\mathrm{C}(1)$ & $120.3(4)$ \\
\hline $\mathrm{C}(20)-\mathrm{N}(2)-\mathrm{C}(17)$ & $127.6(4)$ \\
\hline $\mathrm{C}(20)-\mathrm{N}(2)-\mathrm{C}(18)$ & $119.7(4)$ \\
\hline $\mathrm{C}(17)-\mathrm{N}(2)-\mathrm{C}(18)$ & $112.6(4)$ \\
\hline $\mathrm{C}(20)-\mathrm{N}(3)-\mathrm{C}(21)$ & $124.0(4)$ \\
\hline $\mathrm{C}(20)-\mathrm{N}(3)-\mathrm{H}(3)$ & $112(3)$ \\
\hline $\mathrm{C}(21)-\mathrm{N}(3)-\mathrm{H}(3)$ & $117(3)$ \\
\hline $\mathrm{N}(1)-\mathrm{C}(1)-\mathrm{C}(5)$ & $119.6(4)$ \\
\hline
\end{tabular}




\begin{tabular}{|c|c|}
\hline $\mathrm{N}(1)-\mathrm{C}(1)-\mathrm{C}(14)$ & $119.1(4)$ \\
\hline$C(5)-C(1)-C(14)$ & $121.3(4)$ \\
\hline$C(3)-C(2)-N(1)$ & $120.7(4)$ \\
\hline $\mathrm{C}(3)-\mathrm{C}(2)-\mathrm{H}(2)$ & 119.6 \\
\hline $\mathrm{N}(1)-\mathrm{C}(2)-\mathrm{H}(2)$ & 119.6 \\
\hline$C(2)-C(3)-C(4)$ & $120.2(4)$ \\
\hline $\mathrm{C}(2)-\mathrm{C}(3)-\mathrm{H}(3 \mathrm{~A})$ & 119.9 \\
\hline $\mathrm{C}(4)-\mathrm{C}(3)-\mathrm{H}(3 \mathrm{~A})$ & 119.9 \\
\hline$C(5)-C(4)-C(3)$ & $119.3(4)$ \\
\hline $\mathrm{C}(5)-\mathrm{C}(4)-\mathrm{H}(4)$ & 120.4 \\
\hline $\mathrm{C}(3)-\mathrm{C}(4)-\mathrm{H}(4)$ & 120.4 \\
\hline$C(4)-C(5)-C(1)$ & $119.6(4)$ \\
\hline$C(4)-C(5)-C(6)$ & $123.3(4)$ \\
\hline$C(1)-C(5)-C(6)$ & $116.9(4)$ \\
\hline$C(5)-C(6)-C(7)$ & $109.7(3)$ \\
\hline$C(5)-C(6)-H(6 A)$ & 109.7 \\
\hline$C(7)-C(6)-H(6 A)$ & 109.7 \\
\hline $\mathrm{C}(5)-\mathrm{C}(6)-\mathrm{H}(6 \mathrm{~B})$ & 109.7 \\
\hline $\mathrm{C}(7)-\mathrm{C}(6)-\mathrm{H}(6 \mathrm{~B})$ & 109.7 \\
\hline$H(6 A)-C(6)-H(6 B)$ & 108.2 \\
\hline$C(8)-C(7)-C(6)$ & $118.0(4)$ \\
\hline $\mathrm{C}(8)-\mathrm{C}(7)-\mathrm{H}(7 \mathrm{~A})$ & 107.8 \\
\hline$C(6)-C(7)-H(7 A)$ & 107.8 \\
\hline $\mathrm{C}(8)-\mathrm{C}(7)-\mathrm{H}(7 \mathrm{~B})$ & 107.8 \\
\hline $\mathrm{C}(6)-\mathrm{C}(7)-\mathrm{H}(7 \mathrm{~B})$ & 107.8 \\
\hline $\mathrm{H}(7 \mathrm{~A})-\mathrm{C}(7)-\mathrm{H}(7 \mathrm{~B})$ & 107.1 \\
\hline$C(9)-C(8)-C(13)$ & $119.1(4)$ \\
\hline$C(9)-C(8)-C(7)$ & $114.7(4)$ \\
\hline$C(13)-C(8)-C(7)$ & $126.1(4)$ \\
\hline$C(10)-C(9)-C(8)$ & $120.0(4)$ \\
\hline $\mathrm{C}(10)-\mathrm{C}(9)-\mathrm{H}(9)$ & 120.0 \\
\hline $\mathrm{C}(8)-\mathrm{C}(9)-\mathrm{H}(9)$ & 120.0 \\
\hline$C(11)-C(10)-C(9)$ & $122.2(4)$ \\
\hline$C(11)-C(10)-C l(1)$ & $119.4(3)$ \\
\hline $\mathrm{C}(9)-\mathrm{C}(10)-\mathrm{Cl}(1)$ & $118.4(3)$ \\
\hline$C(10)-C(11)-C(12)$ & $117.8(4)$ \\
\hline
\end{tabular}




\begin{tabular}{|c|c|}
\hline $\mathrm{C}(10)-\mathrm{C}(11)-\mathrm{H}(11)$ & 121.1 \\
\hline $\mathrm{C}(12)-\mathrm{C}(11)-\mathrm{H}(11)$ & 121.1 \\
\hline$C(11)-C(12)-C(13)$ & $122.2(4)$ \\
\hline $\mathrm{C}(11)-\mathrm{C}(12)-\mathrm{H}(12)$ & 118.9 \\
\hline $\mathrm{C}(13)-\mathrm{C}(12)-\mathrm{H}(12)$ & 118.9 \\
\hline$C(12)-C(13)-C(8)$ & $118.6(4)$ \\
\hline$C(12)-C(13)-C(14)$ & $118.3(4)$ \\
\hline$C(8)-C(13)-C(14)$ & $123.0(4)$ \\
\hline$C(15)-C(14)-C(1)$ & $119.2(4)$ \\
\hline$C(15)-C(14)-C(13)$ & $124.9(4)$ \\
\hline$C(1)-C(14)-C(13)$ & $116.0(4)$ \\
\hline$C(14)-C(15)-C(16)$ & $125.5(4)$ \\
\hline$C(14)-C(15)-C(19)$ & $122.2(4)$ \\
\hline$C(16)-C(15)-C(19)$ & $112.3(4)$ \\
\hline$C(15)-C(16)-C(17)$ & $112.1(3)$ \\
\hline$C(15)-C(16)-H(16 A)$ & 109.2 \\
\hline $\mathrm{C}(17)-\mathrm{C}(16)-\mathrm{H}(16 \mathrm{~A})$ & 109.2 \\
\hline$C(15)-C(16)-H(16 B)$ & 109.2 \\
\hline $\mathrm{C}(17)-\mathrm{C}(16)-\mathrm{H}(16 \mathrm{~B})$ & 109.2 \\
\hline $\mathrm{H}(16 \mathrm{~A})-\mathrm{C}(16)-\mathrm{H}(16 \mathrm{~B})$ & 107.9 \\
\hline $\mathrm{N}(2)-\mathrm{C}(17)-\mathrm{C}(16)$ & $110.9(4)$ \\
\hline $\mathrm{N}(2)-\mathrm{C}(17)-\mathrm{H}(17 \mathrm{~A})$ & 109.5 \\
\hline $\mathrm{C}(16)-\mathrm{C}(17)-\mathrm{H}(17 \mathrm{~A})$ & 109.5 \\
\hline $\mathrm{N}(2)-\mathrm{C}(17)-\mathrm{H}(17 \mathrm{~B})$ & 109.5 \\
\hline $\mathrm{C}(16)-\mathrm{C}(17)-\mathrm{H}(17 \mathrm{~B})$ & 109.5 \\
\hline $\mathrm{H}(17 \mathrm{~A})-\mathrm{C}(17)-\mathrm{H}(17 \mathrm{~B})$ & 108.1 \\
\hline $\mathrm{N}(2)-\mathrm{C}(18)-\mathrm{C}(19)$ & $110.7(4)$ \\
\hline $\mathrm{N}(2)-\mathrm{C}(18)-\mathrm{H}(18 \mathrm{~A})$ & 109.5 \\
\hline $\mathrm{C}(19)-\mathrm{C}(18)-\mathrm{H}(18 \mathrm{~A})$ & 109.5 \\
\hline $\mathrm{N}(2)-\mathrm{C}(18)-\mathrm{H}(18 \mathrm{~B})$ & 109.5 \\
\hline $\mathrm{C}(19)-\mathrm{C}(18)-\mathrm{H}(18 \mathrm{~B})$ & 109.5 \\
\hline $\mathrm{H}(18 \mathrm{~A})-\mathrm{C}(18)-\mathrm{H}(18 \mathrm{~B})$ & 108.1 \\
\hline$C(18)-C(19)-C(15)$ & $112.3(4)$ \\
\hline $\mathrm{C}(18)-\mathrm{C}(19)-\mathrm{H}(19 \mathrm{~A})$ & 109.1 \\
\hline$C(15)-C(19)-H(19 A)$ & 109.1 \\
\hline $\mathrm{C}(18)-\mathrm{C}(19)-\mathrm{H}(19 \mathrm{~B})$ & 109.1 \\
\hline
\end{tabular}




$\begin{array}{ll}\mathrm{C}(15)-\mathrm{C}(19)-\mathrm{H}(19 B) & 109.1 \\ \mathrm{H}(19 \mathrm{~A})-\mathrm{C}(19)-\mathrm{H}(19 \mathrm{~B}) & 107.9 \\ \mathrm{O}(2)-\mathrm{C}(20)-\mathrm{N}(2) & 122.4(4) \\ \mathrm{O}(2)-\mathrm{C}(20)-\mathrm{N}(3) & 121.0(4) \\ \mathrm{N}(2)-\mathrm{C}(20)-\mathrm{N}(3) & 116.7(4) \\ \mathrm{C}(22)-\mathrm{C}(21)-\mathrm{C}(26) & 118.5(5) \\ \mathrm{C}(22)-\mathrm{C}(21)-\mathrm{N}(3) & 118.9(4) \\ \mathrm{C}(26)-\mathrm{C}(21)-\mathrm{N}(3) & 122.6(4) \\ \mathrm{C}(23)-\mathrm{C}(22)-\mathrm{C}(21) & 120.4(4) \\ \mathrm{C}(23)-\mathrm{C}(22)-\mathrm{H}(22) & 119.8 \\ \mathrm{C}(21)-\mathrm{C}(22)-\mathrm{H}(22) & 119.8 \\ \mathrm{C}(22)-\mathrm{C}(23)-\mathrm{C}(24) & 120.9(4) \\ \mathrm{C}(22)-\mathrm{C}(23)-\mathrm{H}(23) & 119.6 \\ \mathrm{C}(24)-\mathrm{C}(23)-\mathrm{H}(23) & 119.6 \\ \mathrm{C}(25)-\mathrm{C}(24)-\mathrm{C}(23) & 119.0(5) \\ \mathrm{C}(25)-\mathrm{C}(24)-\mathrm{H}(24) & 120.5 \\ \mathrm{C}(23)-\mathrm{C}(24)-\mathrm{H}(24) & 120.5 \\ \mathrm{C}(24)-\mathrm{C}(25)-\mathrm{C}(26) & 121.0(4) \\ \mathrm{C}(24)-\mathrm{C}(25)-\mathrm{H}(25) & 119.5 \\ \mathrm{C}(26)-\mathrm{C}(25)-\mathrm{H}(25) & 119.5 \\ \mathrm{C}(25)-\mathrm{C}(26)-\mathrm{C}(21) & 120.1(4) \\ \mathrm{C}(25)-\mathrm{C}(26)-\mathrm{H}(26) & 120.0 \\ \mathrm{C}(21)-\mathrm{C}(26)-\mathrm{H}(26) & 120.0 \\ & \\ & \end{array}$


Table S9. Anisotropic displacement parameters $\left(\AA^{2} \times 10^{3}\right)$ for $( \pm)-6 \mathrm{~b}$. The anisotropic displacement factor exponent takes the form: $-2 \pi^{2}\left[h^{2} a^{* 2} U^{11}+\ldots+2 h k a^{*} b^{*} U^{12}\right]$

\begin{tabular}{|c|c|c|c|c|c|c|}
\hline & $\mathrm{U}^{11}$ & $\mathrm{U}^{22}$ & $\mathrm{U}^{33}$ & $U^{23}$ & $\mathrm{U}^{13}$ & $\mathrm{U}^{12}$ \\
\hline $\mathrm{Cl}(1)$ & $76(1)$ & $29(1)$ & $69(1)$ & $11(1)$ & $31(1)$ & $-3(1)$ \\
\hline $\mathrm{O}(1)$ & $73(2)$ & $31(2)$ & $49(2)$ & $2(1)$ & $29(2)$ & $5(1)$ \\
\hline $\mathrm{O}(2)$ & $67(2)$ & $26(2)$ & $53(2)$ & $8(1)$ & $18(2)$ & $0(1)$ \\
\hline $\mathrm{N}(1)$ & $62(2)$ & $22(2)$ & $45(2)$ & $1(2)$ & $21(2)$ & $2(2)$ \\
\hline $\mathrm{N}(2)$ & $56(3)$ & $26(2)$ & $55(2)$ & $8(2)$ & $10(2)$ & $-3(2)$ \\
\hline $\mathrm{N}(3)$ & $62(3)$ & $21(2)$ & $44(2)$ & $0(2)$ & $17(2)$ & $-1(2)$ \\
\hline $\mathrm{C}(1)$ & $57(3)$ & $22(2)$ & $44(3)$ & $0(2)$ & $22(2)$ & $-3(2)$ \\
\hline$C(2)$ & $68(3)$ & $20(2)$ & $50(3)$ & $8(2)$ & $18(2)$ & $-2(2)$ \\
\hline$C(3)$ & $70(3)$ & $21(2)$ & $51(3)$ & $4(2)$ & $8(2)$ & $-3(2)$ \\
\hline$C(4)$ & $63(3)$ & $21(2)$ & $61(3)$ & $2(2)$ & $14(2)$ & $3(2)$ \\
\hline$C(5)$ & $61(3)$ & $20(2)$ & $51(3)$ & $-4(2)$ & $17(2)$ & $-5(2)$ \\
\hline$C(6)$ & $65(3)$ & $23(2)$ & $53(3)$ & $-3(2)$ & $23(2)$ & $2(2)$ \\
\hline$C(7)$ & $66(3)$ & $26(2)$ & $46(3)$ & $-4(2)$ & $23(2)$ & $3(2)$ \\
\hline$C(8)$ & $60(3)$ & $26(2)$ & $35(2)$ & $-4(2)$ & $19(2)$ & $0(2)$ \\
\hline $\mathrm{C}(9)$ & $59(3)$ & $27(2)$ & $45(3)$ & $1(2)$ & $22(2)$ & $-3(2)$ \\
\hline$C(10)$ & $64(3)$ & $24(2)$ & $51(3)$ & $3(2)$ & $27(2)$ & $-3(2)$ \\
\hline$C(11)$ & $67(3)$ & $21(2)$ & $44(3)$ & $0(2)$ & $15(2)$ & $2(2)$ \\
\hline$C(12)$ & $59(3)$ & $29(2)$ & $37(2)$ & $-2(2)$ & $19(2)$ & $1(2)$ \\
\hline$C(13)$ & $54(3)$ & $25(2)$ & $38(2)$ & $-3(2)$ & $18(2)$ & $-6(2)$ \\
\hline$C(14)$ & $55(3)$ & $24(2)$ & $37(2)$ & $-1(2)$ & $19(2)$ & $-1(2)$ \\
\hline$C(15)$ & $65(3)$ & $26(2)$ & $41(2)$ & $2(2)$ & $21(2)$ & $1(2)$ \\
\hline$C(16)$ & $61(3)$ & $28(2)$ & $46(3)$ & $4(2)$ & $19(2)$ & $-2(2)$ \\
\hline $\mathrm{C}(17)$ & $60(3)$ & $33(2)$ & $48(3)$ & $3(2)$ & $19(2)$ & $-5(2)$ \\
\hline$C(18)$ & $67(3)$ & $26(2)$ & $58(3)$ & $8(2)$ & $6(2)$ & $-4(2)$ \\
\hline$C(19)$ & $64(3)$ & $33(2)$ & $49(3)$ & $6(2)$ & $14(2)$ & $-4(2)$ \\
\hline$C(20)$ & $65(3)$ & $23(2)$ & $46(3)$ & $-3(2)$ & $20(2)$ & $5(2)$ \\
\hline$C(21)$ & $62(3)$ & $31(2)$ & $34(2)$ & $-2(2)$ & $20(2)$ & $-2(2)$ \\
\hline$C(22)$ & $67(3)$ & $29(2)$ & $49(3)$ & $3(2)$ & $24(2)$ & $1(2)$ \\
\hline$C(23)$ & $62(3)$ & $43(3)$ & $47(3)$ & $7(2)$ & $18(2)$ & $10(2)$ \\
\hline$C(24)$ & $67(3)$ & $48(3)$ & $43(3)$ & $-1(2)$ & $21(2)$ & $-6(2)$ \\
\hline$C(25)$ & $69(3)$ & $34(2)$ & $44(3)$ & $-4(2)$ & $17(2)$ & $-9(2)$ \\
\hline$C(26)$ & $65(3)$ & $34(2)$ & $43(3)$ & $-3(2)$ & $20(2)$ & $3(2)$ \\
\hline
\end{tabular}


Table S10. Hydrogen coordinates $\left(\times 10^{4}\right)$ and isotropic displacement parameters $\left(\AA^{2} \times 10^{3}\right)$ for $( \pm)-6 \mathbf{b}$.

\begin{tabular}{|c|c|c|c|c|}
\hline & $\mathrm{x}$ & $y$ & $\mathrm{z}$ & $\mathrm{U}(\mathrm{eq})$ \\
\hline $\mathrm{H}(2)$ & -1803 & 3675 & 2621 & 54 \\
\hline $\mathrm{H}(3 \mathrm{~A})$ & -3457 & 4426 & 2943 & 57 \\
\hline $\mathrm{H}(4)$ & -4099 & 4449 & 4791 & 57 \\
\hline $\mathrm{H}(6 \mathrm{~A})$ & -3720 & 4079 & 6864 & 54 \\
\hline $\mathrm{H}(6 \mathrm{~B})$ & -2274 & 3761 & 7408 & 54 \\
\hline $\mathrm{H}(7 \mathrm{~A})$ & -4159 & 3016 & 7617 & 54 \\
\hline $\mathrm{H}(7 \mathrm{~B})$ & -4436 & 2911 & 6212 & 54 \\
\hline $\mathrm{H}(9)$ & -4236 & 1775 & 7704 & 51 \\
\hline $\mathrm{H}(11)$ & -999 & 619 & 7389 & 52 \\
\hline $\mathrm{H}(12)$ & -25 & 1618 & 6727 & 48 \\
\hline $\mathrm{H}(16 \mathrm{~A})$ & 2012 & 2523 & 7595 & 53 \\
\hline $\mathrm{H}(16 \mathrm{~B})$ & 789 & 2494 & 8280 & 53 \\
\hline $\mathrm{H}(17 \mathrm{~A})$ & 1284 & 3609 & 9060 & 55 \\
\hline $\mathrm{H}(17 \mathrm{~B})$ & 2624 & 3150 & 9354 & 55 \\
\hline $\mathrm{H}(18 \mathrm{~A})$ & 2258 & 4559 & 6724 & 61 \\
\hline $\mathrm{H}(18 \mathrm{~B})$ & 1061 & 4487 & 7436 & 61 \\
\hline $\mathrm{H}(19 \mathrm{~A})$ & 1750 & 3486 & 5808 & 58 \\
\hline $\mathrm{H}(19 \mathrm{~B})$ & 397 & 3932 & 5619 & 58 \\
\hline $\mathrm{H}(22)$ & 6671 & 2797 & 9861 & 56 \\
\hline $\mathrm{H}(23)$ & 8941 & 2988 & 10497 & 60 \\
\hline $\mathrm{H}(24)$ & 9808 & 4124 & 10634 & 61 \\
\hline $\mathrm{H}(25)$ & 8354 & 5072 & 10227 & 58 \\
\hline $\mathrm{H}(26)$ & 6073 & 4896 & 9585 & 55 \\
\hline $\mathrm{H}(3)$ & $4520(40)$ & $3280(20)$ & $9250(40)$ & $33(12)$ \\
\hline
\end{tabular}


Table S11. Torsion angles $\left[^{\circ}\right]$ for $( \pm)-6 b$.

\begin{tabular}{|c|c|}
\hline $\mathrm{O}(1)-\mathrm{N}(1)-\mathrm{C}(1)-\mathrm{C}(5)$ & $-173.6(4)$ \\
\hline$C(2)-N(1)-C(1)-C(5)$ & $5.8(6)$ \\
\hline $\mathrm{O}(1)-\mathrm{N}(1)-\mathrm{C}(1)-\mathrm{C}(14)$ & $7.9(6)$ \\
\hline $\mathrm{C}(2)-\mathrm{N}(1)-\mathrm{C}(1)-\mathrm{C}(14)$ & $-172.8(4)$ \\
\hline $\mathrm{O}(1)-\mathrm{N}(1)-\mathrm{C}(2)-\mathrm{C}(3)$ & $175.5(4)$ \\
\hline$C(1)-N(1)-C(2)-C(3)$ & $-3.9(6)$ \\
\hline $\mathrm{N}(1)-\mathrm{C}(2)-\mathrm{C}(3)-\mathrm{C}(4)$ & $-0.4(7)$ \\
\hline$C(2)-C(3)-C(4)-C(5)$ & $2.7(7)$ \\
\hline$C(3)-C(4)-C(5)-C(1)$ & $-0.7(6)$ \\
\hline$C(3)-C(4)-C(5)-C(6)$ & $-176.3(4)$ \\
\hline $\mathrm{N}(1)-\mathrm{C}(1)-\mathrm{C}(5)-\mathrm{C}(4)$ & $-3.5(6)$ \\
\hline$C(14)-C(1)-C(5)-C(4)$ & $175.0(4)$ \\
\hline $\mathrm{N}(1)-\mathrm{C}(1)-\mathrm{C}(5)-\mathrm{C}(6)$ & $172.4(4)$ \\
\hline$C(14)-C(1)-C(5)-C(6)$ & $-9.1(6)$ \\
\hline$C(4)-C(5)-C(6)-C(7)$ & $107.7(5)$ \\
\hline$C(1)-C(5)-C(6)-C(7)$ & $-68.1(5)$ \\
\hline$C(5)-C(6)-C(7)-C(8)$ & $71.7(5)$ \\
\hline$C(6)-C(7)-C(8)-C(9)$ & $164.2(4)$ \\
\hline$C(6)-C(7)-C(8)-C(13)$ & $-16.8(6)$ \\
\hline $\mathrm{C}(13)-\mathrm{C}(8)-\mathrm{C}(9)-\mathrm{C}(10)$ & $-2.0(6)$ \\
\hline $\mathrm{C}(7)-\mathrm{C}(8)-\mathrm{C}(9)-\mathrm{C}(10)$ & $177.1(4)$ \\
\hline$C(8)-C(9)-C(10)-C(11)$ & $-1.3(7)$ \\
\hline $\mathrm{C}(8)-\mathrm{C}(9)-\mathrm{C}(10)-\mathrm{Cl}(1)$ & $179.5(3)$ \\
\hline$C(9)-C(10)-C(11)-C(12)$ & $2.7(7)$ \\
\hline $\mathrm{Cl}(1)-\mathrm{C}(10)-\mathrm{C}(11)-\mathrm{C}(12)$ & $-178.1(3)$ \\
\hline $\mathrm{C}(10)-\mathrm{C}(11)-\mathrm{C}(12)-\mathrm{C}(13)$ & $-0.8(7)$ \\
\hline$C(11)-C(12)-C(13)-C(8)$ & $-2.3(6)$ \\
\hline$C(11)-C(12)-C(13)-C(14)$ & $-178.8(4)$ \\
\hline$C(9)-C(8)-C(13)-C(12)$ & $3.7(6)$ \\
\hline$C(7)-C(8)-C(13)-C(12)$ & $-175.3(4)$ \\
\hline$C(9)-C(8)-C(13)-C(14)$ & $-180.0(4)$ \\
\hline$C(7)-C(8)-C(13)-C(14)$ & $1.0(7)$ \\
\hline $\mathrm{N}(1)-\mathrm{C}(1)-\mathrm{C}(14)-\mathrm{C}(15)$ & $72.0(5)$ \\
\hline$C(5)-C(1)-C(14)-C(15)$ & $-106.6(5)$ \\
\hline $\mathrm{N}(1)-\mathrm{C}(1)-\mathrm{C}(14)-\mathrm{C}(13)$ & $-109.7(4)$ \\
\hline
\end{tabular}




\begin{tabular}{|c|c|}
\hline$C(5)-C(1)-C(14)-C(13)$ & $71.8(5)$ \\
\hline$C(12)-C(13)-C(14)-C(15)$ & $-51.4(6)$ \\
\hline$C(8)-C(13)-C(14)-C(15)$ & $132.2(5)$ \\
\hline $\mathrm{C}(12)-\mathrm{C}(13)-\mathrm{C}(14)-\mathrm{C}(1)$ & $130.4(4)$ \\
\hline $\mathrm{C}(8)-\mathrm{C}(13)-\mathrm{C}(14)-\mathrm{C}(1)$ & $-46.0(5)$ \\
\hline$C(1)-C(14)-C(15)-C(16)$ & $172.9(4)$ \\
\hline$C(13)-C(14)-C(15)-C(16)$ & $-5.3(7)$ \\
\hline$C(1)-C(14)-C(15)-C(19)$ & $-7.0(6)$ \\
\hline$C(13)-C(14)-C(15)-C(19)$ & $174.8(4)$ \\
\hline $\mathrm{C}(14)-\mathrm{C}(15)-\mathrm{C}(16)-\mathrm{C}(17)$ & $-131.2(4)$ \\
\hline$C(19)-C(15)-C(16)-C(17)$ & $48.8(5)$ \\
\hline $\mathrm{C}(20)-\mathrm{N}(2)-\mathrm{C}(17)-\mathrm{C}(16)$ & $-124.5(5)$ \\
\hline $\mathrm{C}(18)-\mathrm{N}(2)-\mathrm{C}(17)-\mathrm{C}(16)$ & $59.1(5)$ \\
\hline $\mathrm{C}(15)-\mathrm{C}(16)-\mathrm{C}(17)-\mathrm{N}(2)$ & $-53.6(5)$ \\
\hline $\mathrm{C}(20)-\mathrm{N}(2)-\mathrm{C}(18)-\mathrm{C}(19)$ & $124.6(4)$ \\
\hline $\mathrm{C}(17)-\mathrm{N}(2)-\mathrm{C}(18)-\mathrm{C}(19)$ & $-58.6(5)$ \\
\hline $\mathrm{N}(2)-\mathrm{C}(18)-\mathrm{C}(19)-\mathrm{C}(15)$ & $52.5(5)$ \\
\hline$C(14)-C(15)-C(19)-C(18)$ & $131.4(4)$ \\
\hline$C(16)-C(15)-C(19)-C(18)$ & $-48.5(5)$ \\
\hline $\mathrm{C}(17)-\mathrm{N}(2)-\mathrm{C}(20)-\mathrm{O}(2)$ & $-171.1(4)$ \\
\hline $\mathrm{C}(18)-\mathrm{N}(2)-\mathrm{C}(20)-\mathrm{O}(2)$ & $5.1(6)$ \\
\hline $\mathrm{C}(17)-\mathrm{N}(2)-\mathrm{C}(20)-\mathrm{N}(3)$ & $8.9(6)$ \\
\hline $\mathrm{C}(18)-\mathrm{N}(2)-\mathrm{C}(20)-\mathrm{N}(3)$ & $-174.9(4)$ \\
\hline $\mathrm{C}(21)-\mathrm{N}(3)-\mathrm{C}(20)-\mathrm{O}(2)$ & $0.6(6)$ \\
\hline $\mathrm{C}(21)-\mathrm{N}(3)-\mathrm{C}(20)-\mathrm{N}(2)$ & $-179.4(4)$ \\
\hline $\mathrm{C}(20)-\mathrm{N}(3)-\mathrm{C}(21)-\mathrm{C}(22)$ & $-143.8(4)$ \\
\hline $\mathrm{C}(20)-\mathrm{N}(3)-\mathrm{C}(21)-\mathrm{C}(26)$ & $37.6(6)$ \\
\hline$C(26)-C(21)-C(22)-C(23)$ & $-2.0(6)$ \\
\hline $\mathrm{N}(3)-\mathrm{C}(21)-\mathrm{C}(22)-\mathrm{C}(23)$ & $179.3(4)$ \\
\hline$C(21)-C(22)-C(23)-C(24)$ & $0.1(7)$ \\
\hline$C(22)-C(23)-C(24)-C(25)$ & $1.9(7)$ \\
\hline$C(23)-C(24)-C(25)-C(26)$ & $-1.9(7)$ \\
\hline$C(24)-C(25)-C(26)-C(21)$ & $0.0(7)$ \\
\hline$C(22)-C(21)-C(26)-C(25)$ & $2.0(6)$ \\
\hline $\mathrm{N}(3)-\mathrm{C}(21)-\mathrm{C}(26)-\mathrm{C}(25)$ & $-179.4(4)$ \\
\hline
\end{tabular}


Table S12. Hydrogen bonds for $( \pm)-6 \mathbf{b}\left[\AA ̊\right.$ and $\left.{ }^{\circ}\right]$.

\begin{tabular}{lllll}
\hline $\mathrm{D}-\mathrm{H} . . . \mathrm{A}$ & $\mathrm{d}(\mathrm{D}-\mathrm{H})$ & $\mathrm{d}(\mathrm{H} \ldots \mathrm{A})$ & $\mathrm{d}(\mathrm{D} \ldots \mathrm{A})$ & $<(\mathrm{DHA})$ \\
\hline $\mathrm{N}(3)-\mathrm{H}(3) \ldots \mathrm{O}(1) \# 1$ & $0.83(4)$ & $2.09(5)$ & $2.881(5)$ & $160(4)$ \\
\hline
\end{tabular}

Symmetry transformations used to generate equivalent atoms: $\# 1 \mathrm{x}+1 / 2,-\mathrm{y}+1 / 2, \mathrm{z}+1 / 2$

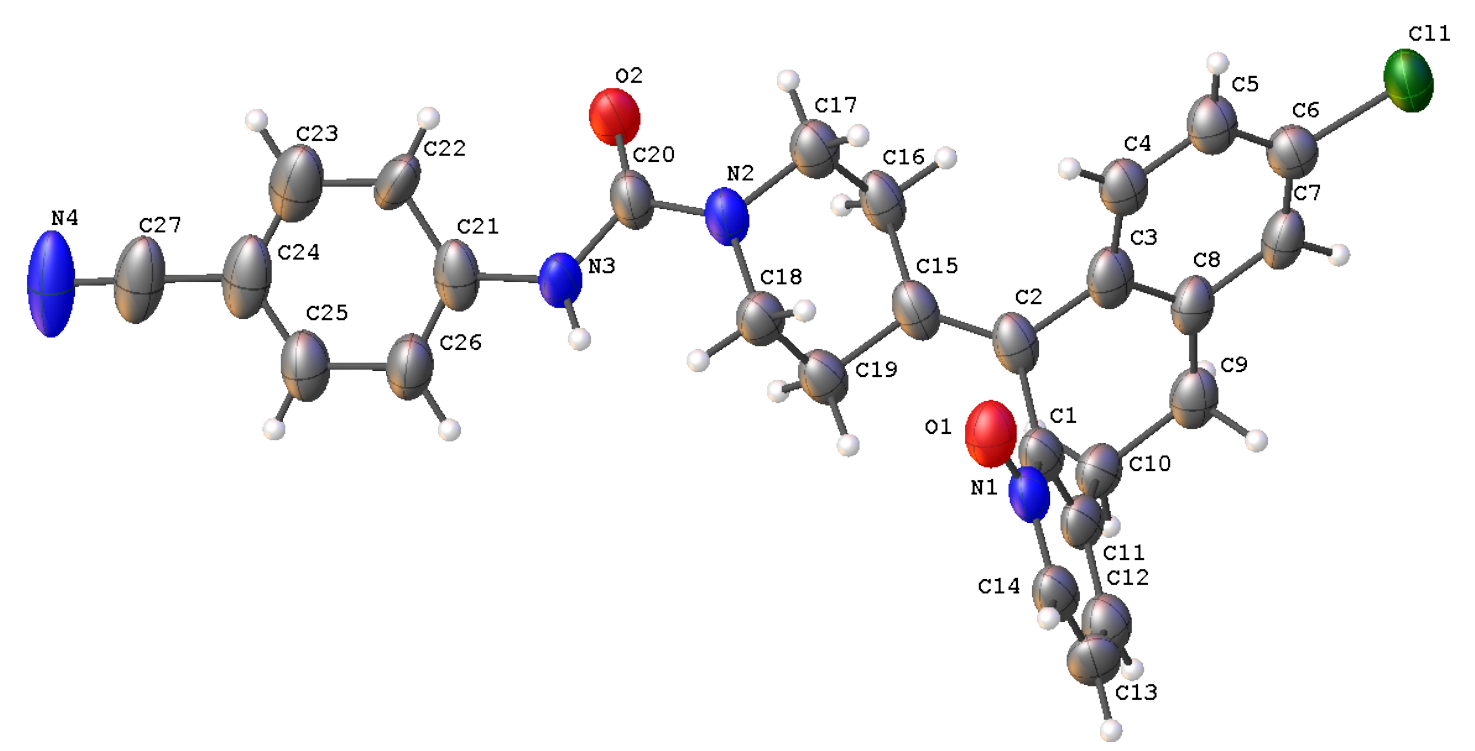

Figure S7. The complete numbering scheme of (-)-6f with 50\% thermal ellipsoid probability levels. The hydrogen atoms are shown as circles for clarity. 
Table S13. Crystal data and structure refinement for (-)-6f.

Identification code

Empirical formula

Formula weight

Temperature

Wavelength

Crystal system

Space group

Unit cell dimensions

Volume

Z

Density (calculated)

Absorption coefficient

$\mathrm{F}(000)$

Crystal size

Crystal color and habit

Diffractometer

Theta range for data collection

Index ranges

Reflections collected

Independent reflections

Observed reflections $(\mathrm{I}>2 \operatorname{sigma}(\mathrm{I}))$

Completeness to theta $=62.354^{\circ}$

Absorption correction

Max. and min. transmission

Solution method

Refinement method

Data / restraints / parameters

Goodness-of-fit on $\mathrm{F}^{2}$

Final R indices $[\mathrm{I}>2 \operatorname{sigma}(\mathrm{I})]$

$\mathrm{R}$ indices (all data)

Absolute structure parameter

Largest diff. peak and hole
1983464

$\mathrm{C}_{27} \mathrm{H}_{23} \mathrm{ClN}_{4} \mathrm{O}_{2}$

470.94

93(2) K

$1.54184 \AA$

Monoclinic

Pc

$\mathrm{a}=5.0825(3) \AA \quad \alpha=90^{\circ}$

$\mathrm{b}=20.6949(12) \AA$

$\beta=93.363(6)^{\circ}$

$\mathrm{c}=11.3564(8) \AA$

$\gamma=90^{\circ}$

$1192.43(13) \AA^{3}$

2

$1.312 \mathrm{Mg} / \mathrm{m}^{3}$

$1.674 \mathrm{~mm}^{-1}$

492

$0.400 \times 0.020 \times 0.020 \mathrm{~mm}^{3}$

Colorless Plate

Rigaku Saturn 944+ CCD

2.135 to $62.354^{\circ}$

$-5<=\mathrm{h}<=5,-23<=\mathrm{k}<=23,-13<=\mathrm{l}<=13$

36602

$3683[\mathrm{R}(\mathrm{int})=0.2319]$

2698

$99.9 \%$

Semi-empirical from equivalents

1.00000 and 0.49354

SHELXT-2014/5 (Sheldrick, 2014)

SHELXL-2014/7 (Sheldrick, 2014)

$3683 / 2 / 311$

1.069

$\mathrm{R} 1=0.0828, \mathrm{wR} 2=0.2131$

$\mathrm{R} 1=0.1124, \mathrm{wR} 2=0.2338$

$-0.01(3)$

0.317 and -0.539 e. $\AA^{-3}$ 
Table S14. Atomic coordinates $\left(\mathrm{x} 10^{4}\right)$ and equivalent isotropic displacement parameters $\left(\AA^{2} \times 10^{3}\right)$ for $(-)-6 f$. $\mathrm{U}(\mathrm{eq})$ is defined as one third of the trace of the orthogonalized $\mathrm{U}^{\mathrm{ij}}$ tensor.

\begin{tabular}{|c|c|c|c|c|}
\hline & $\mathrm{x}$ & $\mathrm{y}$ & $\mathrm{z}$ & $\mathrm{U}(\mathrm{eq})$ \\
\hline $\mathrm{Cl}(1)$ & $13355(5)$ & $3882(1)$ & $6594(3)$ & $60(1)$ \\
\hline $\mathrm{O}(1)$ & $12956(15)$ & $7515(4)$ & $7124(8)$ & $62(2)$ \\
\hline $\mathrm{O}(2)$ & $5368(14)$ & $7637(3)$ & $1951(7)$ & $59(2)$ \\
\hline $\mathrm{N}(1)$ & $11421(18)$ & $7341(4)$ & $7972(8)$ & $52(2)$ \\
\hline $\mathrm{N}(2)$ & $8370(17)$ & $7705(4)$ & $3523(8)$ & $54(2)$ \\
\hline $\mathrm{N}(3)$ & $5315(18)$ & $8538(4)$ & $3100(9)$ & $55(2)$ \\
\hline $\mathrm{N}(4)$ & $-3280(20)$ & $10477(5)$ & $31(14)$ & $106(5)$ \\
\hline$C(1)$ & $9480(20)$ & $6905(5)$ & $7738(10)$ & $51(3)$ \\
\hline$C(2)$ & $9079(17)$ & $6598(5)$ & $6552(10)$ & $50(3)$ \\
\hline$C(3)$ & $9888(19)$ & $5909(5)$ & $6545(10)$ & $54(3)$ \\
\hline$C(4)$ & $11782(19)$ & $5720(5)$ & $5749(10)$ & $49(2)$ \\
\hline$C(5)$ & $12805(19)$ & $5102(5)$ & $5727(10)$ & $53(3)$ \\
\hline$C(6)$ & $12020(20)$ & $4655(5)$ & $6524(10)$ & $50(2)$ \\
\hline$C(7)$ & $10150(20)$ & $4811(5)$ & $7310(9)$ & $51(3)$ \\
\hline$C(8)$ & $9037(18)$ & $5443(5)$ & $7360(9)$ & $48(2)$ \\
\hline$C(9)$ & $7090(20)$ & $5551(5)$ & $8274(10)$ & $53(3)$ \\
\hline$C(10)$ & $5835(19)$ & $6217(5)$ & $8356(9)$ & $49(2)$ \\
\hline$C(11)$ & $7847(18)$ & $6730(5)$ & $8662(10)$ & $49(2)$ \\
\hline$C(12)$ & $8250(20)$ & $7009(5)$ & $9747(11)$ & $55(3)$ \\
\hline$C(13)$ & $10260(20)$ & $7444(5)$ & $9944(10)$ & $55(3)$ \\
\hline $\mathrm{C}(14)$ & $11850(20)$ & $7601(5)$ & $9044(10)$ & $55(3)$ \\
\hline$C(15)$ & $8110(20)$ & $6935(4)$ & $5621(11)$ & $53(3)$ \\
\hline$C(16)$ & $7462(18)$ & $6655(4)$ & $4423(10)$ & $51(3)$ \\
\hline$C(17)$ & $8900(20)$ & $7015(5)$ & $3460(11)$ & $54(3)$ \\
\hline$C(18)$ & $9120(20)$ & $7970(5)$ & $4659(9)$ & $51(3)$ \\
\hline$C(19)$ & $7640(20)$ & $7655(4)$ & $5646(10)$ & $53(3)$ \\
\hline$C(20)$ & $6240(20)$ & 7939(4) & $2801(10)$ & $55(3)$ \\
\hline$C(21)$ & $3510(20)$ & $8907(5)$ & $2422(12)$ & $61(3)$ \\
\hline$C(22)$ & $3300(30)$ & $8880(5)$ & $1182(10)$ & $65(3)$ \\
\hline$C(23)$ & $1560(30)$ & $9264(6)$ & $582(15)$ & $88(5)$ \\
\hline$C(24)$ & $-110(30)$ & $9695(6)$ & $1162(14)$ & $78(4)$ \\
\hline$C(25)$ & $80(20)$ & $9709(6)$ & $2358(13)$ & $67(3)$ \\
\hline
\end{tabular}




\begin{tabular}{lrrrr}
$\mathrm{C}(26)$ & $1860(20)$ & $9319(5)$ & $3002(12)$ & $65(3)$ \\
$\mathrm{C}(27)$ & $-1890(30)$ & $10116(6)$ & $534(14)$ & $88(5)$ \\
\hline
\end{tabular}

Table S15. Bond lengths $[\AA]$ and angles $\left[{ }^{\circ}\right]$ for $(-)-6 f$.

$\begin{array}{ll}\mathrm{Cl}(1)-\mathrm{C}(6) & 1.737(10) \\ \mathrm{O}(1)-\mathrm{N}(1) & 1.324(13) \\ \mathrm{O}(2)-\mathrm{C}(20) & 1.211(12) \\ \mathrm{N}(1)-\mathrm{C}(14) & 1.338(14) \\ \mathrm{N}(1)-\mathrm{C}(1) & 1.352(14) \\ \mathrm{N}(2)-\mathrm{C}(20) & 1.403(13) \\ \mathrm{N}(2)-\mathrm{C}(18) & 1.432(13) \\ \mathrm{N}(2)-\mathrm{C}(17) & 1.455(12) \\ \mathrm{N}(3)-\mathrm{C}(20) & 1.376(13) \\ \mathrm{N}(3)-\mathrm{C}(21) & 1.390(13) \\ \mathrm{N}(3)-\mathrm{H}(3) & 0.81(11) \\ \mathrm{N}(4)-\mathrm{C}(27) & 1.154(16) \\ \mathrm{C}(1)-\mathrm{C}(11) & 1.422(16) \\ \mathrm{C}(1)-\mathrm{C}(2) & 1.492(16) \\ \mathrm{C}(2)-\mathrm{C}(15) & 1.336(15) \\ \mathrm{C}(2)-\mathrm{C}(3) & 1.485(14) \\ \mathrm{C}(3)-\mathrm{C}(4) & 1.414(16) \\ \mathrm{C}(3)-\mathrm{C}(8) & 1.421(15) \\ \mathrm{C}(4)-\mathrm{C}(5) & 1.380(14) \\ \mathrm{C}(4)-\mathrm{H}(4) & 0.9500 \\ \mathrm{C}(5)-\mathrm{C}(6) & 1.372(15) \\ \mathrm{C}(5)-\mathrm{H}(5) & 0.9500 \\ \mathrm{C}(6)-\mathrm{C}(7) & 1.378(16) \\ \mathrm{C}(7)-\mathrm{C}(8) & 1.428(14) \\ \mathrm{C}(7)-\mathrm{H}(7) & 0.9500 \\ \mathrm{C}(8)-\mathrm{C}(9) & 1.491(16) \\ \mathrm{C}(9)-\mathrm{C}(10) & 1.524(14) \\ \mathrm{C}(9)-\mathrm{H}(9 \mathrm{~A}) & 0.9900 \\ \mathrm{C}(9)-\mathrm{H}(9 \mathrm{~B}) & 0.9900 \\ \mathrm{C}(10)-\mathrm{C}(11) & 1.500(14) \\ \mathrm{C}(10)-\mathrm{H}(10 \mathrm{~A}) & \end{array}$




\begin{tabular}{|c|c|}
\hline $\mathrm{C}(10)-\mathrm{H}(10 \mathrm{~B})$ & 0.9900 \\
\hline$C(11)-C(12)$ & $1.365(15)$ \\
\hline$C(12)-C(13)$ & $1.369(15)$ \\
\hline $\mathrm{C}(12)-\mathrm{H}(12)$ & 0.9500 \\
\hline$C(13)-C(14)$ & $1.379(17)$ \\
\hline $\mathrm{C}(13)-\mathrm{H}(13)$ & 0.9500 \\
\hline $\mathrm{C}(14)-\mathrm{H}(14)$ & 0.9500 \\
\hline$C(15)-C(16)$ & $1.497(16)$ \\
\hline$C(15)-C(19)$ & $1.511(12)$ \\
\hline$C(16)-C(17)$ & $1.543(17)$ \\
\hline $\mathrm{C}(16)-\mathrm{H}(16 \mathrm{~A})$ & 0.9900 \\
\hline$C(16)-H(16 B)$ & 0.9900 \\
\hline $\mathrm{C}(17)-\mathrm{H}(17 \mathrm{~A})$ & 0.9900 \\
\hline $\mathrm{C}(17)-\mathrm{H}(17 \mathrm{~B})$ & 0.9900 \\
\hline$C(18)-C(19)$ & $1.532(16)$ \\
\hline $\mathrm{C}(18)-\mathrm{H}(18 \mathrm{~A})$ & 0.9900 \\
\hline $\mathrm{C}(18)-\mathrm{H}(18 \mathrm{~B})$ & 0.9900 \\
\hline $\mathrm{C}(19)-\mathrm{H}(19 \mathrm{~A})$ & 0.9900 \\
\hline $\mathrm{C}(19)-\mathrm{H}(19 \mathrm{~B})$ & 0.9900 \\
\hline$C(21)-C(26)$ & $1.392(19)$ \\
\hline$C(21)-C(22)$ & $1.407(18)$ \\
\hline$C(22)-C(23)$ & $1.345(19)$ \\
\hline $\mathrm{C}(22)-\mathrm{H}(22)$ & 0.9500 \\
\hline$C(23)-C(24)$ & $1.42(2)$ \\
\hline $\mathrm{C}(23)-\mathrm{H}(23)$ & 0.9500 \\
\hline$C(24)-C(25)$ & $1.36(2)$ \\
\hline$C(24)-C(27)$ & $1.418(18)$ \\
\hline$C(25)-C(26)$ & $1.387(16)$ \\
\hline $\mathrm{C}(25)-\mathrm{H}(25)$ & 0.9500 \\
\hline $\mathrm{C}(26)-\mathrm{H}(26)$ & 0.9500 \\
\hline $\mathrm{O}(1)-\mathrm{N}(1)-\mathrm{C}(14)$ & $118.7(9)$ \\
\hline $\mathrm{O}(1)-\mathrm{N}(1)-\mathrm{C}(1)$ & 119.6(9) \\
\hline $\mathrm{C}(14)-\mathrm{N}(1)-\mathrm{C}(1)$ & $121.7(11)$ \\
\hline $\mathrm{C}(20)-\mathrm{N}(2)-\mathrm{C}(18)$ & $123.3(9)$ \\
\hline$C(20)-N(2)-C(17)$ & $116.7(8)$ \\
\hline
\end{tabular}




\begin{tabular}{|c|c|}
\hline $\mathrm{C}(18)-\mathrm{N}(2)-\mathrm{C}(17)$ & $112.3(8)$ \\
\hline $\mathrm{C}(20)-\mathrm{N}(3)-\mathrm{C}(21)$ & $125.6(10)$ \\
\hline $\mathrm{C}(20)-\mathrm{N}(3)-\mathrm{H}(3)$ & $120(7)$ \\
\hline $\mathrm{C}(21)-\mathrm{N}(3)-\mathrm{H}(3)$ & $113(7)$ \\
\hline $\mathrm{N}(1)-\mathrm{C}(1)-\mathrm{C}(11)$ & $118.4(10)$ \\
\hline $\mathrm{N}(1)-\mathrm{C}(1)-\mathrm{C}(2)$ & $121.3(10)$ \\
\hline$C(11)-C(1)-C(2)$ & $120.2(9)$ \\
\hline$C(15)-C(2)-C(3)$ & $125.9(10)$ \\
\hline$C(15)-C(2)-C(1)$ & 121.1(9) \\
\hline$C(3)-C(2)-C(1)$ & $113.0(9)$ \\
\hline$C(4)-C(3)-C(8)$ & $118.3(9)$ \\
\hline$C(4)-C(3)-C(2)$ & $117.9(10)$ \\
\hline$C(8)-C(3)-C(2)$ & $123.6(10)$ \\
\hline$C(5)-C(4)-C(3)$ & $122.7(10)$ \\
\hline $\mathrm{C}(5)-\mathrm{C}(4)-\mathrm{H}(4)$ & 118.7 \\
\hline $\mathrm{C}(3)-\mathrm{C}(4)-\mathrm{H}(4)$ & 118.7 \\
\hline$C(6)-C(5)-C(4)$ & $119.2(11)$ \\
\hline $\mathrm{C}(6)-\mathrm{C}(5)-\mathrm{H}(5)$ & 120.4 \\
\hline $\mathrm{C}(4)-\mathrm{C}(5)-\mathrm{H}(5)$ & 120.4 \\
\hline$C(5)-C(6)-C(7)$ & $120.5(9)$ \\
\hline$C(5)-C(6)-C l(1)$ & $121.6(9)$ \\
\hline $\mathrm{C}(7)-\mathrm{C}(6)-\mathrm{Cl}(1)$ & $117.9(8)$ \\
\hline$C(6)-C(7)-C(8)$ & $122.1(9)$ \\
\hline $\mathrm{C}(6)-\mathrm{C}(7)-\mathrm{H}(7)$ & 119.0 \\
\hline $\mathrm{C}(8)-\mathrm{C}(7)-\mathrm{H}(7)$ & 119.0 \\
\hline$C(3)-C(8)-C(7)$ & $117.3(10)$ \\
\hline$C(3)-C(8)-C(9)$ & $126.2(9)$ \\
\hline$C(7)-C(8)-C(9)$ & $116.5(9)$ \\
\hline$C(8)-C(9)-C(10)$ & $118.5(9)$ \\
\hline $\mathrm{C}(8)-\mathrm{C}(9)-\mathrm{H}(9 \mathrm{~A})$ & 107.7 \\
\hline $\mathrm{C}(10)-\mathrm{C}(9)-\mathrm{H}(9 \mathrm{~A})$ & 107.7 \\
\hline $\mathrm{C}(8)-\mathrm{C}(9)-\mathrm{H}(9 \mathrm{~B})$ & 107.7 \\
\hline $\mathrm{C}(10)-\mathrm{C}(9)-\mathrm{H}(9 \mathrm{~B})$ & 107.7 \\
\hline $\mathrm{H}(9 \mathrm{~A})-\mathrm{C}(9)-\mathrm{H}(9 \mathrm{~B})$ & 107.1 \\
\hline$C(11)-C(10)-C(9)$ & $111.8(8)$ \\
\hline $\mathrm{C}(11)-\mathrm{C}(10)-\mathrm{H}(10 \mathrm{~A})$ & 109.3 \\
\hline
\end{tabular}




\begin{tabular}{ll}
$\mathrm{C}(9)-\mathrm{C}(10)-\mathrm{H}(10 \mathrm{~A})$ & 109.3 \\
$\mathrm{C}(11)-\mathrm{C}(10)-\mathrm{H}(10 \mathrm{~B})$ & 109.3 \\
$\mathrm{C}(9)-\mathrm{C}(10)-\mathrm{H}(10 \mathrm{~B})$ & 109.3 \\
$\mathrm{H}(10 \mathrm{~A})-\mathrm{C}(10)-\mathrm{H}(10 \mathrm{~B})$ & 107.9 \\
$\mathrm{C}(12)-\mathrm{C}(11)-\mathrm{C}(1)$ & $119.6(10)$ \\
$\mathrm{C}(12)-\mathrm{C}(11)-\mathrm{C}(10)$ & $124.9(11)$ \\
$\mathrm{C}(1)-\mathrm{C}(11)-\mathrm{C}(10)$ & $115.4(9)$ \\
$\mathrm{C}(11)-\mathrm{C}(12)-\mathrm{C}(13)$ & $119.8(12)$ \\
$\mathrm{C}(11)-\mathrm{C}(12)-\mathrm{H}(12)$ & 120.1 \\
$\mathrm{C}(13)-\mathrm{C}(12)-\mathrm{H}(12)$ & 120.1 \\
$\mathrm{C}(12)-\mathrm{C}(13)-\mathrm{C}(14)$ & $119.8(10)$ \\
$\mathrm{C}(12)-\mathrm{C}(13)-\mathrm{H}(13)$ & 120.1 \\
$\mathrm{C}(14)-\mathrm{C}(13)-\mathrm{H}(13)$ & 120.1 \\
$\mathrm{~N}(1)-\mathrm{C}(14)-\mathrm{C}(13)$ & $120.5(10)$ \\
$\mathrm{N}(1)-\mathrm{C}(14)-\mathrm{H}(14)$ & 119.7 \\
$\mathrm{C}(13)-\mathrm{C}(14)-\mathrm{H}(14)$ & 119.7 \\
$\mathrm{C}(2)-\mathrm{C}(15)-\mathrm{C}(16)$ & $124.6(9)$ \\
$\mathrm{C}(2)-\mathrm{C}(15)-\mathrm{C}(19)$ & $123.5(10)$ \\
$\mathrm{C}(16)-\mathrm{C}(15)-\mathrm{C}(19)$ & $111.8(9)$ \\
$\mathrm{C}(15)-\mathrm{C}(16)-\mathrm{C}(17)$ & $111.7(8)$ \\
$\mathrm{C}(15)-\mathrm{C}(16)-\mathrm{H}(16 \mathrm{~A})$ & 109.3 \\
$\mathrm{C}(17)-\mathrm{C}(16)-\mathrm{H}(16 \mathrm{~A})$ & 109.3 \\
$\mathrm{C}(15)-\mathrm{C}(16)-\mathrm{H}(16 \mathrm{~B})$ & 109.3 \\
$\mathrm{C}(17)-\mathrm{C}(16)-\mathrm{H}(16 \mathrm{~B})$ & 109.3 \\
$\mathrm{H}(16 \mathrm{~A})-\mathrm{C}(16)-\mathrm{H}(16 \mathrm{~B})$ & 107.9 \\
$\mathrm{~N}(2)-\mathrm{C}(17)-\mathrm{C}(16)$ & $109.9(9)$ \\
$\mathrm{N}(2)-\mathrm{C}(17)-\mathrm{H}(17 \mathrm{~A})$ & 109.7 \\
$\mathrm{C}(16)-\mathrm{C}(17)-\mathrm{H}(17 \mathrm{~A})$ & 109.7 \\
$\mathrm{~N}(2)-\mathrm{C}(17)-\mathrm{H}(17 \mathrm{~B})$ & 109.7 \\
$\mathrm{C}(16)-\mathrm{C}(17)-\mathrm{H}(17 \mathrm{~B})$ & 109.7 \\
$\mathrm{H}(17 \mathrm{~A})-\mathrm{C}(17)-\mathrm{H}(17 \mathrm{~B})$ & 108.2 \\
$\mathrm{~N}(2)-\mathrm{C}(18)-\mathrm{C}(19)$ & $112.4(8)$ \\
$\mathrm{N}(19)-\mathrm{C}(18)-\mathrm{H}(18 \mathrm{~A})$ & 109.1 \\
& 109.1 \\
$\mathrm{C}(18 \mathrm{~A}) \mathrm{H}(18 \mathrm{~B})$ & 109.1 \\
& 109.1 \\
\hline
\end{tabular}




$\begin{array}{ll}\mathrm{H}(18 \mathrm{~A})-\mathrm{C}(18)-\mathrm{H}(18 \mathrm{~B}) & 107.9 \\ \mathrm{C}(15)-\mathrm{C}(19)-\mathrm{C}(18) & 108.6(9) \\ \mathrm{C}(15)-\mathrm{C}(19)-\mathrm{H}(19 \mathrm{~A}) & 110.0 \\ \mathrm{C}(18)-\mathrm{C}(19)-\mathrm{H}(19 \mathrm{~A}) & 110.0 \\ \mathrm{C}(15)-\mathrm{C}(19)-\mathrm{H}(19 \mathrm{~B}) & 110.0 \\ \mathrm{C}(18)-\mathrm{C}(19)-\mathrm{H}(19 \mathrm{~B}) & 110.0 \\ \mathrm{H}(19 \mathrm{~A})-\mathrm{C}(19)-\mathrm{H}(19 \mathrm{~B}) & 108.4 \\ \mathrm{O}(2)-\mathrm{C}(20)-\mathrm{N}(3) & 123.0(9) \\ \mathrm{O}(2)-\mathrm{C}(20)-\mathrm{N}(2) & 121.5(8) \\ \mathrm{N}(3)-\mathrm{C}(20)-\mathrm{N}(2) & 115.5(9) \\ \mathrm{N}(3)-\mathrm{C}(21)-\mathrm{C}(26) & 118.1(12) \\ \mathrm{N}(3)-\mathrm{C}(21)-\mathrm{C}(22) & 122.9(12) \\ \mathrm{C}(26)-\mathrm{C}(21)-\mathrm{C}(22) & 119.0(10) \\ \mathrm{C}(23)-\mathrm{C}(22)-\mathrm{C}(21) & 119.6(14) \\ \mathrm{C}(23)-\mathrm{C}(22)-\mathrm{H}(22) & 120.2 \\ \mathrm{C}(21)-\mathrm{C}(22)-\mathrm{H}(22) & 120.2 \\ \mathrm{C}(22)-\mathrm{C}(23)-\mathrm{C}(24) & 122.0(14) \\ \mathrm{C}(22)-\mathrm{C}(23)-\mathrm{H}(23) & 119.0 \\ \mathrm{C}(24)-\mathrm{C}(23)-\mathrm{H}(23) & 119.0 \\ \mathrm{C}(25)-\mathrm{C}(24)-\mathrm{C}(27) & 119.9(16) \\ \mathrm{C}(25)-\mathrm{C}(24)-\mathrm{C}(23) & 117.8(11) \\ \mathrm{C}(27)-\mathrm{C}(24)-\mathrm{C}(23) & 122.2(14) \\ \mathrm{C}(24)-\mathrm{C}(25)-\mathrm{C}(26) & 121.5(14) \\ \mathrm{C}(24)-\mathrm{C}(25)-\mathrm{H}(25) & 119.2 \\ \mathrm{C}(26)-\mathrm{C}(25)-\mathrm{H}(25) & 119.2 \\ \mathrm{C}(25)-\mathrm{C}(26)-\mathrm{C}(21) & 120.0(13) \\ \mathrm{C}(25)-\mathrm{C}(26)-\mathrm{H}(26) & 120.0 \\ \mathrm{C}(21)-\mathrm{C}(26)-\mathrm{H}(26) & 120.0 \\ \mathrm{~N}(4)-\mathrm{C}(27)-\mathrm{C}(24) & 177.4(12) \\ & \end{array}$


Table S16. Anisotropic displacement parameters $\left(\AA^{2} \times 10^{3}\right)$ for (-)-6f. The anisotropic displacement factor exponent takes the form: $-2 \pi^{2}\left[h^{2} a^{* 2} U^{11}+\ldots+2 h k a^{*} b^{*} U^{12}\right]$

\begin{tabular}{|c|c|c|c|c|c|c|}
\hline & $\mathrm{U}^{11}$ & $\mathrm{U}^{22}$ & $\mathrm{U}^{33}$ & $\mathrm{U}^{23}$ & $\mathrm{U}^{13}$ & $\mathrm{U}^{12}$ \\
\hline $\mathrm{Cl}(1)$ & $60(1)$ & $45(1)$ & $73(2)$ & $2(1)$ & $-16(1)$ & $4(1)$ \\
\hline $\mathrm{O}(1)$ & $56(4)$ & $56(4)$ & $71(5)$ & $7(4)$ & $-16(4)$ & $-2(3)$ \\
\hline $\mathrm{O}(2)$ & $64(4)$ & $46(4)$ & $62(5)$ & $-9(3)$ & $-27(4)$ & $4(3)$ \\
\hline $\mathrm{N}(1)$ & $56(5)$ & $41(4)$ & $55(6)$ & $3(4)$ & $-15(5)$ & $14(4)$ \\
\hline $\mathrm{N}(2)$ & $60(5)$ & $38(4)$ & $60(6)$ & $0(4)$ & $-19(4)$ & $6(4)$ \\
\hline $\mathrm{N}(3)$ & $69(6)$ & $41(5)$ & $51(6)$ & $-4(4)$ & $-29(5)$ & $8(4)$ \\
\hline $\mathrm{N}(4)$ & $90(8)$ & $64(6)$ & $154(12)$ & $47(7)$ & $-72(8)$ & $-25(6)$ \\
\hline$C(1)$ & $51(6)$ & $44(5)$ & $54(7)$ & $-5(4)$ & $-20(5)$ & $10(4)$ \\
\hline$C(2)$ & $39(5)$ & $39(5)$ & $70(7)$ & $2(5)$ & $-16(5)$ & $1(4)$ \\
\hline$C(3)$ & $46(6)$ & $53(6)$ & $59(7)$ & $5(5)$ & $-22(5)$ & $2(5)$ \\
\hline$C(4)$ & $43(5)$ & $47(5)$ & $55(6)$ & $-1(4)$ & $-16(5)$ & $-1(4)$ \\
\hline$C(5)$ & $45(5)$ & $53(6)$ & $60(7)$ & $3(5)$ & $-11(5)$ & $1(5)$ \\
\hline$C(6)$ & $49(5)$ & $47(5)$ & $53(6)$ & $-4(5)$ & $-10(5)$ & $-2(4)$ \\
\hline$C(7)$ & $56(6)$ & $45(5)$ & $49(6)$ & $13(4)$ & $-17(5)$ & $-7(4)$ \\
\hline$C(8)$ & $42(5)$ & $49(6)$ & $49(6)$ & $10(5)$ & $-14(5)$ & $1(4)$ \\
\hline$C(9)$ & $53(6)$ & $47(5)$ & $57(7)$ & $4(5)$ & $-19(5)$ & $-7(4)$ \\
\hline$C(10)$ & $43(5)$ & $56(5)$ & $46(6)$ & $3(4)$ & $-12(4)$ & $7(4)$ \\
\hline$C(11)$ & $44(5)$ & $51(5)$ & $48(6)$ & $1(5)$ & $-18(5)$ & $12(4)$ \\
\hline$C(12)$ & $49(6)$ & $53(6)$ & $63(8)$ & $2(5)$ & $-11(5)$ & $5(5)$ \\
\hline$C(13)$ & $65(7)$ & $48(5)$ & $51(7)$ & $-12(5)$ & $-21(6)$ & $-1(5)$ \\
\hline$C(14)$ & $56(6)$ & $48(6)$ & $59(8)$ & $-5(5)$ & $-23(6)$ & $11(5)$ \\
\hline$C(15)$ & $47(5)$ & $36(5)$ & $73(8)$ & $-2(5)$ & $-15(5)$ & $3(4)$ \\
\hline$C(16)$ & $40(5)$ & $36(5)$ & $75(7)$ & $-1(5)$ & $-19(5)$ & $2(4)$ \\
\hline$C(17)$ & $52(6)$ & $47(5)$ & $62(7)$ & $-2(5)$ & $-16(5)$ & $2(5)$ \\
\hline$C(18)$ & $51(6)$ & $48(5)$ & $52(6)$ & $-3(5)$ & $-15(5)$ & $5(4)$ \\
\hline$C(19)$ & $50(6)$ & $43(5)$ & $64(7)$ & $-9(5)$ & $-16(5)$ & $7(4)$ \\
\hline$C(20)$ & $60(6)$ & $39(5)$ & $63(7)$ & $2(5)$ & $-17(6)$ & $9(5)$ \\
\hline$C(21)$ & $60(6)$ & $36(5)$ & $83(9)$ & $10(5)$ & $-29(6)$ & $-9(5)$ \\
\hline$C(22)$ & 110(9) & $47(6)$ & $36(6)$ & $7(5)$ & $-22(6)$ & $-2(6)$ \\
\hline$C(23)$ & $121(11)$ & $51(7)$ & $84(9)$ & $12(7)$ & $-46(9)$ & $-19(8)$ \\
\hline$C(24)$ & $84(8)$ & $52(7)$ & $93(11)$ & $25(7)$ & $-41(8)$ & $-24(7)$ \\
\hline
\end{tabular}




\begin{tabular}{lcccccc}
$\mathrm{C}(25)$ & $61(7)$ & $55(6)$ & $81(9)$ & $12(6)$ & $-20(6)$ & $3(5)$ \\
$\mathrm{C}(26)$ & $77(7)$ & $53(6)$ & $63(8)$ & $11(5)$ & $-20(6)$ & $4(6)$ \\
$\mathrm{C}(27)$ & $100(10)$ & $57(7)$ & $100(11)$ & $24(7)$ & $-53(9)$ & $-25(7)$ \\
\hline
\end{tabular}

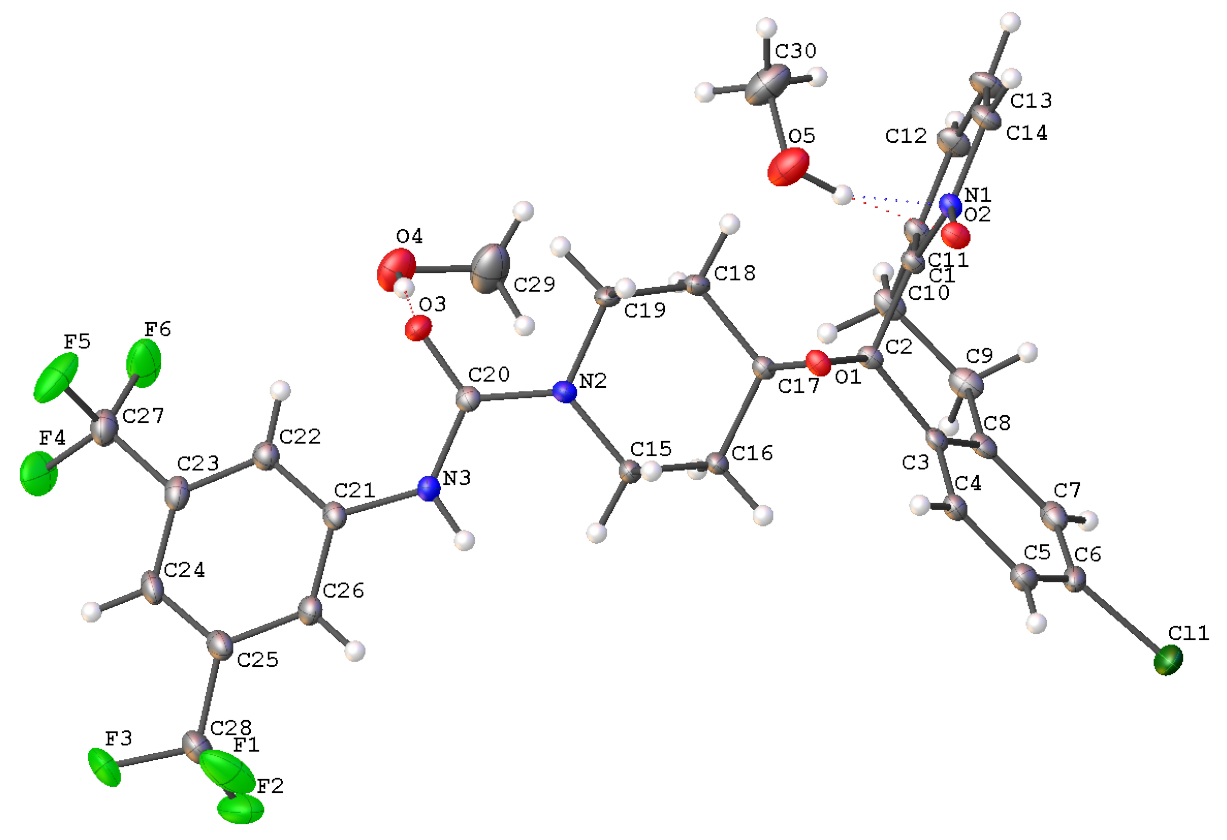

Figure S8. The complete numbering scheme of $\mathbf{8 h}$ with $50 \%$ thermal ellipsoid probability levels. The hydrogen atoms are shown as circles for clarity.

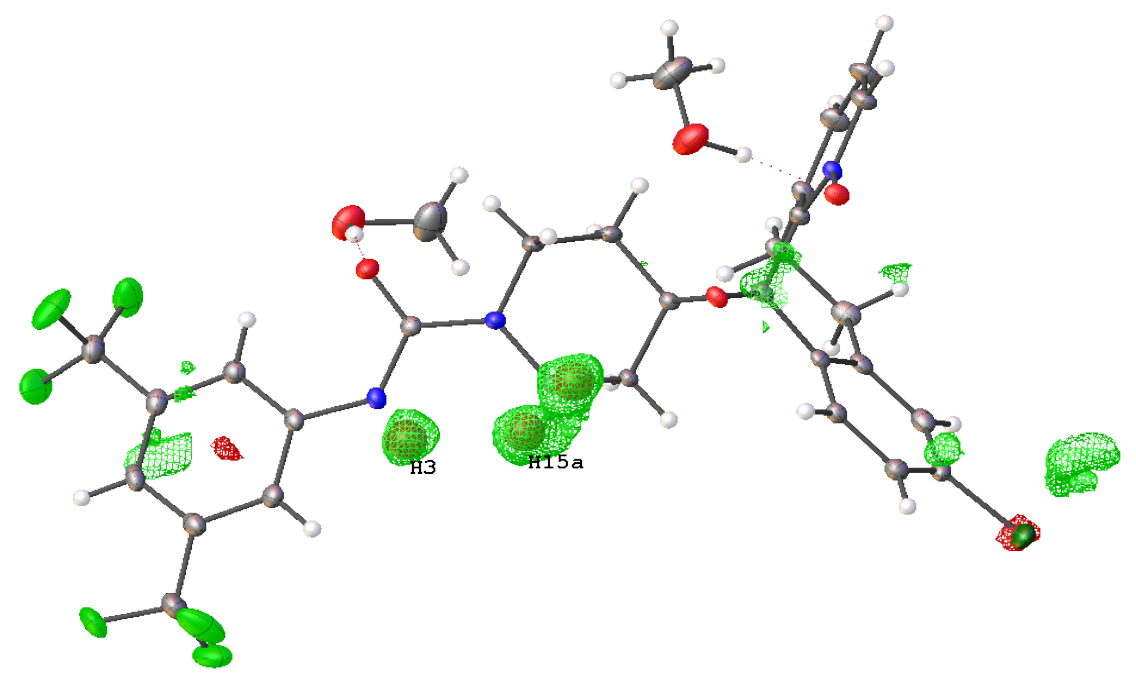

Figure S9. The Fourier difference map of total electron density in $\mathbf{8 h}\left(0.128 \mathrm{e} / \AA^{3}\right.$ surface $)$ excluding contributions from H3, H15a, and H15b. The close contact between H3 and H15a (1.909 $\AA$ ) in $\mathbf{8 h}$ is suggested by the difference map. 
Table S17. Crystal data and structure refinement for $\mathbf{8 h}$.

Identification code

Empirical formula

Formula weight

Temperature

Wavelength

Crystal system

Space group

Unit cell dimensions

Volume

Z

Density (calculated)

Absorption coefficient

$\mathrm{F}(000)$

Crystal size

Crystal color and habit

Diffractometer

Theta range for data collection

Index ranges

Reflections collected

Independent reflections

Observed reflections $(\mathrm{I}>2 \operatorname{sigma}(\mathrm{I}))$

Completeness to theta $=25.242^{\circ}$

Absorption correction

Max. and min. transmission

Solution method

Refinement method

Data / restraints / parameters

Goodness-of-fit on $\mathrm{F}^{2}$

Final R indices $[\mathrm{I}>2 \operatorname{sigma}(\mathrm{I})]$

$\mathrm{R}$ indices (all data)

Absolute structure parameter

Largest diff. peak and hole
1983156

$\mathrm{C}_{30} \mathrm{H}_{30} \mathrm{ClF}_{6} \mathrm{~N}_{3} \mathrm{O}_{5}$

662.02

93(2) K

$0.71073 \AA$

Orthorhombic

$\mathrm{P} 22_{1} 2_{1}$

$\mathrm{a}=8.3709(2) \AA \quad \alpha=90^{\circ}$

$\mathrm{b}=17.3851(4) \AA \quad \beta=90^{\circ}$

$c=20.8015(6) \AA \quad \gamma=90^{\circ}$

$3027.22(13) \AA^{3}$

4

$1.453 \mathrm{Mg} / \mathrm{m}^{3}$

$0.208 \mathrm{~mm}^{-1}$

1368

$0.200 \times 0.200 \times 0.200 \mathrm{~mm}^{3}$

colorless block

Dectris Pilatus 3R

2.873 to $27.479^{\circ}$

$-10<=\mathrm{h}<=9,-21<=\mathrm{k}<=22,-27<=\mathrm{l}<=26$

25701

$6891[\mathrm{R}($ int $)=0.0260]$

6536

$99.8 \%$

Semi-empirical from equivalents

1.00000 and 0.81649

SHELXT-2014/5 (Sheldrick, 2014)

SHELXL-2014/7 (Sheldrick, 2014)

$6891 / 0$ / 420

1.025

$\mathrm{R} 1=0.0294, \mathrm{wR} 2=0.0703$

$\mathrm{R} 1=0.0318, \mathrm{wR} 2=0.0714$

$0.017(18)$

0.230 and -0.225 e. $\AA^{-3}$ 
Table S18. Atomic coordinates $\left(\times 10^{4}\right)$ and equivalent isotropic displacement parameters $\left(\AA^{2} \times 10^{3}\right)$ for $8 \mathrm{~h}$. $\mathrm{U}(\mathrm{eq})$ is defined as one third of the trace of the orthogonalized $\mathrm{Uij}^{\mathrm{ij}}$ tensor.

\begin{tabular}{|c|c|c|c|c|}
\hline & $\mathrm{x}$ & $\mathrm{y}$ & $\mathrm{z}$ & $\mathrm{U}(\mathrm{eq})$ \\
\hline $\mathrm{Cl}(1)$ & $1133(1)$ & $-1163(1)$ & $5711(1)$ & $27(1)$ \\
\hline $\mathrm{F}(1)$ & $829(2)$ & $6457(1)$ & $6926(1)$ & $52(1)$ \\
\hline $\mathrm{F}(2)$ & $-1596(2)$ & $6187(1)$ & $6707(1)$ & $46(1)$ \\
\hline $\mathrm{F}(3)$ & $-784(2)$ & $7348(1)$ & $6624(1)$ & $46(1)$ \\
\hline $\mathrm{F}(4)$ & $-1395(2)$ & $7842(1)$ & $4327(1)$ & $42(1)$ \\
\hline $\mathrm{F}(5)$ & $1082(2)$ & $7864(1)$ & $4102(1)$ & $46(1)$ \\
\hline $\mathrm{F}(6)$ & $-454(2)$ & $7050(1)$ & $3638(1)$ & $46(1)$ \\
\hline $\mathrm{O}(1)$ & $4769(2)$ & $1924(1)$ & $4383(1)$ & $14(1)$ \\
\hline $\mathrm{O}(2)$ & $6751(2)$ & $941(1)$ & $3765(1)$ & $15(1)$ \\
\hline $\mathrm{O}(3)$ & $2830(2)$ & $5041(1)$ & $3914(1)$ & $19(1)$ \\
\hline $\mathrm{N}(1)$ & $5627(2)$ & $906(1)$ & $3317(1)$ & $13(1)$ \\
\hline $\mathrm{N}(2)$ & $3149(2)$ & $3874(1)$ & $4389(1)$ & $16(1)$ \\
\hline $\mathrm{N}(3)$ & $2098(2)$ & $4908(1)$ & $4966(1)$ & $15(1)$ \\
\hline $\mathrm{C}(1)$ & $4089(2)$ & $1108(1)$ & $3442(1)$ & $13(1)$ \\
\hline$C(2)$ & $3629(2)$ & $1424(1)$ & $4087(1)$ & $12(1)$ \\
\hline$C(3)$ & $2827(2)$ & $834(1)$ & $4509(1)$ & $13(1)$ \\
\hline$C(4)$ & $3427(2)$ & $723(1)$ & $5130(1)$ & $15(1)$ \\
\hline$C(5)$ & $2888(2)$ & $126(1)$ & $5516(1)$ & $19(1)$ \\
\hline$C(6)$ & $1730(2)$ & $-361(1)$ & $5268(1)$ & $19(1)$ \\
\hline$C(7)$ & $1061(2)$ & $-238(1)$ & $4671(1)$ & $19(1)$ \\
\hline$C(8)$ & $1582(2)$ & $367(1)$ & $4279(1)$ & $16(1)$ \\
\hline$C(9)$ & $703(2)$ & $451(1)$ & $3642(1)$ & $20(1)$ \\
\hline$C(10)$ & $1201(2)$ & $1104(1)$ & $3188(1)$ & $19(1)$ \\
\hline$C(11)$ & $2904(2)$ & $996(1)$ & 2979(1) & $16(1)$ \\
\hline$C(12)$ & $3324(2)$ & $728(1)$ & $2374(1)$ & $20(1)$ \\
\hline$C(13)$ & $4915(3)$ & $563(1)$ & $2246(1)$ & $22(1)$ \\
\hline$C(14)$ & $6041(2)$ & $643(1)$ & $2721(1)$ & $18(1)$ \\
\hline$C(15)$ & $2962(2)$ & $3330(1)$ & 4919(1) & $14(1)$ \\
\hline$C(16)$ & $2248(2)$ & $2575(1)$ & $4673(1)$ & $14(1)$ \\
\hline$C(17)$ & $3300(2)$ & $2266(1)$ & $4148(1)$ & $12(1)$ \\
\hline$C(18)$ & $3514(2)$ & $2824(1)$ & $3598(1)$ & $16(1)$ \\
\hline$C(19)$ & $4146(2)$ & $3591(1)$ & $3858(1)$ & $18(1)$ \\
\hline
\end{tabular}




$\begin{array}{lrlll}\mathrm{C}(20) & 2703(2) & 4629(1) & 4397(1) & 14(1) \\ \mathrm{C}(21) & 1356(2) & 5624(1) & 5062(1) & 15(1) \\ \mathrm{C}(22) & 1013(2) & 6156(1) & 4577(1) & 18(1) \\ \mathrm{C}(23) & 179(2) & 6824(1) & 4729(1) & 20(1) \\ \mathrm{C}(24) & -300(2) & 6991(1) & 5352(1) & 21(1) \\ \mathrm{C}(25) & 94(2) & 6469(1) & 5833(1) & 20(1) \\ \mathrm{C}(26) & 909(2) & 5792(1) & 5694(1) & 18(1) \\ \mathrm{C}(27) & -156(3) & 7390(1) & 4202(1) & 25(1) \\ \mathrm{C}(28) & -365(3) & 6620(1) & 6517(1) & 29(1) \\ \mathrm{O}(4) & 455(2) & 5060(1) & 3006(1) & 33(1) \\ \mathrm{C}(29) & 127(4) & 4279(2) & 2885(1) & 44(1) \\ \mathrm{O}(5) & 8289(2) & 2402(1) & 3639(1) & 36(1) \\ \mathrm{C}(30) & 8023(3) & 2552(2) & 2977(1) & 40(1)\end{array}$

Table S19. Bond lengths $[\AA]$ and angles $\left[^{\circ}\right]$ for $8 \mathbf{h}$.

$\begin{array}{ll}\mathrm{Cl}(1)-\mathrm{C}(6) & 1.7440(19) \\ \mathrm{F}(1)-\mathrm{C}(28) & 1.342(3) \\ \mathrm{F}(2)-\mathrm{C}(28) & 1.336(3) \\ \mathrm{F}(3)-\mathrm{C}(28) & 1.331(2) \\ \mathrm{F}(4)-\mathrm{C}(27) & 1.327(2) \\ \mathrm{F}(5)-\mathrm{C}(27) & 1.340(3) \\ \mathrm{F}(6)-\mathrm{C}(27) & 1.337(3) \\ \mathrm{O}(1)-\mathrm{C}(2) & 1.430(2) \\ \mathrm{O}(1)-\mathrm{C}(17) & 1.451(2) \\ \mathrm{O}(2)-\mathrm{N}(1) & 1.3256(19) \\ \mathrm{O}(3)-\mathrm{C}(20) & 1.239(2) \\ \mathrm{N}(1)-\mathrm{C}(1) & 1.361(2) \\ \mathrm{N}(1)-\mathrm{C}(14) & 1.365(2) \\ \mathrm{N}(2)-\mathrm{C}(20) & 1.364(2) \\ \mathrm{N}(2)-\mathrm{C}(15) & 1.462(2) \\ \mathrm{N}(2)-\mathrm{C}(19) & 1.470(2) \\ \mathrm{N}(3)-\mathrm{C}(20) & 1.376(2) \\ \mathrm{N}(3)-\mathrm{C}(21) & 1.406(2) \\ \mathrm{N}(3)-\mathrm{H}(3) & 0.92(2) \\ \mathrm{C}(1)-\mathrm{C}(11) & 1.397(3) \\ & \end{array}$




\begin{tabular}{|c|c|}
\hline$C(1)-C(2)$ & $1.499(2)$ \\
\hline$C(2)-C(17)$ & $1.495(2)$ \\
\hline$C(2)-C(3)$ & $1.508(3)$ \\
\hline$C(3)-C(4)$ & $1.400(3)$ \\
\hline$C(3)-C(8)$ & $1.405(3)$ \\
\hline$C(4)-C(5)$ & $1.386(3)$ \\
\hline $\mathrm{C}(4)-\mathrm{H}(4)$ & 0.9500 \\
\hline$C(5)-C(6)$ & $1.387(3)$ \\
\hline $\mathrm{C}(5)-\mathrm{H}(5)$ & 0.9500 \\
\hline$C(6)-C(7)$ & $1.379(3)$ \\
\hline$C(7)-C(8)$ & $1.401(3)$ \\
\hline $\mathrm{C}(7)-\mathrm{H}(7)$ & 0.9500 \\
\hline$C(8)-C(9)$ & $1.523(3)$ \\
\hline$C(9)-C(10)$ & $1.536(3)$ \\
\hline $\mathrm{C}(9)-\mathrm{H}(9 \mathrm{~A})$ & 0.9900 \\
\hline $\mathrm{C}(9)-\mathrm{H}(9 \mathrm{~B})$ & 0.9900 \\
\hline$C(10)-C(11)$ & $1.502(3)$ \\
\hline $\mathrm{C}(10)-\mathrm{H}(10 \mathrm{~A})$ & 0.9900 \\
\hline $\mathrm{C}(10)-\mathrm{H}(10 \mathrm{~B})$ & 0.9900 \\
\hline$C(11)-C(12)$ & $1.386(3)$ \\
\hline$C(12)-C(13)$ & $1.388(3)$ \\
\hline $\mathrm{C}(12)-\mathrm{H}(12)$ & 0.9500 \\
\hline$C(13)-C(14)$ & $1.373(3)$ \\
\hline $\mathrm{C}(13)-\mathrm{H}(13)$ & 0.9500 \\
\hline $\mathrm{C}(14)-\mathrm{H}(14)$ & 0.9500 \\
\hline$C(15)-C(16)$ & $1.531(2)$ \\
\hline $\mathrm{C}(15)-\mathrm{H}(15 \mathrm{~A})$ & 0.9900 \\
\hline $\mathrm{C}(15)-\mathrm{H}(15 \mathrm{~B})$ & 0.9900 \\
\hline$C(16)-C(17)$ & $1.502(2)$ \\
\hline$C(16)-H(16 A)$ & 0.9900 \\
\hline $\mathrm{C}(16)-\mathrm{H}(16 \mathrm{~B})$ & 0.9900 \\
\hline$C(17)-C(18)$ & $1.511(2)$ \\
\hline$C(18)-C(19)$ & $1.533(3)$ \\
\hline $\mathrm{C}(18)-\mathrm{H}(18 \mathrm{~A})$ & 0.9900 \\
\hline $\mathrm{C}(18)-\mathrm{H}(18 \mathrm{~B})$ & 0.9900 \\
\hline $\mathrm{C}(19)-\mathrm{H}(19 \mathrm{~A})$ & 0.9900 \\
\hline
\end{tabular}




\begin{tabular}{|c|c|}
\hline $\mathrm{C}(19)-\mathrm{H}(19 \mathrm{~B})$ & 0.9900 \\
\hline$C(21)-C(22)$ & $1.398(3)$ \\
\hline$C(21)-C(26)$ & $1.399(3)$ \\
\hline$C(22)-C(23)$ & $1.390(3)$ \\
\hline $\mathrm{C}(22)-\mathrm{H}(22)$ & 0.9500 \\
\hline$C(23)-C(24)$ & $1.386(3)$ \\
\hline$C(23)-C(27)$ & $1.501(3)$ \\
\hline$C(24)-C(25)$ & $1.391(3)$ \\
\hline $\mathrm{C}(24)-\mathrm{H}(24)$ & 0.9500 \\
\hline$C(25)-C(26)$ & $1.390(3)$ \\
\hline$C(25)-C(28)$ & $1.498(3)$ \\
\hline $\mathrm{C}(26)-\mathrm{H}(26)$ & 0.9500 \\
\hline $\mathrm{O}(4)-\mathrm{C}(29)$ & $1.408(3)$ \\
\hline $\mathrm{O}(4)-\mathrm{H}(4 \mathrm{~A})$ & $0.84(3)$ \\
\hline $\mathrm{C}(29)-\mathrm{H}(29 \mathrm{~A})$ & 0.9800 \\
\hline C(29)-H(29B) & 0.9800 \\
\hline $\mathrm{C}(29)-\mathrm{H}(29 \mathrm{C})$ & 0.9800 \\
\hline $\mathrm{O}(5)-\mathrm{C}(30)$ & $1.419(3)$ \\
\hline $\mathrm{O}(5)-\mathrm{H}(5 \mathrm{~A})$ & $0.96(4)$ \\
\hline $\mathrm{C}(30)-\mathrm{H}(30 \mathrm{~A})$ & 0.9800 \\
\hline $\mathrm{C}(30)-\mathrm{H}(30 \mathrm{~B})$ & 0.9800 \\
\hline $\mathrm{C}(30)-\mathrm{H}(30 \mathrm{C})$ & 0.9800 \\
\hline $\mathrm{C}(2)-\mathrm{O}(1)-\mathrm{C}(17)$ & $62.50(11)$ \\
\hline $\mathrm{O}(2)-\mathrm{N}(1)-\mathrm{C}(1)$ & $121.64(14)$ \\
\hline $\mathrm{O}(2)-\mathrm{N}(1)-\mathrm{C}(14)$ & $118.27(15)$ \\
\hline $\mathrm{C}(1)-\mathrm{N}(1)-\mathrm{C}(14)$ & $120.08(16)$ \\
\hline $\mathrm{C}(20)-\mathrm{N}(2)-\mathrm{C}(15)$ & $125.70(15)$ \\
\hline $\mathrm{C}(20)-\mathrm{N}(2)-\mathrm{C}(19)$ & $119.14(15)$ \\
\hline $\mathrm{C}(15)-\mathrm{N}(2)-\mathrm{C}(19)$ & $114.32(14)$ \\
\hline $\mathrm{C}(20)-\mathrm{N}(3)-\mathrm{C}(21)$ & $126.66(16)$ \\
\hline $\mathrm{C}(20)-\mathrm{N}(3)-\mathrm{H}(3)$ & $122.2(14)$ \\
\hline $\mathrm{C}(21)-\mathrm{N}(3)-\mathrm{H}(3)$ & $110.9(14)$ \\
\hline $\mathrm{N}(1)-\mathrm{C}(1)-\mathrm{C}(11)$ & $120.21(16)$ \\
\hline$N(1)-C(1)-C(2)$ & $120.65(15)$ \\
\hline$C(11)-C(1)-C(2)$ & $119.09(16)$ \\
\hline
\end{tabular}




\begin{tabular}{|c|c|}
\hline $\mathrm{O}(1)-\mathrm{C}(2)-\mathrm{C}(17)$ & $59.42(11)$ \\
\hline $\mathrm{O}(1)-\mathrm{C}(2)-\mathrm{C}(1)$ & $115.92(15)$ \\
\hline$C(17)-C(2)-C(1)$ & $118.89(15)$ \\
\hline $\mathrm{O}(1)-\mathrm{C}(2)-\mathrm{C}(3)$ & $117.28(15)$ \\
\hline$C(17)-C(2)-C(3)$ & $122.27(15)$ \\
\hline$C(1)-C(2)-C(3)$ & $112.71(14)$ \\
\hline$C(4)-C(3)-C(8)$ & $119.99(17)$ \\
\hline$C(4)-C(3)-C(2)$ & $118.20(16)$ \\
\hline$C(8)-C(3)-C(2)$ & $121.68(16)$ \\
\hline$C(5)-C(4)-C(3)$ & $121.43(17)$ \\
\hline $\mathrm{C}(5)-\mathrm{C}(4)-\mathrm{H}(4)$ & 119.3 \\
\hline $\mathrm{C}(3)-\mathrm{C}(4)-\mathrm{H}(4)$ & 119.3 \\
\hline$C(4)-C(5)-C(6)$ & $118.00(18)$ \\
\hline $\mathrm{C}(4)-\mathrm{C}(5)-\mathrm{H}(5)$ & 121.0 \\
\hline $\mathrm{C}(6)-\mathrm{C}(5)-\mathrm{H}(5)$ & 121.0 \\
\hline$C(7)-C(6)-C(5)$ & $121.57(18)$ \\
\hline $\mathrm{C}(7)-\mathrm{C}(6)-\mathrm{Cl}(1)$ & $118.89(15)$ \\
\hline $\mathrm{C}(5)-\mathrm{C}(6)-\mathrm{Cl}(1)$ & $119.52(16)$ \\
\hline$C(6)-C(7)-C(8)$ & $120.95(18)$ \\
\hline $\mathrm{C}(6)-\mathrm{C}(7)-\mathrm{H}(7)$ & 119.5 \\
\hline $\mathrm{C}(8)-\mathrm{C}(7)-\mathrm{H}(7)$ & 119.5 \\
\hline$C(7)-C(8)-C(3)$ & $117.85(18)$ \\
\hline$C(7)-C(8)-C(9)$ & $115.32(17)$ \\
\hline$C(3)-C(8)-C(9)$ & $126.82(17)$ \\
\hline$C(8)-C(9)-C(10)$ & $118.35(16)$ \\
\hline $\mathrm{C}(8)-\mathrm{C}(9)-\mathrm{H}(9 \mathrm{~A})$ & 107.7 \\
\hline $\mathrm{C}(10)-\mathrm{C}(9)-\mathrm{H}(9 \mathrm{~A})$ & 107.7 \\
\hline $\mathrm{C}(8)-\mathrm{C}(9)-\mathrm{H}(9 \mathrm{~B})$ & 107.7 \\
\hline $\mathrm{C}(10)-\mathrm{C}(9)-\mathrm{H}(9 \mathrm{~B})$ & 107.7 \\
\hline $\mathrm{H}(9 \mathrm{~A})-\mathrm{C}(9)-\mathrm{H}(9 \mathrm{~B})$ & 107.1 \\
\hline$C(11)-C(10)-C(9)$ & $110.03(16)$ \\
\hline $\mathrm{C}(11)-\mathrm{C}(10)-\mathrm{H}(10 \mathrm{~A})$ & 109.7 \\
\hline $\mathrm{C}(9)-\mathrm{C}(10)-\mathrm{H}(10 \mathrm{~A})$ & 109.7 \\
\hline $\mathrm{C}(11)-\mathrm{C}(10)-\mathrm{H}(10 \mathrm{~B})$ & 109.7 \\
\hline $\mathrm{C}(9)-\mathrm{C}(10)-\mathrm{H}(10 \mathrm{~B})$ & 109.7 \\
\hline $\mathrm{H}(10 \mathrm{~A})-\mathrm{C}(10)-\mathrm{H}(10 \mathrm{~B})$ & 108.2 \\
\hline
\end{tabular}




\begin{tabular}{|c|c|}
\hline$C(12)-C(11)-C(1)$ & $119.56(18)$ \\
\hline$C(12)-C(11)-C(10)$ & $123.05(17)$ \\
\hline$C(1)-C(11)-C(10)$ & $117.14(16)$ \\
\hline$C(11)-C(12)-C(13)$ & $119.11(18)$ \\
\hline $\mathrm{C}(11)-\mathrm{C}(12)-\mathrm{H}(12)$ & 120.4 \\
\hline $\mathrm{C}(13)-\mathrm{C}(12)-\mathrm{H}(12)$ & 120.4 \\
\hline$C(14)-C(13)-C(12)$ & $120.01(18)$ \\
\hline $\mathrm{C}(14)-\mathrm{C}(13)-\mathrm{H}(13)$ & 120.0 \\
\hline $\mathrm{C}(12)-\mathrm{C}(13)-\mathrm{H}(13)$ & 120.0 \\
\hline $\mathrm{N}(1)-\mathrm{C}(14)-\mathrm{C}(13)$ & $120.83(19)$ \\
\hline $\mathrm{N}(1)-\mathrm{C}(14)-\mathrm{H}(14)$ & 119.6 \\
\hline $\mathrm{C}(13)-\mathrm{C}(14)-\mathrm{H}(14)$ & 119.6 \\
\hline $\mathrm{N}(2)-\mathrm{C}(15)-\mathrm{C}(16)$ & $110.17(15)$ \\
\hline $\mathrm{N}(2)-\mathrm{C}(15)-\mathrm{H}(15 \mathrm{~A})$ & 109.6 \\
\hline $\mathrm{C}(16)-\mathrm{C}(15)-\mathrm{H}(15 \mathrm{~A})$ & 109.6 \\
\hline $\mathrm{N}(2)-\mathrm{C}(15)-\mathrm{H}(15 \mathrm{~B})$ & 109.6 \\
\hline $\mathrm{C}(16)-\mathrm{C}(15)-\mathrm{H}(15 \mathrm{~B})$ & 109.6 \\
\hline $\mathrm{H}(15 \mathrm{~A})-\mathrm{C}(15)-\mathrm{H}(15 \mathrm{~B})$ & 108.1 \\
\hline$C(17)-C(16)-C(15)$ & $108.70(15)$ \\
\hline$C(17)-C(16)-H(16 A)$ & 110.0 \\
\hline$C(15)-C(16)-H(16 A)$ & 110.0 \\
\hline $\mathrm{C}(17)-\mathrm{C}(16)-\mathrm{H}(16 \mathrm{~B})$ & 110.0 \\
\hline$C(15)-C(16)-H(16 B)$ & 110.0 \\
\hline $\mathrm{H}(16 \mathrm{~A})-\mathrm{C}(16)-\mathrm{H}(16 \mathrm{~B})$ & 108.3 \\
\hline $\mathrm{O}(1)-\mathrm{C}(17)-\mathrm{C}(2)$ & $58.08(10)$ \\
\hline $\mathrm{O}(1)-\mathrm{C}(17)-\mathrm{C}(16)$ & $113.49(14)$ \\
\hline$C(2)-C(17)-C(16)$ & $121.35(15)$ \\
\hline $\mathrm{O}(1)-\mathrm{C}(17)-\mathrm{C}(18)$ & $114.72(14)$ \\
\hline$C(2)-C(17)-C(18)$ & $122.78(15)$ \\
\hline$C(16)-C(17)-C(18)$ & $113.02(15)$ \\
\hline$C(17)-C(18)-C(19)$ & $109.41(14)$ \\
\hline $\mathrm{C}(17)-\mathrm{C}(18)-\mathrm{H}(18 \mathrm{~A})$ & 109.8 \\
\hline $\mathrm{C}(19)-\mathrm{C}(18)-\mathrm{H}(18 \mathrm{~A})$ & 109.8 \\
\hline $\mathrm{C}(17)-\mathrm{C}(18)-\mathrm{H}(18 \mathrm{~B})$ & 109.8 \\
\hline $\mathrm{C}(19)-\mathrm{C}(18)-\mathrm{H}(18 \mathrm{~B})$ & 109.8 \\
\hline $\mathrm{H}(18 \mathrm{~A})-\mathrm{C}(18)-\mathrm{H}(18 \mathrm{~B})$ & 108.2 \\
\hline
\end{tabular}




$\begin{array}{ll}\mathrm{N}(2)-\mathrm{C}(19)-\mathrm{C}(18) & 111.13(15) \\ \mathrm{N}(2)-\mathrm{C}(19)-\mathrm{H}(19 \mathrm{~A}) & 109.4 \\ \mathrm{C}(18)-\mathrm{C}(19)-\mathrm{H}(19 \mathrm{~A}) & 109.4 \\ \mathrm{~N}(2)-\mathrm{C}(19)-\mathrm{H}(19 \mathrm{~B}) & 109.4 \\ \mathrm{C}(18)-\mathrm{C}(19)-\mathrm{H}(19 \mathrm{~B}) & 109.4 \\ \mathrm{H}(19 \mathrm{~A})-\mathrm{C}(19)-\mathrm{H}(19 \mathrm{~B}) & 108.0 \\ \mathrm{O}(3)-\mathrm{C}(20)-\mathrm{N}(2) & 121.56(16) \\ \mathrm{O}(3)-\mathrm{C}(20)-\mathrm{N}(3) & 121.68(16) \\ \mathrm{N}(2)-\mathrm{C}(20)-\mathrm{N}(3) & 116.77(16) \\ \mathrm{C}(22)-\mathrm{C}(21)-\mathrm{C}(26) & 119.00(17) \\ \mathrm{C}(22)-\mathrm{C}(21)-\mathrm{N}(3) & 125.09(17) \\ \mathrm{C}(26)-\mathrm{C}(21)-\mathrm{N}(3) & 115.90(17) \\ \mathrm{C}(23)-\mathrm{C}(22)-\mathrm{C}(21) & 119.47(18) \\ \mathrm{C}(23)-\mathrm{C}(22)-\mathrm{H}(22) & 120.3 \\ \mathrm{C}(21)-\mathrm{C}(22)-\mathrm{H}(22) & 120.3 \\ \mathrm{C}(24)-\mathrm{C}(23)-\mathrm{C}(22) & 122.17(18) \\ \mathrm{C}(24)-\mathrm{C}(23)-\mathrm{C}(27) & 119.40(17) \\ \mathrm{C}(22)-\mathrm{C}(23)-\mathrm{C}(27) & 118.40(18) \\ \mathrm{C}(23)-\mathrm{C}(24)-\mathrm{C}(25) & 117.81(18) \\ \mathrm{C}(23)-\mathrm{C}(24)-\mathrm{H}(24) & 121.1 \\ \mathrm{C}(25)-\mathrm{C}(24)-\mathrm{H}(24) & 121.1 \\ \mathrm{C}(26)-\mathrm{C}(25)-\mathrm{C}(24) & 121.33(18) \\ \mathrm{C}(26)-\mathrm{C}(25)-\mathrm{C}(28) & 118.10(18) \\ \mathrm{C}(24)-\mathrm{C}(25)-\mathrm{C}(28) & 120.57(18) \\ \mathrm{C}(25)-\mathrm{C}(26)-\mathrm{C}(21) & 120.17(18) \\ \mathrm{C}(25)-\mathrm{C}(26)-\mathrm{H}(26) & 119.9 \\ \mathrm{C}(21)-\mathrm{C}(26)-\mathrm{H}(26) & 119.9 \\ \mathrm{~F}(4)-\mathrm{C}(27)-\mathrm{F}(6) & 106.77(18) \\ \mathrm{F}(4)-\mathrm{C}(27)-\mathrm{F}(5) & 105.72(17) \\ \mathrm{F}(6)-\mathrm{C}(27)-\mathrm{F}(5) & 106.30(19) \\ \mathrm{F}(4)-\mathrm{C}(27)-\mathrm{C}(23) & 113.05(18) \\ \mathrm{F}(6)-\mathrm{C}(27)-\mathrm{C}(23) & 112.64(16) \\ \mathrm{F}(5)-\mathrm{C}(27)-\mathrm{C}(23) & 111.84(18) \\ & 106.47(19) \\ \mathrm{F}(28)-\mathrm{F}(1) & 106.9(2) \\ & 105.51(19)\end{array}$




$\begin{array}{ll}\mathrm{F}(3)-\mathrm{C}(28)-\mathrm{C}(25) & 113.10(19) \\ \mathrm{F}(2)-\mathrm{C}(28)-\mathrm{C}(25) & 112.34(19) \\ \mathrm{F}(1)-\mathrm{C}(28)-\mathrm{C}(25) & 111.97(19) \\ \mathrm{C}(29)-\mathrm{O}(4)-\mathrm{H}(4 \mathrm{~A}) & 104(2) \\ \mathrm{O}(4)-\mathrm{C}(29)-\mathrm{H}(29 \mathrm{~A}) & 109.5 \\ \mathrm{O}(4)-\mathrm{C}(29)-\mathrm{H}(29 \mathrm{~B}) & 109.5 \\ \mathrm{H}(29 \mathrm{~A})-\mathrm{C}(29)-\mathrm{H}(29 \mathrm{~B}) & 109.5 \\ \mathrm{O}(4)-\mathrm{C}(29)-\mathrm{H}(29 \mathrm{C}) & 109.5 \\ \mathrm{H}(29 \mathrm{~A})-\mathrm{C}(29)-\mathrm{H}(29 \mathrm{C}) & 109.5 \\ \mathrm{H}(29 \mathrm{~B})-\mathrm{C}(29)-\mathrm{H}(29 \mathrm{C}) & 109.5 \\ \mathrm{C}(30)-\mathrm{O}(5)-\mathrm{H}(5 \mathrm{~A}) & 103(3) \\ \mathrm{O}(5)-\mathrm{C}(30)-\mathrm{H}(30 \mathrm{~A}) & 109.5 \\ \mathrm{O}(5)-\mathrm{C}(30)-\mathrm{H}(30 \mathrm{~B}) & 109.5 \\ \mathrm{H}(30 \mathrm{~A})-\mathrm{C}(30)-\mathrm{H}(30 \mathrm{~B}) & 109.5 \\ \mathrm{O}(5)-\mathrm{C}(30)-\mathrm{H}(30 \mathrm{C}) & 109.5 \\ \mathrm{H}(30 \mathrm{~A})-\mathrm{C}(30)-\mathrm{H}(30 \mathrm{C}) & 109.5 \\ \mathrm{H}(30 \mathrm{~B})-\mathrm{C}(30)-\mathrm{H}(30 \mathrm{C}) & 109.5\end{array}$

Table S20. Anisotropic displacement parameters $\left(\AA^{2} \times 10^{3}\right)$ for $\mathbf{8 h}$. The anisotropic displacement factor exponent takes the form: $-2 \pi^{2}\left[h^{2} a^{* 2} U^{11}+\ldots+2 h k a^{*} b^{*} U^{12}\right]$.

\begin{tabular}{lcccccc}
\hline & $\mathrm{U}^{11}$ & $\mathrm{U}^{22}$ & $\mathrm{U}^{33}$ & $\mathrm{U}^{23}$ & $\mathrm{U}^{13}$ & $\mathrm{U}^{12}$ \\
\hline $\mathrm{Cl}(1)$ & $36(1)$ & $17(1)$ & $29(1)$ & $2(1)$ & $11(1)$ & $-4(1)$ \\
$\mathrm{F}(1)$ & $57(1)$ & $74(1)$ & $27(1)$ & $-20(1)$ & $-6(1)$ & $23(1)$ \\
$\mathrm{F}(2)$ & $59(1)$ & $43(1)$ & $36(1)$ & $-3(1)$ & $26(1)$ & $-9(1)$ \\
$\mathrm{F}(3)$ & $76(1)$ & $25(1)$ & $38(1)$ & $-11(1)$ & $19(1)$ & $12(1)$ \\
$\mathrm{F}(4)$ & $38(1)$ & $36(1)$ & $51(1)$ & $14(1)$ & $7(1)$ & $22(1)$ \\
$\mathrm{F}(5)$ & $34(1)$ & $41(1)$ & $63(1)$ & $31(1)$ & $-1(1)$ & $-7(1)$ \\
$\mathrm{F}(6)$ & $80(1)$ & $28(1)$ & $30(1)$ & $4(1)$ & $-14(1)$ & $12(1)$ \\
$\mathrm{O}(1)$ & $12(1)$ & $14(1)$ & $14(1)$ & $-4(1)$ & $-2(1)$ & $1(1)$ \\
$\mathrm{O}(2)$ & $13(1)$ & $19(1)$ & $14(1)$ & $-1(1)$ & $-2(1)$ & $3(1)$ \\
$\mathrm{O}(3)$ & $22(1)$ & $16(1)$ & $19(1)$ & $3(1)$ & $5(1)$ & $1(1)$ \\
$\mathrm{N}(1)$ & $14(1)$ & $12(1)$ & $13(1)$ & $-2(1)$ & $-1(1)$ & $2(1)$ \\
$\mathrm{N}(2)$ & $21(1)$ & $14(1)$ & $14(1)$ & $0(1)$ & $6(1)$ & $1(1)$ \\
$\mathrm{N}(3)$ & $18(1)$ & $12(1)$ & $16(1)$ & $1(1)$ & $2(1)$ & $2(1)$
\end{tabular}




\begin{tabular}{|c|c|c|c|c|c|c|}
\hline$C(1)$ & $15(1)$ & $13(1)$ & $11(1)$ & $-2(1)$ & $0(1)$ & $1(1)$ \\
\hline$C(2)$ & $10(1)$ & $15(1)$ & $12(1)$ & $-2(1)$ & $-1(1)$ & $1(1)$ \\
\hline$C(3)$ & $12(1)$ & $12(1)$ & $15(1)$ & $-2(1)$ & $3(1)$ & $4(1)$ \\
\hline$C(4)$ & $17(1)$ & $14(1)$ & $16(1)$ & $-2(1)$ & $1(1)$ & $2(1)$ \\
\hline$C(5)$ & $21(1)$ & $18(1)$ & $19(1)$ & $-1(1)$ & $2(1)$ & $3(1)$ \\
\hline$C(6)$ & $22(1)$ & $12(1)$ & $23(1)$ & $1(1)$ & $9(1)$ & $1(1)$ \\
\hline$C(7)$ & $15(1)$ & $17(1)$ & $25(1)$ & $-5(1)$ & $4(1)$ & $-1(1)$ \\
\hline$C(8)$ & $13(1)$ & $18(1)$ & $18(1)$ & $-4(1)$ & $3(1)$ & $3(1)$ \\
\hline$C(9)$ & $13(1)$ & $26(1)$ & $22(1)$ & $-6(1)$ & $-1(1)$ & $-2(1)$ \\
\hline$C(10)$ & $14(1)$ & $24(1)$ & $18(1)$ & $-5(1)$ & $-4(1)$ & $4(1)$ \\
\hline$C(11)$ & $17(1)$ & $17(1)$ & $15(1)$ & $-3(1)$ & $-2(1)$ & $3(1)$ \\
\hline$C(12)$ & $22(1)$ & $26(1)$ & $13(1)$ & $-6(1)$ & $-4(1)$ & $1(1)$ \\
\hline$C(13)$ & $26(1)$ & $28(1)$ & $12(1)$ & $-7(1)$ & $1(1)$ & $3(1)$ \\
\hline$C(14)$ & $18(1)$ & $21(1)$ & $16(1)$ & $-5(1)$ & $4(1)$ & $3(1)$ \\
\hline$C(15)$ & $18(1)$ & $12(1)$ & $14(1)$ & $0(1)$ & $3(1)$ & $2(1)$ \\
\hline$C(16)$ & $17(1)$ & $13(1)$ & $14(1)$ & $-1(1)$ & $4(1)$ & $2(1)$ \\
\hline$C(17)$ & $12(1)$ & $13(1)$ & $12(1)$ & $-1(1)$ & $0(1)$ & $1(1)$ \\
\hline$C(18)$ & $19(1)$ & $17(1)$ & $12(1)$ & $1(1)$ & $4(1)$ & $3(1)$ \\
\hline$C(19)$ & $22(1)$ & $16(1)$ & $15(1)$ & $2(1)$ & $9(1)$ & $2(1)$ \\
\hline$C(20)$ & $11(1)$ & $14(1)$ & $16(1)$ & $-1(1)$ & $0(1)$ & $-2(1)$ \\
\hline$C(21)$ & $13(1)$ & $12(1)$ & $20(1)$ & $-2(1)$ & $1(1)$ & $-2(1)$ \\
\hline$C(22)$ & $18(1)$ & $15(1)$ & $20(1)$ & $-1(1)$ & $1(1)$ & $-1(1)$ \\
\hline$C(23)$ & $16(1)$ & $14(1)$ & $30(1)$ & $1(1)$ & $0(1)$ & $0(1)$ \\
\hline$C(24)$ & 19(1) & $12(1)$ & $31(1)$ & $-4(1)$ & $5(1)$ & $1(1)$ \\
\hline$C(25)$ & $21(1)$ & $15(1)$ & $24(1)$ & $-4(1)$ & $4(1)$ & $-2(1)$ \\
\hline$C(26)$ & $20(1)$ & $13(1)$ & $20(1)$ & $-1(1)$ & $1(1)$ & $-2(1)$ \\
\hline$C(27)$ & $24(1)$ & $17(1)$ & $33(1)$ & $1(1)$ & $1(1)$ & $3(1)$ \\
\hline$C(28)$ & $36(1)$ & $22(1)$ & $28(1)$ & $-5(1)$ & $7(1)$ & $6(1)$ \\
\hline $\mathrm{O}(4)$ & $35(1)$ & $32(1)$ & $33(1)$ & $9(1)$ & $-10(1)$ & $1(1)$ \\
\hline$C(29)$ & $59(2)$ & $34(1)$ & $38(1)$ & $6(1)$ & $-16(1)$ & $-1(1)$ \\
\hline $\mathrm{O}(5)$ & $43(1)$ & $34(1)$ & $29(1)$ & $6(1)$ & $-2(1)$ & $-12(1)$ \\
\hline$C(30)$ & $44(1)$ & $42(1)$ & $36(1)$ & $16(1)$ & $-3(1)$ & $-10(1)$ \\
\hline
\end{tabular}


Table S21. Hydrogen coordinates $\left(\mathrm{x} 10^{4}\right)$ and isotropic displacement parameters $\left(\AA^{2} \times 10^{3}\right)$ for $\mathbf{8 h}$.

\begin{tabular}{|c|c|c|c|c|}
\hline & $\mathrm{x}$ & $\mathrm{y}$ & $\mathrm{z}$ & $\mathrm{U}(\mathrm{eq})$ \\
\hline $\mathrm{H}(4)$ & 4220 & 1063 & 5291 & 19 \\
\hline $\mathrm{H}(5)$ & 3300 & 54 & 5937 & 23 \\
\hline $\mathrm{H}(7)$ & 236 & -569 & 4524 & 23 \\
\hline $\mathrm{H}(9 \mathrm{~A})$ & -447 & 516 & 3739 & 25 \\
\hline $\mathrm{H}(9 \mathrm{~B})$ & 818 & -40 & 3405 & 25 \\
\hline $\mathrm{H}(10 \mathrm{~A})$ & 494 & 1109 & 2806 & 22 \\
\hline $\mathrm{H}(10 \mathrm{~B})$ & 1089 & 1605 & 3410 & 22 \\
\hline $\mathrm{H}(12)$ & 2534 & 660 & 2051 & 24 \\
\hline $\mathrm{H}(13)$ & 5224 & 395 & 1830 & 26 \\
\hline $\mathrm{H}(14)$ & 7123 & 513 & 2634 & 22 \\
\hline $\mathrm{H}(15 \mathrm{~A})$ & 2252 & 3554 & 5251 & 17 \\
\hline $\mathrm{H}(15 \mathrm{~B})$ & 4015 & 3230 & 5119 & 17 \\
\hline $\mathrm{H}(16 \mathrm{~A})$ & 2178 & 2198 & 5029 & 17 \\
\hline $\mathrm{H}(16 \mathrm{~B})$ & 1157 & 2665 & 4505 & 17 \\
\hline $\mathrm{H}(18 \mathrm{~A})$ & 2479 & 2906 & 3378 & 19 \\
\hline $\mathrm{H}(18 \mathrm{~B})$ & 4278 & 2609 & 3282 & 19 \\
\hline $\mathrm{H}(19 \mathrm{~A})$ & 5257 & 3522 & 4010 & 21 \\
\hline $\mathrm{H}(19 \mathrm{~B})$ & 4157 & 3976 & 3507 & 21 \\
\hline $\mathrm{H}(22)$ & 1346 & 6063 & 4148 & 21 \\
\hline $\mathrm{H}(24)$ & -878 & 7447 & 5446 & 25 \\
\hline $\mathrm{H}(26)$ & 1164 & 5443 & 6030 & 21 \\
\hline $\mathrm{H}(29 \mathrm{~A})$ & 965 & 4064 & 2610 & 65 \\
\hline $\mathrm{H}(29 \mathrm{~B})$ & -908 & 4233 & 2668 & 65 \\
\hline $\mathrm{H}(29 \mathrm{C})$ & 94 & 3997 & 3292 & 65 \\
\hline $\mathrm{H}(30 \mathrm{~A})$ & 9050 & 2630 & 2761 & 61 \\
\hline $\mathrm{H}(30 \mathrm{~B})$ & 7368 & 3017 & 2932 & 61 \\
\hline $\mathrm{H}(30 \mathrm{C})$ & 7466 & 2115 & 2782 & 61 \\
\hline $\mathrm{H}(3)$ & $2050(30)$ & $4615(13)$ & $5331(11)$ & $15(5)$ \\
\hline $\mathrm{H}(4 \mathrm{~A})$ & $1180(40)$ & $5046(17)$ & $3286(15)$ & $36(7)$ \\
\hline $\mathrm{H}(5 \mathrm{~A})$ & $7730(50)$ & $1930(20)$ & $3710(20)$ & $82(13)$ \\
\hline
\end{tabular}


Table S22. Torsion angles $\left[^{\circ}\right]$ for $\mathbf{8 h}$.

\begin{tabular}{|c|c|}
\hline $\mathrm{O}(2)-\mathrm{N}(1)-\mathrm{C}(1)-\mathrm{C}(11)$ & $-173.89(16)$ \\
\hline$C(14)-N(1)-C(1)-C(11)$ & $4.7(3)$ \\
\hline $\mathrm{O}(2)-\mathrm{N}(1)-\mathrm{C}(1)-\mathrm{C}(2)$ & $3.7(3)$ \\
\hline $\mathrm{C}(14)-\mathrm{N}(1)-\mathrm{C}(1)-\mathrm{C}(2)$ & $-177.79(16)$ \\
\hline $\mathrm{C}(17)-\mathrm{O}(1)-\mathrm{C}(2)-\mathrm{C}(1)$ & $109.68(17)$ \\
\hline $\mathrm{C}(17)-\mathrm{O}(1)-\mathrm{C}(2)-\mathrm{C}(3)$ & $-113.14(17)$ \\
\hline $\mathrm{N}(1)-\mathrm{C}(1)-\mathrm{C}(2)-\mathrm{O}(1)$ & $37.4(2)$ \\
\hline $\mathrm{C}(11)-\mathrm{C}(1)-\mathrm{C}(2)-\mathrm{O}(1)$ & $-145.03(16)$ \\
\hline $\mathrm{N}(1)-\mathrm{C}(1)-\mathrm{C}(2)-\mathrm{C}(17)$ & $105.2(2)$ \\
\hline$C(11)-C(1)-C(2)-C(17)$ & $-77.2(2)$ \\
\hline $\mathrm{N}(1)-\mathrm{C}(1)-\mathrm{C}(2)-\mathrm{C}(3)$ & $-101.69(19)$ \\
\hline$C(11)-C(1)-C(2)-C(3)$ & $75.9(2)$ \\
\hline $\mathrm{O}(1)-\mathrm{C}(2)-\mathrm{C}(3)-\mathrm{C}(4)$ & $-10.5(2)$ \\
\hline$C(17)-C(2)-C(3)-C(4)$ & $-80.0(2)$ \\
\hline$C(1)-C(2)-C(3)-C(4)$ & $127.95(17)$ \\
\hline $\mathrm{O}(1)-\mathrm{C}(2)-\mathrm{C}(3)-\mathrm{C}(8)$ & $173.78(15)$ \\
\hline$C(17)-C(2)-C(3)-C(8)$ & $104.4(2)$ \\
\hline$C(1)-C(2)-C(3)-C(8)$ & $-47.7(2)$ \\
\hline$C(8)-C(3)-C(4)-C(5)$ & $4.0(3)$ \\
\hline$C(2)-C(3)-C(4)-C(5)$ & $-171.70(17)$ \\
\hline$C(3)-C(4)-C(5)-C(6)$ & $0.0(3)$ \\
\hline$C(4)-C(5)-C(6)-C(7)$ & $-3.5(3)$ \\
\hline $\mathrm{C}(4)-\mathrm{C}(5)-\mathrm{C}(6)-\mathrm{Cl}(1)$ & $174.91(14)$ \\
\hline$C(5)-C(6)-C(7)-C(8)$ & $2.8(3)$ \\
\hline $\mathrm{Cl}(1)-\mathrm{C}(6)-\mathrm{C}(7)-\mathrm{C}(8)$ & $-175.56(14)$ \\
\hline$C(6)-C(7)-C(8)-C(3)$ & $1.3(3)$ \\
\hline$C(6)-C(7)-C(8)-C(9)$ & $-178.10(17)$ \\
\hline$C(4)-C(3)-C(8)-C(7)$ & $-4.6(3)$ \\
\hline$C(2)-C(3)-C(8)-C(7)$ & $170.98(16)$ \\
\hline$C(4)-C(3)-C(8)-C(9)$ & $174.69(17)$ \\
\hline$C(2)-C(3)-C(8)-C(9)$ & $-9.7(3)$ \\
\hline$C(7)-C(8)-C(9)-C(10)$ & $179.70(17)$ \\
\hline$C(3)-C(8)-C(9)-C(10)$ & $0.4(3)$ \\
\hline$C(8)-C(9)-C(10)-C(11)$ & $62.5(2)$ \\
\hline$N(1)-C(1)-C(11)-C(12)$ & $-4.6(3)$ \\
\hline
\end{tabular}




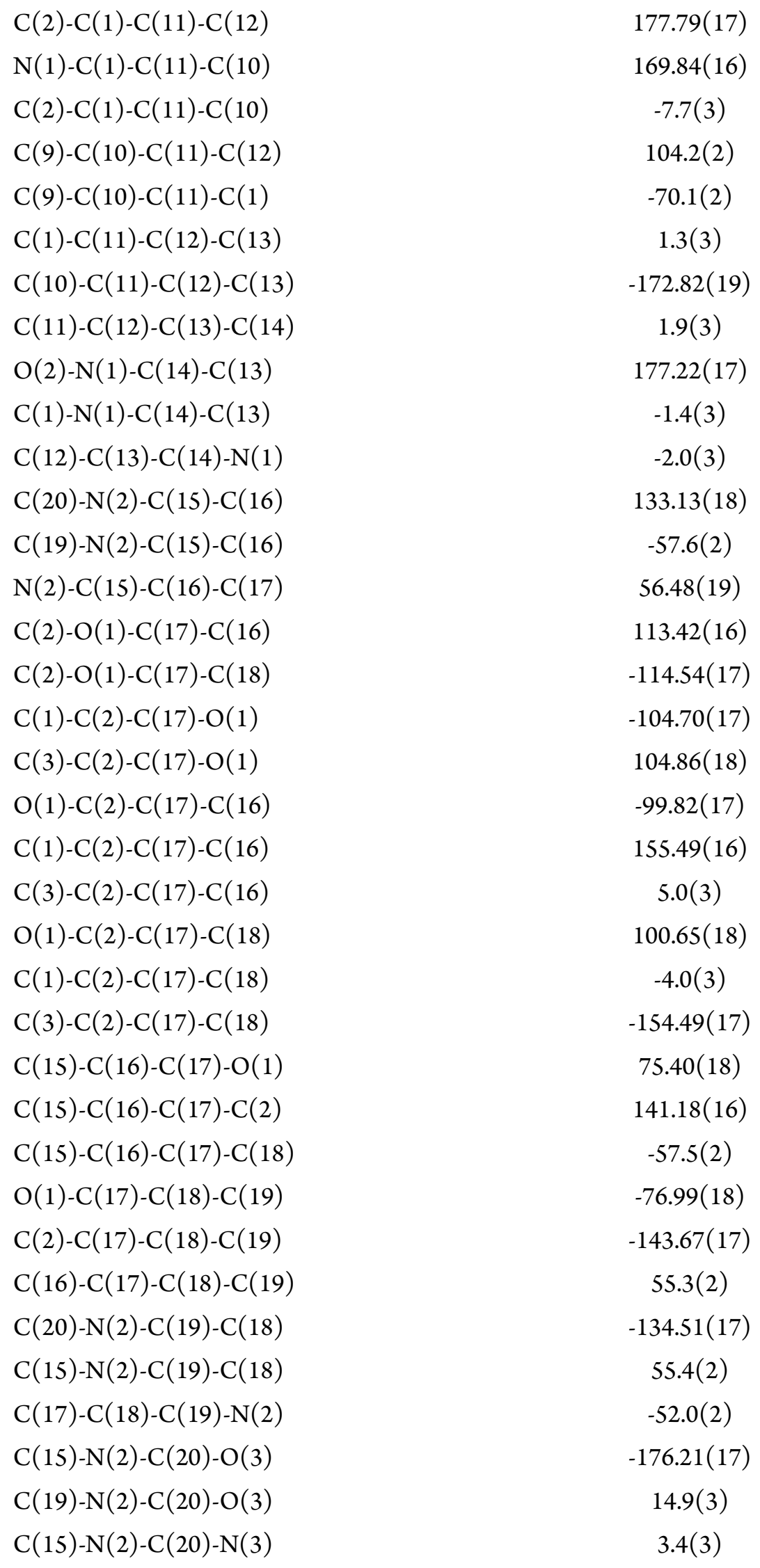




$\begin{array}{lc}\mathrm{C}(19)-\mathrm{N}(2)-\mathrm{C}(20)-\mathrm{N}(3) & -165.44(17) \\ \mathrm{C}(21)-\mathrm{N}(3)-\mathrm{C}(20)-\mathrm{O}(3) & 9.4(3) \\ \mathrm{C}(21)-\mathrm{N}(3)-\mathrm{C}(20)-\mathrm{N}(2) & -170.20(17) \\ \mathrm{C}(20)-\mathrm{N}(3)-\mathrm{C}(21)-\mathrm{C}(22) & 4.6(3) \\ \mathrm{C}(20)-\mathrm{N}(3)-\mathrm{C}(21)-\mathrm{C}(26) & -176.92(17) \\ \mathrm{C}(26)-\mathrm{C}(21)-\mathrm{C}(22)-\mathrm{C}(23) & -2.6(3) \\ \mathrm{N}(3)-\mathrm{C}(21)-\mathrm{C}(22)-\mathrm{C}(23) & 175.76(18) \\ \mathrm{C}(21)-\mathrm{C}(22)-\mathrm{C}(23)-\mathrm{C}(24) & 1.4(3) \\ \mathrm{C}(21)-\mathrm{C}(22)-\mathrm{C}(23)-\mathrm{C}(27) & 179.25(19) \\ \mathrm{C}(22)-\mathrm{C}(23)-\mathrm{C}(24)-\mathrm{C}(25) & 0.6(3) \\ \mathrm{C}(27)-\mathrm{C}(23)-\mathrm{C}(24)-\mathrm{C}(25) & -177.20(18) \\ \mathrm{C}(23)-\mathrm{C}(24)-\mathrm{C}(25)-\mathrm{C}(26) & -1.4(3) \\ \mathrm{C}(23)-\mathrm{C}(24)-\mathrm{C}(25)-\mathrm{C}(28) & 179.0(2) \\ \mathrm{C}(24)-\mathrm{C}(25)-\mathrm{C}(26)-\mathrm{C}(21) & 0.2(3) \\ \mathrm{C}(28)-\mathrm{C}(25)-\mathrm{C}(26)-\mathrm{C}(21) & 179.71(19) \\ \mathrm{C}(22)-\mathrm{C}(21)-\mathrm{C}(26)-\mathrm{C}(25) & 1.9(3) \\ \mathrm{N}(3)-\mathrm{C}(21)-\mathrm{C}(26)-\mathrm{C}(25) & -104.9(2) \\ \mathrm{C}(24)-\mathrm{C}(23)-\mathrm{C}(27)-\mathrm{F}(4) & 43.9(3) \\ \mathrm{C}(22)-\mathrm{C}(23)-\mathrm{C}(27)-\mathrm{F}(4) & -136.5(2) \\ \mathrm{C}(24)-\mathrm{C}(23)-\mathrm{C}(27)-\mathrm{F}(6) & -83.64(17) \\ \mathrm{C}(22)-\mathrm{C}(23)-\mathrm{C}(27)-\mathrm{F}(6) & -25.1(3) \\ \mathrm{C}(24)-\mathrm{C}(23)-\mathrm{C}(27)-\mathrm{F}(5) & 156.93(19) \\ \mathrm{C}(22)-\mathrm{C}(23)-\mathrm{C}(27)-\mathrm{F}(5) & -146.3(2) \\ \mathrm{C}(26)-\mathrm{C}(25)-\mathrm{C}(28)-\mathrm{F}(3) & 35.8(3) \\ \mathrm{C}(24)-\mathrm{C}(25)-\mathrm{C}(28)-\mathrm{F}(3) & -74.6(3) \\ \mathrm{C}(26)-\mathrm{C}(25)-\mathrm{C}(28)-\mathrm{F}(2) & \\ \mathrm{C}(24)-\mathrm{C}(25)-\mathrm{C}(28)-\mathrm{F}(2) & \\ \mathrm{C}(26)-\mathrm{C}(25)-\mathrm{C}(28)-\mathrm{F}(1) & \\ \mathrm{C}(24)-\mathrm{C}(25)-\mathrm{C}(28)-\mathrm{F}(1) & \\ & \\ & \\ & \end{array}$


Table S23. Hydrogen bonds for $8 \mathrm{~h}\left[\AA ̊\right.$ and $\left.{ }^{\circ}\right]$.

\begin{tabular}{lllll}
\hline D-H...A & $d(D-H)$ & $d(H \ldots A)$ & $d(D . . A)$ & $<($ DHA $)$ \\
\hline $\mathrm{O}(4)-\mathrm{H}(4 \mathrm{~A}) \ldots \mathrm{O}(3)$ & $0.84(3)$ & $1.90(3)$ & $2.742(2)$ & $178(3)$ \\
$\mathrm{O}(5)-\mathrm{H}(5 \mathrm{~A}) \ldots \mathrm{O}(2)$ & $0.96(4)$ & $1.91(4)$ & $2.859(2)$ & $173(4)$ \\
$\mathrm{O}(5)-\mathrm{H}(5 \mathrm{~A}) \ldots \mathrm{N}(1)$ & $0.96(4)$ & $2.63(4)$ & $3.490(2)$ & $149(3)$ \\
\hline
\end{tabular}




\section{Docking Studies with $6 \mathrm{a}$ and $6 \mathrm{~h}$ in $\mathrm{H}_{1} \mathrm{R}$}

The crystal structure of the human histamine receptor $\left(\mathrm{H}_{1} \mathrm{R}\right)$ bound to doxepin (PDB 3RZE, 3.1 Å resolution) $)^{12}$ was used as the protein in this study and was prepared with Schrödinger's PrepWizard utility. ${ }^{13}$ A 20 A grid of the protein alone was generated with rotatable hydroxyl groups on the residues closest to the center of the active site including: Tyr108, Ser111, Thr112, Thr194, Tyr431, Thr453, and Tyr458. Structures of ligands $6 \mathbf{a}$ and $\mathbf{6 h}$ in conformations a-d (see DFT section, S65) for both (+)- and (-)-enantiomers. These compounds were prepared in LigPrep ${ }^{14}$ using the OPLS3 force field ${ }^{15}$ and Epik, ${ }^{16}$ and then were docked with Glide SP. ${ }^{17}$ When ring sampling was enabled, only the (-)-enantiomer of each compound successfully docked in this binding pocket. Due to this biased result, we disabled ring sampling to ensure that the original conformation was docked. These compounds were also docked with Glide $\mathrm{XP}^{18}$ without ring sampling, yielding comparable results (Table S24). For each enantiomer of both compounds, the pose with the highest Glide SP score is shown in Figure 5.

Table S24. Comparison of Glide XP and SP scores for (+)- and (-)-6a and $6 \mathbf{h}$.

\begin{tabular}{cccr} 
Entry & Compound & Glide SP (kcal/mol) & Glide XP (kca/ $)$ \\
\hline 1 & $(+)-6 a$ & -9.180 & -8.650 \\
2 & $(-)-6 \mathbf{a}$ & -9.695 & -8.951 \\
3 & $(+)-6 \mathbf{h}$ & -10.317 & -9.766 \\
4 & $(-)-6 h$ & -10.218 & -9.817
\end{tabular}




\section{References}

1. Hsieh, S.-Y.; Tang, Y. U.; Crotti, S.; Stone, E. A.; Miller, S. J. Catalytic Enantioselective Pyridine $N$ Oxidation. J. Am. Chem. Soc. 2019, 141, 18624-18629.

2. Hansch, C.; Leo, A.; Taft, R. W. A survey of Hammett substituent constants and resonance and field parameters. Chem. Rev. 1991, 91, 165-195.

3. (a) Volpi, M.; Berlin, R. D. Intracellular elevations of free calcium induced by activation of histamine $\mathrm{H} 1$ receptors in interphase and mitotic HeLa cells: hormone signal transduction is altered during mitosis. J. Cell Biol. 1988, 107, 2533-2539; (b) Miller, T. R. Analysis of Apparent Noncompetitive Responses to Competitive $\mathrm{H}_{1}$-Histamine Receptor Antagonists in Fluorescent Imaging Plate Reader-Based Calcium Assays. J. Biomol. Screen. 1999, 4 (c) Mizuguchi, H.; Ono, S.; Hattori, M.; Sasaki, Y.; Fukui, H. Usefulness of HeLa cells to evaluate inverse agonistic activity of antihistamines. Int. Immunopharmacol. 2013, 15, 539-543.

4. M. J. Frisch, G. W. Trucks, H. B. Schlegel, G. E. Scuseria, M. A. Robb, J. R. Cheeseman, G. Scalmani, V. Barone, G. A. Petersson, H. Nakatsuji, X. Li, M. Caricato, A. V. Marenich, J. Bloino, B. G. Janesko, R. Gomperts, B. Mennucci, H. P. Hratchian, J. V. Ortiz, A. F. Izmaylov, J. L. Sonnenberg, D. Williams-Young, F. Ding, F. Lipparini, F. Egidi, J. Goings, B. Peng, A. Petrone, T. Henderson, D. Ranasinghe, V. G. Zakrzewski, J. Gao, N. Rega, G. Zheng, W. Liang, M. Hada, M. Ehara, K. Toyota, R. Fukuda, J. Hasegawa, M. Ishida, T. Nakajima, Y. Honda, O. Kitao, H. Nakai, T. Vreven, K. Throssell, J. A. Montgomery, Jr., J. E. Peralta, F. Ogliaro, M. J. Bearpark, J.J. Heyd, E. N. Brothers, K. N. Kudin, V. N. Staroverov, T. A. Keith, R. Kobayashi, J. Normand, K. Raghavachari, A. P. Rendell, J. C. Burant, S. S. Iyengar, J. Tomasi, M. Cossi, J. M. Millam, M. Klene, C. Adamo, R. Cammi, J. W. Ochterski, R. L. Martin, K. Morokuma, O. Farkas, J. B. Foresman, and D. J. Fox, Gaussian, Inc., Wallingford CT, 2016.

5. Stephens, P. J.; Devlin, F. J.; Chabalowski, C. F.; Frisch, M. J. Ab Initio Calculation of Vibrational Absorption and Circular Dichroism Spectra Using Density Functional Force Fields. J. Phys. Chem. 1994, 98, $11623-11627$.

6. Becke, A. D. Density-functional thermochemistry. III. The role of exact exchange. J. Chem. Phys. 1993, 98, 5648-5652.

7. Lee, C.; Yang, W.; Parr, R. G. Development of the Colle-Salvetti correlation-energy formula into a functional of the electron density. Phys. Rev. B 1988, 37, 785-789.

8. Krishnan, R.; Binkley, J. S.; Seeger, R.; Pople, J. A. Self-consistent molecular orbital methods. XX. A basis set for correlated wave functions. J. Chem. Phys. 1980, 72, 650-654.

9. Grimme, S.; Antony, J.; Ehrlich, S.; Krieg, H. A consistent and accurate ab initio parametrization of density functional dispersion correction (DFT-D) for the 94 elements H-Pu. J. Chem. Phys. 2010, 132, 154104.

10. CrysAlisPro; Rigaku OD: The Woodlands, TX, 2015.

11. Sheldrick, G. M. A short history of SHELX. Acta Cryst. 2008, A64, 112-122.

12. Shimamura, T.; Shiroishi, M.; Weyand, S.; Tsujimoto, H.; Winter, G.; Katritch, V.; Abagyan, R.; Cherezov, V.; Liu, W.; Han, G. W.; Kobayashi, T.; Stevens, R. C.; Iwata, S., Structure of the Human Histamine $\mathrm{H}_{1}$ Receptor Complex with Doxepin. Nature 2011, 475, 65-70.

13. (a) Madhavi Sastry, G.; Adzhigirey, M.; Day, T.; Annabhimoju, R.; Sherman, W., Protein and Ligand Preparation: Parameters, Protocols, and Influence on Virtual Screening Enrichments. J. Comp. Aided Mol. Des. 2013, 27, 221-234. (b) Schrödinger Release 2015-4: Protein Preparation Wizard; Epik, Schrödinger, LLC, New York, NY, 2015; Impact, Schrödinger, LLC, New York, NY, 2015; Prime, Schrödinger, LLC, New York, NY, 2015.

14. Schrödinger Release 2015-4: LigPrep, Schrödinger, LLC, New York, NY, 2015.

15. (a) Jorgensen, W. L.; Tirado-Rives, J., The OPLS [Optimized Potentials for Liquid Simulations] Potential Functions for Proteins, Energy Minimizations for Crystals of Cyclic Peptides and Crambin. J. Am. Chem. Soc. 1988, 110, 1657-1666. (b) Jorgensen, W. L.; Maxwell, D. S.; Tirado-Rives, J., Development and 
Testing of the OPLS All-Atom Force Field on Conformational Energetics and Properties of Organic Liquids. J. Am. Chem.Soc. 1996, 118, 11225-11236. (c) Shivakumar, D.; Williams, J.; Wu, Y.; Damm, W.; Shelley, J.; Sherman, W., Prediction of Absolute Solvation Free Energies using Molecular Dynamics Free Energy Perturbation and the OPLS Force Field. J. Chem. Theory Comput. 2010, 6, 1509-1519. (d) Harder, E.; Damm, W.; Maple, J.; Wu, C.; Reboul, M.; Xiang, J. Y.; Wang, L.; Lupyan, D.; Dahlgren, M. K.; Knight, J. L.; Kaus, J. W.; Cerutti, D. S.; Krilov, G.; Jorgensen, W. L.; Abel, R.; Friesner, R. A., OPLS3: A Force Field Providing Broad Coverage of Drug-like Small Molecules and Proteins. J. Chem. Theory Comput. 2016, 12, 281-296.

16. (a) Shelley, J. C.; Cholleti, A.; Frye, L. L.; Greenwood, J. R.; Timlin, M. R.; Uchimaya, M., Epik: a software program for pKaprediction and protonation state generation for drug-like molecules. J. Comput. Aided Mol. Des. 2007, 21, 681-691. (b) Greenwood, J. R.; Calkins, D.; Sullivan, A. P.; Shelley, J. C., Towards the comprehensive, rapid, and accurate prediction of the favorable tautomeric states of drug-like molecules in aqueous solution. J. Comput. Aided Mol. Des. 2010, 24, 591-604. (c) Schrödinger Release 2015-4: Epik, Schrödinger, LLC, New York, NY, 2015.

17. (a) Friesner, R. A.; Banks, J. L.; Murphy, R. B.; Halgren, T. A.; Klicic, J. J.; Mainz, D. T.; Repasky, M. P.; Knoll, E. H.; Shelley, M.; Perry, J. K.; Shaw, D. E.; Francis, P.; Shenkin, P. S., Glide: A New Approach for Rapid, Accurate Docking and Scoring. 1. Method and Assessment of Docking Accuracy. J. Med. Chem. 2004, 47, 1739-1749. (b) Halgren, T. A.; Murphy, R. B.; Friesner, R. A.; Beard, H. S.; Frye, L. L.; Pollard, W. T.; Banks, J. L., Glide: A New Approach for Rapid, Accurate Docking and Scoring. 2. Enrichment Factors in Database Screening. J. Med. Chem. 2004, 47, 1750-1759.

18. Friesner, R. A.; Murphy, R. B.; Repasky, M. P.; Frye, L. L.; Greenwood, J. R.; Halgren, T. A.; Sanschagrin, P. C.; Mainz, D. T., Extra Precision Glide: Docking and Scoring Incorporating a Model of Hydrophobic Enclosure for Protein-Ligand Complexes. J. Med. Chem. 2006, 49, 6177-6196. 